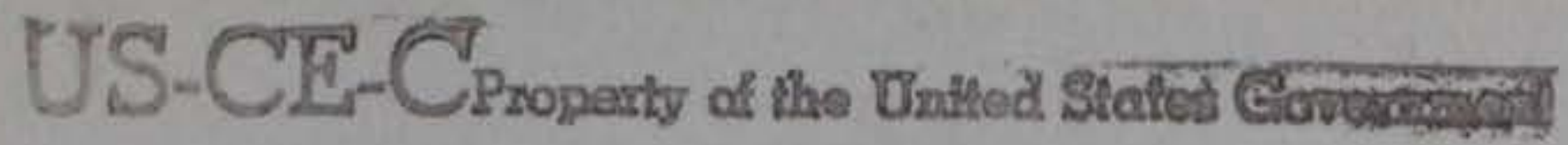
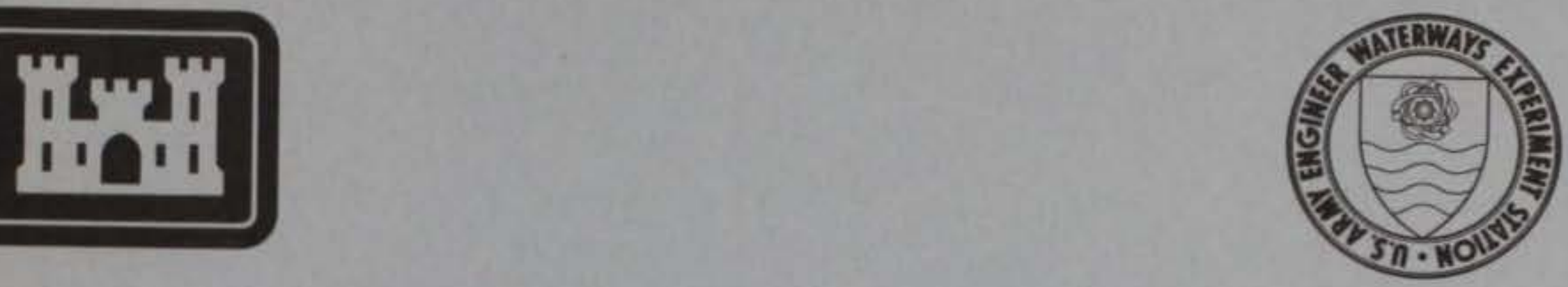

TECHNICAL REPORT GL-82-4

\section{STEERABILITY ANALYSIS OF TRACKED VEHICLES ON SOFT SOIL; THEORETICAL PREDICTIONS VERSUS FIELD MEASUREMENTS}

by

Behzad Rohani and George Y. Baladi

Geotechnical Laboratory

U. S. Army Engineer Waterways Experiment Station

P. O. Box 631, Vicksburg, Miss. 39180

June 1982

Final Report

Approved For Public Release; Distribution Unlimited

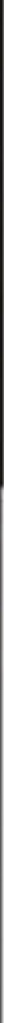

Prepared for Office, Chief of Engineers, U. S. Army

Washington, D. C. 20314

Under Project 4A161102AT22, Task CO, Work Unit 001

Monitored by Geotechnical Laboratory

U. S. Army Engineer Waterways Experiment Station

P. O. Box 63 Lubtioks sargectMiss. 39180

TECHNICAL INFORMATION CENTER

US ARMAY ENGINEER WATERWAYS EXPERIMENT STATION 


\begin{tabular}{|c|c|}
\hline REPORT DOCUMENTATION PAGE & $\begin{array}{l}\text { READ INSTRUCTIONS } \\
\text { BEFORE COMPLETING FORM }\end{array}$ \\
\hline $\begin{array}{l}\text { 1. REPORT NUMBER } \\
\text { Technical Report GL-82-4 }\end{array}$ & 3. RECIPIENT'S CATALOG NUMBER \\
\hline $\begin{array}{l}\text { 4. TITLE (and Subtito) } \\
\text { STEERABILITY ANALYSIS OF TRACKED VEHICLES ON SOFT } \\
\text { SOIL; THEORETICAL PREDICTIONS VERSUS FIELD } \\
\text { MEASUREMENTS }\end{array}$ & $\begin{array}{l}\text { 5. TYPE OF REPORT A PERIOD COVERED } \\
\text { Final rEPOIT } \\
\text { 5. PERFORMING ORG. REPORT NUMBER }\end{array}$ \\
\hline $\begin{array}{l}\text { 7. AUTHOR(*) } \\
\text { Behzad Rohani } \\
\text { George Y. Baladi }\end{array}$ & 8. CONTRACT OR GRANT NUMBER(D) \\
\hline $\begin{array}{l}\text { 9. PERFORMING ORGANIZATION NAME AND ADDRESS } \\
\text { U. S. Army Engineer Waterways Experiment Station } \\
\text { Structures Laboratory } \\
\text { P. O. Box } 631 \text {, Vicksburg, Miss. } 39180\end{array}$ & $\begin{array}{l}\text { 10. PROGRAM ELEMENT, PROJECT, TASK } \\
\text { AREA Q WORK UNIT' NUMBERS } \\
\text { Project 4A161102AT22, } \\
\text { Task co, Work Unit } 001\end{array}$ \\
\hline $\begin{array}{l}\text { 11. CONTROLLING OFFICE NAME AND ADDRESS } \\
\text { Office, Chief of Engineers, U. S. Army } \\
\text { Washington, D. C. } 20314\end{array}$ & $\begin{array}{l}\text { 12. REPORT DATE } \\
\text { June } 1982 \\
\text { 13. NUMBER OF PAGES } \\
214\end{array}$ \\
\hline $\begin{array}{l}\text { 14. MONITORING AGENCY NAME A ADDRESS(II difforent from Controlling Oflice) } \\
\text { Geotechnical Laboratory } \\
\text { U. S. Army Engineer Waterways Experiment Station }\end{array}$ & $\begin{array}{l}\text { 15. SECURITY CLASS. (of thio roport) } \\
\text { Unclassified }\end{array}$ \\
\hline P. 0. Box 631 , Vicksburg, Miss. 39180 & $\begin{array}{l}\text { 15a. DECLASSIFICATION/DOWNGRADING } \\
\text { SCHEDULE }\end{array}$ \\
\hline
\end{tabular}

16. DISTRIBUTION STATEMENT (Ol thlo Roport)

Approved for public release; distribution unlimited.

17. DISTRIBUTION STATEMENT (of the abatract ontered in Block 20, 11 difforent from Report)

18. SUPPLEMENTARY NOTES

Available from National Technical Information Service, 5285 Port Royal Road, Springfield, Va. 22151.

19. KEY WORDS (Continue on reverse side If necessary and identify by block number)

AGIL (Computer program)

Mathematical models

Military vehicles

Rheological models
Soil-track interaction

Terrain-vehicle interaction

Track-laying vehicles

Vehicle performance

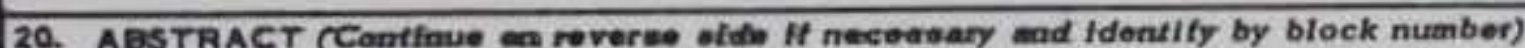

In 1978, a terrain-vehicle interaction model was developed by the authors for predicting the steering performance of high-mobility/agility tracked vehicles in environments ranging from very soft soils to hard surfaces. To validate the accuracy of the model for predicting the steering performance of tracked vehicles on soft soils, circular-turn tests were conducted at two sites on a floodplain north of Redwood, Mississippi, and on a dredged spoil area

(Continued) 


\section{ABSTRACT (Continued).}

within the WES reservation at Vicksburg, Mississippi. A number of in situ direct shear tests were conducted at each test site and the results used to fit a mathematical soil model with a rate-dependent nonlinear failure envelope for input to the tracked vehicle prediction model. Thirty-five circular-turn tests were conducted at the three test sites, but due to instrumentation problems, complete data were collected for only 16 tests at the Redwood sites. Results from these 16 "good tests" were used to validate both the steady-state and the transient versions of the model.

For the transient predictions, Fourier series were fit to the entire measured time histories of the inner and outer track velocities and used as input to the model. Calculated time histories of vehicle speed, lateral acceleration, power required at the sprockets, and the trajectory of the center of gravity of the vehicle were compared with corresponding test measurements. For the steadystate predictions, five tests were selected and the mean measured radius for each test was used as input to the model. The model output consisted of a maximum vehicle velocity based on either specified stability criteria or power available at the sprockets. Predictions for both transient and steady-state conditions compared very favorably with the test data, indicating that the model is capable of predicting the steering performance of tracked vehicles on soft soil. 
This investigation was conducted for the office, Chief of Engineers, U. S. Army, by personnel of the U. S. Army Engineer Waterways Experiment Station (WES), CE, as a part of Project 4Al61102AT22, Task C0, Work Unit 001, "Dynamic Soil-Track Interactions Governing High-Speed Tracked Vehicle Performance."

The mathematical model, prediction methodology, and analyses reported herein were performed by Drs. Behzad Rohani and George Y. Baladi of the Geomechanics Division (GD), Structures Laboratory, during the period October 1980 - October 1981 under the general direction of Mr. C. J. Nuttall, Jr., Chief, Mobility Systems Division (MSD), Geotechnical Laboratory (GL), and Dr. W. F. Marcuson III, Chief, GL. The field test program was directed by Messrs. N. R. Murphy, Jr., B. G. Schreiner, and C. E. Green, MSD. The field direct shear device described in Appendix B for measurements of soil properties was designed by Mr. J. Q. Ehrgott, GD. The field measurements of the vehicle performance were processed by Mr. P. J. Kuykenda11, MSD. Numerical calculations using the WES terrain-vehicle interaction model were performed by Mr. D. E. Barnes and Mrs. J. T. Carlisle, GD. This report was written by Drs. Rohani and Baladi.

COL Nelson P. Conover, CE, and COL Tilford C. Creel, CE, were Commanders and Directors of the WES during the investigation. Mr. Fred R. Brown was Technical Director. 
CONVERSION FACTORS, U. S. CUSTOMARY TO METRIC (SI)

UNITS OF MEASUREMENT . . . . . . . . . . . . . . . . 3

PART I: INTRODUCTION . . . . . . . . . . . . . . 4

Background ................... . . . . 4 4

Purpose and Scope................... . . . 5

PART II: SITE CHARACTERIZATION . . . . . . . . . . . 6

Background .................... . . . . . 6

Test Sites .................... . . . . 6

Direct Shear Test Results . . . . . . . . . . . 7

Material Constants for Soil Model . . . . . . . . . 8

PART III: COMPARISON OF THEORETICAL PREDICTIONS WITH

TEST RESULTS . . . . . . . . . . . . . . . 11

Test Procedure . . . . . . . . . . . . . . . . 11

Prediction Methodology .. . . . . . . . . . . 12

Theoretical Predictions . . . . . . . . . . . . . 13

PART IV: SUMMARY AND RECOMMENDATIONS . . . . . . . . . . . 18

REFERENCES . . . . . . . . . . . . . . . . . . 20

TABLES $1-13$

FIGURES $1-112$

APPENDIX A: TERRAIN-VEHICLE INTERACTION MODEL . . . . . . . . AI

Introduction . . . . . . . . . . . . . . . . . . Al

Soil Model . . . . . . . . . . . . . . . . . $\mathrm{A}^{\mathrm{A}}$

Derivation of Terrain-Vehicle Model . . . . . . . . . A4

FIGURES Al-A11

APPENDIX B: DESCRIPTION OF THE FIELD DIRECT SHEAR DEVICE..$\quad$ B1

Background ................. B1

Direct Shear Device............... B2

Measurement of Soil Parameters . . . . . . . . . . B5

Conclusions .................. . B6

FIGURES B1-B5

APPENDIX C: SOIL ClASSIFICATION TEST DATA . . . . . . . . $\mathrm{Cl}$ FIGURES $\mathrm{C} 1-\mathrm{C} 24$

APPENDIX D: NOTATION . . . . . . . . . . . . . . D1 
U. S. customary units of measurement used in this report can be converted to metric (SI) units as follows:

\begin{tabular}{|c|c|c|}
\hline Multiply & By & To Obtain \\
\hline feet & 0.3048 & metres \\
\hline horsepower & 745.6999 & watts \\
\hline inches & 25.4 & millimetres \\
\hline inches per second & 25.4 & millimetres per second \\
\hline miles per hour (U. S. statute) & 1.609344 & kilometres per hour \\
\hline pounds (force) & 4.448222 & newtons \\
\hline $\begin{array}{l}\text { pounds (force)-inch-second } \\
\text { squared }\end{array}$ & 0.11306064 & kilograms-square metres \\
\hline pounds (force) per cubic inch & 0.2714 & megapascals per metre \\
\hline pounds (force) per square inch & 6.894757 & kilopascals \\
\hline pounds (mass) & 0.4535924 & kilograms \\
\hline pounds (mass) per cubic foot & 16.01846 & kilograms per cubic metre \\
\hline square inches & 6.4516 & square centimetres \\
\hline
\end{tabular}


STEERABILITY ANALYSIS OF TRACKED VEHICLES ON SOFT SOIL; THEORETICAL PREDICTIONS VERSUS FIELD MEASUREMENTS

\section{PART I: INTRODUCTION}

\section{Background}

1. Development of high-mobility/agility tracked combat vehicles has received considerable attention recently because of the possibilities these vehicles offer for increased battlefield survivability through the avoidance, by high-speed and violent maneuver, of hits by high-velocity projectiles and missiles. In order to design and develop such vehicles rationally, it is necessary to have a quantitative understanding of the interrelationship between the terrain factors (soil type, soil shear strength, and compressibility, etc.) and the vehicle characteristics (weight, track length and width, location of center of gravity, etc.) during steering. The actual mechanism of terrainvehicle interaction during steering is undoubtedly very complex. Thus, in order to study such an interrelationship, it is necessary to construct idealized mathematical models of the actual system. The accuracy and range of application of such models must, of course, be determined from actual mobility experiments and obviously must depend on the degree of relevance of the idealized model as an approximation to the real behavior. A research program was initiated at the U. S. Army Engineer Waterways Experiment Station (WES) in 1976 to develop a mathematical model of terrain-vehicle interaction for predicting the steering performance of ground-crawling vehicles operating off the road. Deve1opment of the model was completed in 1978 (Baladi and Rohani, 1979). For its initial validation, results from only five circular-turn tests, all conducted at one site, were available for comparison with theoretical predictions (Baladi and Rohani, 1981). Data from 35 tests conducted at three different soft-soil sites have recently been reduced and are 
now available for further investigation of the model's validity (Schreiner and Green, 1980; Green (in preparation)).

\section{Purpose and Scope}

2. The purpose of this report is to assess the ability of the WES terrain-vehicle interaction model to predict the steering performance of tracked vehicles on soft soils by comparing test results with model predictions. The test data are limited to the steering performance of a selected track-laying vehicle tested at three different soft-soil sites. The characteristics of the soil at the test locations are described and values of the soil model material constants are developed from in situ direct shear measurements in Part II. The test procedure, prediction methodology, and comparisons of model predictions with test data are presented in Part III. Part IV contains a summary and recommendations for future work. In Appendix A the terrain-vehicle model reported by Baladi and Rohani (1979 and 1981) is extended to include the treatment of sloping terrain under nonuniform (transient) turning motion. Description and use of a direct shear device for measurement of pertinent soil properties are documented in Appendix B. Soil classification data for all test sites are given in Appendix C. 


\section{PART II: SITE CHARACTERIZATION}

\section{Background}

3. One of the most important engineering properties of a soil affecting trafficability is its in situ shear strength. The shear strength of earth materials varies greatly for different types of soil and is dependent on the confining pressure and time rate of loading (shearing). This dependence, however, varies with respect to the soil's cohesive and frictional properties. It has been found experimentally that the shear strength of purely cohesive soils (such as a saturated plastic clay) is relatively independent of the confining stress, but strongly affected by the time rate of shearing. On the other hand, the shear strength of purely frictional soils (such as a dry clean sand) is found to be relatively independent of time rate of loading, but strongly dependent on confining pressure. The shearing resistance of most soils, however, is due to both frictional and cohesive components. An appropriate test for determining shear strength for application in mobility studies is a direct shear test conducted in situ on the soil surface. A field direct shear device has been developed at the WES for such applications and is documented in Appendix B. This device was used to measure the in situ shear strength of the soil at each test location.

\section{Test Sites}

4. The mobility tests were conducted at two sites (test sites 7A and $7 \mathrm{~B}$ ) on a floodplain north of Redwood, Mississippi, and on a hydraulically-filled dredge spoil area (test site 8) within the WES reservation at Vicksburg, Mississippi (Schreiner and Green, 1980). The floodplain soil at Redwood is a soft, plastic clay classified as $\mathrm{CH}$ 
according to the Unified Soil Classification System* (USCS); however, test site 7A was on plowed ground while test site 7B was on natural ground. The dredged soil at test site 8 is a lean, brown clay classified as CL according to the USCS. Six test series were conducted at these sites during June 1979. Table 1 lists the location and date of each test series and also includes the average rating cone index (RCI), a measure of the soil's remolded resistance to penetration by the standard WES cone penetrometer (Smith, 1964), for each test series. Soil classification data for the test locations are given in Appendix $\mathrm{C}$. It is noted from Table 1 that within each test site the strength of the soil varies for different test locations. For example, test series 107111 and 139-144 were both conducted at test site $7 \mathrm{~B}$, but the strength of the soil at the two test locations is different.

\section{Direct Shear Test Results}

\section{Eighty-three direct shear tests were conducted at the test} sites using the procedure outlined in Appendix B.** Both slow and fast tests were conducted to ascertain the sensitivity of the shear strength of the material to the rate of deformation. In the case of fast tests, the soil specimens were sheared at a strain rate of approximately 0.125 to 0.25 per sec. This range of strain rates is compatible with the average strain rate experienced by the soil particles under the track of the test vehicle during steering. This strain rate is estimated from slip velocity calculations to be on the order of 0.1 per sec. The slip velocity calculations are based on actual measurements of track velocity and vehicle speed during steering.

* The Unified Soil Classification System is described in Technical Memorandum No. 3-357 by the U. S. Army Engineer Waterways Experiment Station (1953).

** Raw data from these tests are available at the WES. Only a summary of the synthesized data necessary for model predictions is presented in this report. 
6. Direct shear tests were conducted using applied normal loads of $8.6,36.6,65.7$, and $122.71 \mathrm{~b} *$ corresponding, respectively, to normal stresses of $0.54,2.29,4.11$, and $7.67 \mathrm{psi}$. The ground contact pressure for the test vehicle is $5.71 \mathrm{psi}$. Therefore, the range of normal stresses used in the test program is applicable to the test vehicle of interest. Figure 1 shows typical load-deformation data obtained from the direct shear device. As indicated in Figure 1 in the case of fast tests, the soil specimens were monotonically sheared to failure, whereas the slow tests experienced several load-unload cycles of deformation. The load-deformation data were used to construct representative failure curves for the six test locations indicated in Table 1. The representative failure curves are portrayed in Figures 2-7 as plots of peak shear load versus normal load for both the slow and fast tests. For load-deformation curves that did not exhibit a distinct peak, the value of shear load at 15 percent strain (corresponding to $0.6 \mathrm{in.}$ of deformation) was selected to construct the failure curves. The data indicate that the increase in the shear load beyond 15 percent strain is negligible. The average values of wet density and water content given for each of the test locations in Figures 2-7 correspond to the average of the wet density and water content of the soil specimens tested at each location. The failure curves in these figures clearly demonstrate that the shear strength of the soil for all three sites is sensitive to the rate of deformation. It is further observed from Figures 2-7 that the shear strength of the CL soil at site 8 is considerably higher than the shear strength of the $\mathrm{CH}$ soil at sites 7A and 7B. The same relative difference in shear strength between the two materials is also reflected in the RCI readings listed in Table 1.

\section{Material Constants for Soil Model}

7. As pointed out previously, the purpose of the direct shear

* A table of factors for converting U. S. customary units of measurements to metric (SI) units is presented on page 3 . 
tests was to determine the in situ shear strength of the material for site characterization within the framework of the soil model described in Appendix A. Such a characterization is necessary in order to use the WES terrain-vehicle interaction model for predicting the steering performance of the vehicle on the particular terrain of interest. The soil model contains six material constants. Three of the material constants ( $A, M$, and $N$ ) describe the static failure envelope of the material (see Equation Al and Figure Al, Appendix A); two parameters ( $\Lambda$ and $C_{d}$ ) define the contribution to cohesive strength of the material due to dynamic loading (see Equation A2 and Figure A1); and one parametẹ (G) defines the initial shear stiffness coefficient of the soil (see Equation $\mathrm{A} 3$ and Figure A2). The first step in determining the numerical values of the material constants $A, M$, and $N$ is to convert the slow test failure curves in Figures $2-7$ to shear strength $\tau_{M}$ versus normal stress $\sigma$ relations by simply dividing the shear load and normal load by the cross-sectional area of the soil specimen (4- by 4-in. specimen). The second step involves fitting Equation Al to the resulting $\tau_{M}$ versus $\sigma$ curves. Figures $8-13$ portray the experimental $\tau_{M}$ versus $\sigma$ curves and the corresponding model behavior for each of the test locations. It is observed from these figures that the agreement between field measurements and model behavior is excellent. The parameter $\mathrm{C}_{\mathrm{d}}$, corresponding to the increase in soil cohesion due to dynamic loading (maximum loading rate of interest), can be determined from Figures 2-7. Basically, $C_{d}$ corresponds to the difference between the fast and slow failure curves at zero normal load divided by the cross-sectional area of the specimen. The parameter $\Lambda$ defines the rate of increase in soil cohesion due to deformation velocity (Equation A2). If shear test data were available for several rates of deformation, the value of $\Lambda$ would be determined by fitting Equation A2 (for $\sigma=0$ ) to a plot of cohesion versus deformation velocity, as indicated in Figure B5, Appendix B. In the absence of such information, however, $\Lambda$ is treated as a fitting parameter; i.e., the value of $\Lambda$ is determined on the basis of fitting Equation A4 to the stress-deformation 
curves from fast tests. Before fitting Equation A4 to the stressdeformation curves, the value of the initial shear stiffness coefficient $\mathrm{G}$ must be known. The parameter $\mathrm{G}$ is, in general, dependent on the normal stress and the deformation velocity (Figure B5). Within the framework of the present soil model, however, G is assumed to be a constant and independent of either the normal stress or the deformation velocity. Parametric studies conducted with the terrain-vehicle model have indicated that the steering performance of the vehicle is only mildly dependent on the soil parameter G. Therefore, it is not necessary to characterize this parameter precisely. The procedure adopted to determine $\mathrm{G}$ is first to plot the initial slopes of the stress-deformation curves versus normal stress from both the slow and fast tests. Then, using this plot, select an average value of $G$ at a normal stress level corresponding to the ground contact pressure for the vehicle of interest.

8. The numerical values of the six material constants were determined for all the test locations following the above procedures and are summarized in Table 2. To demonstrate the validity of the soil model for simulating the stress-deformation response of the material from direct shear tests, typical experimental data from fast tests are compared in Figures 14-19 with the corresponding model behavior. The deformation velocity $\dot{\Delta}$ of $0.75 \mathrm{in.} / \mathrm{sec}$ used in the soil model calculations corresponds to an average velocity for the fast tests. The comparisons were made for a normal stress $\sigma$ of 4.11 psi, the direct shear test value that was closest to the ground contact pressure of the test vehicle $(5.71 \mathrm{psi})$. Comparisons of the field data with model behavior in Figures 14-19 indicate that the soil model is capable of simulating the pertinent features of the shear stress-deformation response of the material very accurately. Some of the field measurements (e.g., Figures 18 and 19) exhibit some degree of strain-softening that cannot be simulated with the current version of the soil model. However, for the present application of the model, such strain-softening behavior is not a significant phenomenon. 
PART III: COMPARISON OF THEORETICAL PREDICTIONS WITH TEST RESULTS

\section{Test Procedure}

9. The field tests consisted of a number of circular-turn tests; details of the test procedure are documented in a study by Green (in preparation). The principal objectives of the circular-turn tests were: (a) determine the effects of turning radius on turning performance in terms of vehicle speed and power requirements, (b) determine the effects of soil strength on turning performance, and (c) develop a dața base to check the accuracy and range of application of the WES terrain-vehicle interaction model. Basically, each test involved running the tracked vehicle in a circular path by first accelerating the vehicle to a maximum speed (controlled by either the available power or the actual physical stability of the vehicle) and then continuously turning it in a near steady-state condition. The tracked vehicle used for the field tests is an armored personnel vehicle with the characteristics listed in Table 3. The actual data collected during each test consisted of time histories of (a) sprocket rpm and torque, (b) turning radius, and (c) velocity of the vehicle. Then the track velocities, lateral acceleration, and power consumption were calculated for each test using these measurements and appropriate equations (Figure 20). In addition to the above data, stopwatch times for each revolution and posttest measurements from the center of the circle to the inner and outer track ruts were obtained to calculate an average effective vehicle speed and turning radius for each circular path. This information provided a check on the turning instrumentation. Also, in order to determine the coefficient of rolling resistance 6 (see Equation A46), acceleration/deceleration (AC/DC) tests were conducted at each test location (Green (in preparation)). Table 4 lists the resulting values of 6 for the six test locations. 


\section{Prediction Methodology}

10. The prediction methodology associated with the WES terrainvehicle interaction model is illustrated in Figure 21 . The model input consists of three separate sets of data. The first set of data describes the mechanical properties of the terrain within the framework of the soil model described in Appendix A. Table 2 presents the data necessary for this set of input parameters for all test locations. The second set of data describes the characteristics of the vehicle and the coefficient of rolling resistance for each test location. Tables 3 and 4 outline such information for the vehicle of interest for all the test locations. The third set of input data describes the conditions by which one would drive the model. There are several combinations of driving conditions that can be used depending on the nature of the particular problem at hand (Baladi and Rohani, 1979). For the particular application of interest in this report, the driving conditions consist of the time histories of the inner and outer track velocities, which are obtained from actual field measurements. For these specified driving conditions, the model outputs the time histories of vehicle velocity, slip velocities, lateral acceleration, power at sprockets, turning radius, and offset. These quantities can then be compared with corresponding field data in order to determine the accuracy of the model. As indicated in Figure 20, the field data for the circular-turn tests consist of vehicle velocity, turning radius, lateral acceleration, and power at the sprockets. The average slip velocity can also be calculated from the direct field measurements if desired.

11. In using the time histories of track velocities to drive the terrain-vehicle interaction model, the field measurements were filtered and fitted by analytical expressions because the field data included artificial high-frequency oscillations that are believed to be instrumentation "noise." These oscillations lead to frequent crossings of the two track velocity-time histories, which are unrealistic for the circular-turn tests of interest. To filter the data, each track velocity-time history was first numerically integrated. The integrated 
results were plotted versus time and were represented by a Fourier series containing 21 terms. The Fourier series was then differentiated to yield an expression for the track velocity. The differentiated analytical expression for the track velocity has the following form

$$
v_{x}=A_{1}+B_{1}+\sum_{i=2}^{21} \frac{2 \pi(i-1)}{T_{t}}\left\{B_{i} \cos \left[\frac{2 \pi(i-1) t}{T_{t}}\right]-A_{i} \sin \left[\frac{2 \pi(i-1) t}{T_{t}}\right]\right\}
$$

where

$$
\begin{aligned}
& v_{x}=\text { track velocity } \\
& T_{t}=\text { total time indicating the duration of the event } \\
& t=\text { time } \\
& A_{i}, B_{i}(i=1, \ldots, 21)=\text { constants }
\end{aligned}
$$

The numerical values of the coefficients $A_{i}$ and $B_{i}$ are given in Tables 5-13 for use of Equation 1 by interested individuals. Comparisons of the filtered data and field measurements for all the tests are presented in the next section.

\section{Theoretical Predictions}

12. The experimental program consisted of 35 circular tests divided into six test series (Table 1). Unfortunately, due to instrumentation problems, complete data (as described in Figure 20) were collected for only 16 tests. These tests are referred to as "good tests." The data for other tests were either partially complete or of poor quality. The theoretical predictions reported in this section include the 16 good tests (all from sites $7 \mathrm{~A}$ and $7 \mathrm{~B}$ ) and one test with poor quality data (from site 8). The 16 tests consist of tests 99, 100, $101,102,107,108,109,110,111,117,118,133,134,139,140$, and 141. Test 157 (from test series 157-159) was selected as an example of a test with poor quality data. Two types of predictions are made for the tests above and are discussed in the following paragraphs. 
Complete transient simulation

13. To demonstrate the ability of the terrain-vehicle model for predicting transient motion, the response of the vehicle was simulated for the entire test event. The results of these simulations are portrayed in five figures for each test. The first figure consists of the time histories of the outer and inner track velocities that constitute the driving conditions for the model. This figure includes both the field measurements and the filtered data (see paragraph 11), which are used as input to the model. The next three figures show the time histories of the vehicle speed, lateral acceleration, and power requirement, and include both the field measurements and the corresponding model predictions. The fifth figure shows the trajectory of the center of gravity of the vehicle and includes both field measurements and predictions. Figures 22-106 show the simulation results for the 17 tests indicated in paragraph 12 . The figure numbers corresponding to each test are listed in the following tabulation:

Figure No.

\begin{tabular}{|c|c|c|c|c|c|}
\hline $\begin{array}{l}\text { Test } \\
\text { No. }\end{array}$ & $\begin{array}{l}\text { Track } \\
\text { Velocity }\end{array}$ & $\begin{array}{l}\text { Vehicle } \\
\text { Velocity }\end{array}$ & $\begin{array}{c}\text { Lateral } \\
\text { Acceleration } \\
\end{array}$ & $\begin{array}{c}\text { Power } \\
\text { Requirement } \\
\end{array}$ & Trajectory \\
\hline 99 & 22 & 23 & 24 & 25 & 26 \\
\hline 100 & 27 & 28 & 29 & 30 & 31 \\
\hline 101 & 32 & 33 & 34 & 35 & 36 \\
\hline 102 & 37 & 38 & 39 & 40 & 41 \\
\hline 107 & 42 & 43 & 44 & 45 & 46 \\
\hline 108 & 47 & 48 & 49 & 50 & 51 \\
\hline 109 & 52 & 53 & 54 & 55 & 56 \\
\hline 110 & 57 & 58 & 59 & 60 & 61 \\
\hline 111 & 62 & 63 & 64 & 65 & 66 \\
\hline 117 & 67 & 68 & 69 & 70 & 71 \\
\hline 118 & 72 & 73 & 74 & 75 & 76 \\
\hline 133 & 77 & 78 & 79 & 80 & 81 \\
\hline 134 & 82 & 83 & 84 & 85 & 86 \\
\hline 139 & 87 & 88 & 89 & 90 & 91 \\
\hline 140 & 92 & 93 & 94 & 95 & 96 \\
\hline 141 & 97 & 98 & 99 & 100 & 101 \\
\hline $157 *$ & 102 & 103 & 104 & 105 & 106 \\
\hline
\end{tabular}

* Representative test with poor quality data. 
The measured time histories basically manifest two types of oscillations--low frequency and high frequency. The high-frequency oscillations, as pointed out earlier, are primarily due to instrumentation, and no physical interpretation should be attached to them. The lowfrequency oscillations, however, are real and are partly due to driver response (i.e., sudden acceleration and deceleration of the vehicle due to steering correction to maintain a prescribed circular path) and partly due to surface roughness, inhomogeneity of surface materials, and the fact that the ground is not an ideally flat, level surface. Because of these factors, it is not possible to maintain the vehicle in a. perfect steady-state mode of motion. Therefore, in comparing the field data with model predictions, it is the overall response that must be considered, not the peaks and valleys of the oscillatory records. With this in mind, the comparisons between model predictions and field measurements are very reasonable for the "good tests" (Figures 22-101). For several of these tests, however, the predicted power requirement is lower than the field data (e.g., Figures 30 and 35 ). Examination of the field notes indicated that there was excessive mud buildup inside the tracks of the vehicle for these tests. The mud buildup (which is not simulated by the model) would require additional power to steer the vehicle. The results of test 157 (poor quality data) are presented in Figures 102-106. It is obvious from these figures that except for turning radius the field measurements are of poor quality. Steady-state simulation

14. As pointed out in the previous paragraph, it was not possible to maintain the vehicle in a perfect steady-state mode of motion during the entire test event. However, it is possible to select a small time window for each test where the motion of the vehicle can be reasonably approximated as steady state. The steady-state version of the terrainvehicle interaction model (Appendix A) can then be used to simulate such motions. Such simulations were documented for tests 107-111 (Baladi and Rohani, 1981); for ease of reference, the results are again presented in this report. The time windows and the corresponding test data consisting of the steering ratio $\varepsilon$, vehicle velocity $v$, 
turning radius $R_{o}$, lateral acceleration $v^{2} / R_{o} g$, and total power PT for conducting the steady-state simulations are given in the following tabulation for all of these tests:

\begin{tabular}{|c|c|c|c|c|c|c|c|}
\hline $\begin{array}{l}\text { Test } \\
\text { No. } \\
\end{array}$ & & & $\begin{array}{c}\text { Steering } \\
\text { Ratio } \\
\varepsilon \\
\end{array}$ & $\begin{array}{l}\text { Vehicle } \\
\text { Speed } \\
\text { v, mph } \\
\end{array}$ & $\begin{array}{l}\text { Turning } \\
\text { Radius } \\
\mathrm{R}_{\mathrm{o}} \text {, ft } \\
\end{array}$ & $\begin{array}{c}\text { Lateral } \\
\text { Acceleration } \\
v^{2} / R_{0} g \\
\end{array}$ & $\begin{array}{l}\text { Power } \\
\text { PT, hp }\end{array}$ \\
\hline 107 & 60 & 66 & 1.08 & 24.88 & 155.77 & 0.265 & 210 \\
\hline 108 & 81 & 84 & 1.29 & 14.93 & 63.69 & 0.234 & 199 \\
\hline 109 & $\begin{array}{l}37 \\
68\end{array}$ & $\begin{array}{l}42 \\
71\end{array}$ & $\begin{array}{l}1.12 \\
1.10\end{array}$ & $\begin{array}{l}15.73 \\
18.22\end{array}$ & $\begin{array}{l}103.86 \\
121.26\end{array}$ & $\begin{array}{l}0.16 \\
0.18\end{array}$ & $\begin{array}{l}177 \\
184\end{array}$ \\
\hline 110 & 55 & 59 & 1.26 & 16.08 & 83.40 & 0.21 & 200 \\
\hline 111 & 48 & 51 & 1.57 & 11.79 & 33.74 & 0.275 & 211 \\
\hline
\end{tabular}

Comparisons of the model predictions with experimental data are presented in Figures 107-112 for turning radius versus steering ratio, inner track velocity versus turning radius, outer track velocity versus turning radius, vehicle speed versus turning radius, power requirement versus turning radius, and lateral acceleration versus turning radius, respectively. The model predictions in Figures 108-112 are based on both power cutoff and preliminary stability criteria (see Appendix A). The turning radius-steering ratio relation shown in Figure 107, however, is unique for a given vehicle and soil condition. The power cutoff, as indicated, is controlled by the available power. The preliminary stability criteria are based on (Baladi and Rohani, 1979):

a. Rapid change in the slip velocity of the inner or the outer track.

b. The pivot point falling outside the front edge of the track-ground contact area (i.e., the offset equals $0.5 \mathrm{~L}$ when the center of gravity and center of geometry of the vehicle coincide).

c. Rapid decrease or increase in the turning radius. These stability conditions usually take place at different vehicle velocities. The unstable vehicle velocity is chosen as the minimum 
velocity at which any of these conditions occur. For comparison with the experimental data, the lower vehicle velocity corresponding to either the stability criteria or the power cutoff condition must be selected. As indicated in Figure 110, for the turning radii of $34 \mathrm{ft}$ (test 111) and $64 \mathrm{ft}$ (test 108), stability criteria control the velocity of the vehicle. For the turning radii of $83 \mathrm{ft}$ (test 110), 104 and 121 $\mathrm{ft}$ (test 109), and $156 \mathrm{ft}$ (test 107), the velocity of the vehicle is controlled by the available power. With this in mind, the experimental data in Figures 107-112 compare very favorably with the corresponding model predictions. This fact is particularly true in the case of track velocities and vehicle speed (Figures 108-110). Slight observable differences between the data and model predictions in Figures 107-112 should be expected because of the small deviations in the test conditions from the steady-state mode of motion. 


\section{PART IV: SUMMARY AND RECOMMENDATIONS}

15. In 1978 , the WES developed a mathematical model to predict the steering performance of tracked vehicles. In 1979, field tests of a selected track-laying vehicle were conducted at plowed and unplowed sites on a floodplain north of Redwood, Mississippi, and on a dredge spoil area within the WES reservation at Vicksburg, Mississippi. In this report, the test measurements are compared with calculated results in order to validate the WES terrain-vehicle interaction model for predicting tracked-vehicle performance on soft soils.

16. The floodplain soil at Redwood is a soft, plastic clay (CH); the dredged soil at the WES is a lean, brown clay (CL). Eighty-three direct shear tests were conducted at the test sites. The shear strength of the soils at all three sites was sensitive to the rate of deformation; therefore a soil model with a rate-dependent nonlinear failure envelope was developed. The soil model contains six material constants; values for these constants were defined for each site by fitting the model to the in situ test results. The agreement between the soil model fits and the field data is excellent.

17. A total of 35 circular-turn tests were conducted at the three sites. Due to instrumentation problems, however, only 16 were considered "good tests" for validating the tracked-vehicle model. Theoretical predictions were made for these 16 "good tests," all of which were conducted at the two sites on the floodplain north of Redwood. Theoretical predictions were also made for one test with poor quality data from the dredged soil site. Two types of predictions were made. The first type was made using the transient version of the model; the second was made using the steady-state version of the model.

18. The input driving conditions for the 16 transient predictions consisted of time histories of inner and outer track velocities obtained from actual field measurements. To eliminate artificial highfrequency oscillations and frequent unrealistic crossings of the two track velocity-time histories, the measured track velocities were filtered using Fourier analysis. The model output consisted of time 
histories of vehicle speed, lateral acceleration, power required at the sprockets, and the trajectory of the center of gravity of the vehicle. Comparisons with the corresponding experimental results are very favorable, indicating that the model is capable of predicting the steering performance of tracked vehicles on soft soil.

19. Although the vehicle tests were intended to be constant velocity or steady-state turn tests, the measured track velocities were not constant partly because of (a) driver response (i.e., sudden acceleration or deceleration of the vehicle due to steering correction to maintain a prescribed circular path), and (b) terrain roughness, inhomogeneity of surface materials, plus the fact that the terrain is not an ideally flat, level surface. Therefore, even "steady-state" turn tests should be simulated using the transient version of the model.

20. While it was not possible to maintain the vehicle in a perfect steady-state mode of motion (i.e., constant track velocities) during the entire test event, steady-state conditions did exist for short periods of time during each test. Steady-state calculations were performed for selected time windows in five of the "good tests." The input consisted of a mean radius for each test; the output consisted of a maximum vehicle velocity based on either specified stability criteria or power available at sprockets. These predictions also correlated very favorably with the corresponding test data.

21. To further validate the accuracy and range of application of the model, data are needed from (a) maneuvering tests using a number of tracked vehicles in which the vehicle characteristics (such as weight, track length, and tread) are varied, (b) tests conducted on sloping terrains, and (c) tests conducted on soils other than a soft, plastic clay (e.g., purely cohesionless soils such as dry sand and very hard surfaces such as pavements). 
Baladi, G. Y., and Rohani, B. 1978. "A Mathematical Model of TerrainVehicle Interaction for Predicting the Steering Performance of TrackLaying Vehicles," Proceedings of Sixth International Conference of the International Society for Terrain-Vehicle Systems, Vienna, Austria.

- 1979. "A Terrain-Vehicle Interaction Model for Analysis of Steering Performance of Track-Laying Vehicles," Technical Report GL-79-6, U. S. Army Engineer Waterways Experiment Station, CE, Vicksburg, Miss.

\section{. 1981. "Analysis of Steerability of Tracked Vehicles;}

Theoretical Predictions Versus Field Measurements," Miscellaneous Paper SL-81-3, U. S. Army Engineer Waterways Experiment Station, CE, Vicksburg, Miss.

Bekker, M. B. 1963. The Theory of Land Locomotion, The University of Michigan Press, Ann Arbor, Mich.

Green, C. E. "The Armored Combat Vehicles Technology Program - Results of Soft Soil Tests with the WES M113/2E HOTROD and the General Motors Automotive Test Rig (in preparation)," U. S. Army Engineer Waterways Experiment Station, CE, Vicksburg, Miss.

Hayashi, I. 1975. "Practical Analysis of Tracked Vehicle Steering Depending on Longitudinal Track Slippage," Proceedings, International Society for Terrain-Vehicle Systems Conference, Vol 2, p 493.

Kitano, M., and Jyorzaki, H. 1976. "A Theoretical Analysis of Steerability of Tracked Vehicles," Journal of Terramechanics, International Society for Terrain-Vehicle Systems, Vol 13, No. 4, pp 241-258.

Kitano, M., and Kuma, M. 1977. "An Analysis of Horizontal Plane Motion of Tracked Vehicles," Journal of Terramechanics, International Society for Terrain-Vehicle Systems, Vol 14, No. 4, pp 221-225.

Kondner, R. L. 1963. "Hyperbolic Stress-Strain Response: Cohesive Soils," Journal, Soil Mechahics and Foundation Division, American Society of Civil Engineers, Vol 89, No. SM1, pp 115-143.

Rula, A. A., and Nuttal1, C. J., Jr. 1971. "An Analysis of Ground Mobility Models (ANAMOB)," Technical Report M-71-4, U. S. Army Engineer Waterways Experiment Station, CE, Vicksburg, Miss.

Schreiner, B. G., and Green, C. E. 1980. "Test Areas for HighMobility/Agility (HIMAG) Chassis Test Program," Technical Report GL-807, U. S. Army Engineer Waterways Experiment Station, CE, Vicksburg, Miss. 
Smith, J. L. 1964. "Strength-Moisture-Density Relations of FineGrained Soils in Vehicle Mobility Research," Technical Report 3-639, U. S. Army Engineer Waterways Experiment Station, CE, Vicksburg, Miss.

U. S. Army Engineer Waterways Experiment Station, CE. 1953. "The Unified Soil Classification System," Technical Memorandum No. 3-357, Office of the Chief of Engineers, U. S. Army, Washington, D. C. 
Table 1

Test Site, Soil Type, and

Soil Strength for Each Test Series

\begin{tabular}{|c|c|c|c|c|c|c|c|}
\hline \multirow{2}{*}{$\begin{array}{l}\text { Test } \\
\text { Series } \\
\end{array}$} & \multirow{2}{*}{$\begin{array}{l}\text { Test* } \\
\text { Site }\end{array}$} & \multirow{2}{*}{$\begin{array}{l}\text { Soil } \\
\text { Type }\end{array}$} & \multicolumn{2}{|c|}{$\begin{array}{c}\text { Average } \\
\text { Soil Strength } \\
\text { RCI }\end{array}$} & \multirow{2}{*}{\multicolumn{3}{|c|}{ Date }} \\
\hline & & & $0-6$ in. & 6-12 in. & & & \\
\hline $98-102$ & $7 \mathrm{~A}$ & $\mathrm{CH}$ & 40 & 69 & 1 & June & 1979 \\
\hline $107-111$ & $7 \mathrm{~B}$ & $\mathrm{CH}$ & 24 & 51 & 5 & June & 1979 \\
\hline $117-122$ & $7 \mathrm{~A}$ & $\mathrm{CH}$ & 57 & 93 & 7 & June & 1979 \\
\hline $131-134$ & $7 \mathrm{~A}$ & $\mathrm{CH}$ & 61 & 96 & 8 & June & 1979 \\
\hline $139-144$ & $7 \mathrm{~B}$ & $\mathrm{CH}$ & 54 & 85 & 14 & June & 1979 \\
\hline $157-159$ & 8 & CL & 210 & 198 & 28 & June & 1979 \\
\hline
\end{tabular}

* Site 7A is a plowed ground; site 7B is natural ground; and site 8 is a hydraulic fill.

Table 2

Values of Soil Model Material Constants for

Each Test Series

\begin{tabular}{|c|c|c|c|c|c|c|}
\hline $\begin{array}{c}\text { Test } \\
\text { Series } \\
\end{array}$ & $\begin{array}{c}\text { A } \\
\text { psi }\end{array}$ & $\begin{array}{c}\text { M } \\
\text { psi }\end{array}$ & $\stackrel{N}{1 / p s i}$ & $\begin{array}{r}\mathrm{C}_{\mathrm{d}} \\
\mathrm{psi}\end{array}$ & $\begin{array}{c}\wedge \\
\text { sec/in. }\end{array}$ & $\begin{array}{c}\mathrm{G} \\
\mathrm{psi} / \mathrm{in} .\end{array}$ \\
\hline $98-102$ & 3.5 & 1.94 & 0.3 & 1.25 & 10.0 & 150.0 \\
\hline $107-111$ & 5.0 & 3.75 & 0.22 & 1.10 & 10.0 & 200.0 \\
\hline $117-122$ & 7.8 & 6.36 & 0.1 & 1.75 & 10.0 & 200.0 \\
\hline $131-134$ & 5.4 & 3.65 & 0.23 & 2.14 & 10.0 & 125.0 \\
\hline $139-144$ & 6.5 & 4.94 & 0.09 & 1.75 & 10.0 & 270.0 \\
\hline $157-159$ & 24.0 & 19.94 & 0.02 & 2.64 & 10.0 & 250.0 \\
\hline
\end{tabular}


Table 3

\section{Characteristics of Vehicle Used for Turn Tests}

Weight (W)

Track Length (L)

Track Width (D)

Tread (B)

Height of the center of gravity (H)

Location of the center of gravity measured from the geometrical center of the vehicle $\left(C_{X}\right)$

Distance between two adjacent wheels ( $\ell$ )

Mass moment of inertia $\left(I_{\mathrm{Z}}\right)$

Approach angle $\left(\theta_{\mathrm{a}}\right)$

Departure angle $\left(\theta_{d}\right)$
$=18,0001 \mathrm{~b}_{\mathrm{m}}$

$=105 \mathrm{in}$.

$=15 \mathrm{in}$.

$=90 \mathrm{in}$.

$=35.7 \mathrm{in}$.

$=0 \mathrm{in}$.

$=26.25 \mathrm{in}$.

$=92,0001 b_{f}-i n \cdot-\sec ^{2}$

$=30 \mathrm{deg}$

$=\quad 30 \mathrm{deg}$

Table 4

Rolling Resistance Measured from AC/DC Tests

\section{$\underline{\text { Test Series }}$}

Coefficient of Rolling Resistance

98-102

0.19

$107-111$

0.20

117-122

0.16

$131-134$

0.14

139-144

0.16

157-159

0.125 
Table 5

Numerical Values of the Coefficients $A_{i}$ and $B_{i}$

(Equation 1) for Tests 99 and 100

\begin{tabular}{|c|c|c|c|c|c|c|c|c|}
\hline \multirow{3}{*}{$\begin{array}{l}\mathrm{I} \\
\mathrm{N} \\
\mathrm{D} \\
\mathrm{E} \\
\mathrm{X} \\
\mathrm{i}\end{array}$} & \multicolumn{4}{|c|}{ TEST NUMBER 99} & \multicolumn{4}{|c|}{ TEST NUMBER 100} \\
\hline & \multicolumn{2}{|c|}{ OUTER TRACK } & \multicolumn{2}{|c|}{ INNER TRACK } & \multicolumn{2}{|c|}{ OUTER TRACK } & \multicolumn{2}{|c|}{ INNER TRACK } \\
\hline & $A_{i}$ & $\mathrm{~B}_{i}$ & $\mathrm{~A}_{i}$ & $\mathrm{~B}_{i}$ & $\mathrm{~A}_{i}$ & $B_{i}$ & $\mathrm{~A}_{i}$ & $\mathrm{~B}_{i}$ \\
\hline 1 & 13.578 & 0 & 9.2699 & 0 & 10.543 & 0 & 5.652 & 0 \\
\hline 2 & 69.935 & -55.671 & 46.269 & -42.318 & 59.974 & -37.856 & 35.596 & -25.739 \\
\hline 3 & 18.701 & 2.694 & 14.431 & 1.706 & 6.788 & -15.614 & 4.092 & -8.648 \\
\hline 4 & 4.137 & -4.374 & 2.738 & -3.520 & 5.167 & -6.928 & 3.339 & -3.914 \\
\hline 5 & 6.782 & -1.595 & 5.189 & -1.095 & 1.060 & -6.089 & 0.605 & -3.669 \\
\hline 6 & 2.969 & 0.088 & 1.300 & -0.344 & 2.457 & -2.182 & 1.378 & -1.522 \\
\hline 7 & 1.432 & -2.664 & 1.120 & -1.196 & -0.073 & -3.239 & -0.097 & -1.725 \\
\hline 8 & 1.704 & -0.314 & 1.347 & -0.283 & 0.270 & -1.512 & 0.583 & -1.013 \\
\hline 9 & 0.807 & 0.426 & 0.626 & 0.330 & -0.847 & -0.421 & -0.470 & -0.492 \\
\hline 10 & 0.735 & -0.497 & 0.416 & -0.420 & 0.151 & -1.037 & -0.115 & -0.783 \\
\hline 11 & 0.980 & -0.070 & 0.823 & -0.093 & -0.544 & -0.120 & -0.151 & -0.014 \\
\hline 12 & 0.889 & -0.014 & 0.491 & -0.229 & -0.330 & -0.354 & -0.191 & -0.427 \\
\hline 13 & 0.675 & -0.798 & 0.131 & -0.043 & -0.468 & 0.010 & -0.034 & -0.189 \\
\hline 14 & 0.251 & -0.070 & 0.649 & 0.242 & -0.232 & -0.142 & -0.193 & -0.148 \\
\hline 15 & 0.377 & -0.155 & 0.492 & -0.043 & -0.246 & 0.018 & -0.418 & -0.045 \\
\hline 16 & 0.274 & -0.181 & 0.284 & -0.195 & -0.119 & 0.073 & 0.007 & 0.181 \\
\hline 17 & 0.408 & 0.067 & 0.378 & -0.077 & -0.106 & 0.144 & -0.065 & -0.010 \\
\hline 18 & 0.245 & -0.050 & 0.231 & -0.017 & 0.078 & 0.103 & 0.089 & -0.145 \\
\hline 19 & 0.563 & 0.050 & 0.372 . & -0.397 & -0.060 & -0.080 & -0.163 & 0.103 \\
\hline 20 & 0.407 & -0.311 & 0.013 & -0.047 & 0.154 & -0.077 & 0.084 & -0.023 \\
\hline 21 & 0.248 & -0.004 & 0.149 & -0.071 & 0.081 & -0.123 & -0.091 & -0.032 \\
\hline
\end{tabular}


Table 6

Numerical Values of the Coefficients $A_{i}$ and $B_{i}$

(Equation 1) for Tests 101 and 102

\begin{tabular}{|c|c|c|c|c|c|c|c|c|}
\hline \multirow{3}{*}{$\begin{array}{l}\mathrm{I} \\
\mathrm{N} \\
\mathrm{D} \\
\mathrm{E} \\
\mathrm{X} \\
\mathrm{i}\end{array}$} & \multicolumn{4}{|c|}{ TEST NUMBER 101} & \multicolumn{4}{|c|}{ TEST NUMBER 102} \\
\hline & \multicolumn{2}{|c|}{ OUTER TRACK } & \multicolumn{2}{|c|}{ INNER TRACK } & \multicolumn{2}{|c|}{ OUTER TRACK } & \multicolumn{2}{|c|}{ INNER TRACK } \\
\hline & $A_{i}$ & $\mathrm{~B}_{\mathrm{i}}$ & $\mathrm{A}_{\mathrm{i}}$ & $\mathrm{B}_{\mathrm{i}}$ & $\mathrm{A}_{\mathrm{i}}$ & $\mathrm{B}_{i}$ & $\mathrm{~A}_{\mathbf{i}}$ & $\mathrm{B}_{i}$ \\
\hline 1 & 13.619 & 0 & 10.038 & 0 & 13.718 & 0 & 11.961 & 0 \\
\hline 2 & 61.520 & $-62 \cdot 582$ & 50.982 & -51.107 & 51.396 & $-84 \cdot 309$ & 48.839 & -76.819 \\
\hline 3 & 17.260 & 4.926 & 15.341 & 4.033 & 41.238 & -7.026 & 38.371 & -6.153 \\
\hline 4 & 7.404 & -6.457 & 6.167 & -5.522 & -0.347 & -3.247 & 0.497 & -3.042 \\
\hline 5 & 5.600 & -6.986 & 4.318 & -5.302 & 3.595 & 0.988 & 2.938 & 0.989 \\
\hline 6 & 1.030 & -0.650 & 1.255 & -0.270 & 4.954 & -3.098 & 4.736 & -2.617 \\
\hline 7 & 1.387 & -2.295 & 0.994 & -1.951 & 4.447 & -0.446 & 4.196 & -0.230 \\
\hline 8 & 1.717 & 0.200 & 1.478 & 0.160 & -0.402 & -1.386 & -0.328 & -1.412 \\
\hline 9 & 1.354 & 0.086 & 1.201 & -0.040 & 1.703 & -0.257 & 1.571 & -0.097 \\
\hline 10 & 1.516 & -1.322 & 0.920 & -1.055 & 2.107 & -0.573 & 1.936 & -0.479 \\
\hline 11 & 0.853 & -1.094 & 0.832 & -0.685 & 0.784 & -0.365 & 0.693 & -0.297 \\
\hline 12 & 0.655 & -0.267 & 0.385 & -0.237 & 0.712 & -0.410 & 0.590 & -0.172 \\
\hline 13 & 0.324 & -0.339 & 0.329 & -0.164 & 0.690 & -0.561 & 0.571 & -0.490 \\
\hline 14 & 0.579 & -0.043 & 0.567 & -0.066 & 0.859 & 0.028 & 0.852 & 0.002 \\
\hline 15 & 0.702 & -0.255 & 0.361 & -0.413 & 0.701 & -0.169 & 0.569 & -0.141 \\
\hline 16 & 0.220 & -0.509 & 0.063 & -0.282 & 0.498 & -0.254 & 0.431 & -0.228 \\
\hline 17 & 0.151 & -0.405 & 0.115 & -0.385 & 0.379 & -0.326 & 0.368 & -0.340 \\
\hline 18 & 0.115 & -0.179 & -0.005 & 0.069 & 0.386 & -0.393 & 0.425 & -0.399 \\
\hline 19 & 0.074 & 0.045 & 0.174 & 0.192 & 0.486 & -0.130 & 0.375 & -0.053 \\
\hline 20 & 0.063 & -0.080 & 0.347 & -0.020 & 0.276 & -0.083 & 0.208 & -0.149 \\
\hline 21 & 0.207 & 0.008 & 0.222 & -0.130 & 0.116 & -0.264 & 0.162 & -0.160 \\
\hline
\end{tabular}


Table 7

Numerical Values of the Coefficients $A_{i}$ and $B_{i}$ (Equation 1) for Tests 107 and 108

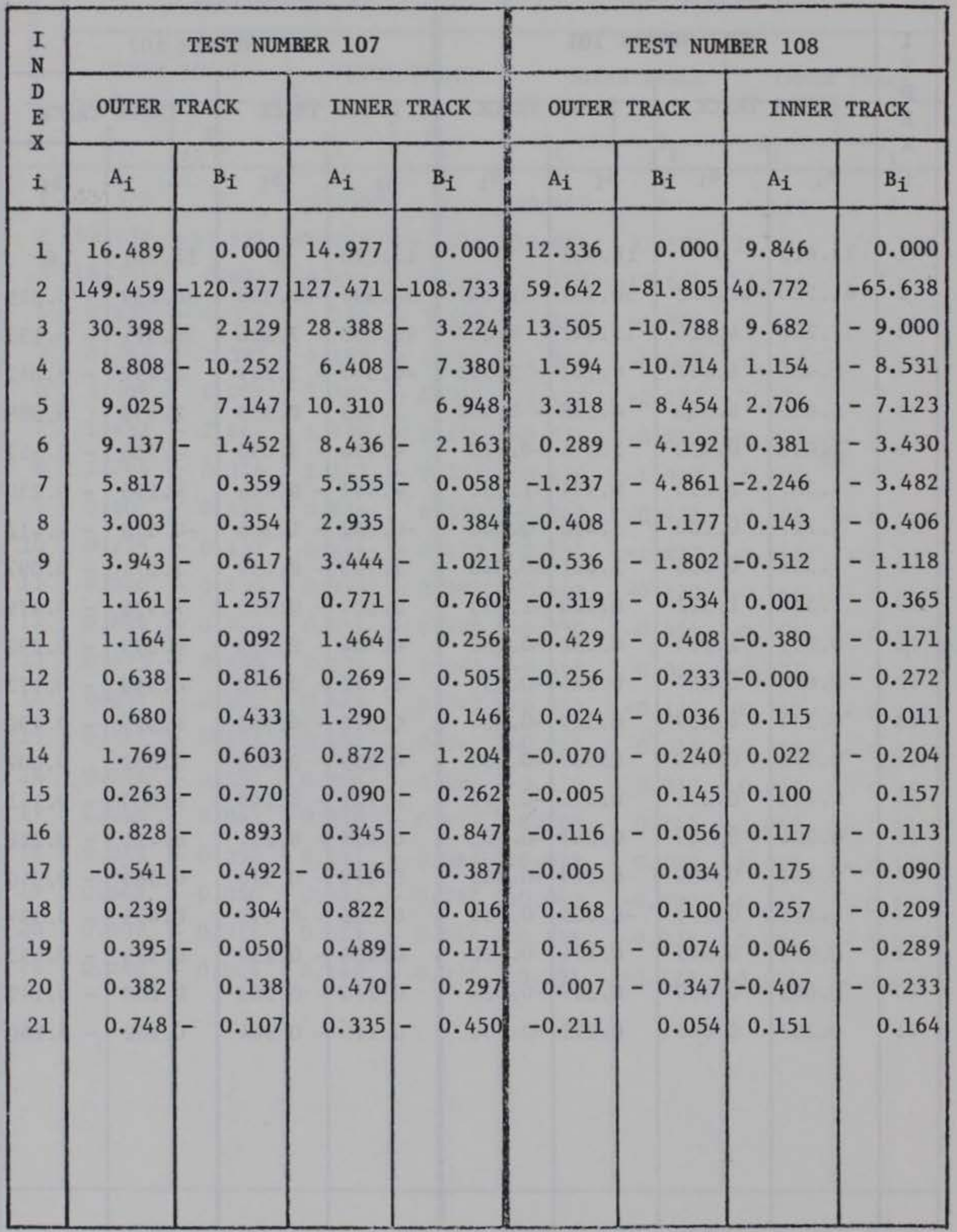


Table 8

Numerical Values of the Coefficients $A_{i}$ and $B_{i}$

(Equation 1) for Tests 109 and 110

\begin{tabular}{|c|c|c|c|c|c|c|c|c|}
\hline I & \multicolumn{4}{|c|}{ TEST NUMBER 109} & \multicolumn{4}{|c|}{ TEST NUMBER 110} \\
\hline E & OUTER & TRACK & INNER & TRACK & OUTER & TRACK & INNER & TRACK \\
\hline i. & $A_{i}$ & $\mathrm{~B}_{\mathrm{i}}$ & $A_{i}$ & $\mathrm{~B}_{\mathrm{i}}$ & $A_{i}$ & $\mathrm{~B}_{\mathbf{i}}$ & $\mathrm{A}_{i}$ & $\mathrm{~B}_{\mathrm{i}}$ \\
\hline 1 & 14.031 & 0 & 12.784 & 0 & 14.797 & 0 & 12.690 & 0 \\
\hline 2 & 52.868 & -70.877 & 47.416 & -62.742 & 56.488 & -56.478 & 43.883 & -45.917 \\
\hline 3 & 20.035 & -12.873 & 17.624 & -12.932 & 21.489 & -4.846 & 18.416 & -5.903 \\
\hline 4 & 7.470 & -3.498 & 7.394 & -3.761 & 6.959 & -2.340 & 5.494 & -3.293 \\
\hline 5 & 3.473 & -6.441 & 2.413 & -5.862 & 4.732 & -1.224 & 4.645 & -1.795 \\
\hline 6 & 1.486 & -3.986 & 1.340 & -3.896 & 4.012 & -3.666 & 1.470 & -3.381 \\
\hline 7 & 0.388 & -2.978 & 0.254 & -2.572 & 0.734 & -1.285 & 0.864 & -0.676 \\
\hline 8 & 0.277 & -2.222 & 0.061 & -1.891 & 1.061 & -1.524 & 0.710 & -1.479 \\
\hline 9 & -0.210 & -1.441 & -0.114 & -1.113 & 1.140 & -1.151 & 0.464 & -1.028 \\
\hline 10 & -0.352 & -1.389 & -0.617 & -1.135 & -0.237 & -1.469 & -0.226 & -0.775 \\
\hline 11 & -0.263 & -1.255 & -0.476 & -1.186 & 0.296 & 0.046 & 0.561 & -0.000 \\
\hline 12 & -0.411 & -0.543 & -0.492 & -0.451 & 0.493 & -0.345 & 0.409 & -0.458 \\
\hline 13 & -0.197 & -0.298 & -0.034 & -0.378 & 0.358 & -0.200 & 0.244 & -0.297 \\
\hline 14 & -0.333 & -0.603 & -0.326 & -0.448 & 0.017 & -0.314 & 0.083 & -0.113 \\
\hline 15 & 0.026 & -0.425 & -0.139 & -0.436 & 0.305 & -0.069 & 0.305 & -0.281 \\
\hline 16 & -0.391 & -0.496 & -0.373 & -0.182 & 0.243 & -0.233 & 0.073 & -0.341 \\
\hline 17 & -0.050 & -0.048 & -0.028 & -0.272 & 0.153 & -0.264 & 0.062 & -0.235 \\
\hline 18 & -0.236 & -0.118 & -0.089 & -0.079 & 0.185 & -0.300 & 0.079 & -0.216 \\
\hline 19 & 0.121 & -0.234 & -0.135 & -0.278 & 0.141 & 0.017 & 0.154 & -0.144 \\
\hline 20 & -0.152 & -0.285 & -0.099 & -0.027 & 0.233 & -0.397 & -0.090 & -0.258 \\
\hline 21 & -0.076 & -0.092 & -0.003 & -0.069 & 0.006 & -0.227 & -0.012 & -0.087 \\
\hline
\end{tabular}


Table 9

Numerical Values of the Coefficients $A_{i}$ and $B_{i}$ (Equation 1) for Tests 111 and 117

\begin{tabular}{|c|c|c|c|c|c|c|c|c|}
\hline \multirow{3}{*}{$\begin{array}{l}\mathrm{I} \\
\mathrm{N} \\
\mathrm{D} \\
\mathrm{E} \\
\mathrm{X} \\
\mathrm{i}\end{array}$} & \multicolumn{4}{|c|}{ TEST NUMBER 111} & \multicolumn{4}{|c|}{ TEST NUMBER 117} \\
\hline & \multicolumn{2}{|c|}{ OUTER TRACK } & \multicolumn{2}{|c|}{ INNER TRACK } & \multicolumn{2}{|c|}{ OUTER TRACK } & \multicolumn{2}{|c|}{ INNER TRACK } \\
\hline & $A_{i}$ & $\mathrm{~B}_{\mathrm{i}}$ & $A_{i}$ & $B_{i}$ & $A_{i}$ & $\mathrm{~B}_{\mathrm{i}}$ & $A_{i}$ & $B_{i}$ \\
\hline 1 & 12.484 & 0 & 8.201 & 0 & 18.062 & 0 & 16.643 & 0 \\
\hline 2 & 27.350 & -33.928 & 13.408 & -21.948 & 124.848 & -134.382 & 111.637 & -119.956 \\
\hline 3 & 8.983 & -3.087 & 5.617 & -2.509 & 38.878 & 2.946 & 35.272 & 2.856 \\
\hline 4 & 2.492 & -3.579 & 1.134 & -2.442 & 4.516 & 1.541 & 5.043 & 1.924 \\
\hline 5 & 2.235 & -3.012 & 1.840 & -2.127 & 1.554 & -4.525 & 2.061 & -2.873 \\
\hline 6 & 1.641 & -1.656 & 1.420 & -0.836 & 11.284 & 10.782 & 10.923 & 8.031 \\
\hline 7 & 0.835 & -1.092 & 0.316 & -1.023 & 3.952 & -5.554 & 2.834 & -4.562 \\
\hline 8 & 0.727 & -0.535 & 0.604 & -0.374 & 4.976 & -1.941 & 4.349 & -1.730 \\
\hline 9 & 0.365 & -0.568 & 0.319 & -0.277 & 1.823 & 0.120 & 1.789 & 0.213 \\
\hline 10 & 0.231 & -0.619 & 0.093 & -0.341 & 0.452 & -0.366 & 0.608 & 0.122 \\
\hline 11 & 0.306 & -0.321 & 0.134 & -0.010 & 3.198 & 0.596 & 3.228 & -0.225 \\
\hline 12 & 0.120 & 0.110 & a. 315 & 0.063 & 1.509 & -1.189 & 1.027 & -0.971 \\
\hline 13 & 0.577 & -0.147 & 0.223 & -0.220 & 1. 353 & -0.482 & 1.170 & -0.464 \\
\hline 14 & 0.386 & 0.001 & 0.266 & -0.115 & 0.888 & -0.279 & 0.874 & -0.187 \\
\hline 15 & 0.250 & -0.118 & 0.074 & -0.165 & 0.882 & -0.340 & 0.872 & -0.140 \\
\hline 16 & 0.323 & -0.022 & 0.184 & -0.149 & 0.847 & -0.157 & 0.970 & -0.443 \\
\hline 17 & 0.443 & -0.173 & 0.155 & -0.261 & 0.863 & -0.404 & 0.526 & -0.321 \\
\hline 18 & 0.032 & -0.364 & -0.098 & -0.068 & 0.549 & -0.340 & 0.421 & -0.306 \\
\hline 19 & 0.070 & -0.037 & 0.042 & -0.055 & 0.369 & -0.238 & 0.323 & -0.173 \\
\hline 20 & 0.033 & -0.134 & 0.026 & -0.108 & 0.412 & -0.083 & 0.377 & -0.046 \\
\hline 21 & 0.013 & -0.104 & 0.007 & -0.004 & 0.345 & -0.098 & 0.488 & -0.221 \\
\hline
\end{tabular}


Table 10

Numerical Values of the Coefficients $A_{i}$ and $B_{i}$

(Equation 1) for Tests 118 and 133)

\begin{tabular}{|c|c|c|c|c|c|c|c|c|}
\hline \multirow{3}{*}{$\begin{array}{l}\mathrm{I} \\
\mathrm{N} \\
\mathrm{D} \\
\mathrm{E} \\
\mathrm{X} \\
\mathrm{i}\end{array}$} & \multicolumn{4}{|c|}{ TEST NUMBER 118} & \multicolumn{4}{|c|}{ TEST NUMBER 133} \\
\hline & \multicolumn{2}{|c|}{ OUTER TRACK } & \multicolumn{2}{|c|}{ INNER TRACK } & \multicolumn{2}{|c|}{ OUTER TRACK } & \multicolumn{2}{|c|}{ INNER TRACK } \\
\hline & $A_{i}$ & $\mathrm{~B}_{i}$ & $A_{i}$ & $\mathrm{~B}_{i}$ & $A_{i}$ & $\mathrm{~B}_{i}$ & $A_{i}$ & $\mathrm{~B}_{i}$ \\
\hline 1 & 12.758 & 0 & 11.401 & 0 & 14.056 & 0 & 12.939 & 0 \\
\hline 2 & 130.463 & $-64 \cdot 318$ & 113.926 & -56.462 & 49.841 & -93.290 & 38.392 & -76.958 \\
\hline 3 & 29.840 & -5.802 & 27.198 & -5.776 & 9.315 & -15.959 & 7.855 & -13.042 \\
\hline 4 & 12.431 & -9.290 & 10.797 & -7.559 & -3.691 & -9.206 & -2.026 & -7.312 \\
\hline 5 & 6.003 & 3.358 & 6.352 & 2.520 & 0.018 & -4.550 & -0.068 & -4.678 \\
\hline 6 & 6.270 & -1.439 & 5.147 & -1.469 & -2.633 & -2.469 & -2.398 & -1.895 \\
\hline 7 & 2.148 & -0.672 & 2.166 & -0.376 & -1.196 & -1.079 & -0.983 & -0.788 \\
\hline 8 & 1.651 & -2.044 & 1.173 & -1.715 & -0.901 & -0.070 & -0.550 & -0.050 \\
\hline 9 & -0.363 & -0.838 & 0.102 & -0.634 & 0.011 & -0.033 & -0.060 & -0.277 \\
\hline 10 & 1.951 & -0.329 & 1.698 & -0.669 & 0.254 & -0.529 & -0.033 & -0.427 \\
\hline 11 & 0.804 & -1.087 & 0.653 & -0.903 & 0.096 & -0.340 & 0.073 & -0.277 \\
\hline 12 & 0.819 & -0.768 & 0.670 & -0.779 & -0.021 & -0.486 & 0.001 & -0.411 \\
\hline 13 & 0.285 & -0.543 & 0.297 & -0.365 & 0.243 & -0.177 & 0.164 & -0.467 \\
\hline 14 & 0.248 & -0.411 & 0.295 & -0.448 & -0.092 & -0.574 & -0.280 & -0.291 \\
\hline 15 & 0.254 & -0.447 & 0.114 & -0.385 & -0.122 & -0.179 & 0.018 & -0.082 \\
\hline 16 & -0.016 & -0.273 & 0.000 & -0.290 & -0.151 & -0.155 & -0.019 & -0.082 \\
\hline 17 & 0.081 & -0.374 & 0.082 & -0.285 & 0.093 & -0.063 & 0.027 & -0.249 \\
\hline 18 & 3.104 & -0.231 & 0.105 & -0.261 & -0.067 & -0.251 & -0.069 & -0.008 \\
\hline 19 & 0.013 & -0.227 & -0.013 & -0.178 & 0.005 & -0.101 & 0.132 & -0.093 \\
\hline 20 & 0.107 & -0.140 & 0.020 & -0.177 & 0.012 & -0.169 & 4.604 & -0.147 \\
\hline 21 & 0.010 & -0.118 & 0.029 & -0.090 & -0.012 & -0.177 & -0.014 & -0.164 \\
\hline
\end{tabular}


Table 11

Numerical Values of the Coefficients $A_{i}$ and $B_{i}$

(Equation 1) for Tests 134 and 139

\begin{tabular}{|c|c|c|c|c|c|c|c|c|}
\hline I & \multicolumn{4}{|c|}{ TEST NUMBER 134} & \multicolumn{4}{|c|}{ TEST NUMBER 139} \\
\hline $\mathrm{E}$ & \multicolumn{2}{|c|}{ OUTER TRACK } & \multicolumn{2}{|c|}{ INNER TRACK } & \multicolumn{2}{|c|}{ OUTER TRACK } & \multicolumn{2}{|c|}{ INNER TRACK } \\
\hline$i$ & $\mathrm{~A}_{i}$ & $\mathrm{~B}_{i}$ & $\mathrm{~A}_{i}$ & $\mathrm{~B}_{i}$ & $A_{i}$ & $\mathrm{~B}_{i}$ & $A_{i}$ & $\mathrm{~B}_{i}$ \\
\hline 1 & 11.273 & 0 & 8.009 & 0 & 21.834 & 0 & 20.196 & 0 \\
\hline 2 & 19.954 & -28.321 & 9.413 & -17.684 & 122.426 & -95.596 & 111.791 & 89.443 \\
\hline 3 & 8.482 & -2.735 & 4.809 & -2.295 & 28.839 & -2.653 & 26.801 & -2.998 \\
\hline 4 & 2.604 & -2.526 & 1.361 & -1.539 & 13.627 & -8.559 & 12.271 & -7.919 \\
\hline 5 & 2.435 & -1.710 & 1.246 & -0.831 & 4.825 & -4.434 & 4.396 & -3.921 \\
\hline 6 & 1.311 & -0.589 & 1.097 & -0.203 & 3.692 & -1.132 & 3.395 & -1.094 \\
\hline 7 & 1.060 & -0.709 & 0.677 & -0.494 & 2.761 & -2.631 & 2.484 & -2.326 \\
\hline 8 & 0.854 & -0.600 & 0.552 & -0.247 & 1.110 & -1.663 & 1.067 & -1.418 \\
\hline 9 & 0.700 & -0.341 & 0.410 & -0.166 & 1.111 & -0.398 & 1.138 & -0.391 \\
\hline 10 & 0.284 & -0.379 & 0.371 & -0.163 & 1.470 & -0.906 & 1.344 & -0.904 \\
\hline 11 & 0.280 & -0.166 & 0.163 & -0.220 & 0.676 & -0.773 & 0.659 & -0.816 \\
\hline 12 & 0.167 & -0.281 & 0.068 & -0.129 & 0.795 & -0.591 & 0.687 & -0.647 \\
\hline 13 & 0.267 & -0.114 & 0.175 & -0.209 & 0.411 & -0.422 & 0.403 & -0.347 \\
\hline 14 & 0.066 & -0.221 & 0.001 & 0.025 & 0.393 & -0.286 & 0.414 & -0.345 \\
\hline 15 & 0.050 & -0.041 & 0.169 & -0.014 & 0.411 & -0.264 & 0.347 & -0.264 \\
\hline 16 & 0.157 & -0.134 & 0.006 & -0.038 & 0.471 & -0.380 & 0.327 & -0.409 \\
\hline 17 & 0.084 & -0.100 & 0.081 & -0.042 & 0.176 & -0.326 & 0.099 & -0.194 \\
\hline 18 & 0.009 & -0.156 & 0.086 & 0.028 & 0.241 & -0.224 & 0.171 & -0.149 \\
\hline 19 & 0.053 & 0.002 & 0.054 & -0.107 & 0.259 & -0.274 & 0.211 & -0.236 \\
\hline 20 & 0.092 & -0.123 & 0.008 & 0.003 & 0.234 & -0.226 & 0.153 & -0.215 \\
\hline 21 & 0.004 & -0.092 & 0.024 & -0.025 & 0.091 & -0.228 & 0.080 & -0.114 \\
\hline
\end{tabular}


Table 12

Numerical Values of the Coefficients $A_{i}$ and $B_{i}$

(Equation 1) for Tests 140 and 141

\begin{tabular}{|c|c|c|c|c|c|c|c|c|}
\hline \multirow{3}{*}{$\begin{array}{l}\mathrm{I} \\
\mathrm{N} \\
\mathrm{D} \\
\mathrm{E} \\
\mathrm{X} \\
\mathrm{i}\end{array}$} & \multicolumn{4}{|c|}{ TEST NUMBER 140} & \multicolumn{4}{|c|}{ TEST NUMBER 141} \\
\hline & \multicolumn{2}{|c|}{ OUTER TRACK } & \multicolumn{2}{|c|}{ INNER TRACK } & \multicolumn{2}{|c|}{ OUTER TRACK } & \multicolumn{2}{|c|}{ INNER TRACK } \\
\hline & $\mathrm{A}_{i}$ & $\mathrm{~B}_{i}$ & $\mathrm{~A}_{i}$ & $\mathrm{~B}_{i}$ & $A_{i}$ & $\mathrm{~B}_{i}$ & $\mathrm{~A}_{i}$ & $\mathrm{~B}_{i}$ \\
\hline 1 & 19.704 & 0 & 17.737 & 0 & 13.798 & 0 & 11.291 & 0 \\
\hline 2 & 74.107 & -54.121 & 64.482 & -48.583 & 48.173 & -90.447 & 36.294 & -73.407 \\
\hline 3 & 17.046 & -0.236 & 15.305 & -0.309 & 9.322 & -12.514 & 7.749 & -10.096 \\
\hline 4 & 7.022 & -3.780 & 6.295 & -3.159 & -4.608 & -8.309 & -3.391 & -6.502 \\
\hline 5 & 4.384 & -2.138 & 4.000 & -1.987 & -4.030 & -4.228 & -3.187 & -3.468 \\
\hline 6 & 1.940 & -1.793 & 1.670 & -1.649 & -2.995 & -3.521 & -2.532 & -3.063 \\
\hline 7 & 2.320 & -1.831 & 1.893 & -1.758 & -1.108 & -1.814 & -0.990 & -1.399 \\
\hline 8 & 0.626 & -1.510 & 0.439 & -1.028 & 0.117 & 0.030 & 0.174 & -0.078 \\
\hline 9 & 0.880 & -0.209 & 1.073 & -0.230 & 0.135 & 0.417 & 0.288 & 0.186 \\
\hline 10 & 0.866 & -0.739 & 0.701 & -0.780 & 0.231 & 0.272 & 0.196 & 0.053 \\
\hline 11 & 0.480 & -0.637 & 0.349 & -0.584 & 0.395 & -0.323 & 0.106 & -0.388 \\
\hline 12 & 0.409 & -0.527 & 0.298 & -0.528 & -0.170 & -0.707 & -0.339 & -0.307 \\
\hline 13 & 0.352 & -0.325 & 0.326 & -0.354 & 0.038 & -0.252 & 0.035 & -0.246 \\
\hline 14 & 0.328 & -0.415 & 0.272 & -0.379 & 0.039 & -0.397 & 0.008 & -0.340 \\
\hline 15 & 0.220 & -0.188 & 0.257 & -0.237 & 0.048 & -0.169 & 0.048 & -0.198 \\
\hline 16 & 0.287 & -0.269 & 0.139 & -0.326 & -0.020 & -0.115 & 0.024 & 0.022 \\
\hline 17 & 0.141 & -0.246 & 0.051 & -0.166 & -0.089 & -0.190 & -0.058 & -0.086 \\
\hline 18 & 0.099 & -0.153 & 0.123 & -0.142 & -0.004 & 0.049 & 0.114 & -0.027 \\
\hline 19 & 0.042 & -0.169 & 0.038 & -0.103 & 0.102 & -0.129 & 0.036 & -0.217 \\
\hline 20 & 0.102 & -0.081 & 0.113 & -0.080 & 0.028 & -0.089 & 0.012 & -0.103 \\
\hline 21 & 0.099 & -0.116 & 0.037 & -0.126 & 0.007 & -0.030 & 0.065 & -0.094 \\
\hline
\end{tabular}


Table 13

Numerical Values of the Coefficients

$\mathrm{A}_{i}$ and $\mathrm{B}_{i}$ (Equation 1) for Test 157

\begin{tabular}{|c|c|c|c|c|}
\hline $\begin{array}{l}\mathrm{I} \\
\mathrm{N}\end{array}$ & \multicolumn{4}{|c|}{ TEST NUMBER 157} \\
\hline E & \multicolumn{2}{|c|}{ OUTER TRACK } & \multicolumn{2}{|c|}{ INNER TRACK } \\
\hline$i$ & $A_{i}$ & $\mathrm{~B}_{i}$ & $A_{i}$ & $\mathrm{~B}_{i}$ \\
\hline 1 & 8.932 & 0 & 13.486 & 0 \\
\hline 2 & 26.942 & -29.636 & 52.027 & -50.415 \\
\hline 3 & 10.103 & -2.030 & 15.711 & -3.422 \\
\hline 4 & 2.776 & -1.681 & 3.711 & -3.482 \\
\hline 5 & 1.926 & -2.079 & 2.876 & -3.277 \\
\hline 6 & 1.078 & -0.708 & 1.322 & -1.058 \\
\hline 7 & 0.539 & -1.060 & 0.625 & -1.443 \\
\hline 8 & 0.443 & -0.887 & 0.721 & -1.039 \\
\hline 9 & 0.044 & -0.874 & 0.269 & -0.964 \\
\hline 10 & -0.150 & -0.107 & 0.057 & -0.376 \\
\hline 11 & 0.022 & -0.117 & 0.052 & -0.383 \\
\hline 12 & 0.032 & -0.082 & 0.034 & -0.205 \\
\hline 13 & 0.014 & -0.059 & 0.010 & -0.221 \\
\hline 14 & 0.156 & -0.179 & -0.032 & -0.095 \\
\hline 15 & 0.073 & -0.210 & 0.109 & -0.095 \\
\hline 16 & -0.052 & -0.013 & 0.134 & -0.041 \\
\hline 17 & 0.040 & -0.059 & 0.071 & -0.071 \\
\hline 18 & 0.011 & 0.024 & 0.089 & -0.123 \\
\hline 19 & 0.085 & -0.092 & 0.047 & -0.083 \\
\hline 20 & 0.080. & 0.055 & 0.035 & -0.166 \\
\hline 21 & 0.224 & -0.035 & -0.004 & -0.075 \\
\hline
\end{tabular}




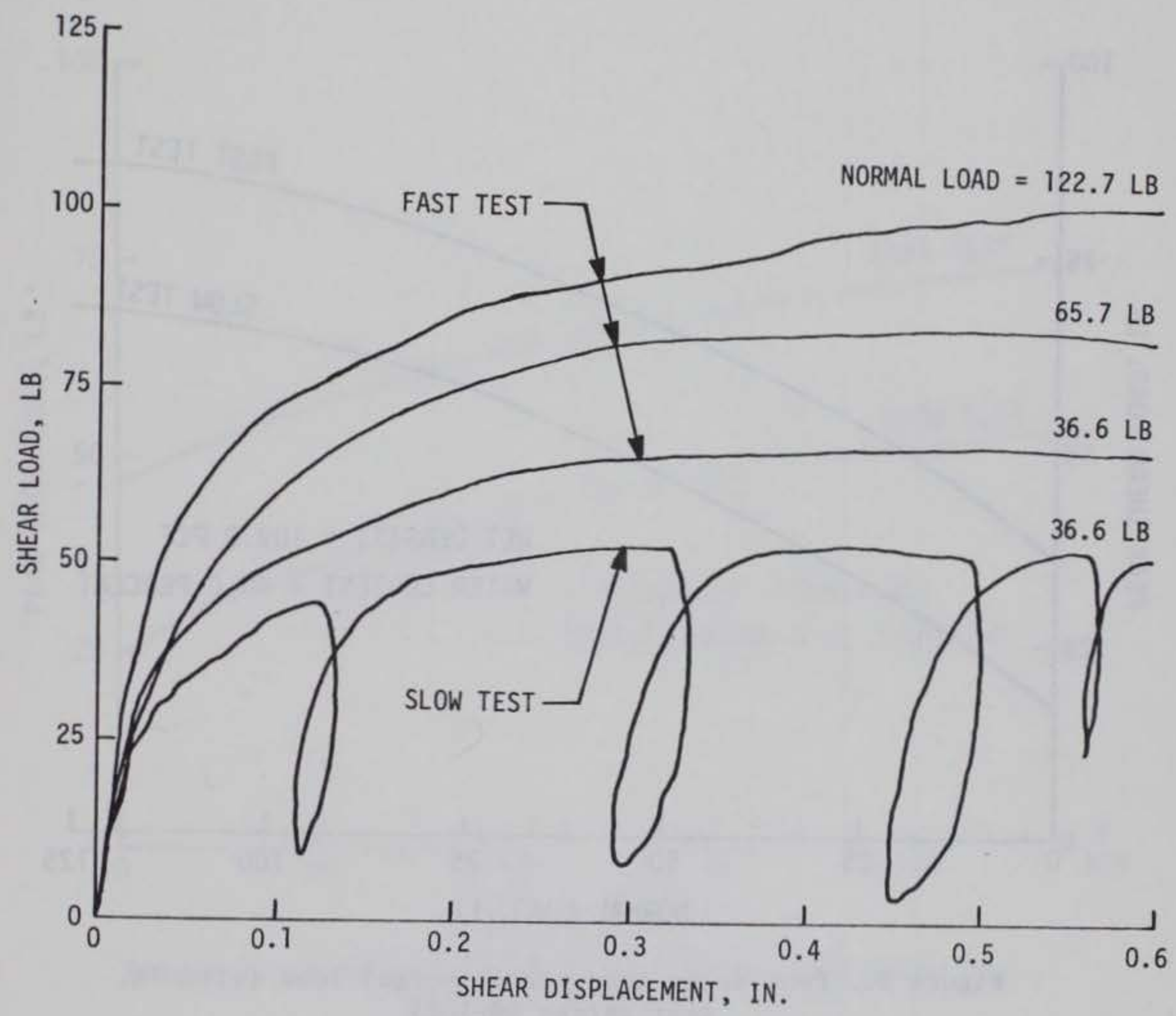

Figure 1. Typical load deformation curves obtained from the direct shear device (site 7A) 


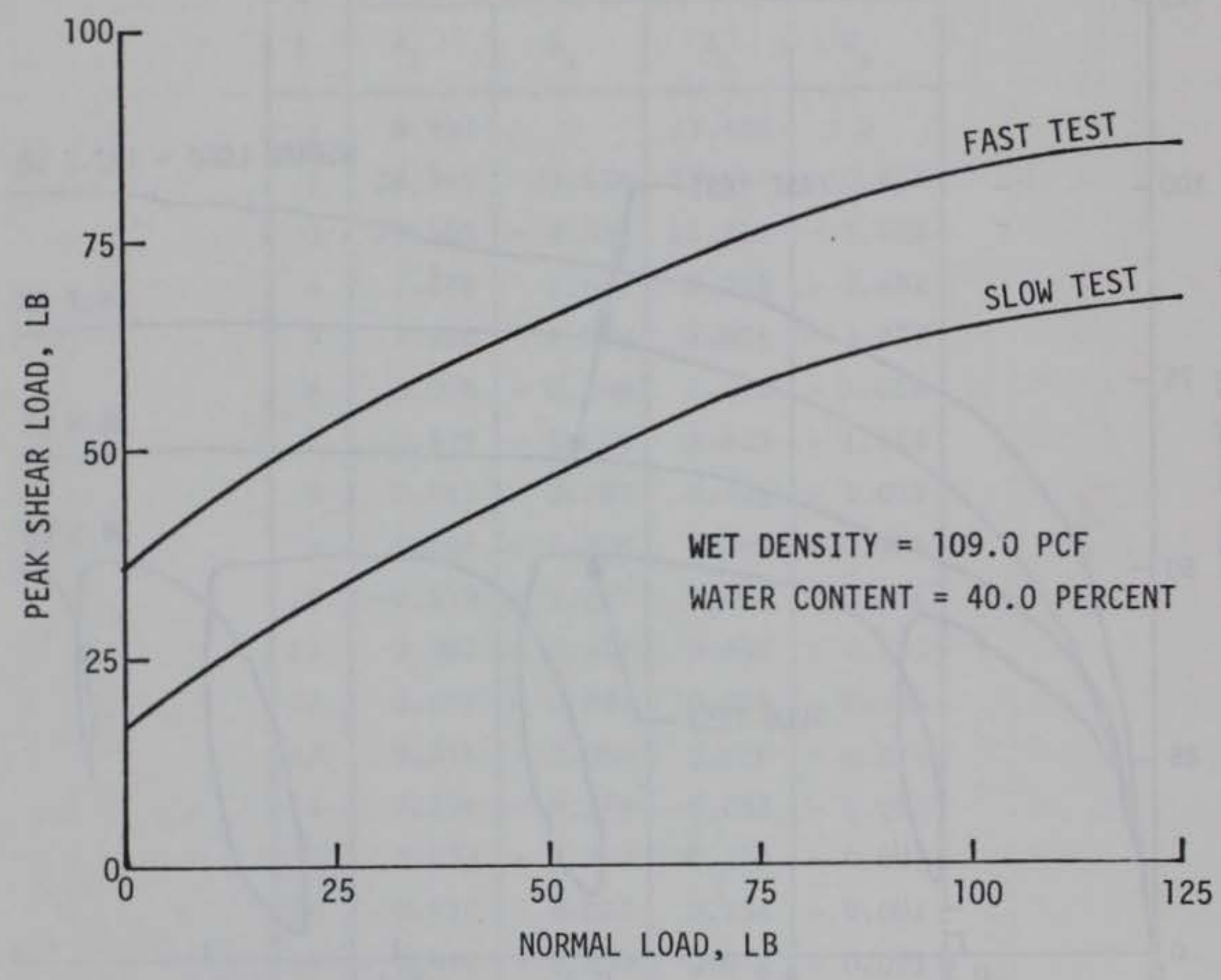

Figure 2. Peak shear load versus normal load (site 7A, test series 98-102) 


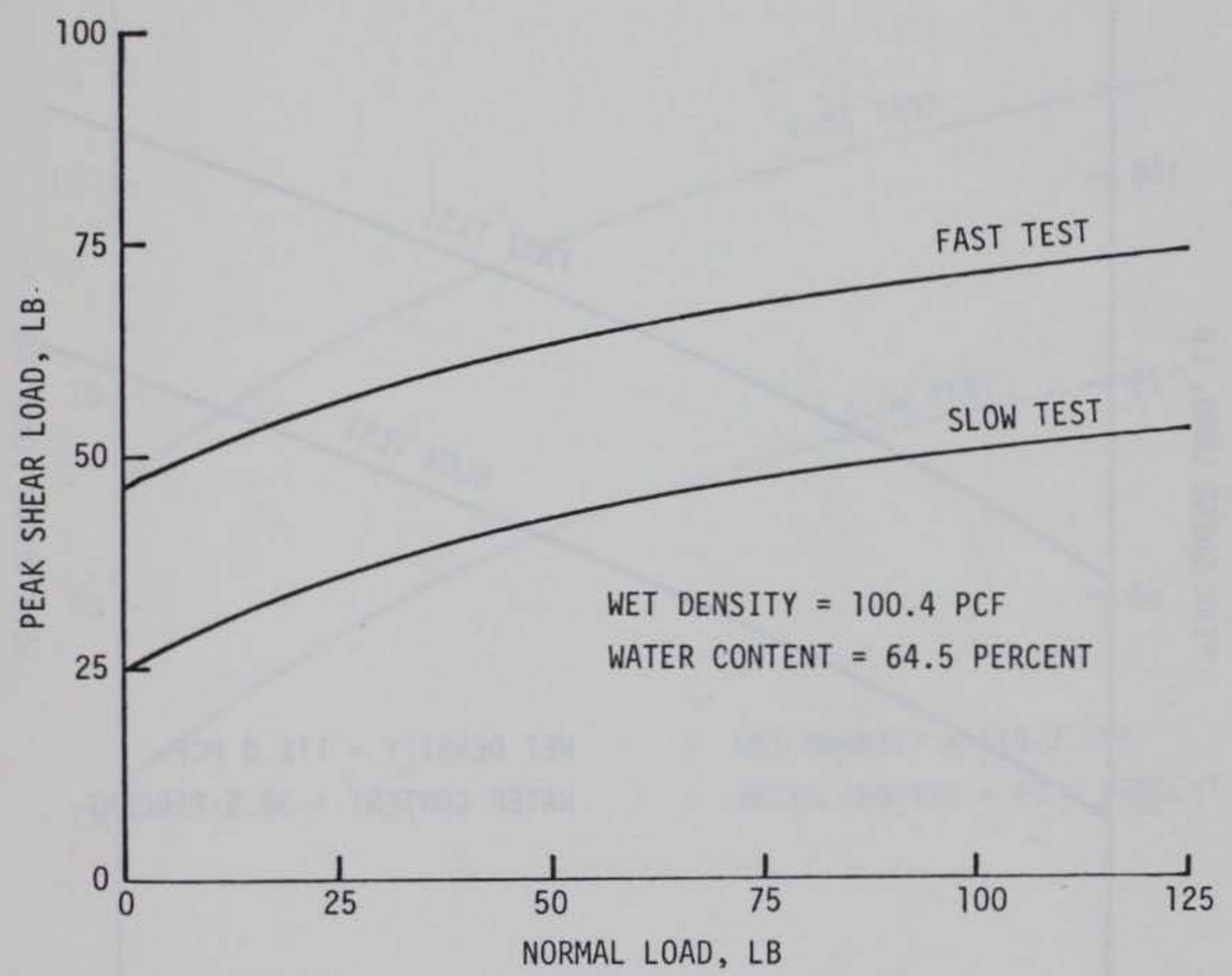

Figure 3. Peak shear load versus normal load (site 7B, test series 107-111) 


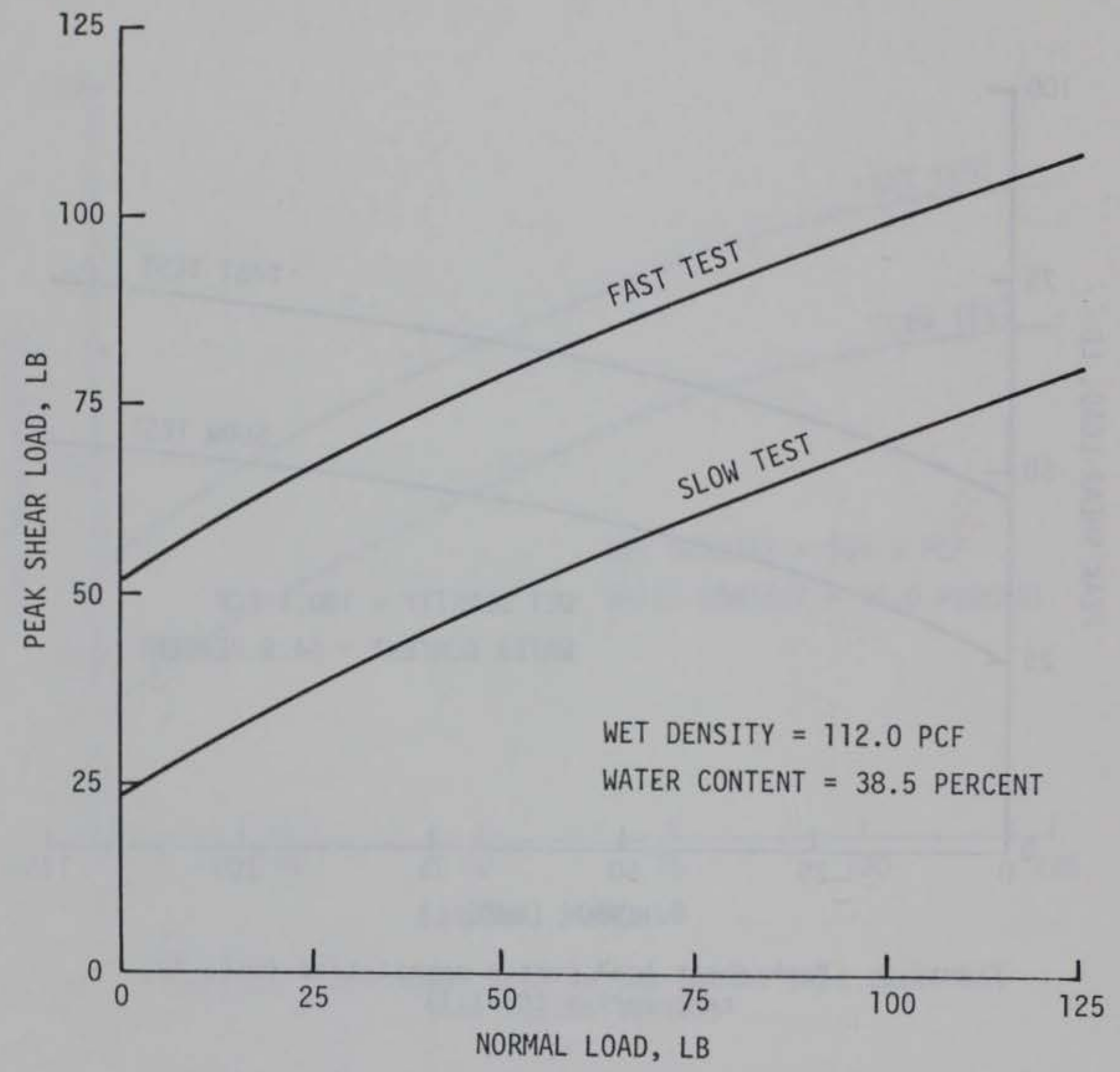

Figure 4. Peak shear load versus normal load (site 7A, test series 117-122) 


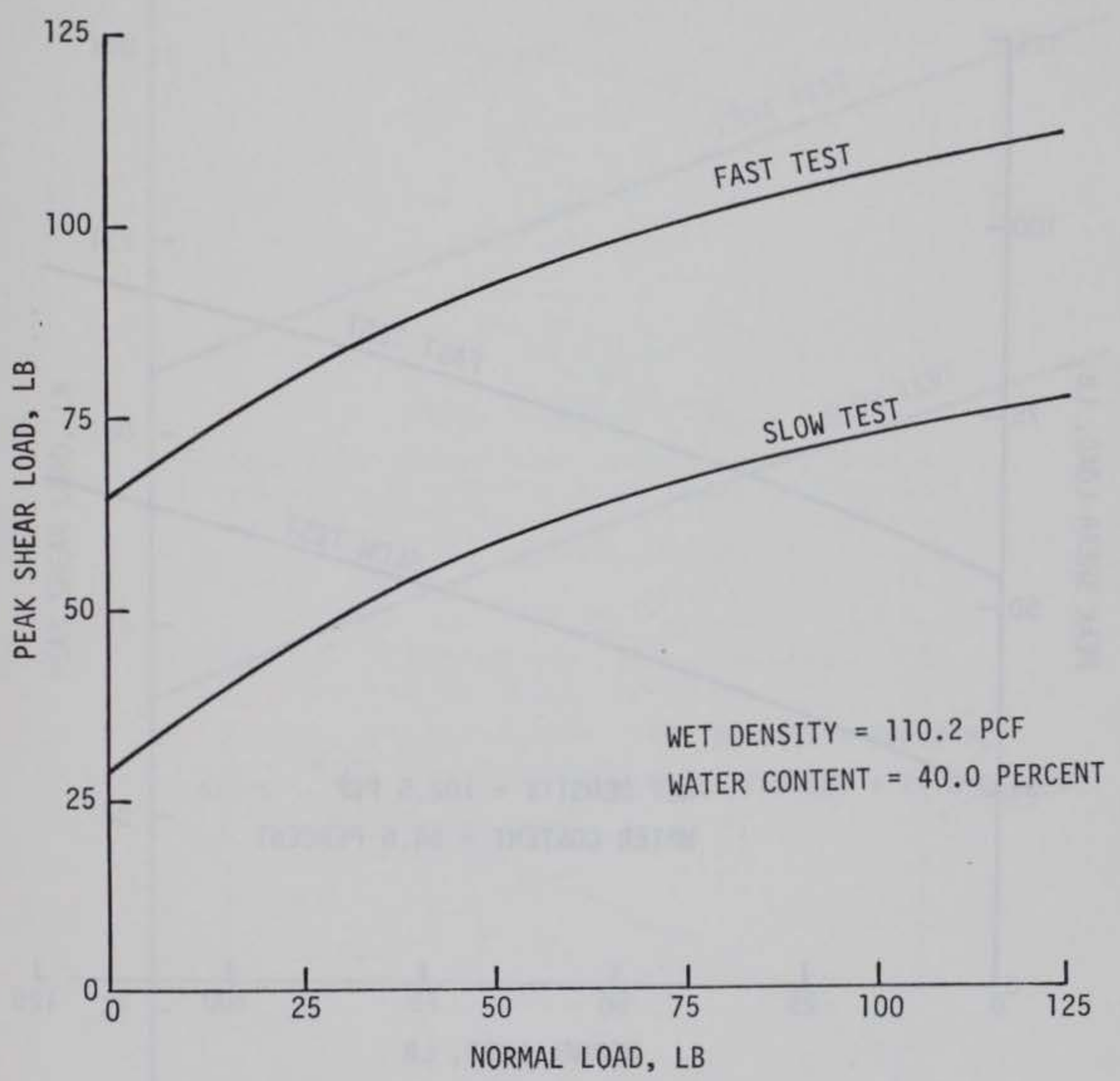

Figure 5. Peak shear load versus normal load (site 7A, test series 131-134) 


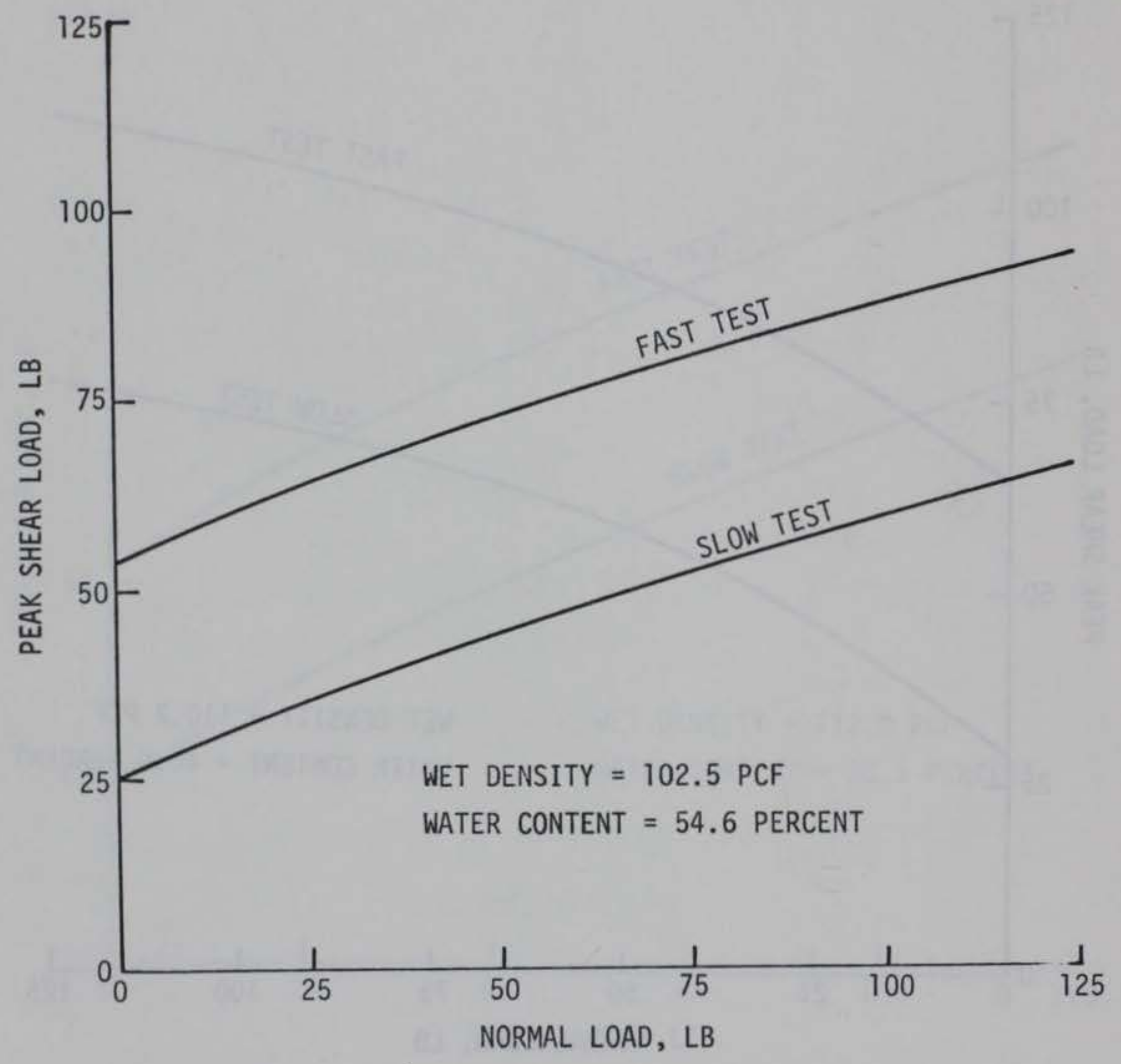

Figure 6. Peak shear load versus normal load (site 7B, test series 139-144) 


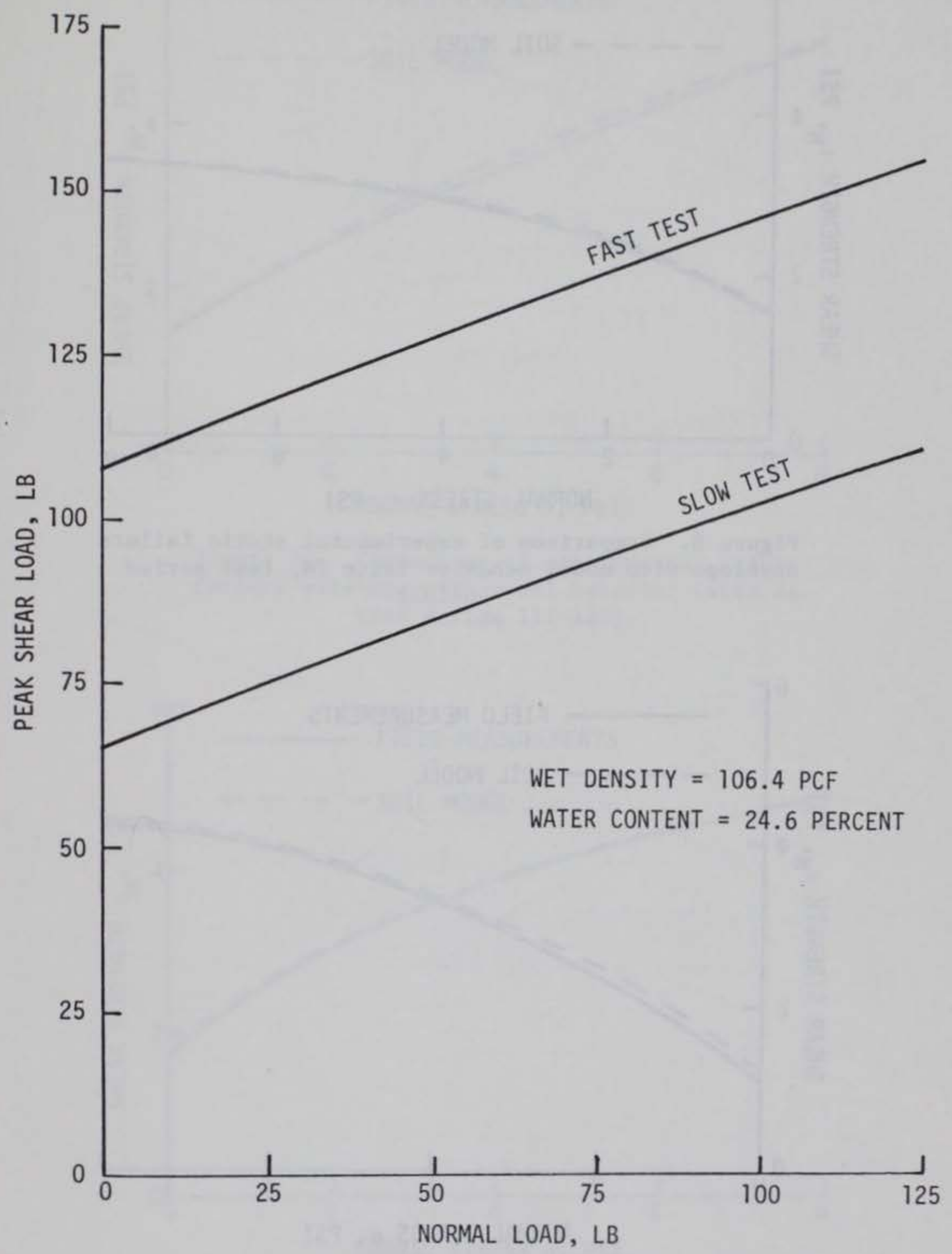

Figure 7. Peak shear load versus normal load (site 8, test series 157-159) 


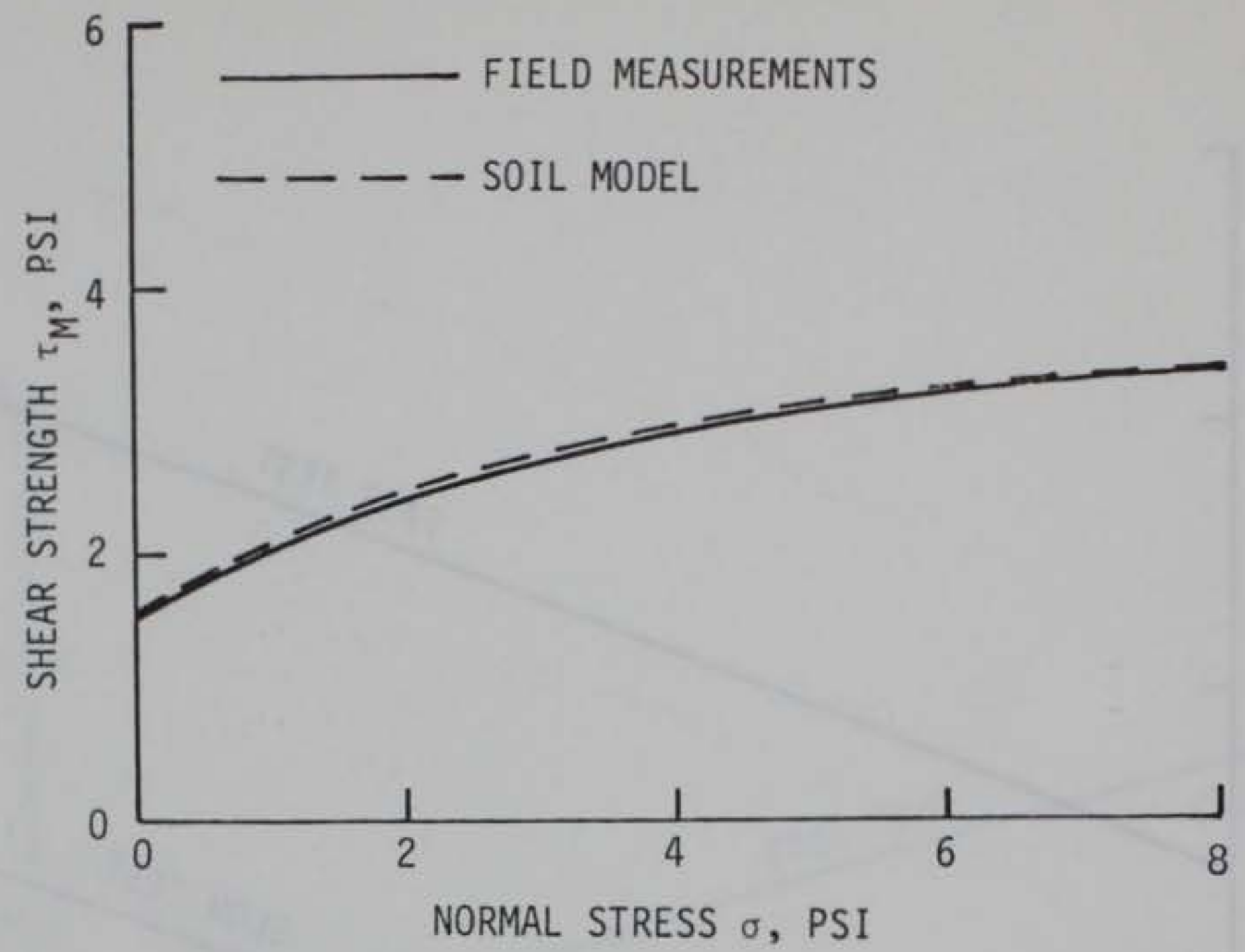

Figure 8. Comparison of experimental static failure envelope with model behavior (site 7A, test series 98-102)

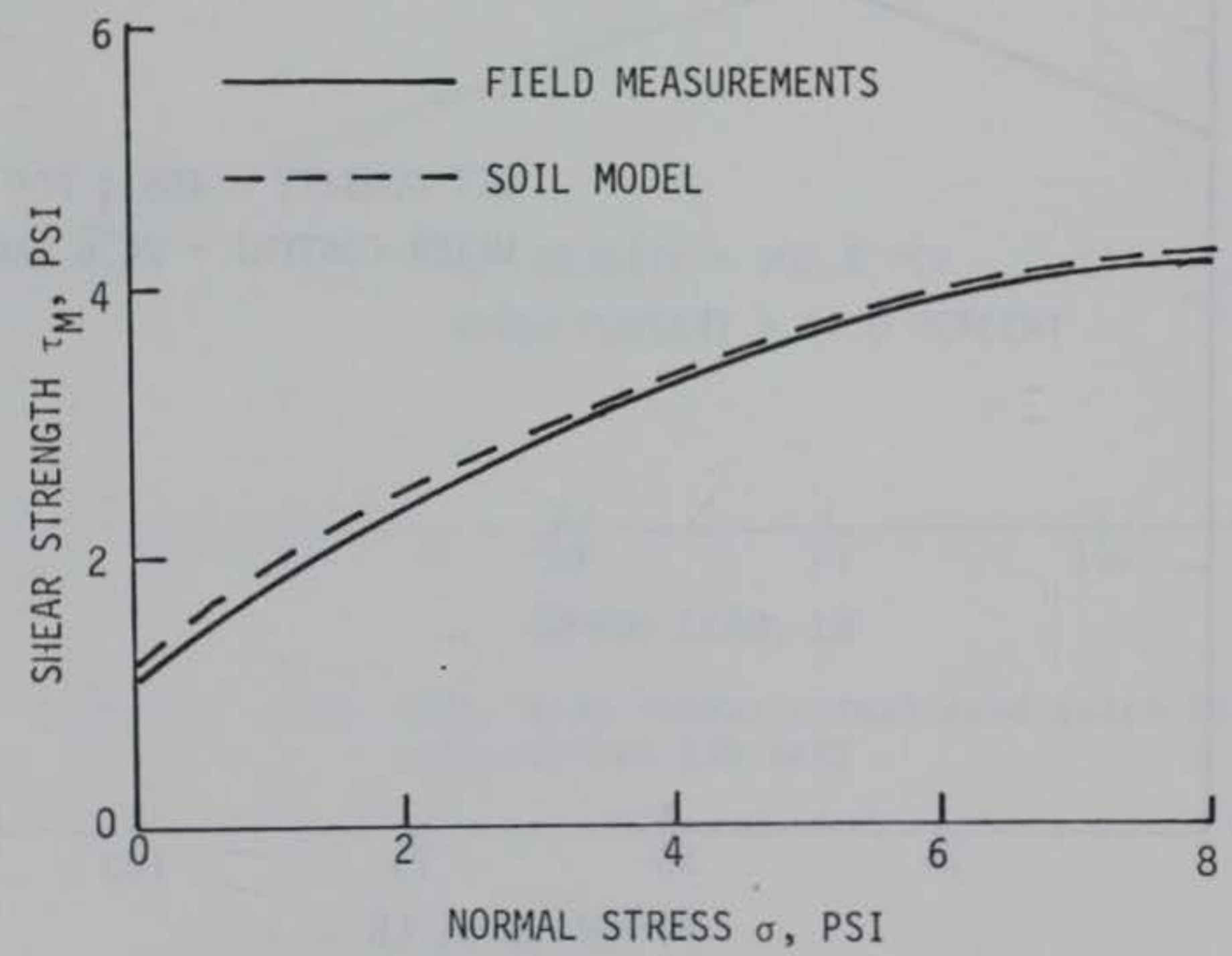

Figure 9. Comparison of experimental static failure envelope with model behavior (site 7B, test series 107-111) 


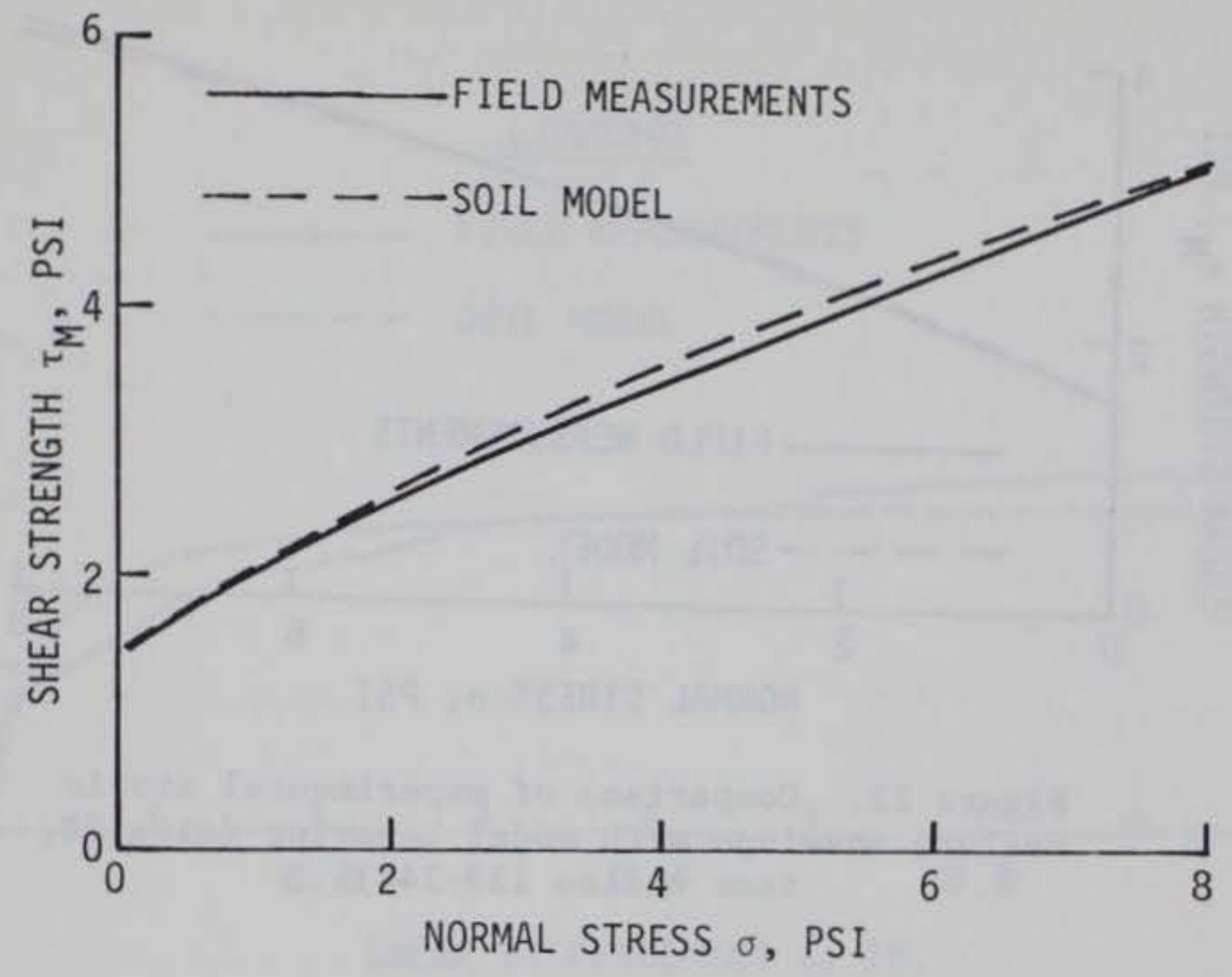

Figure 10. Comparison of experimental static failure envelope with model behavior (site 7A, test series 117-122)

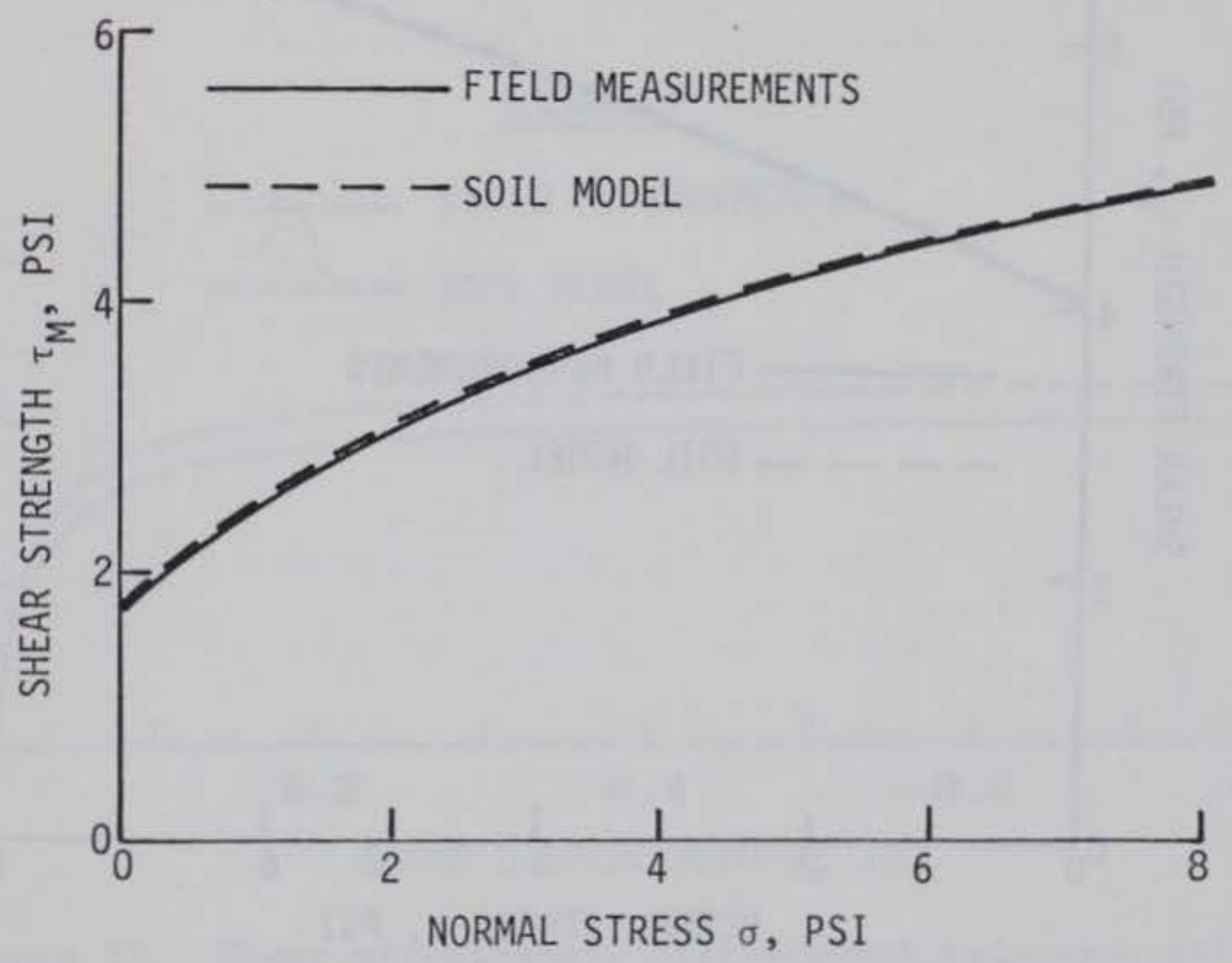

Figure 11. Comparison of experimental static failure envelope with model behavior (site 7A, test series 131-134) 


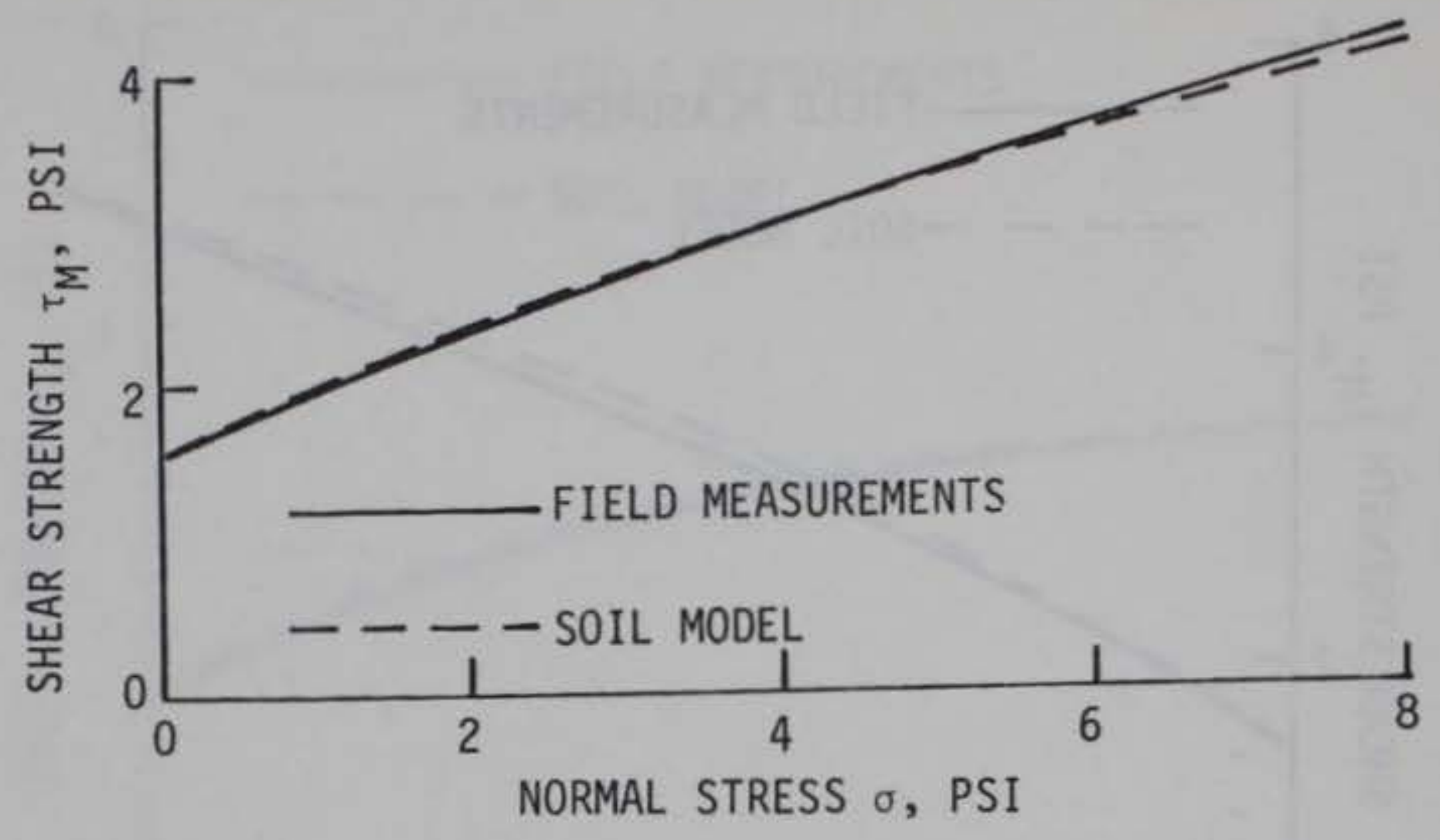

Figure 12. Comparison of experimental static failure envelope with model behavior (site 7B, test series 139-144)

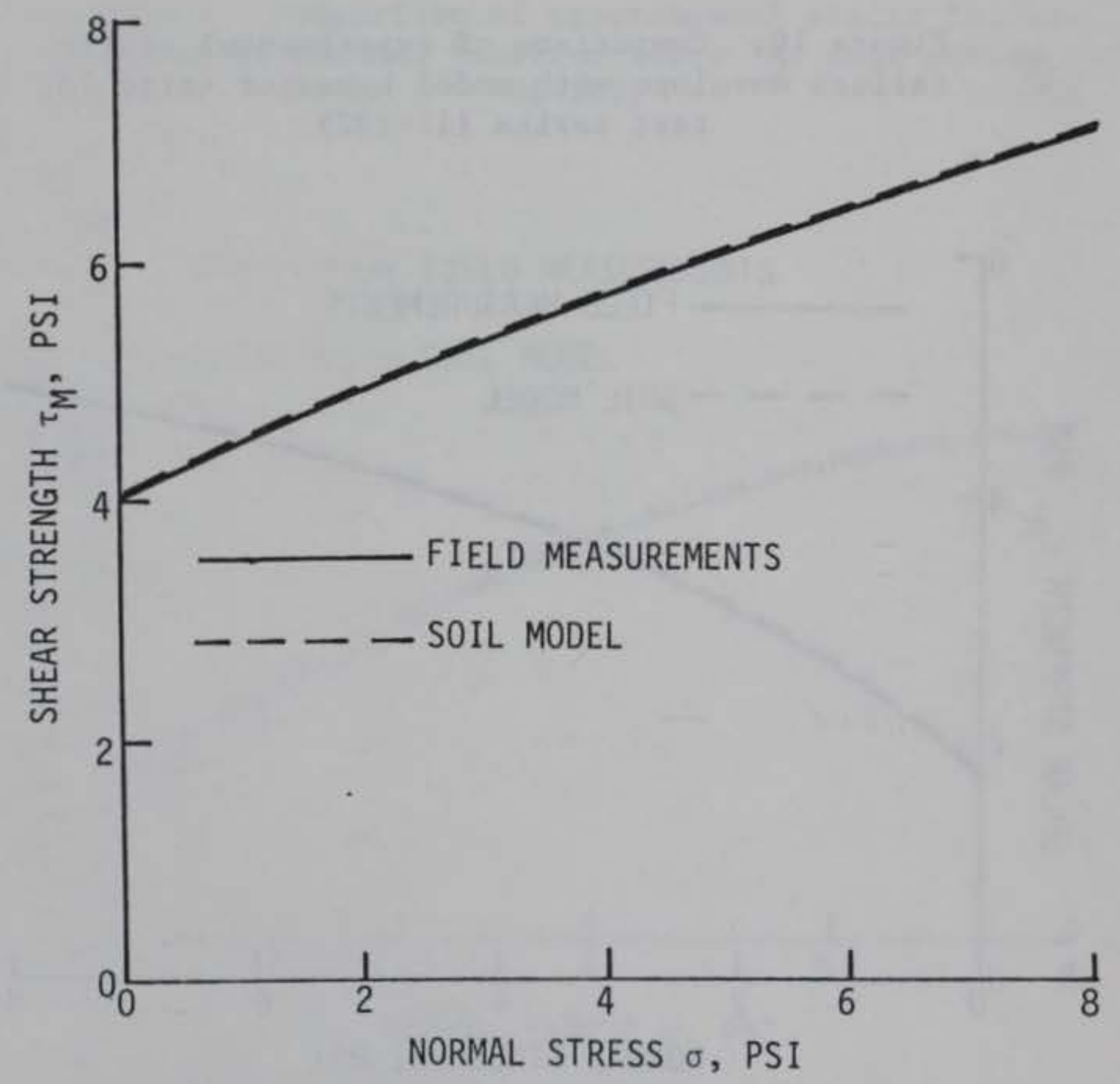

Figure 13. Comparison of experimental static failure envelope with model behavior (site 8 , test series 157-159) 


\section{LEGEND}

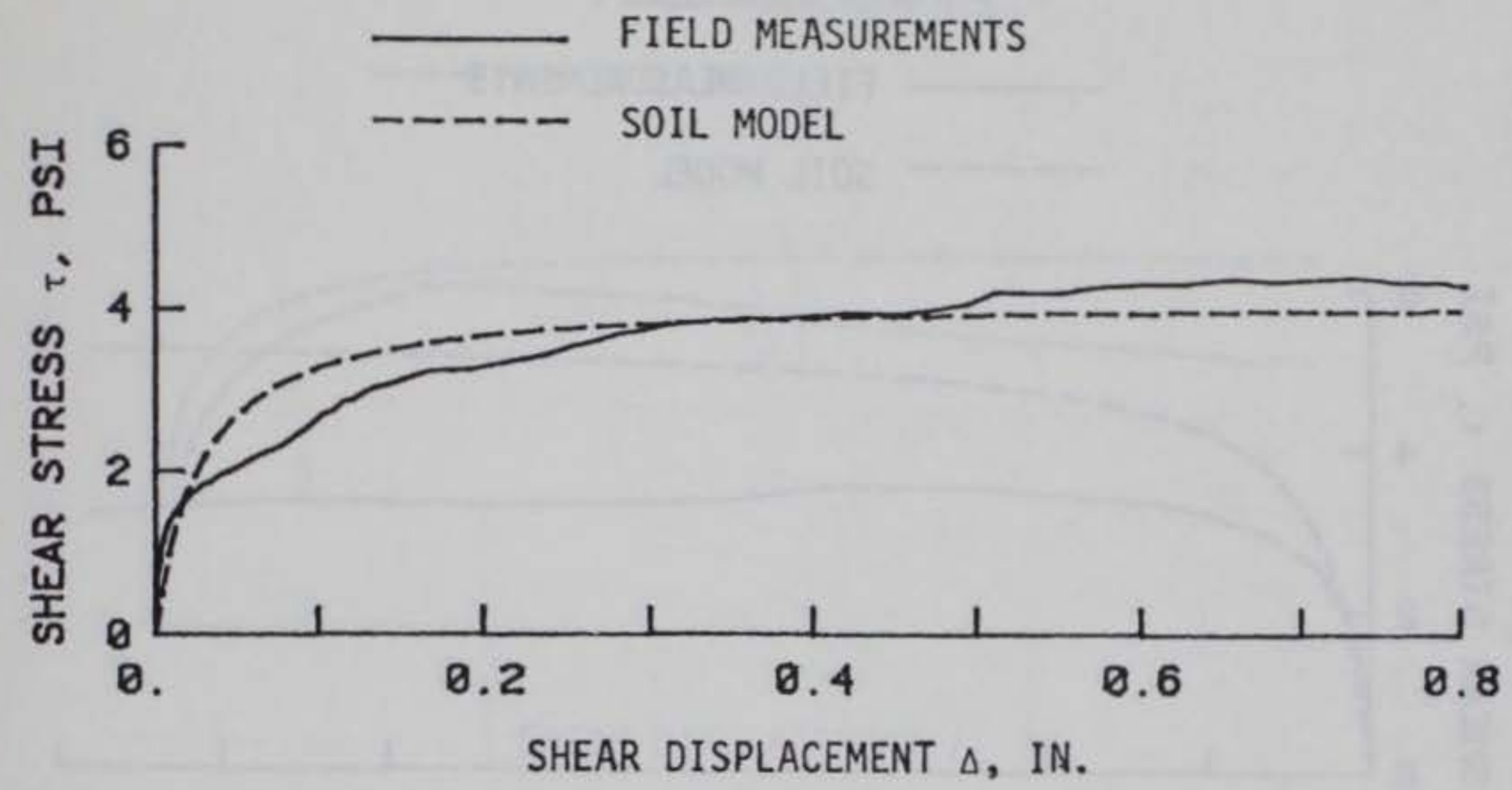

Figure 14. Shear stress-shear displacement relation; field measurements versus model behavior (test series 98-102);

$\sigma=4.11 \mathrm{psi}, \dot{\Delta}=0.75 \mathrm{in} . / \mathrm{sec}$

\section{LEGEND}

\section{FIELD MEASUREMENTS}

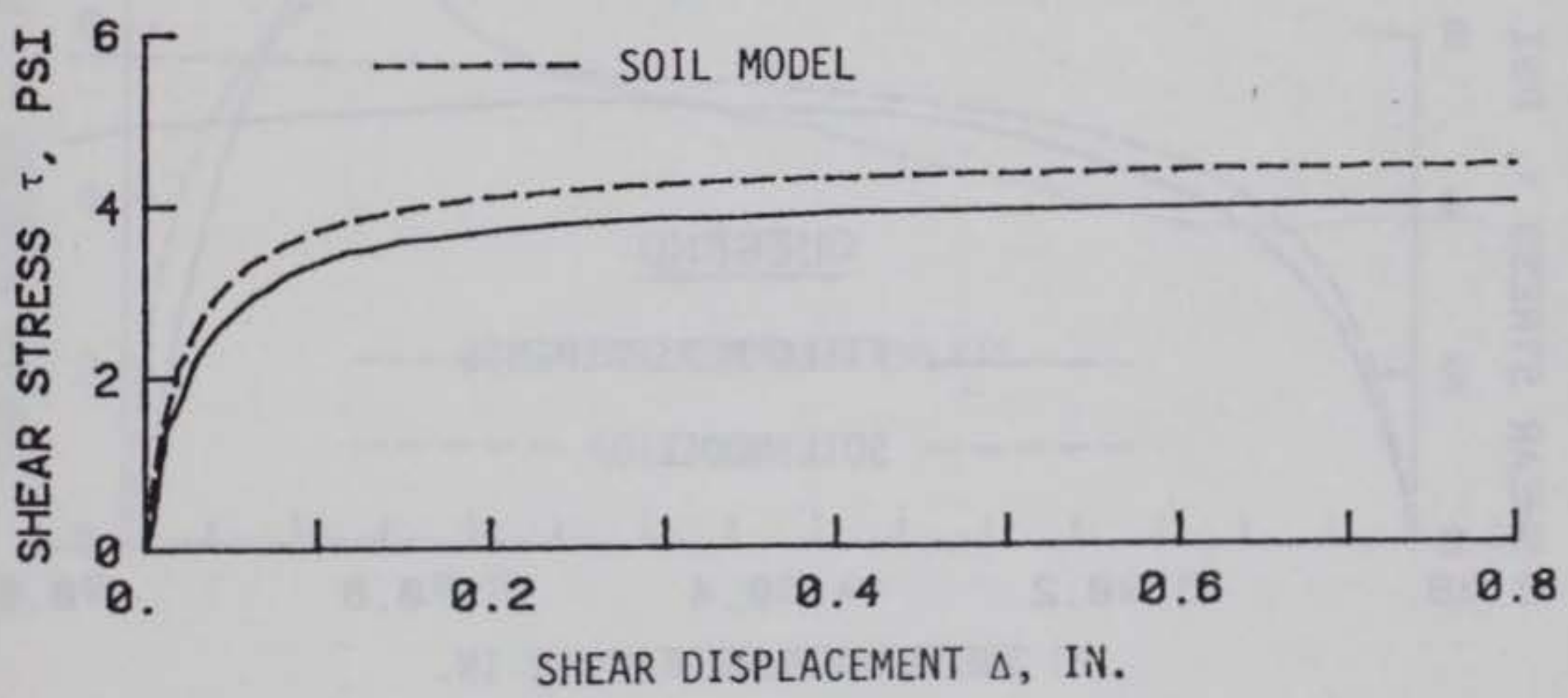

Figure 15. Shear stress-shear displacement relation; field measurements versus model behavior (test series 107-111); $\sigma=4.11 \mathrm{psi}, \dot{\Delta}=0.75 \mathrm{in} . / \mathrm{sec}$ 


\section{LEGEND}

\section{FIELD MEASUREMENTS}

$-\square---$ SOIL MODEL

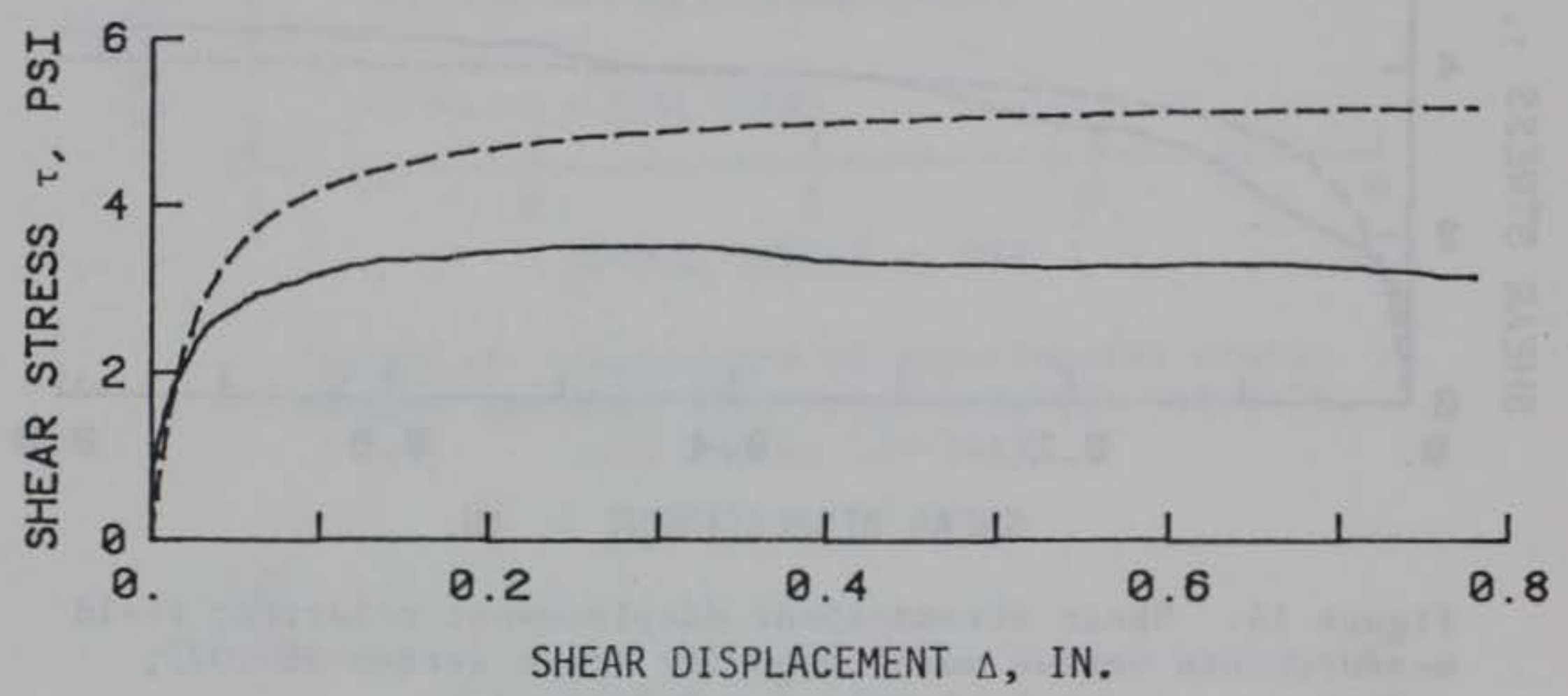

Figure 16. Shear stress-shear displacement relation; field measurements versus model behavior (test series 117-122);

$$
\sigma=4.11 \mathrm{psi}, \dot{\Delta}=0.75 \mathrm{in} . / \mathrm{sec}
$$

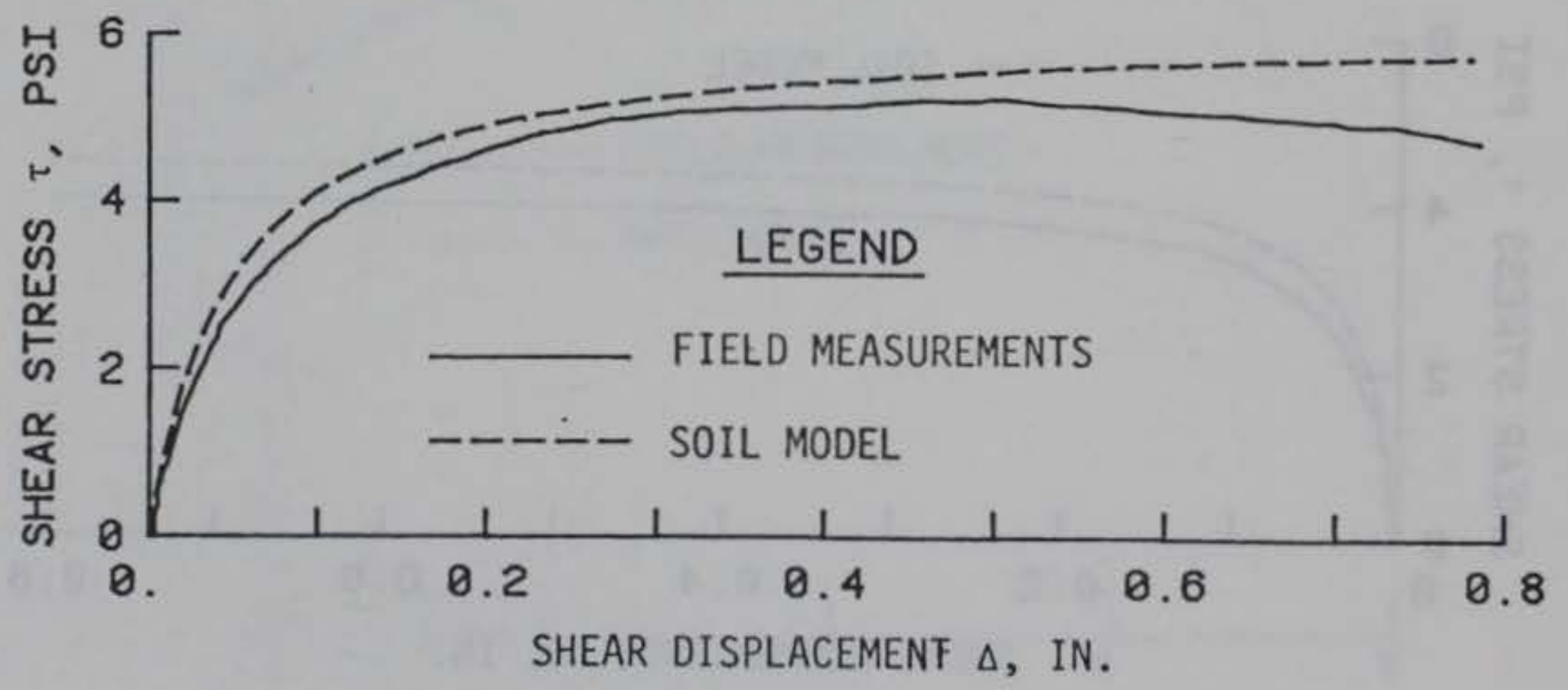

Figure 17. Shear stress-shear displacement relation; field measurements versus model behavior (test series 131-134);

$$
\sigma=4.11 \mathrm{psi}, \dot{\Delta}=0.75 \mathrm{in} . / \mathrm{sec}
$$




\section{LEGEND}

\section{FIELD MEASUREMENTS}

-ー-ー SOIL MODEL

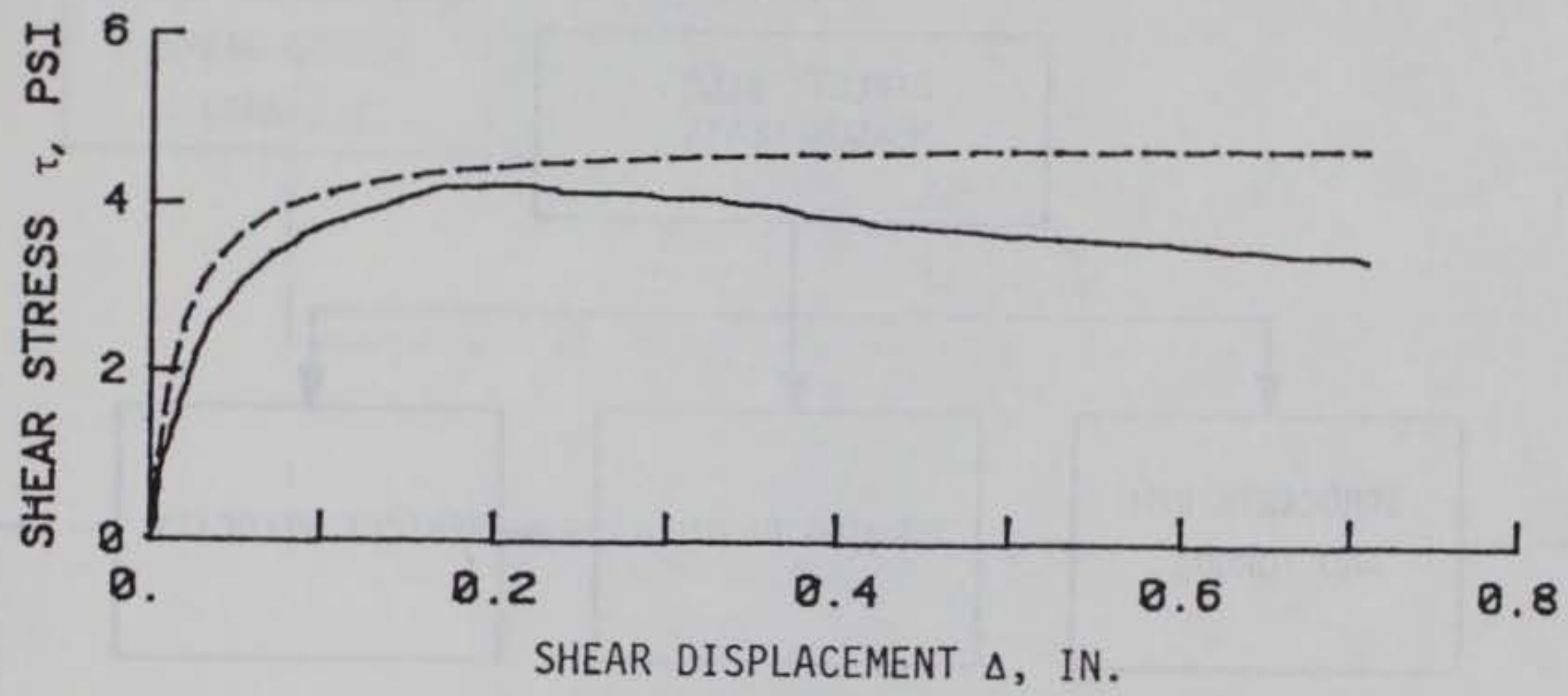

Figure 18. Shear stress-shear displacement relation; field measurements versus model behavior (test series 139-144); $\sigma=4.11 \mathrm{psi}, \dot{\Delta}=0.75 \mathrm{in} . / \mathrm{sec}$

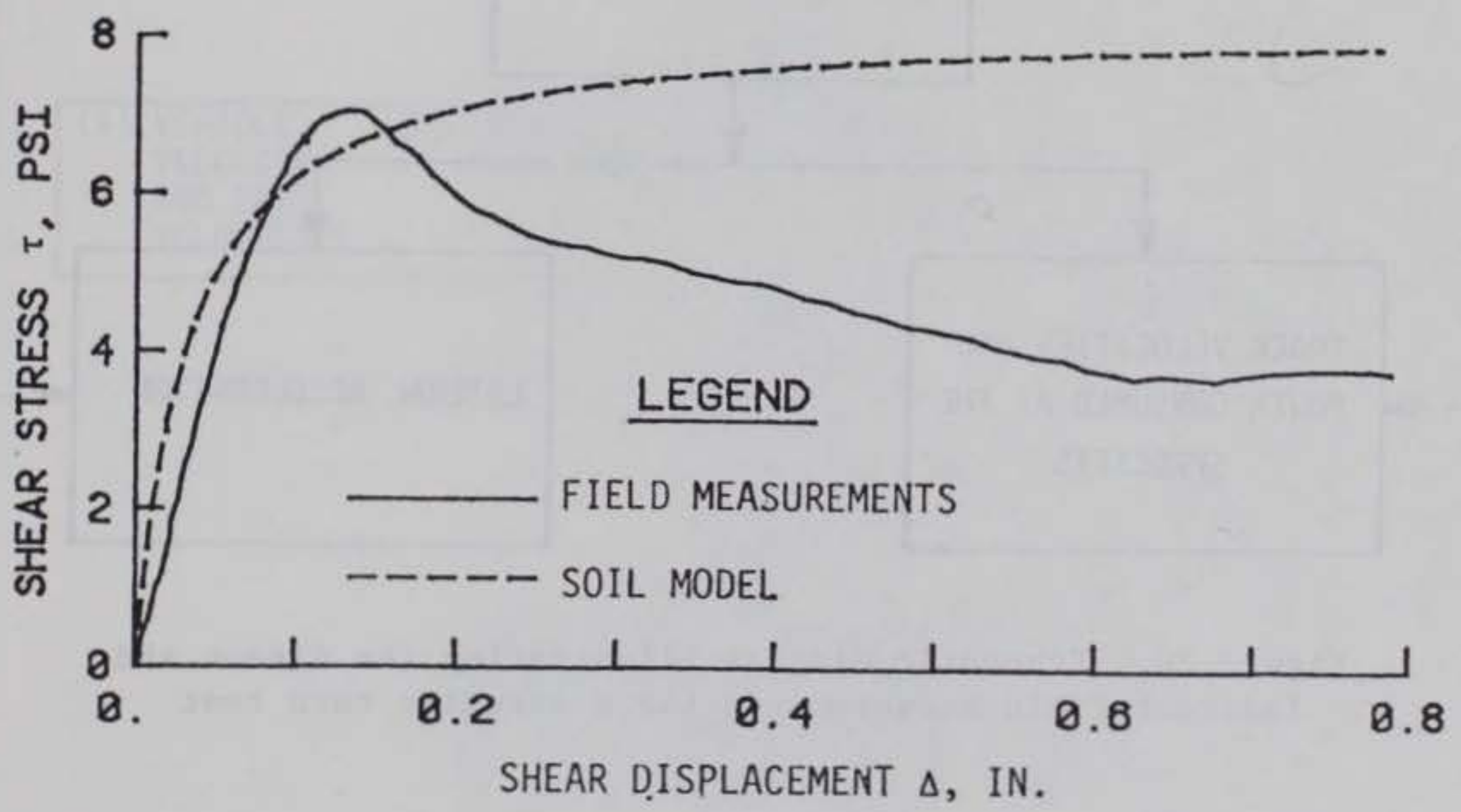

Figure 19. Shear stress-shear displacement relation; field measurements versus model behavior (test series 157-159); $\sigma=4.11 \mathrm{psi}, \dot{\Delta}=0.75 \mathrm{in} . / \mathrm{sec}$ 


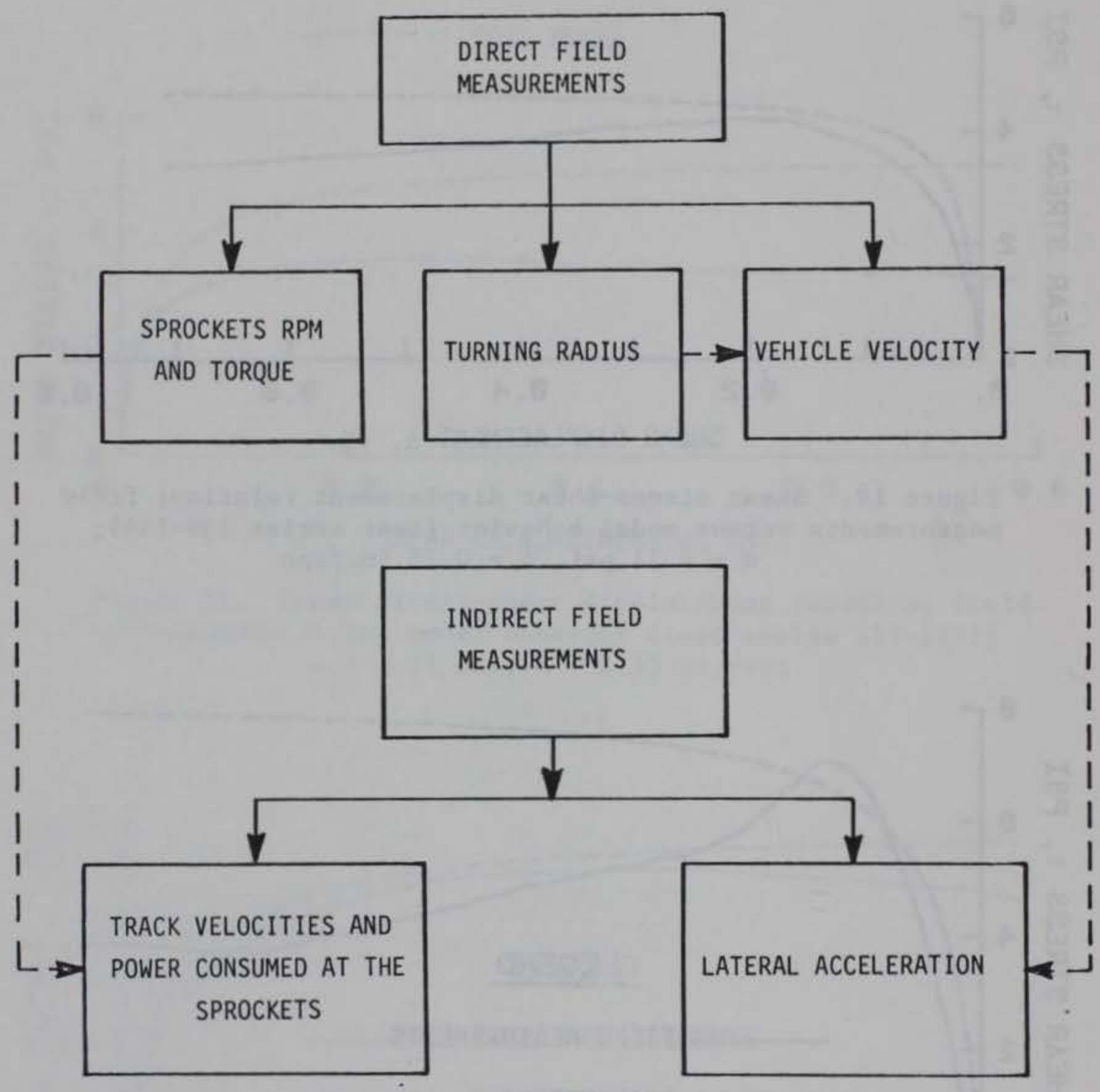

Figure 20. Schematic diagram illustrating the direct and indirect field measurements for a circular turn test 


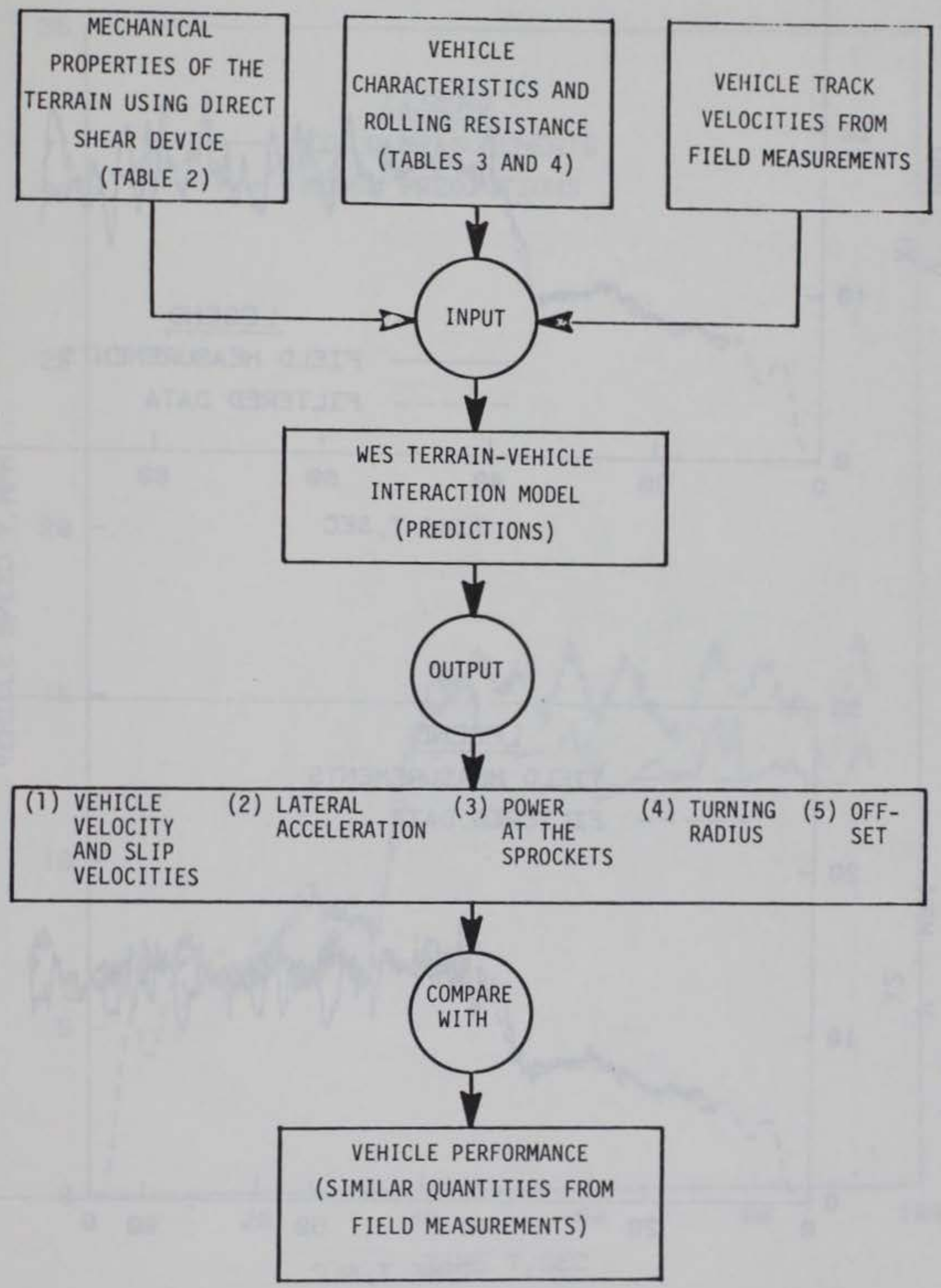

Figure 21. Schematic diagram illustrating the validation procedure for the WES terrain-vehicle interaction model 

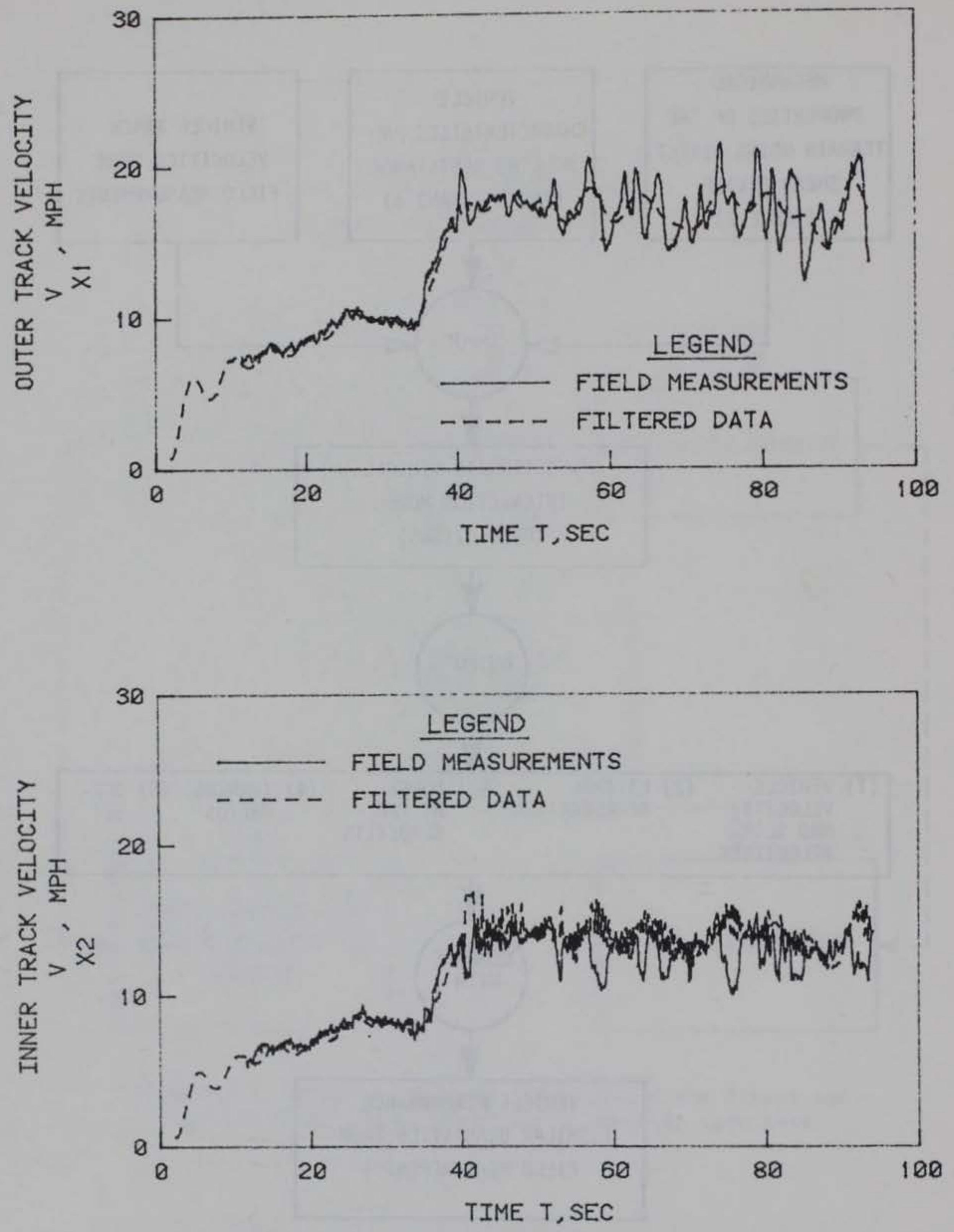

Figure 22. Outer and inner track velocity-time histories for test 99; field measurement and filtered data (input) 


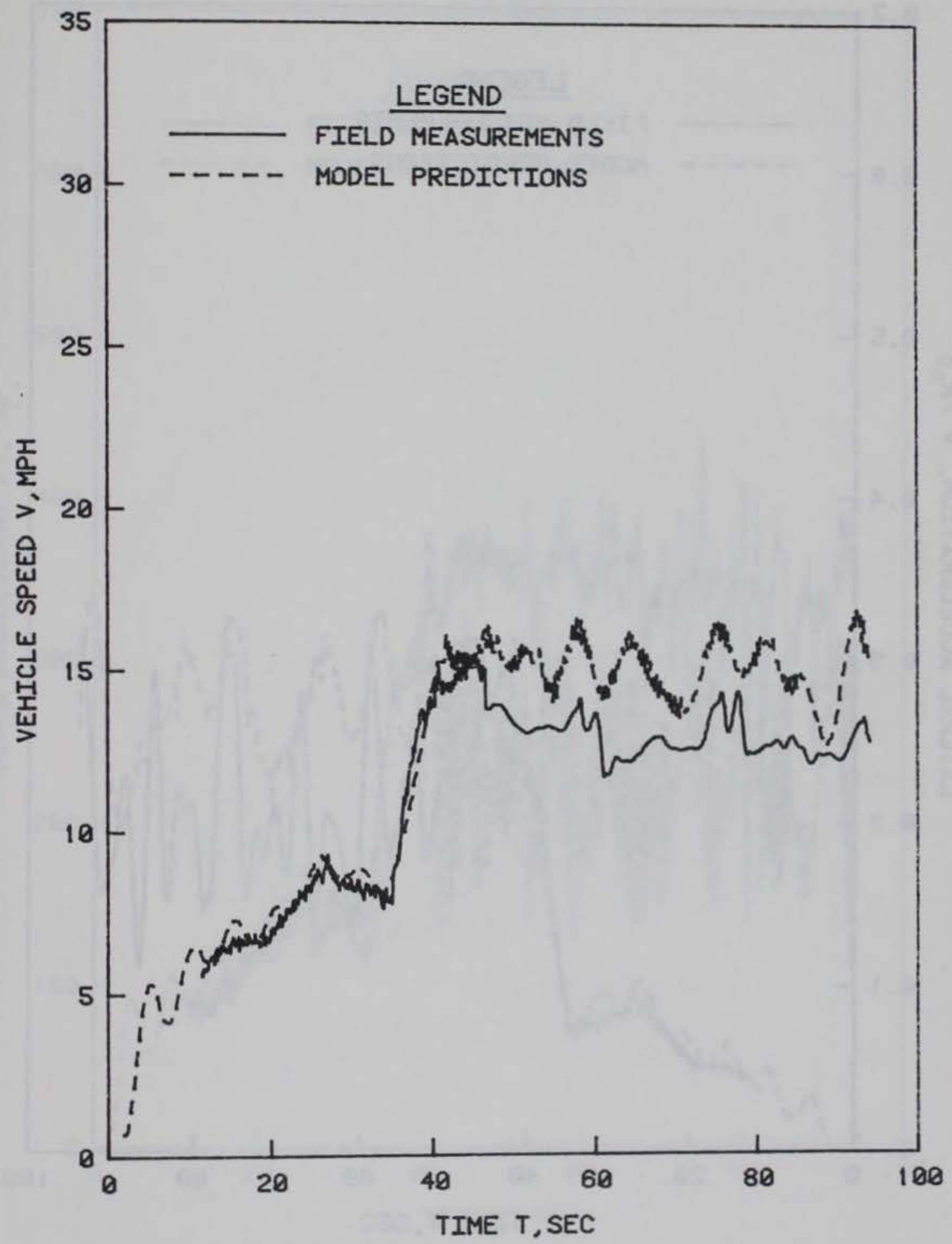

Figure 23. Vehicle speed-time history for test 99; comparison of model predictions with experimental data 


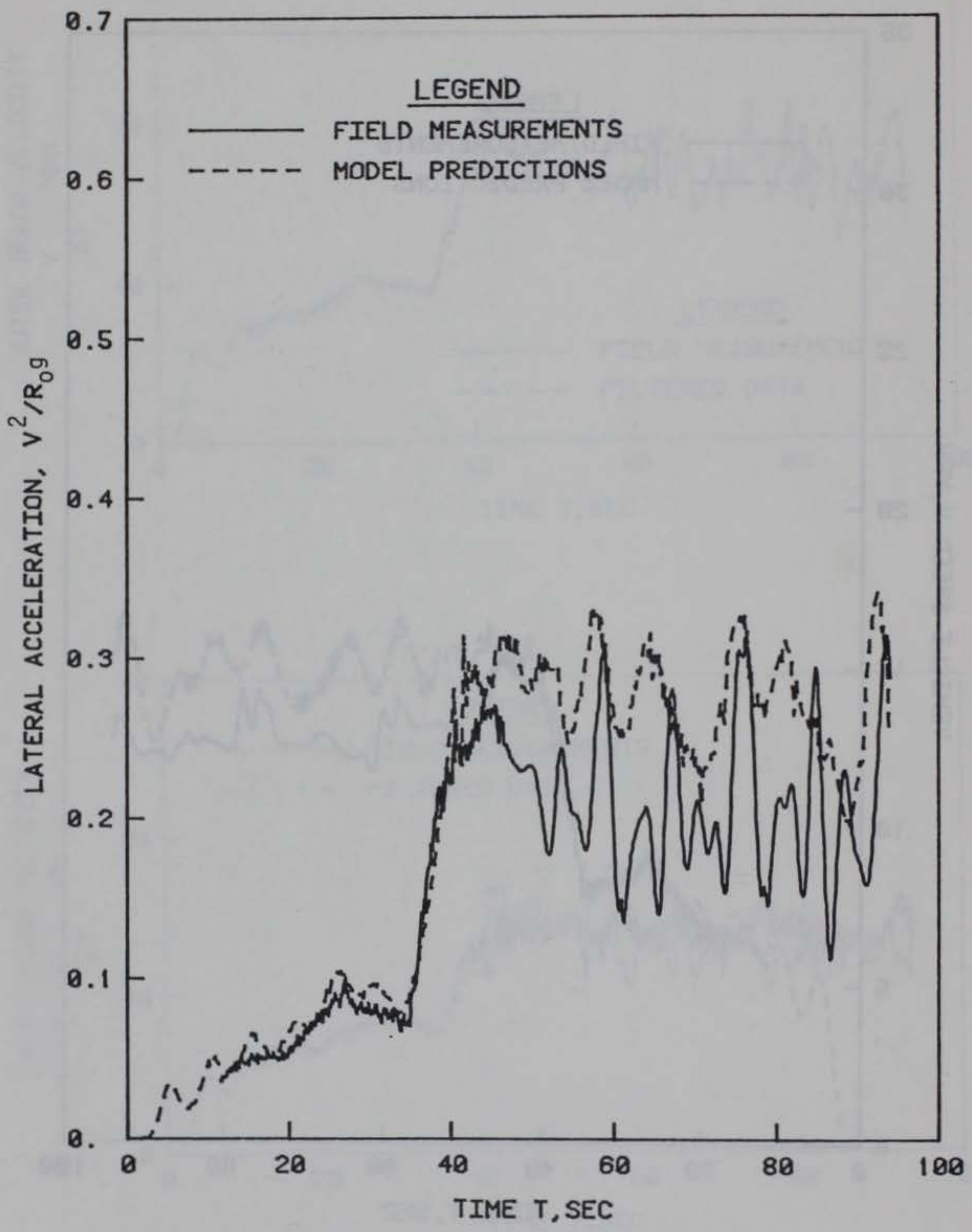

Figure 24. Lateral acceleration-time history for test 99; comparison of model predictions with experimental data 


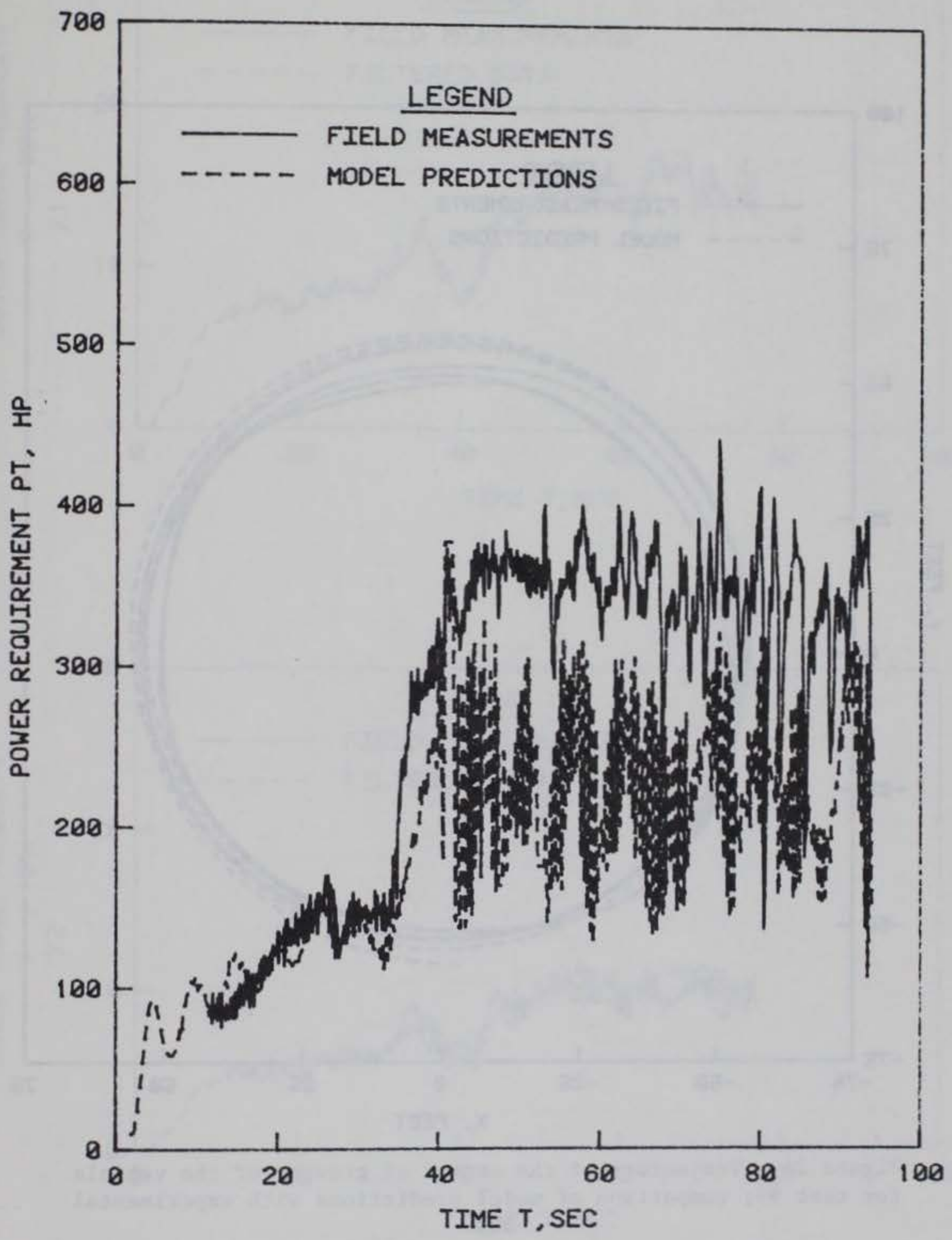

Figure 25. Total power-time history for test 99; comparison of model predictions with experimental data 


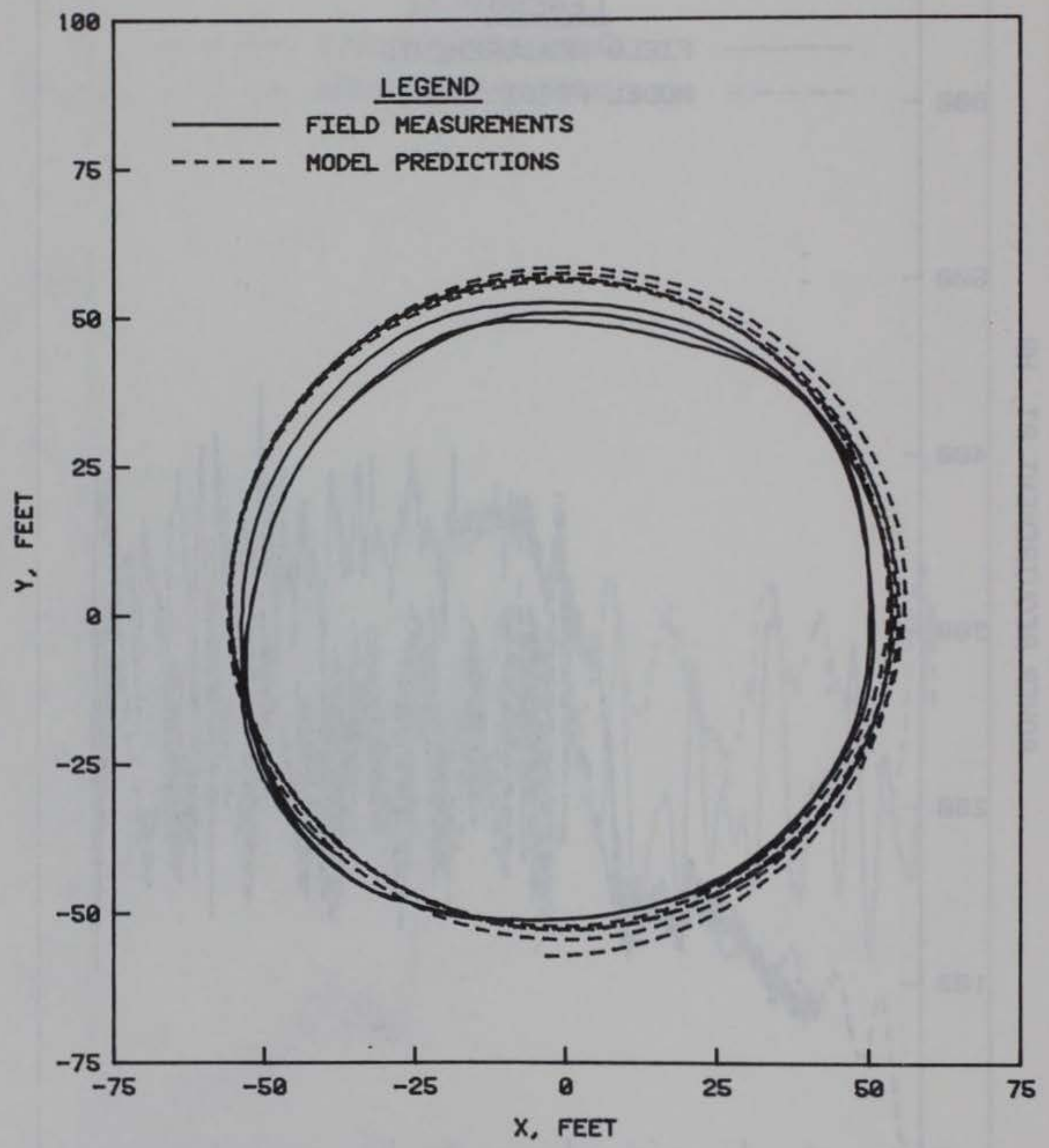

Figure 26. Trajectory of the center of gravity of the vehicle for test 99; comparison of model predictions with experimental data 

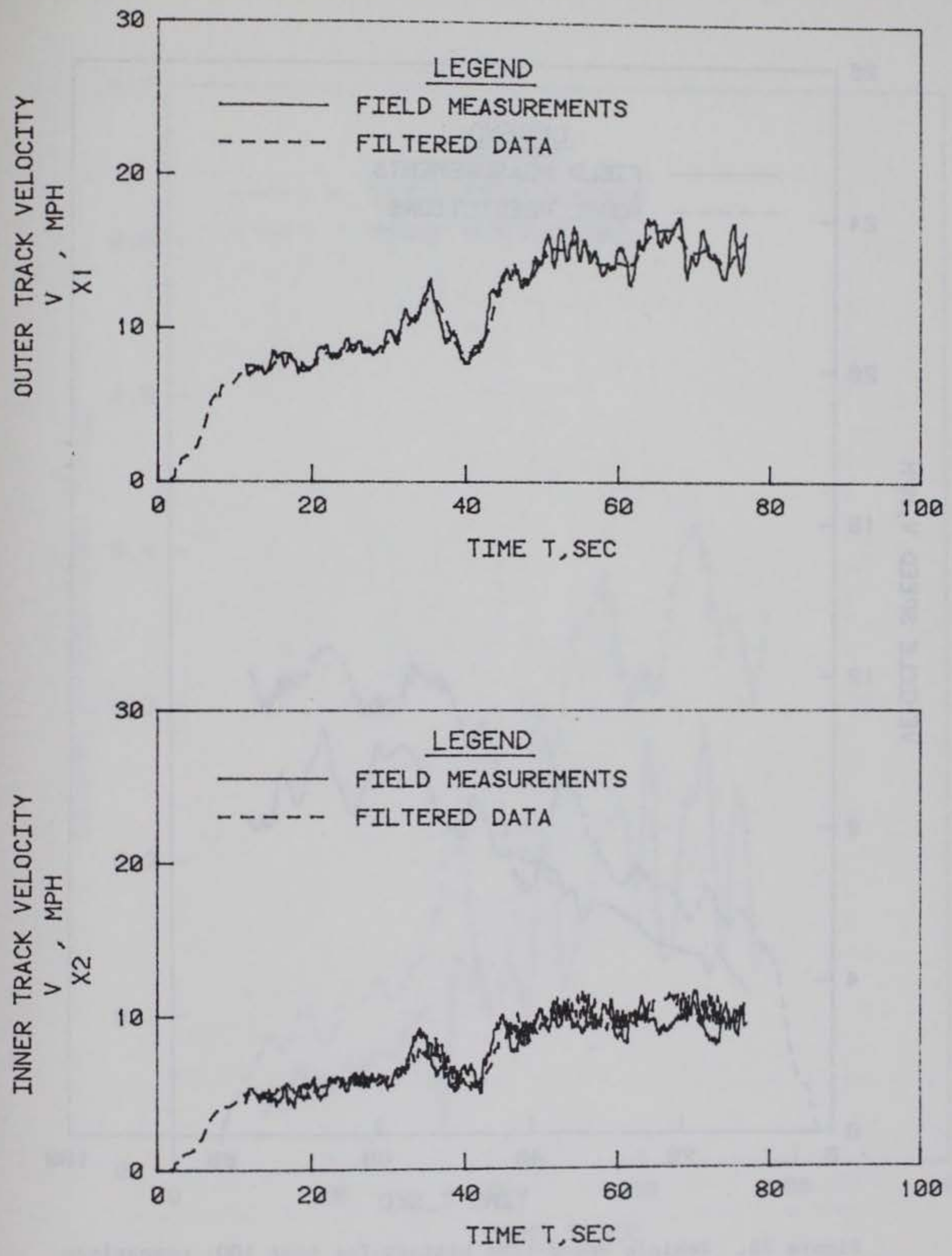

Figure 27. Outer and inner track velocity-time histories for test 100; field measurement and filtered data (input) 


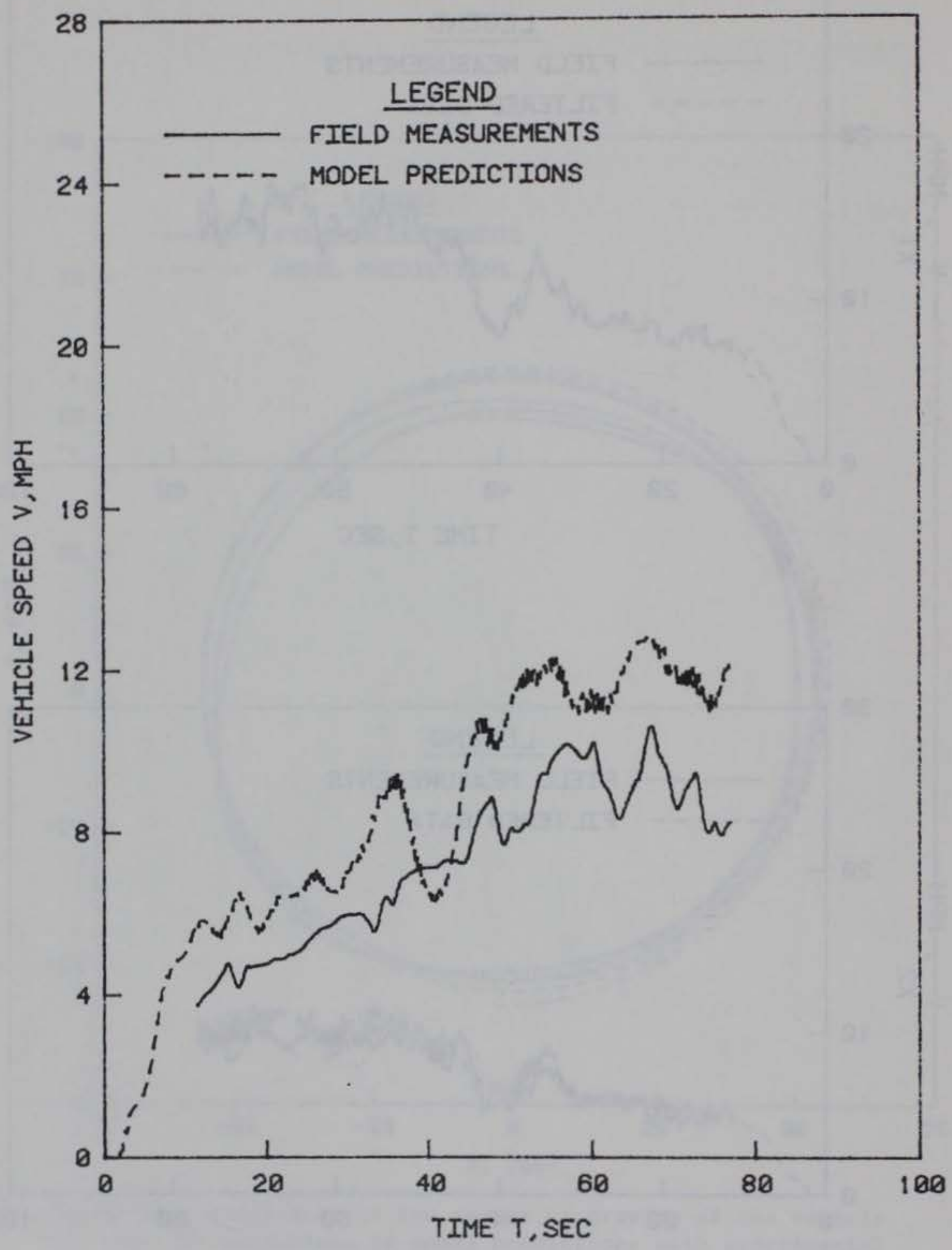

Figure 28. Vehicle speed-time history for test 100; comparison of model predictions with experimental data 


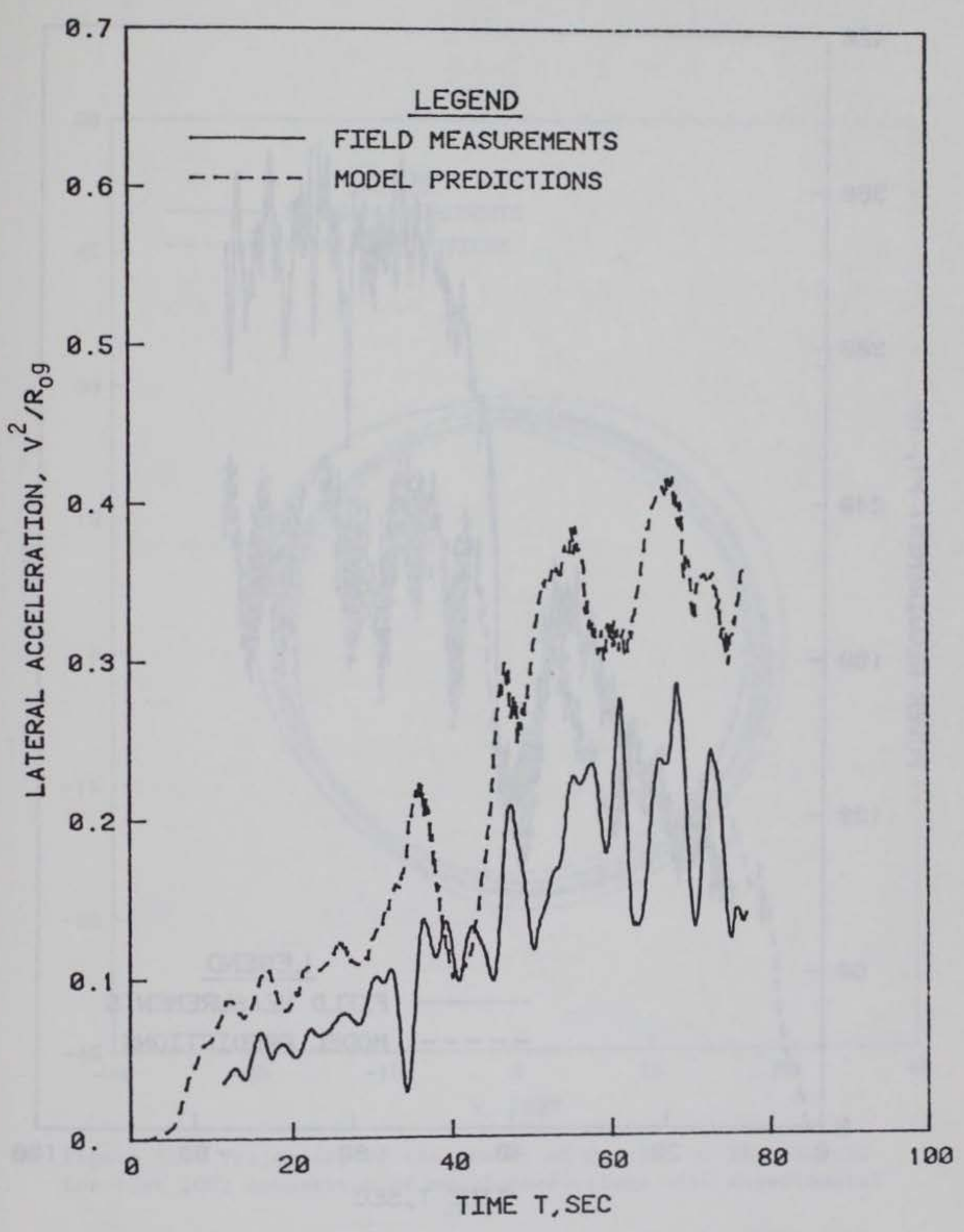

Figure 29. Lateral acceleration-time history for test 100; comparison of model predictions with experimental data 


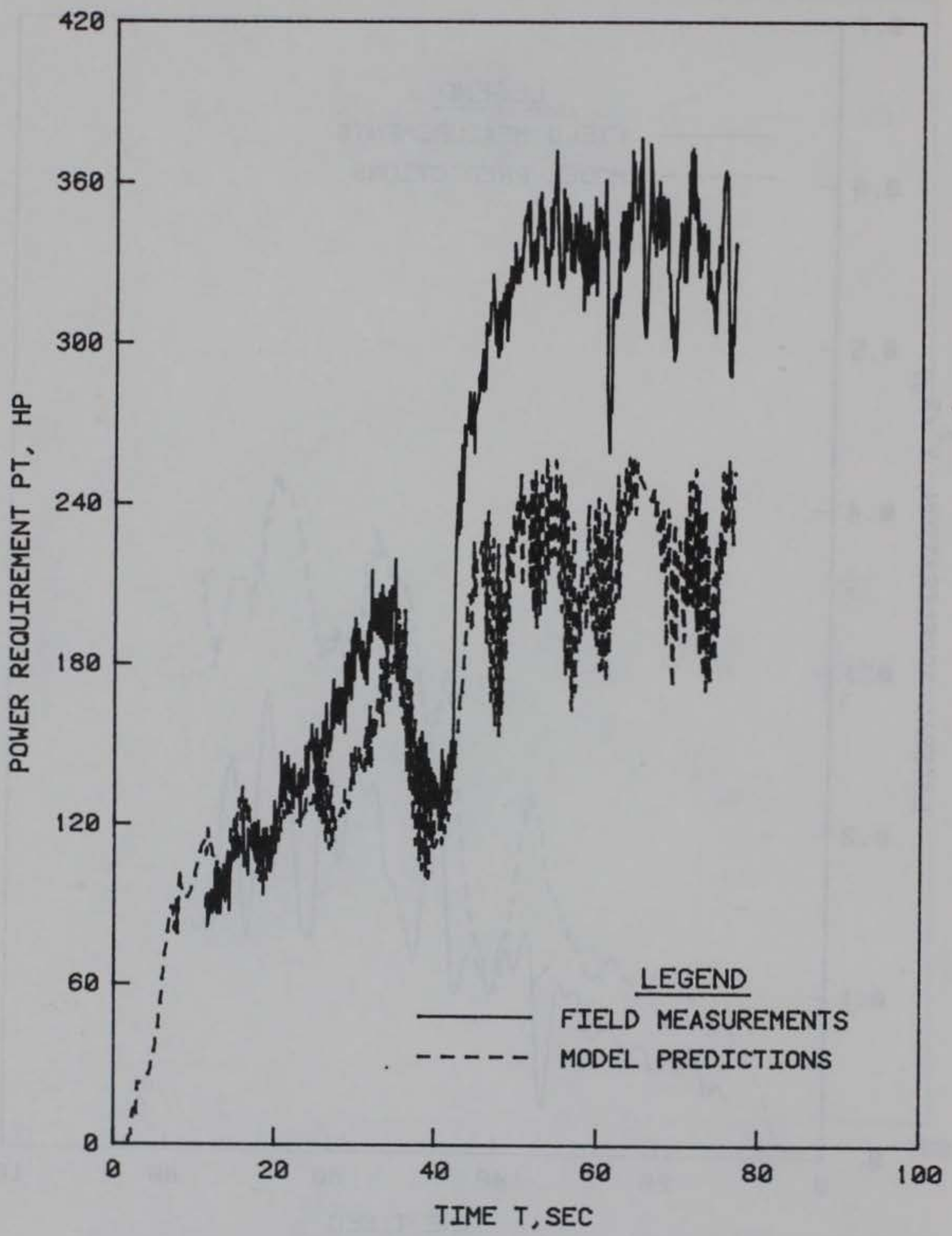

Figure 30. Total power-time history for test 100; comparison of model predictions with experimental data 


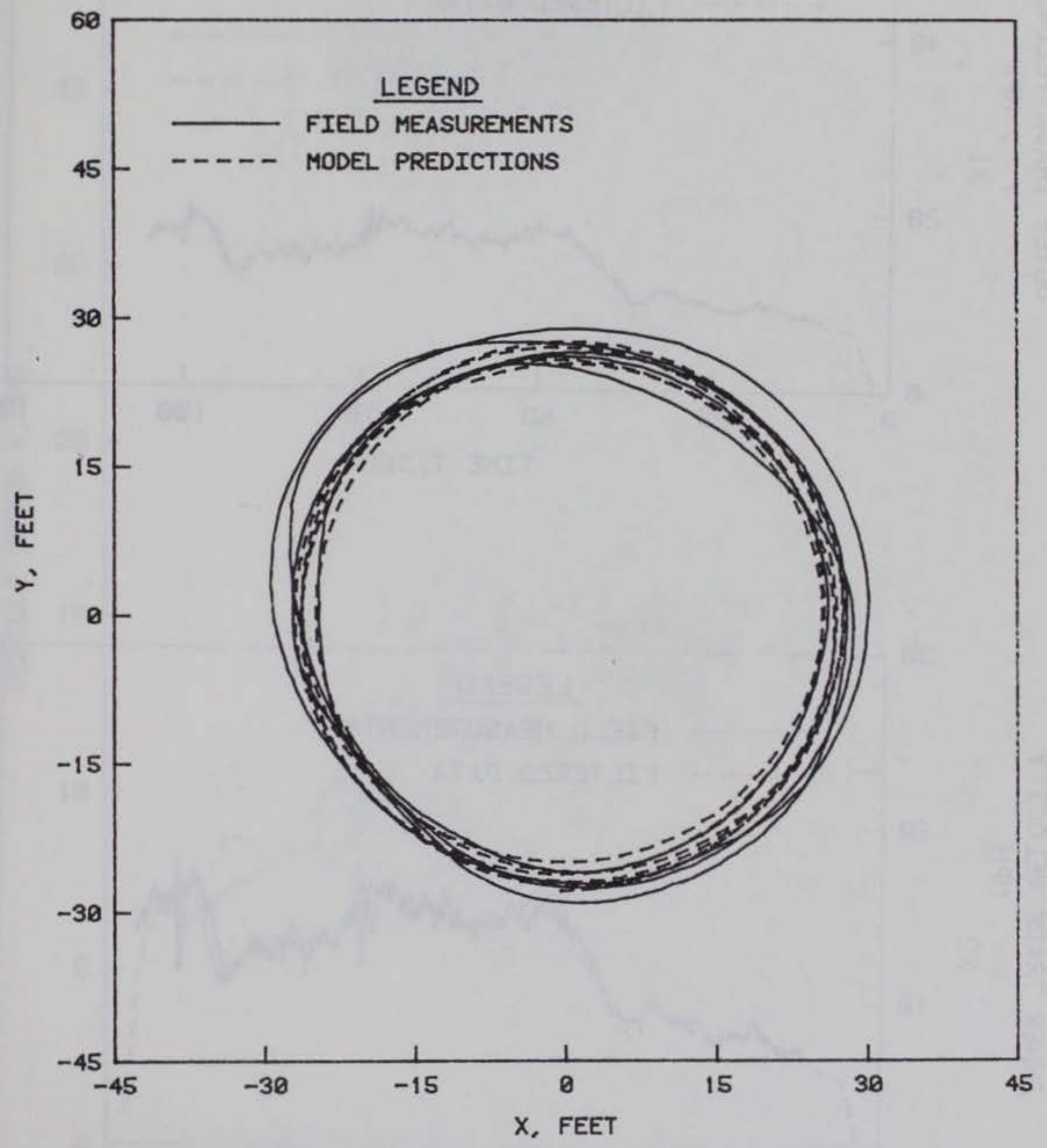

Figure 31. Trajectory of the center of gravity of the vehicle for test 100; comparison of model predictions with experimental data 

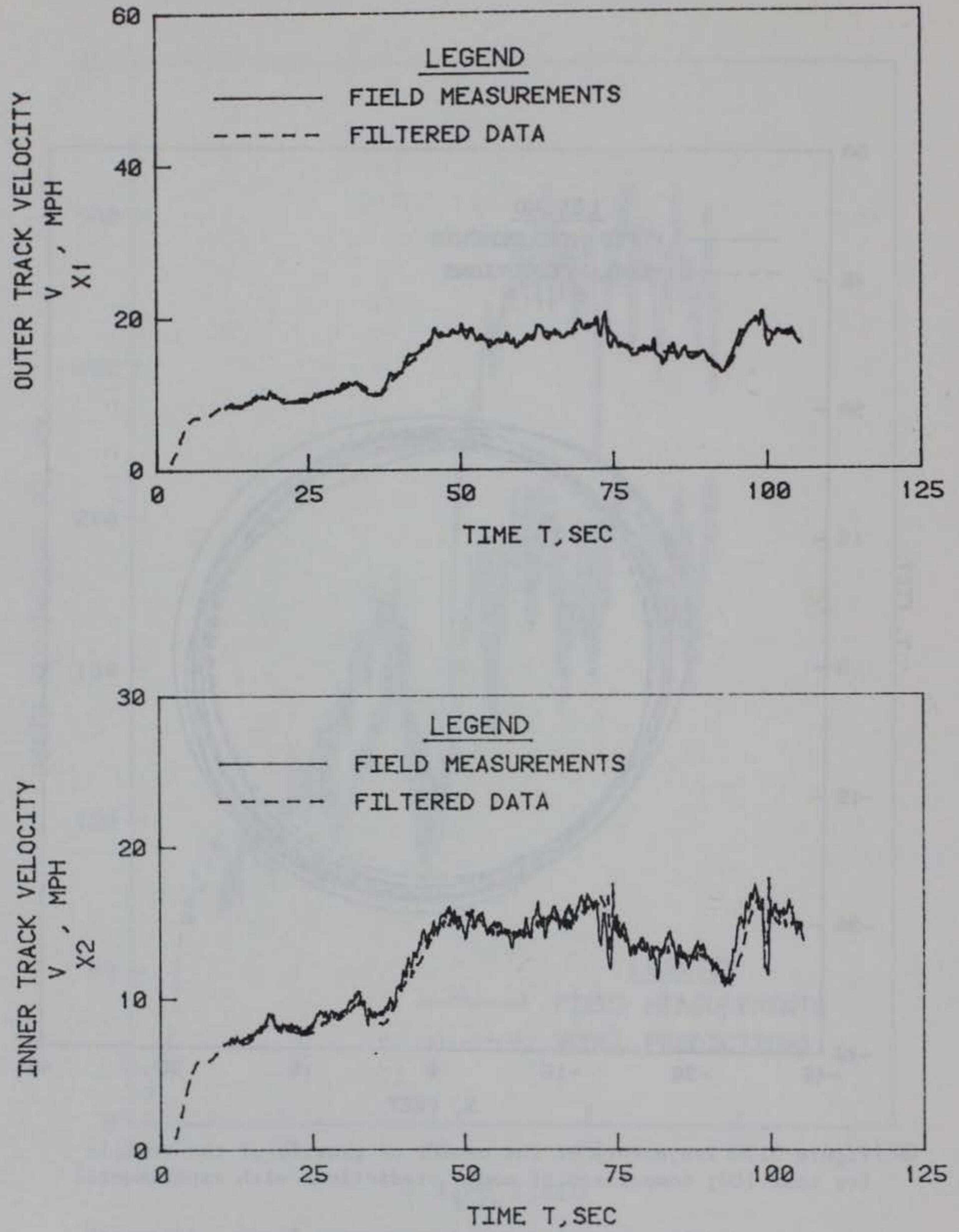

Figure 32. Outer and inner track velocity-time histories for test 101; field measurement and filtered data (input) 


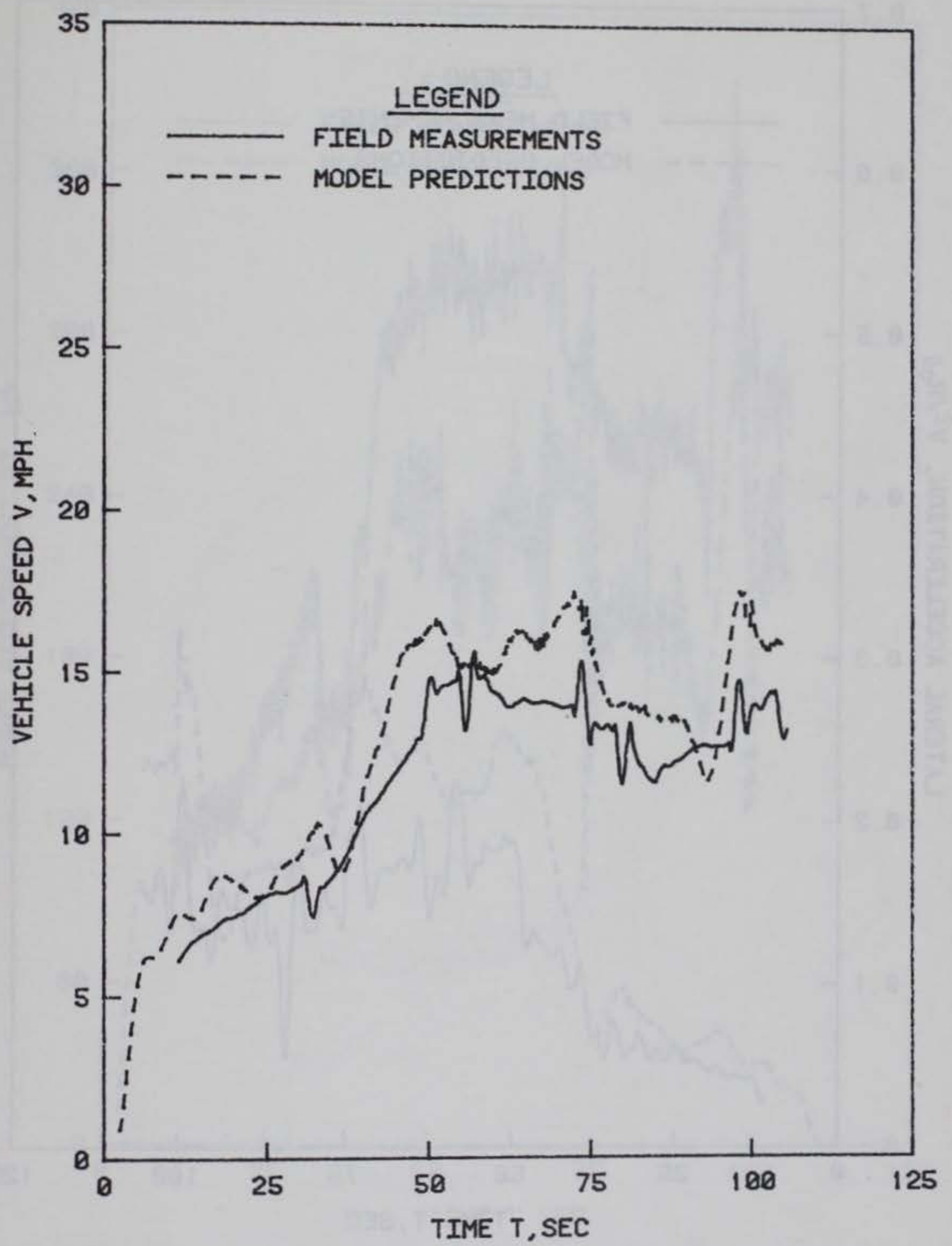

Figure 33. Vehicle speed-time history for test 101; comparison of model predictions with experimental data 


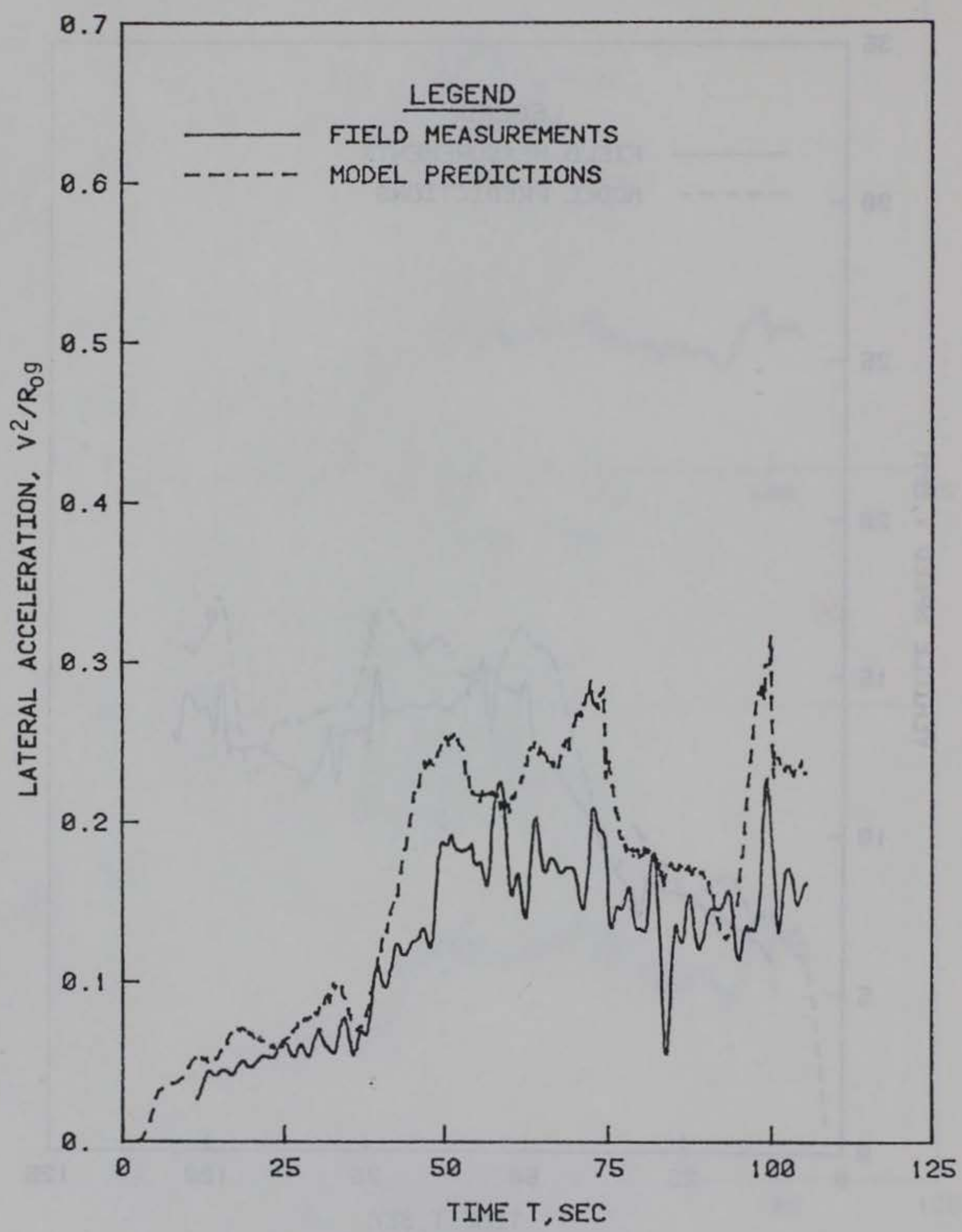

Figure 34. Lateral acceleration-time history for test 101; comparison of model predictions with experimental data 


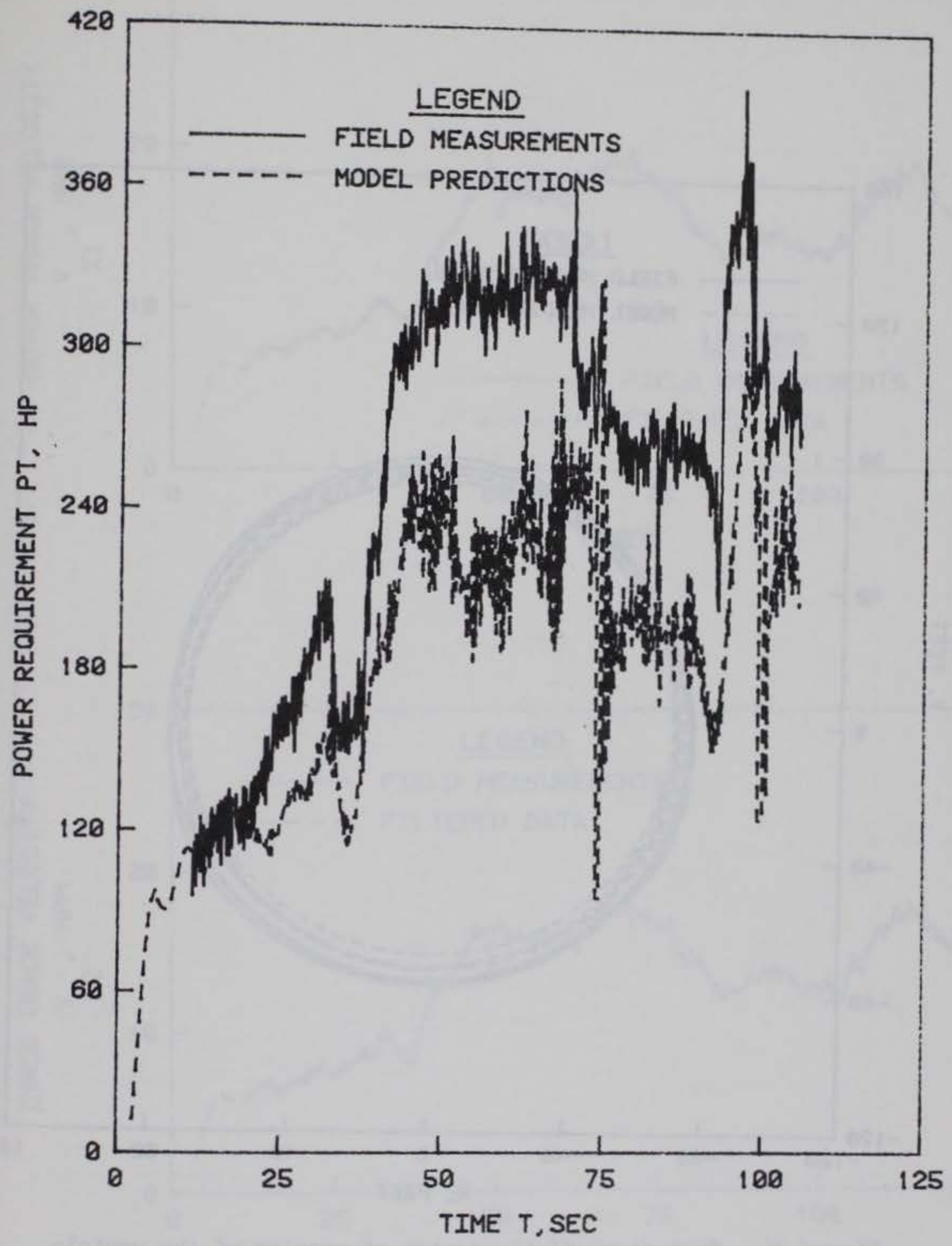

Figure 35. Total power-time history for test 101; comparison of model predictions with experimental data 


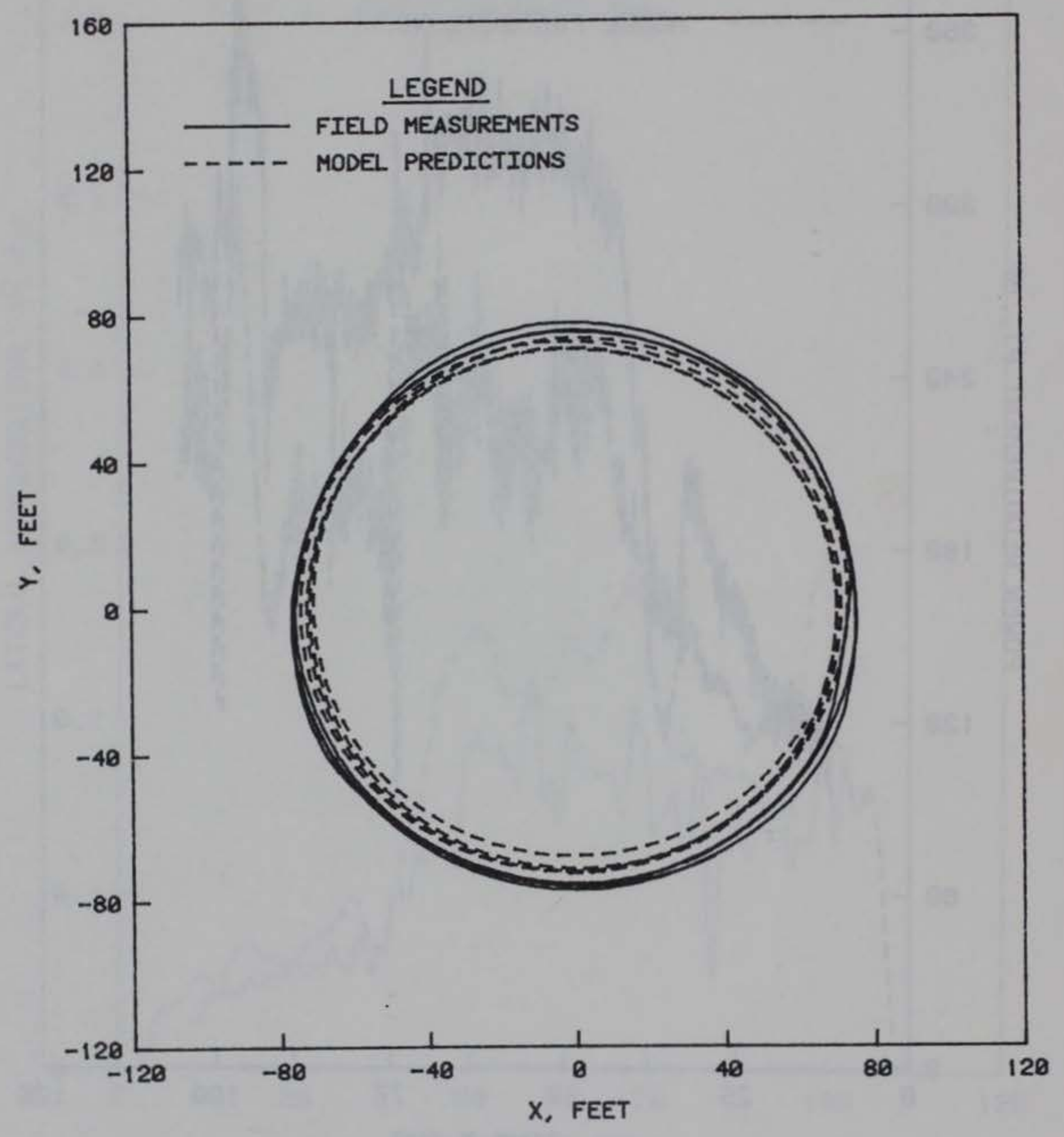

Figure 36. Trajectory of the center of gravity of the vehicle for test 101; comparison of model predictions with experimental data 

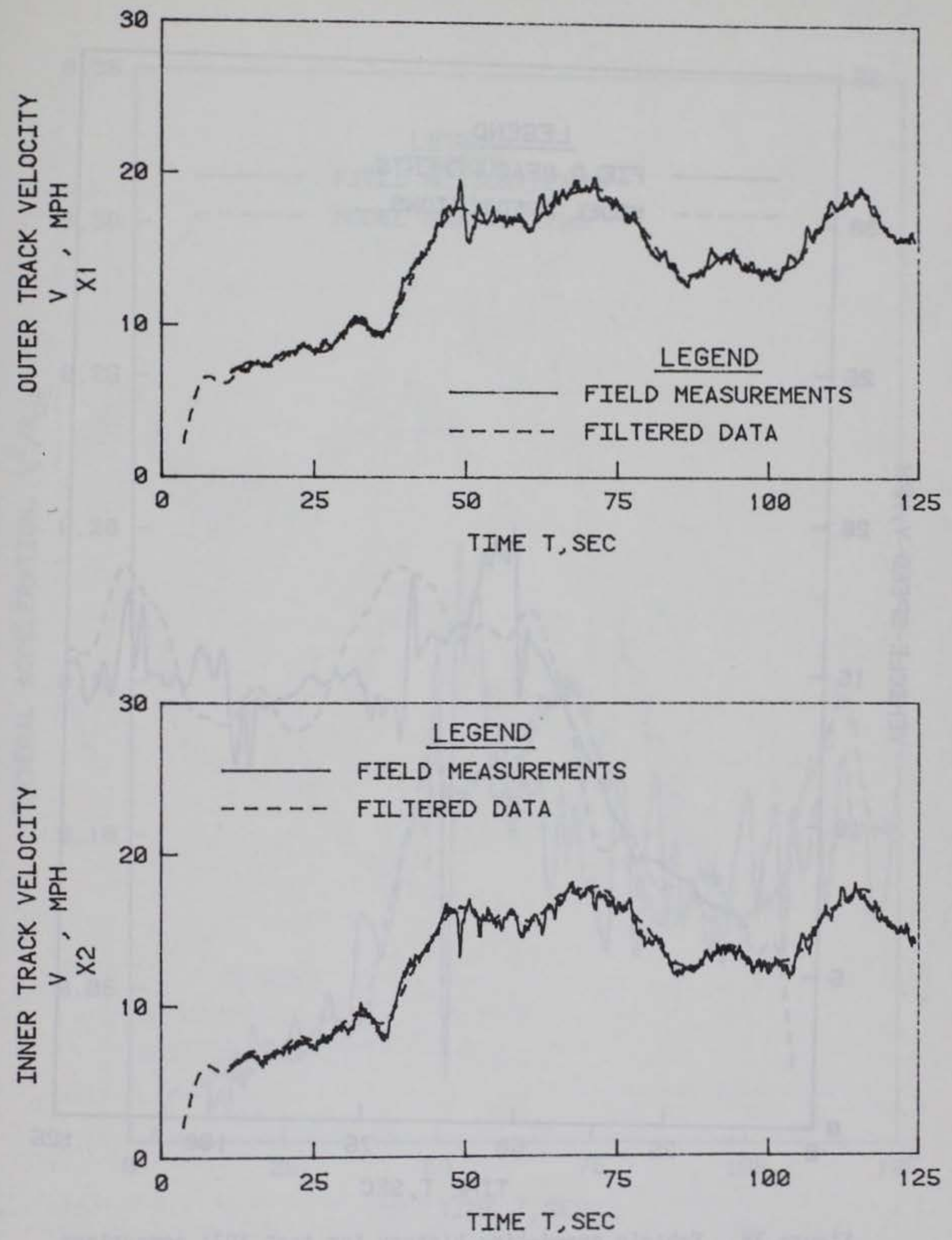

Figure 37. Outer and inner track velocity-time histories for test 102; field measurement and filtered data (input) 


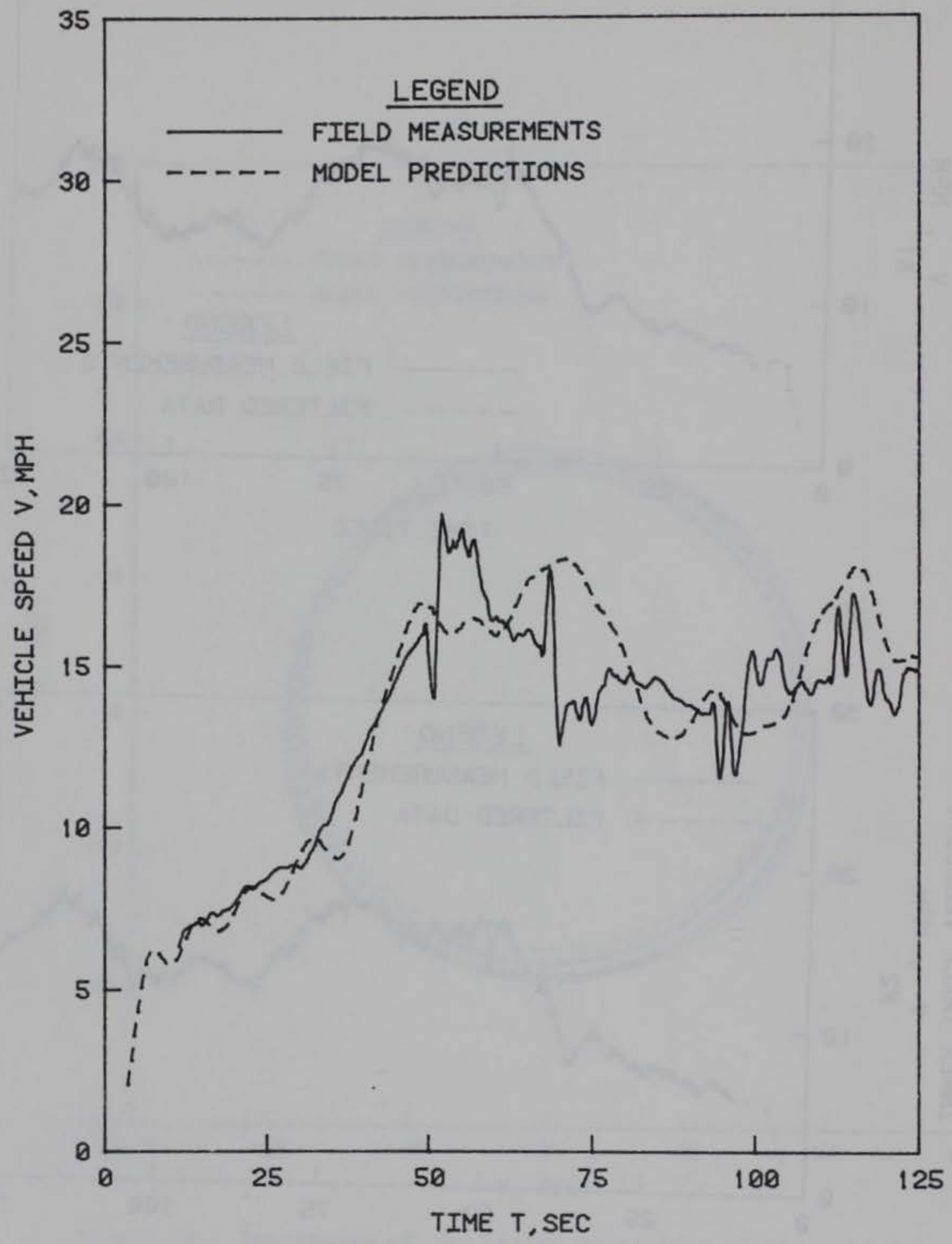

Figure 38. Vehicle speed-time history for test 102; comparison of model predictions with experimental data 


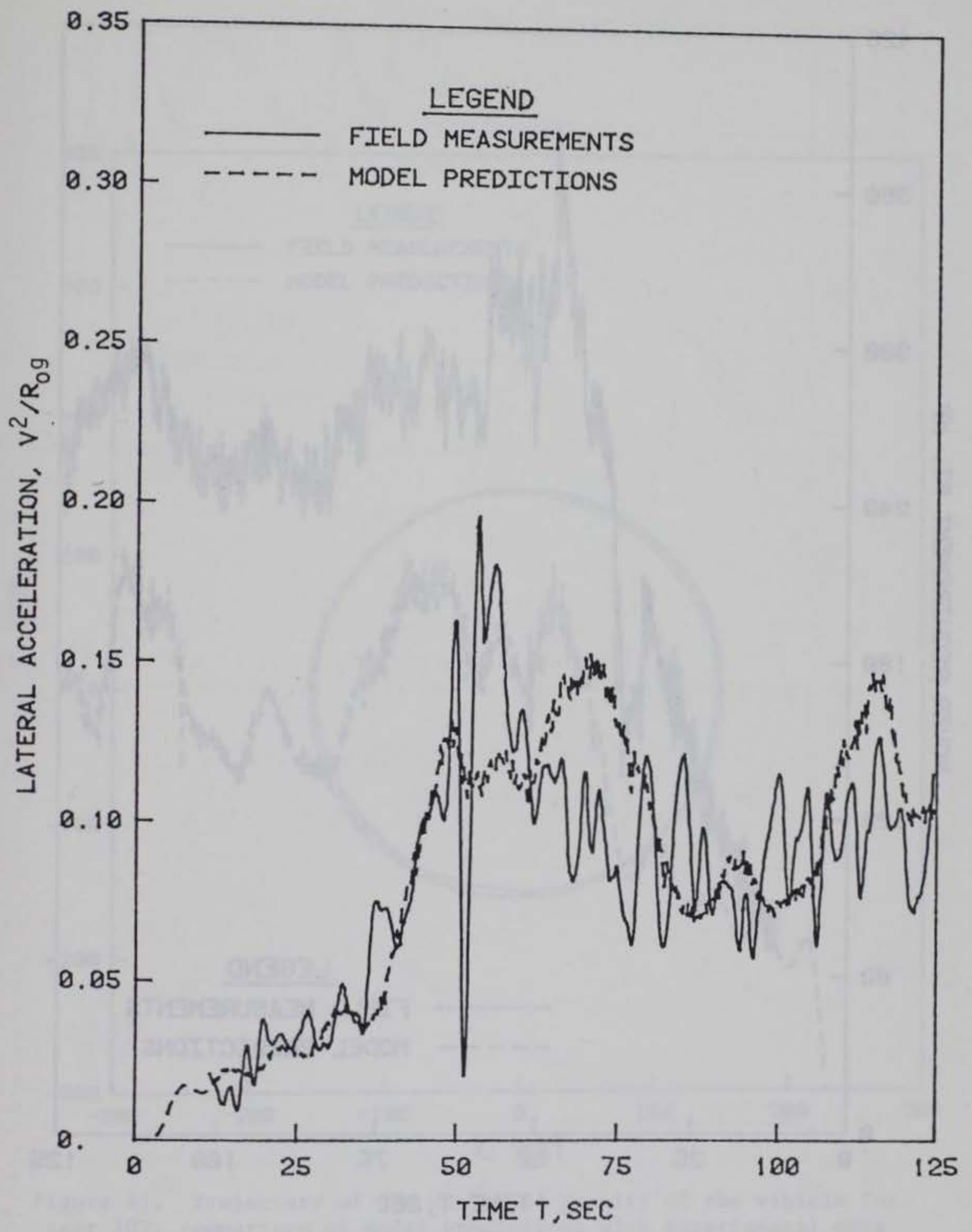

Figure 39. Lateral acceleration-time history for test 102; comparison of model predictions with experimental data 


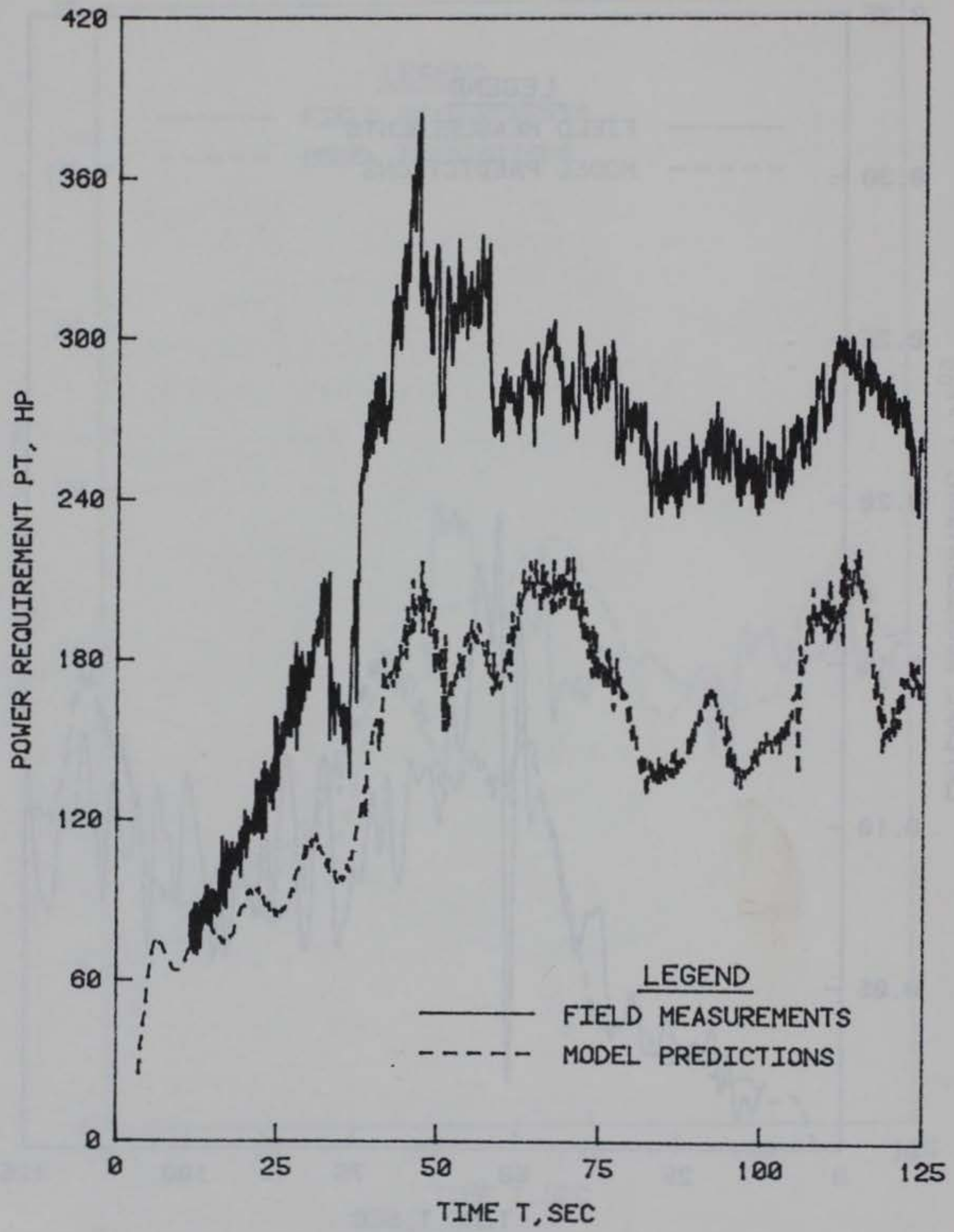

Figure 40. Total power-time history for test 102; comparison of model predictions with experimental data 


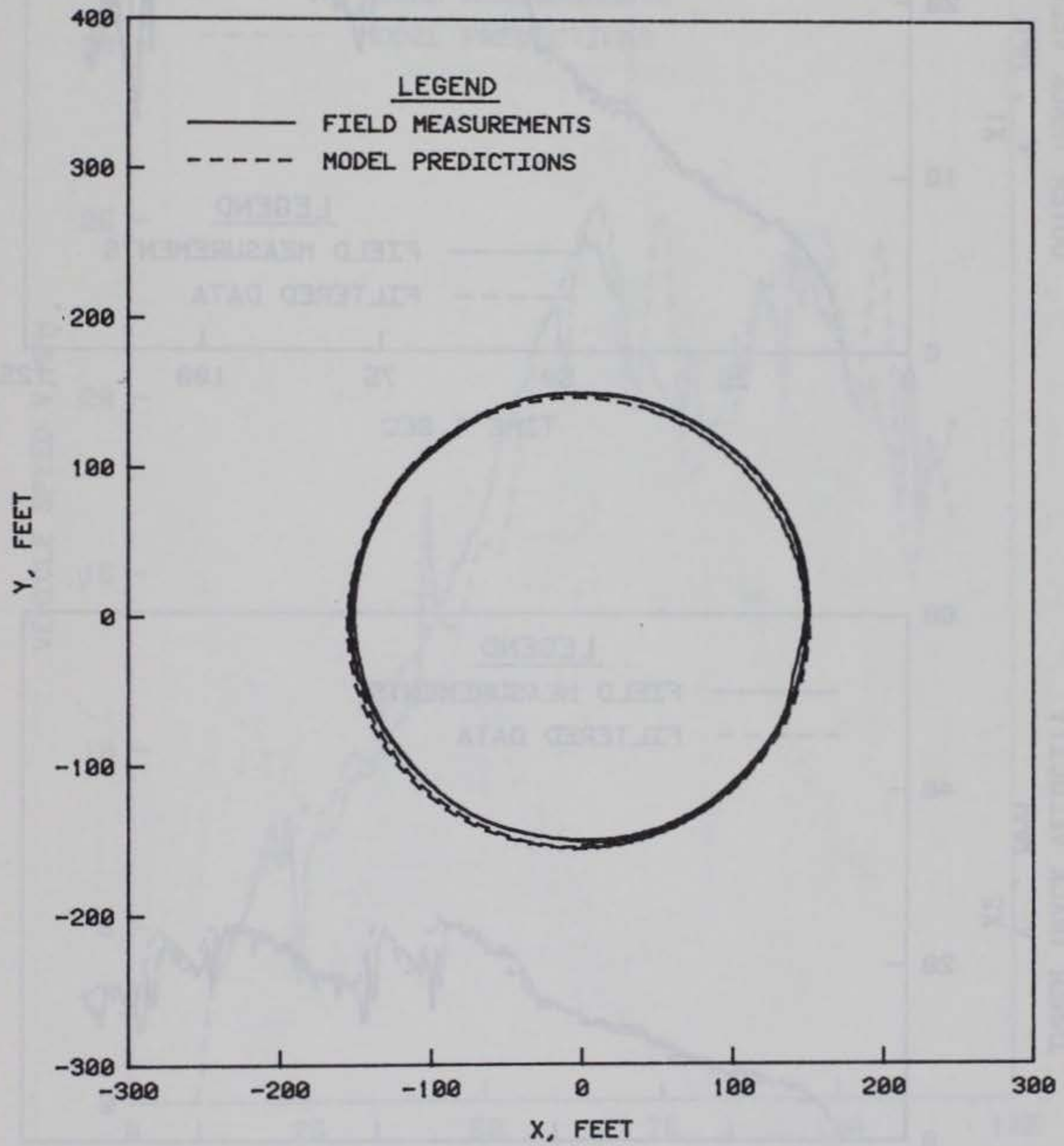

Figure 41. Trajectory of the center of gravity of the vehicle for test 102; comparison of model predictions with experimental data 

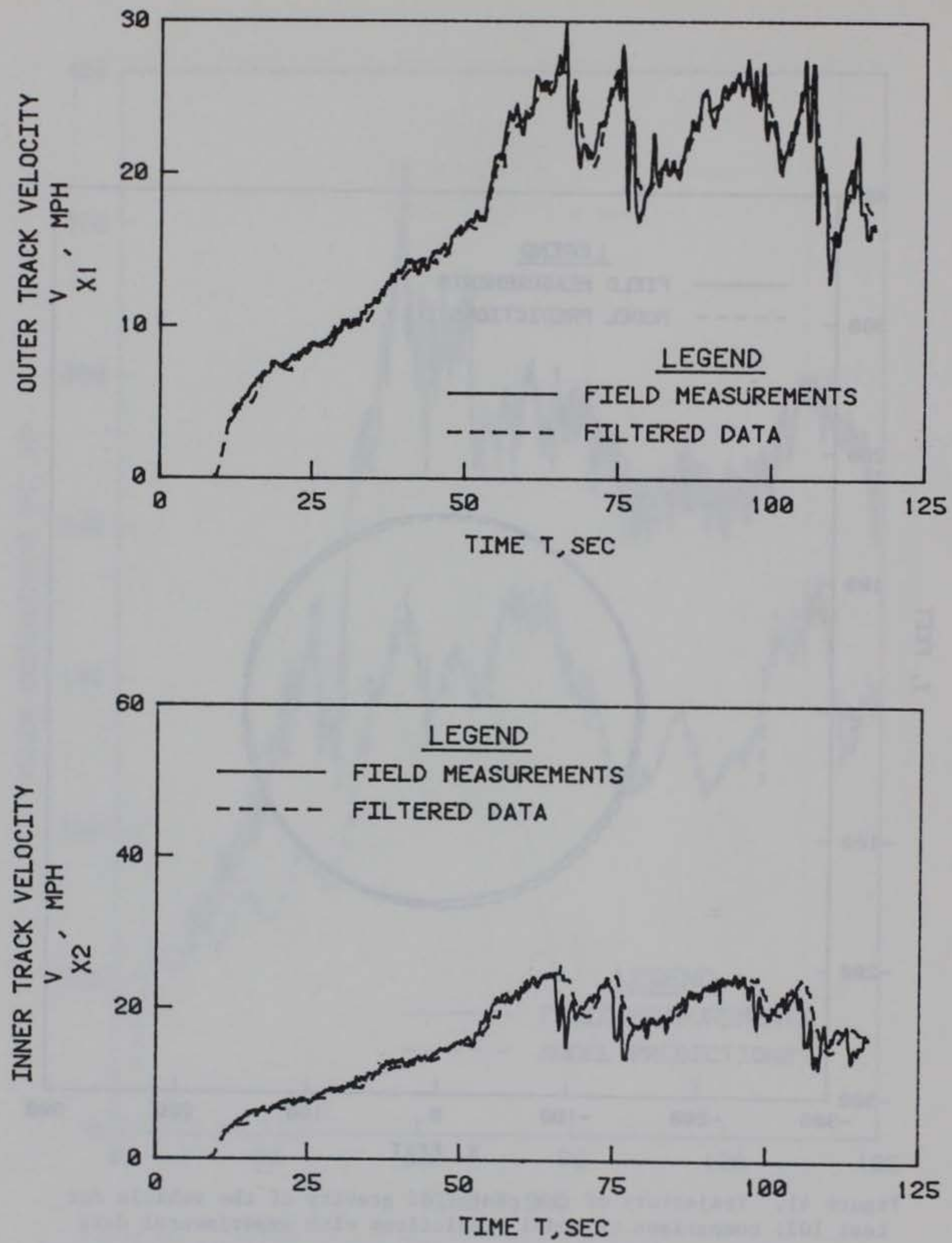

Figure 42. Outer and inner track velocity-time histories for test 107; field measurement and filtered data (input) 


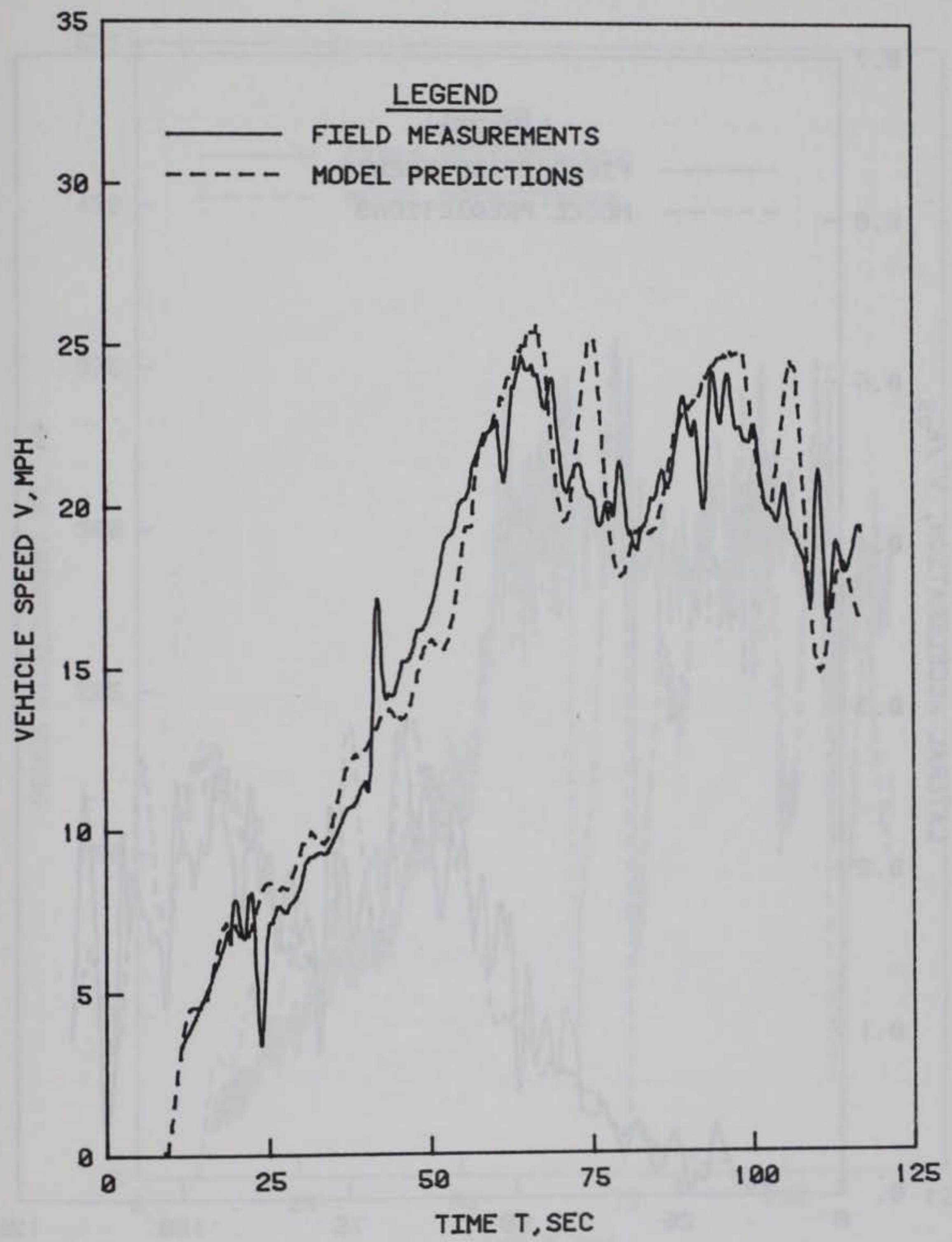

Figure 43. Vehicle speed-time history for test 107; comparison of model predictions with experimental data 


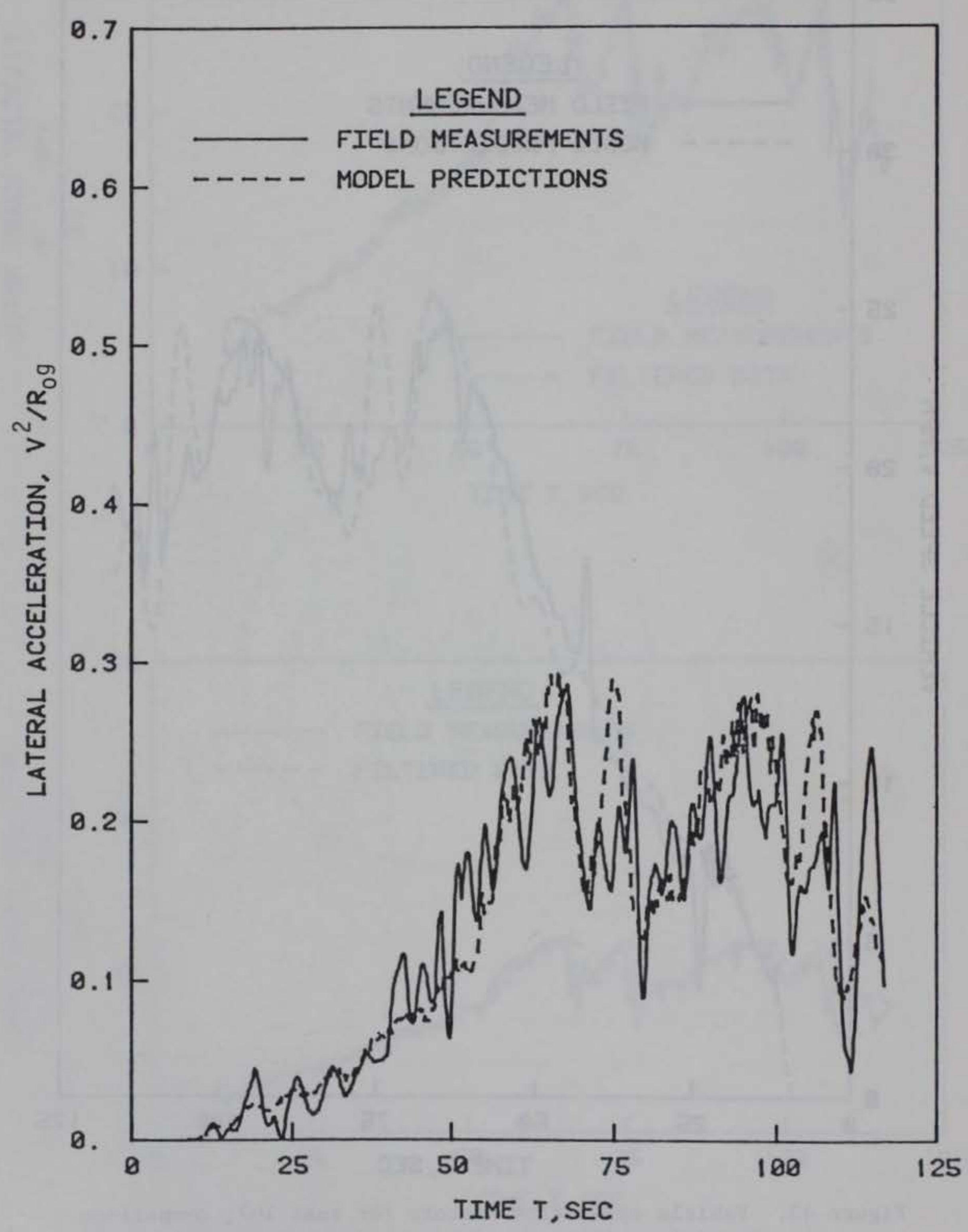

Figure 44. Lateral acceleration-time history for test 107; comparison of model predictions with experimental data 


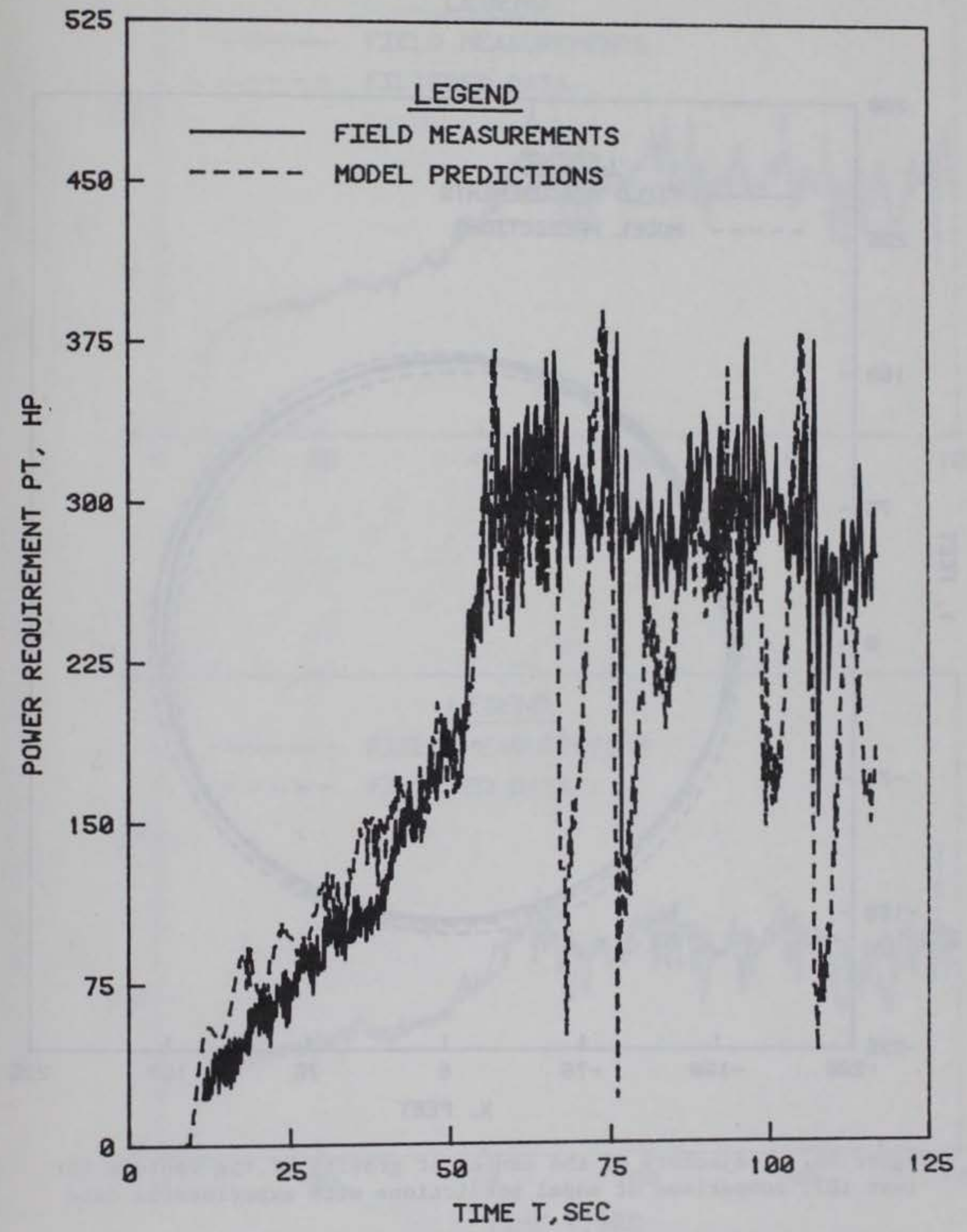

Figure 45. Total power-time history for test 107; comparison of model predictions with experimental data 


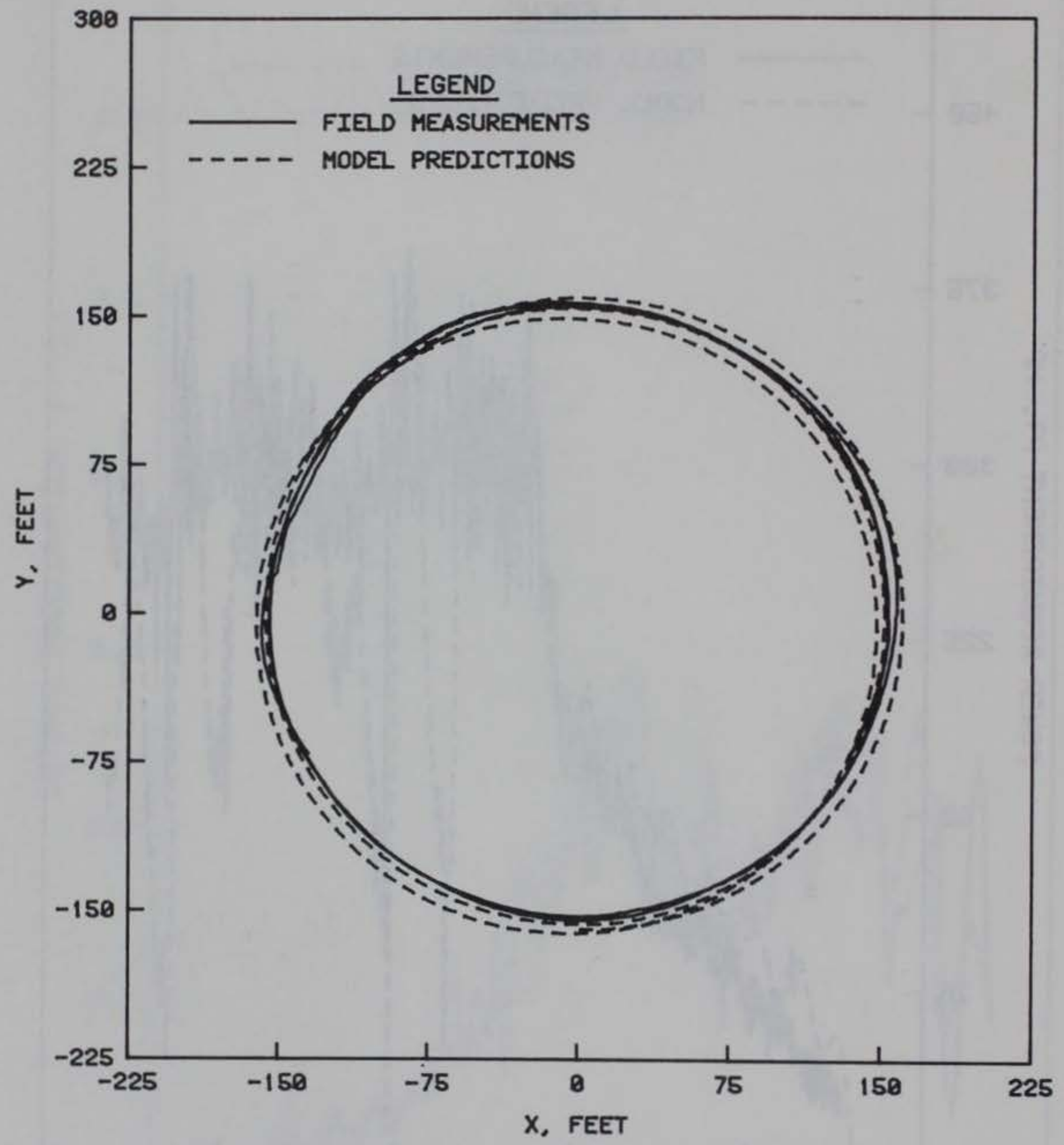

Figure 46. Trajectory of the center of gravity of the vehicle for test 107; comparison of model predictions with experimental data 

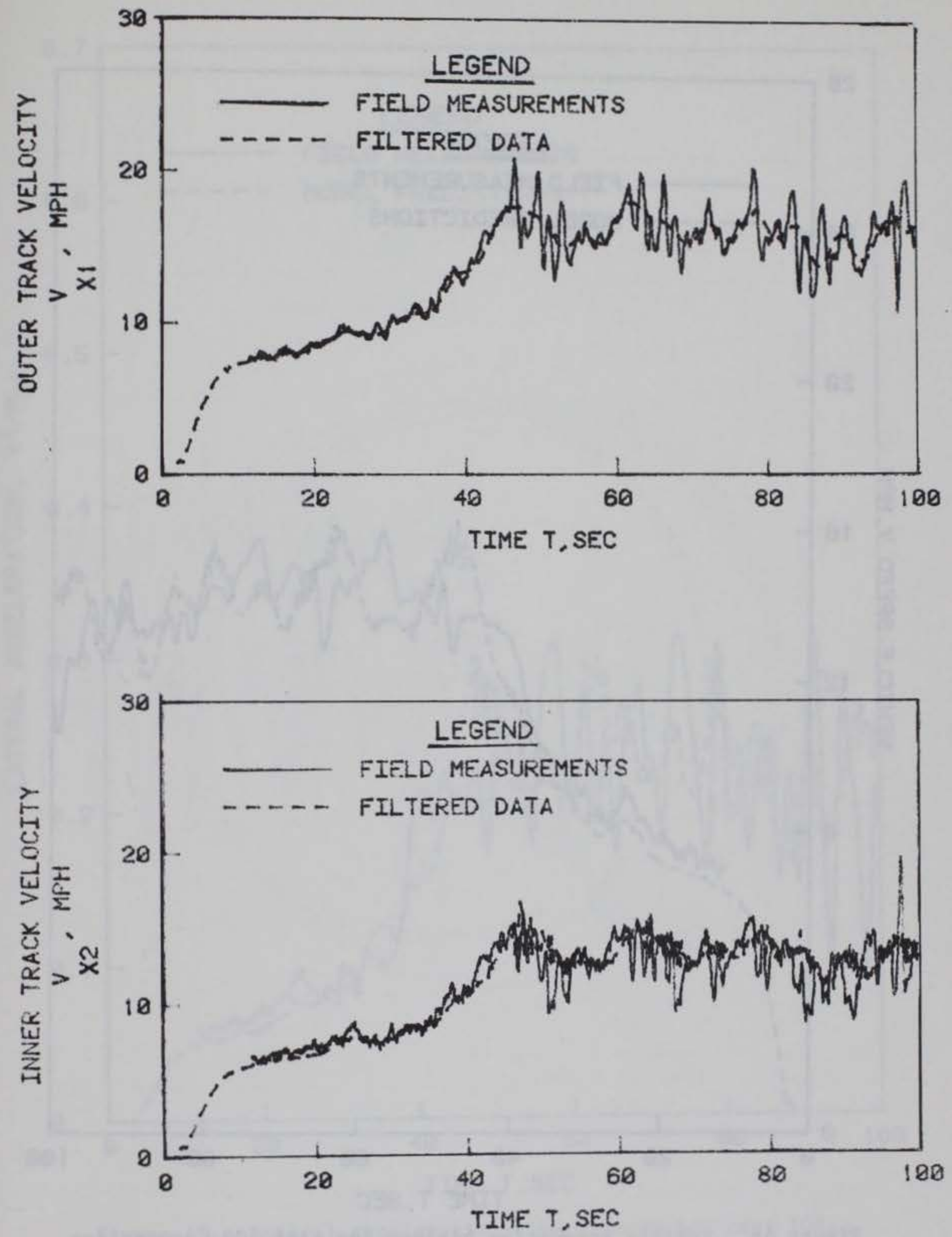

Figure 47. Outer and inner track velocity-time histories for test 108; field measurement and filtered data (input) 


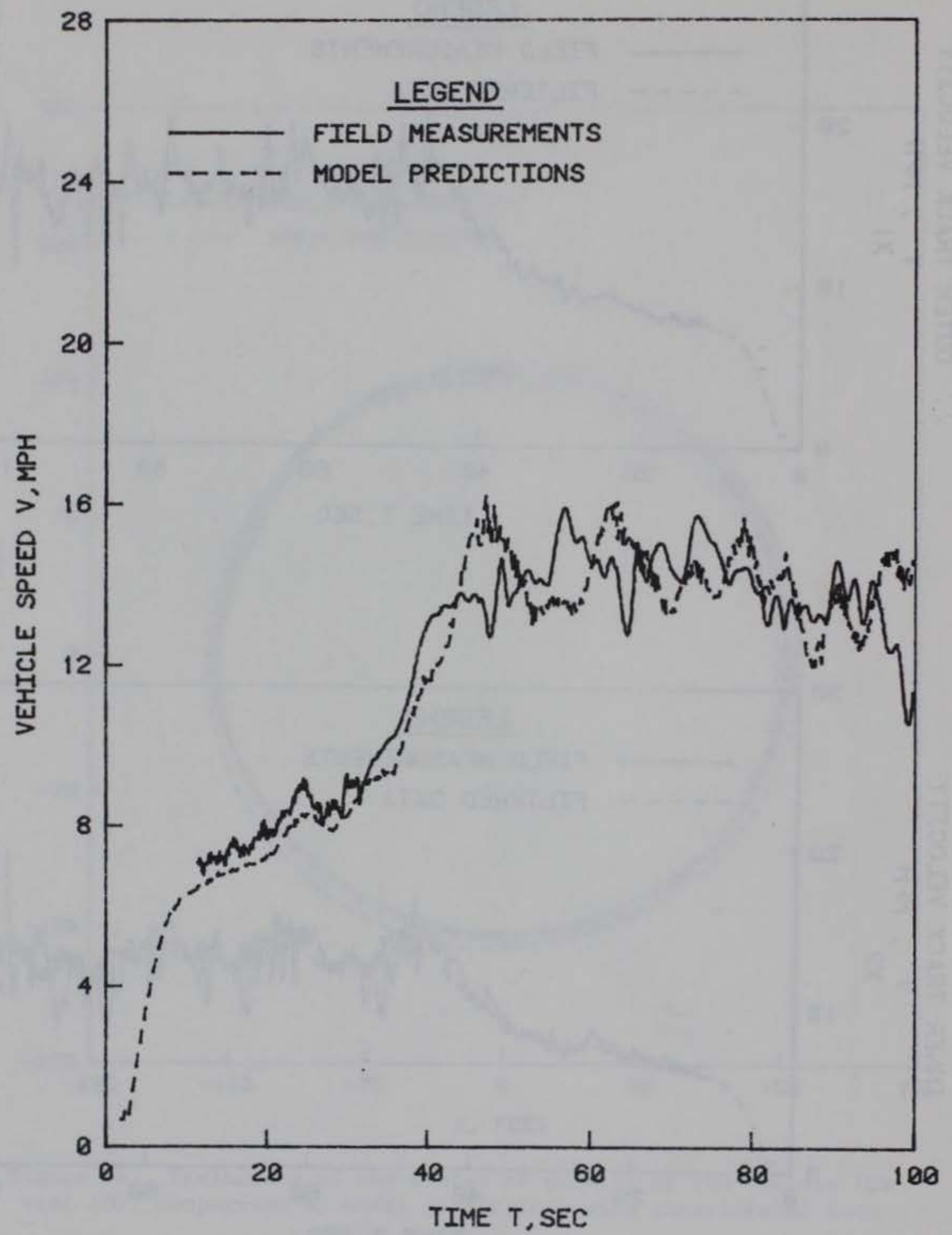

Figure 48. Vehicle speed-time history for test 108; comparison of model predictions with experimental data 


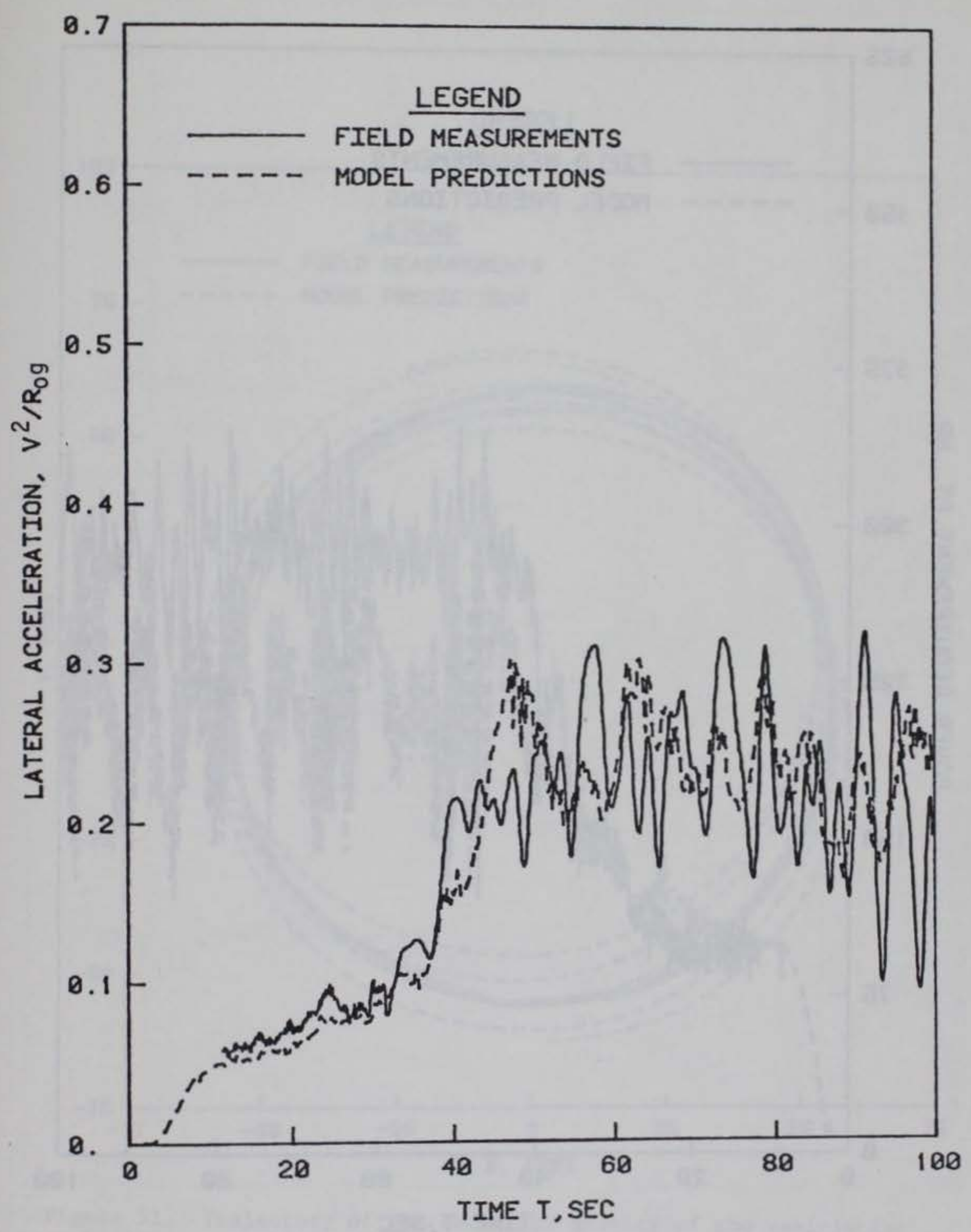

Figure 49. Lateral acceleration-time history for test 108; comparison of model predictions with experimental data 


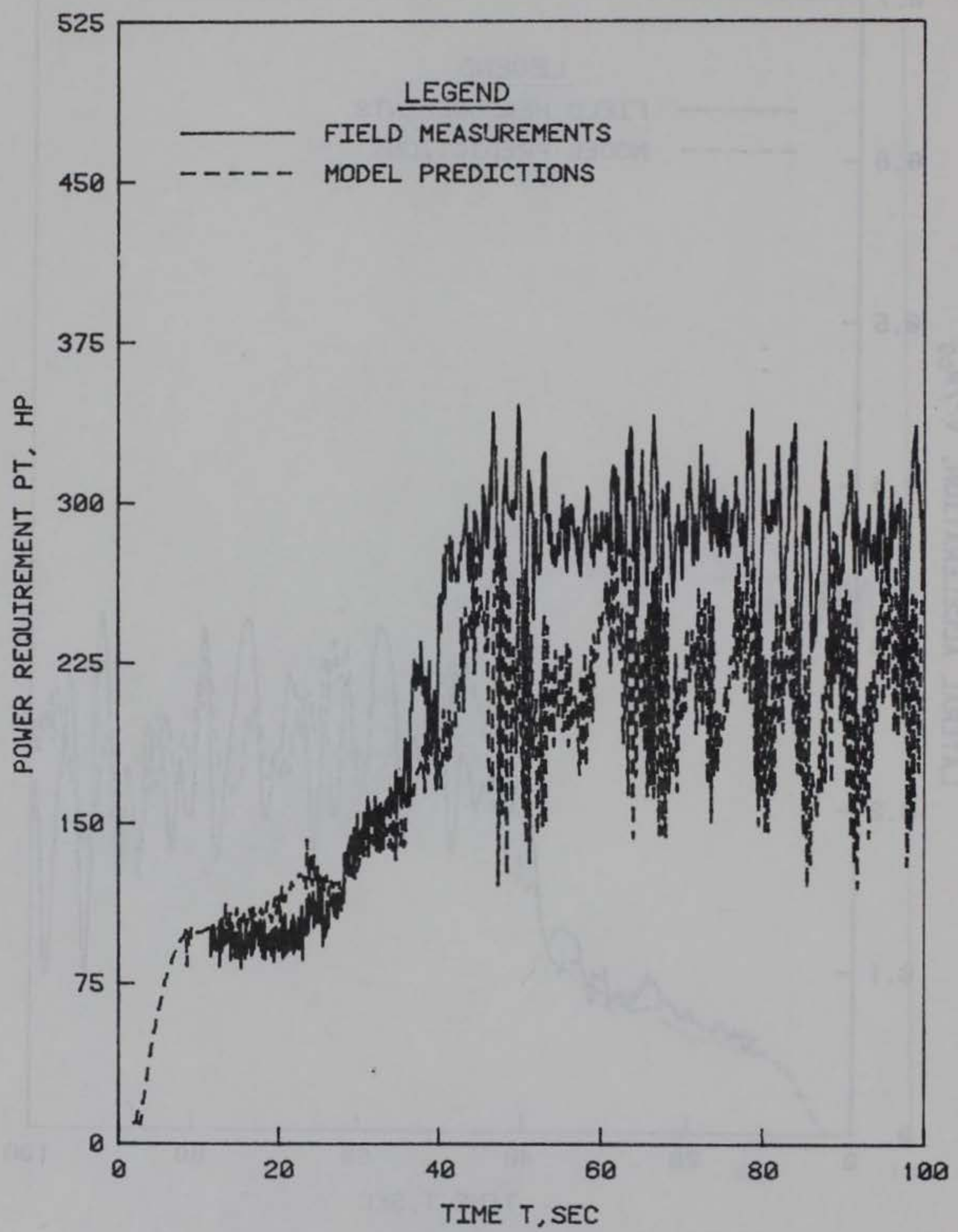

Figure 50. Total power-time history for test 108; comparison of model predictions with experimental data 


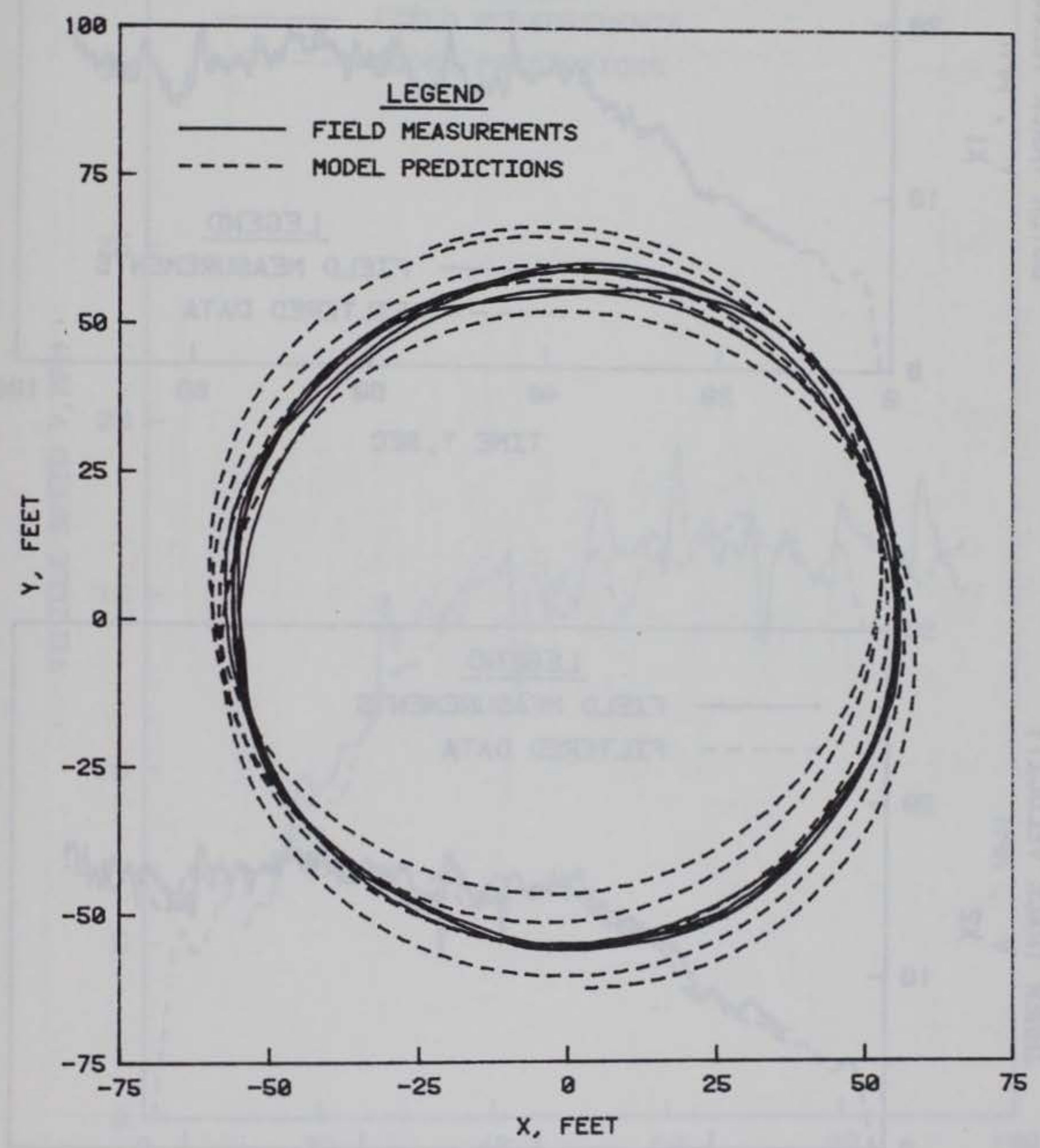

Figure 51. Trajectory of the center of gravity of the vehicle for test 108; comparison of model predictions with experimental data 

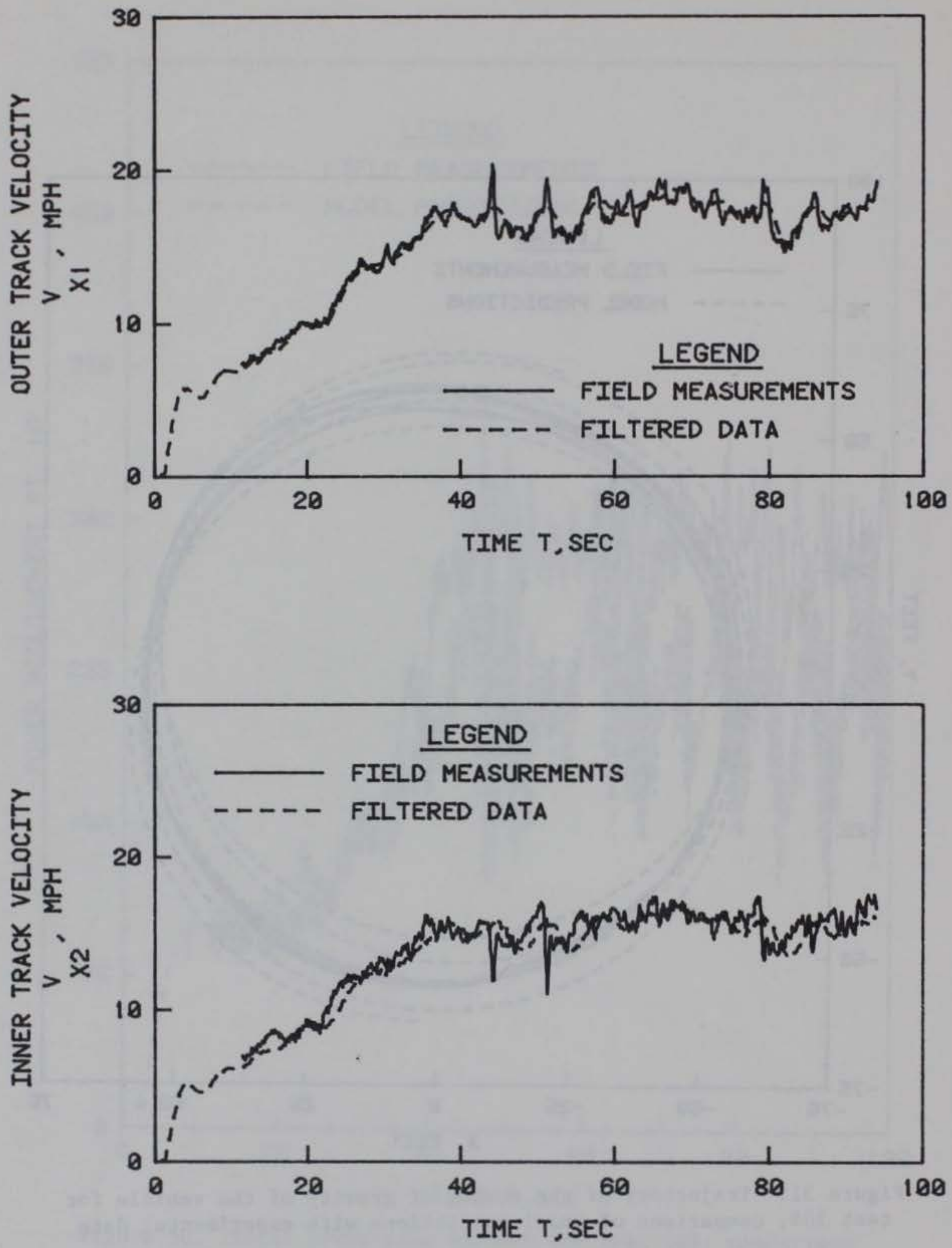

Figure 52. Outer and inner track velocity-time histories for test 109; field measurement and filtered data (input) 


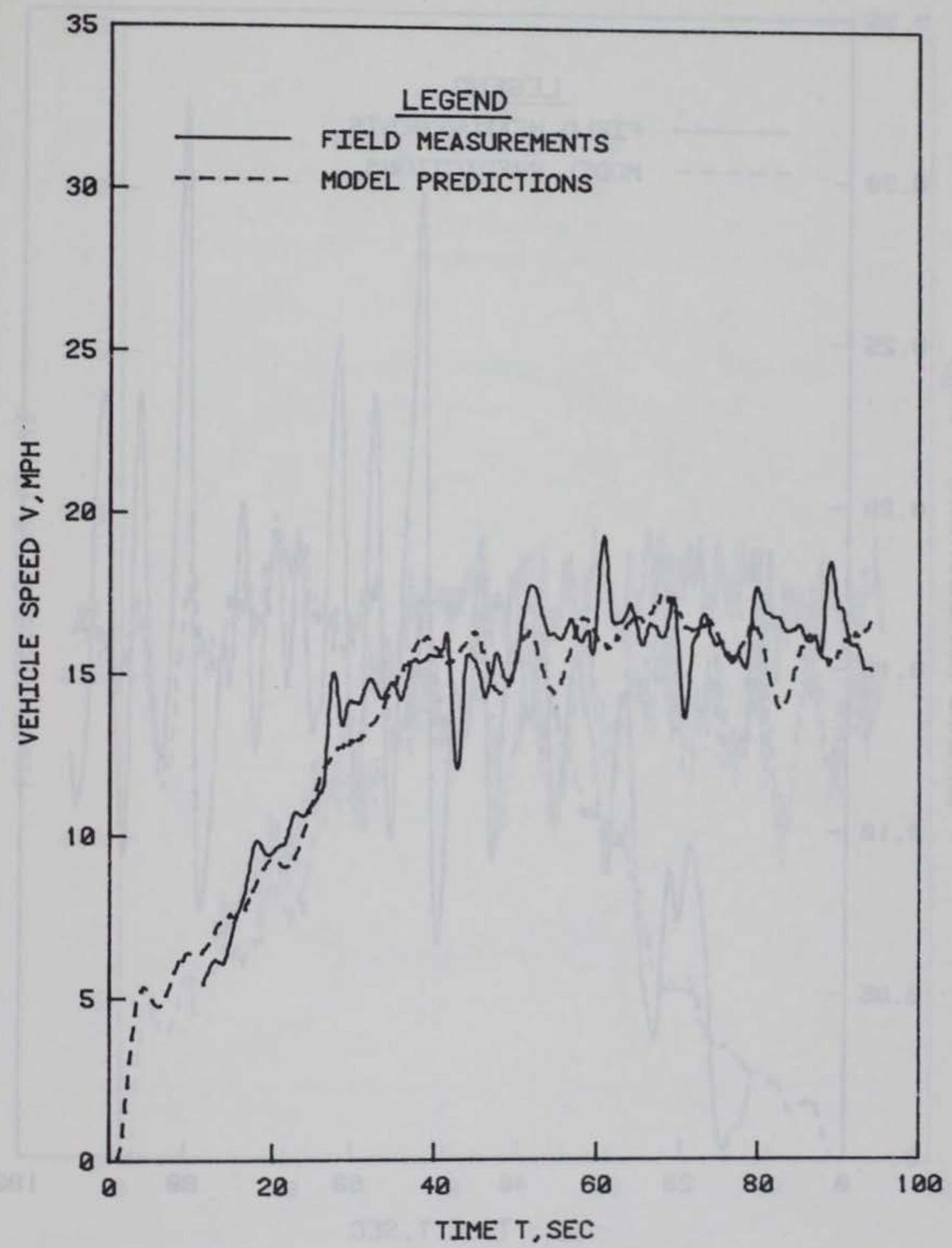

Figure 53. Vehicle speed-time history for test 109; comparison of model predictions with experimental data 


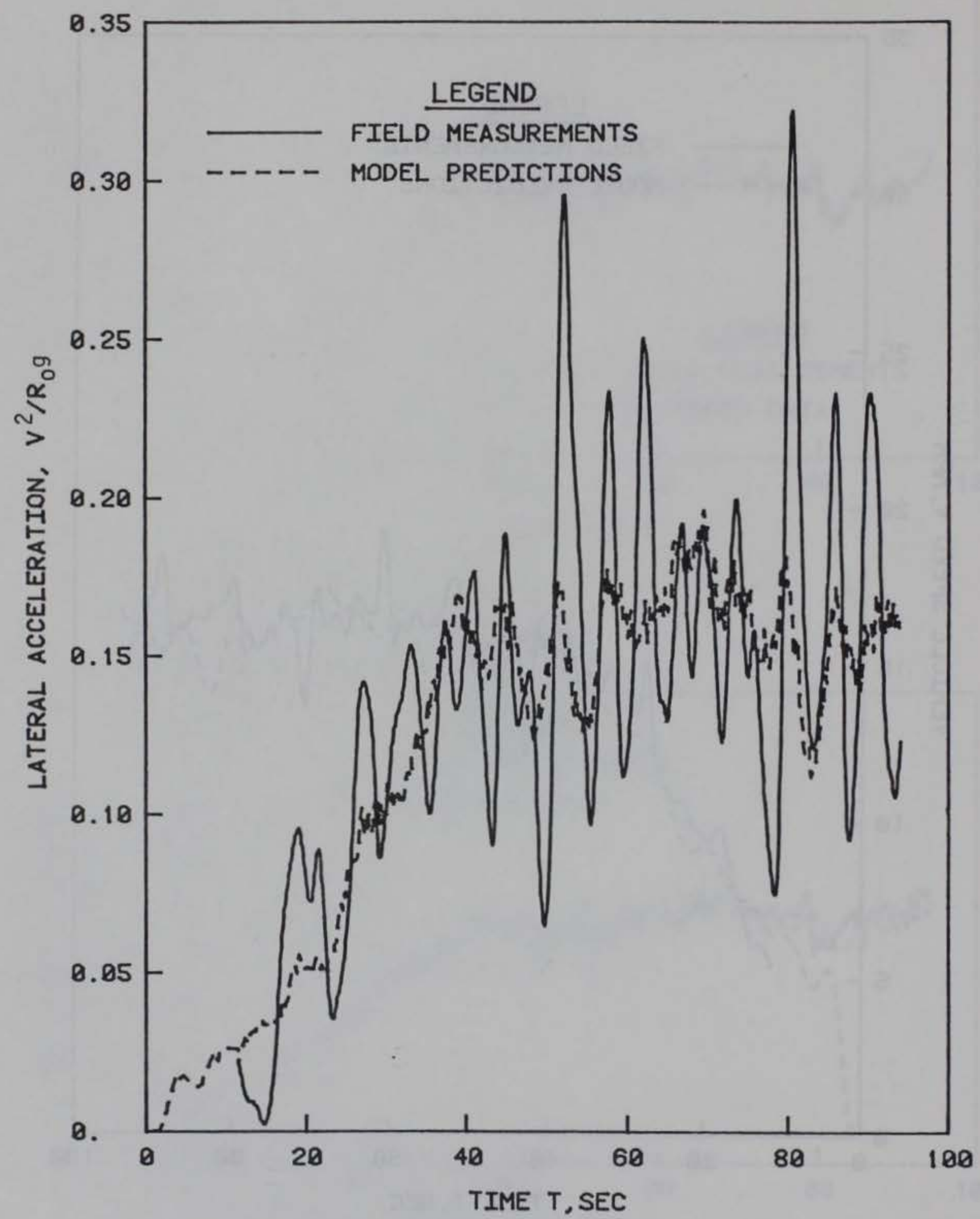

Figure 54. Lateral acceleration-time history for test 109; comparison of model predictions with experimental data 


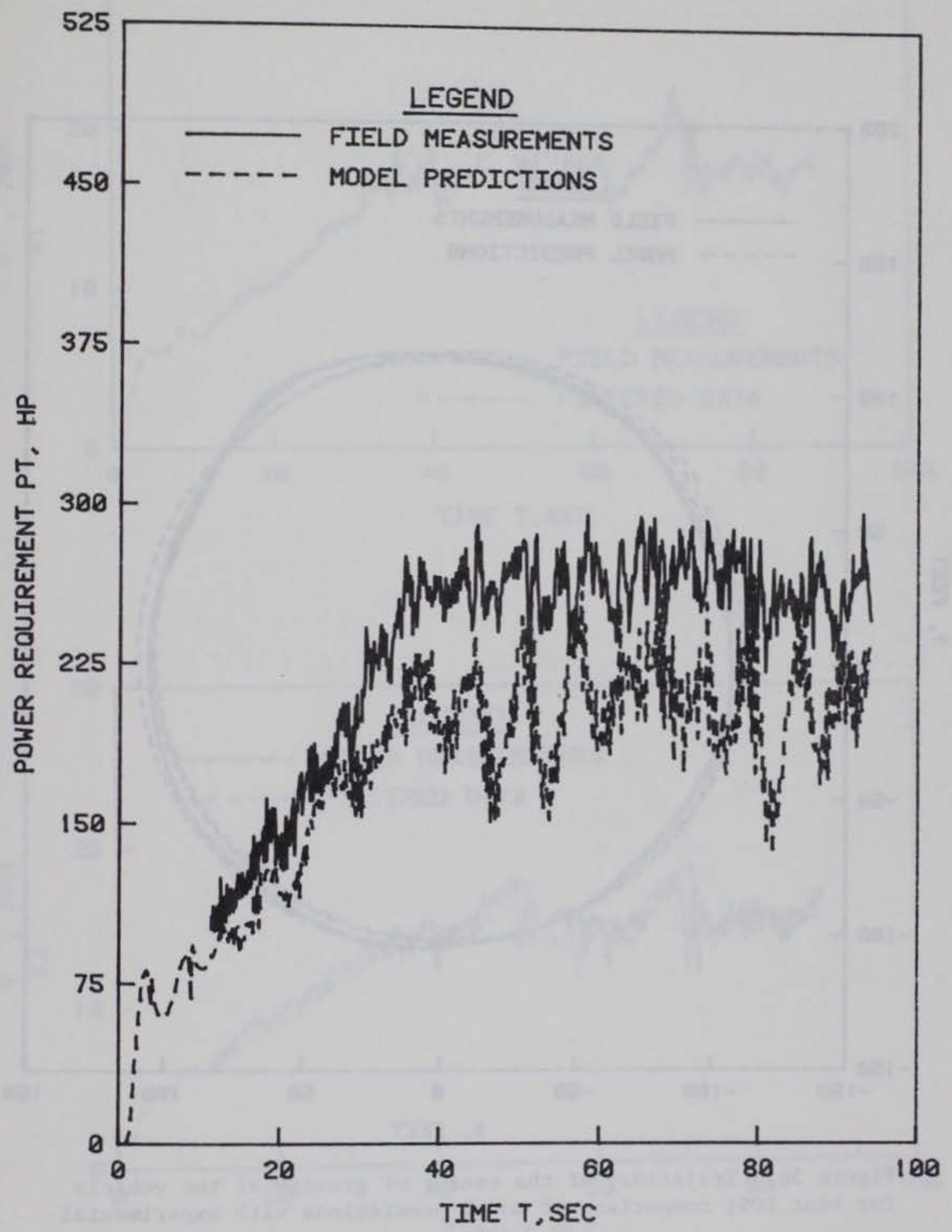

Figure 55. Total power-time history for test 109; comparison of model predictions with experimental data 


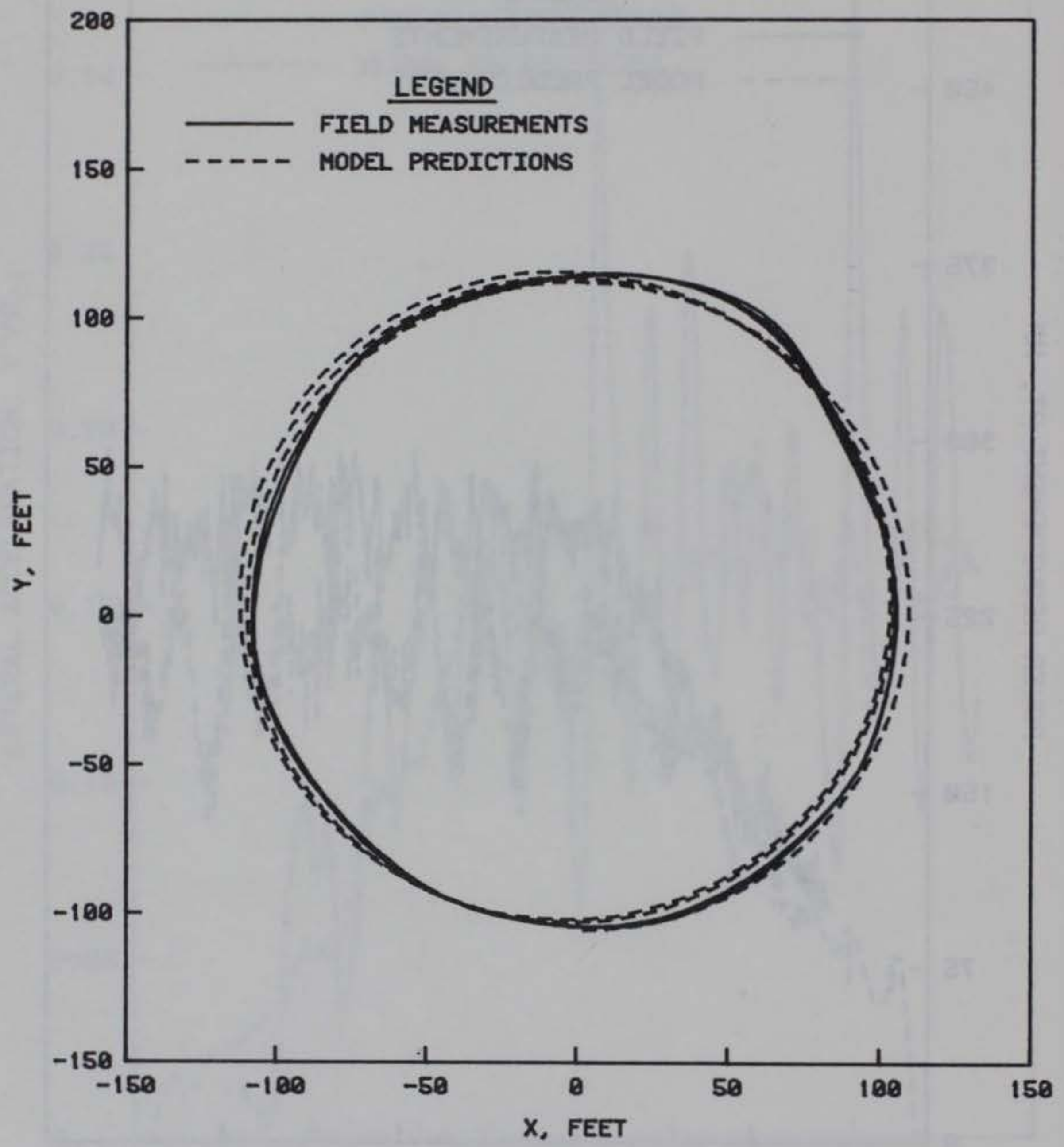

Figure 56. Trajectory of the center of gravity of the vehicle for test 109; comparison of model predictions with experimental data 

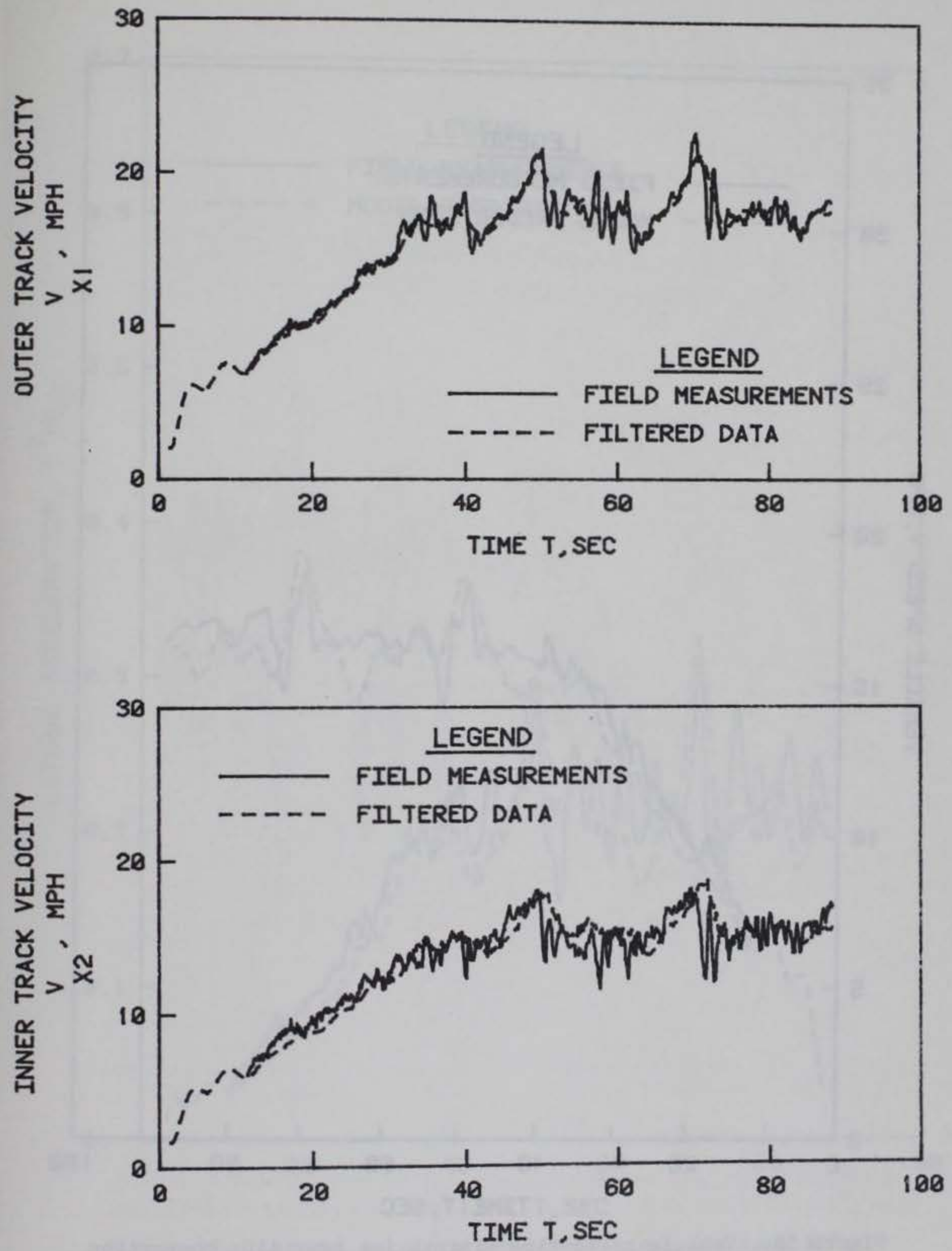

Figure 57. Outer and inner track velocity-time histories for test 110; field measurement and filtered data (input) 


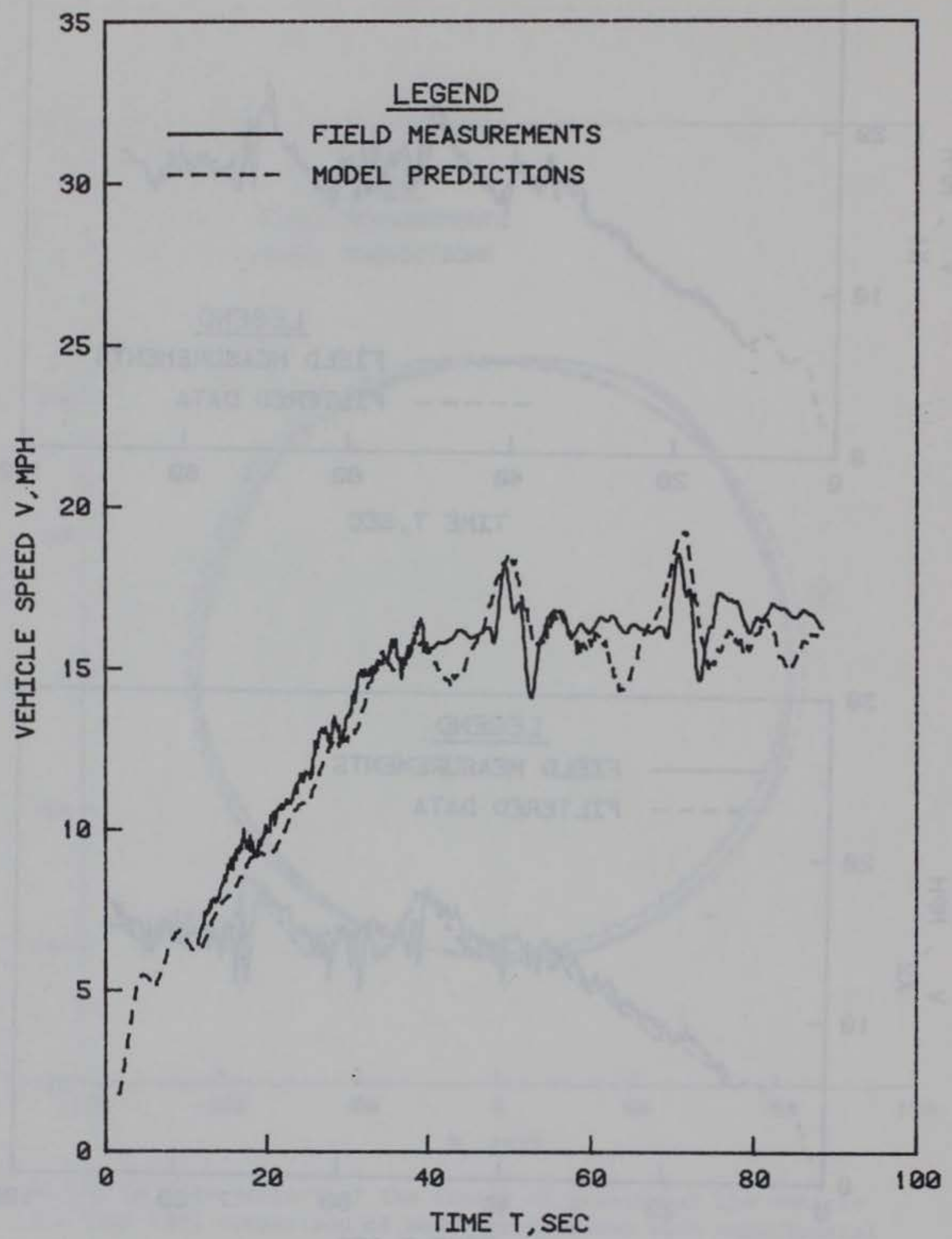

Figure 58. Vehicle speed-time history for test 110; comparison of model predictions with experimental data 


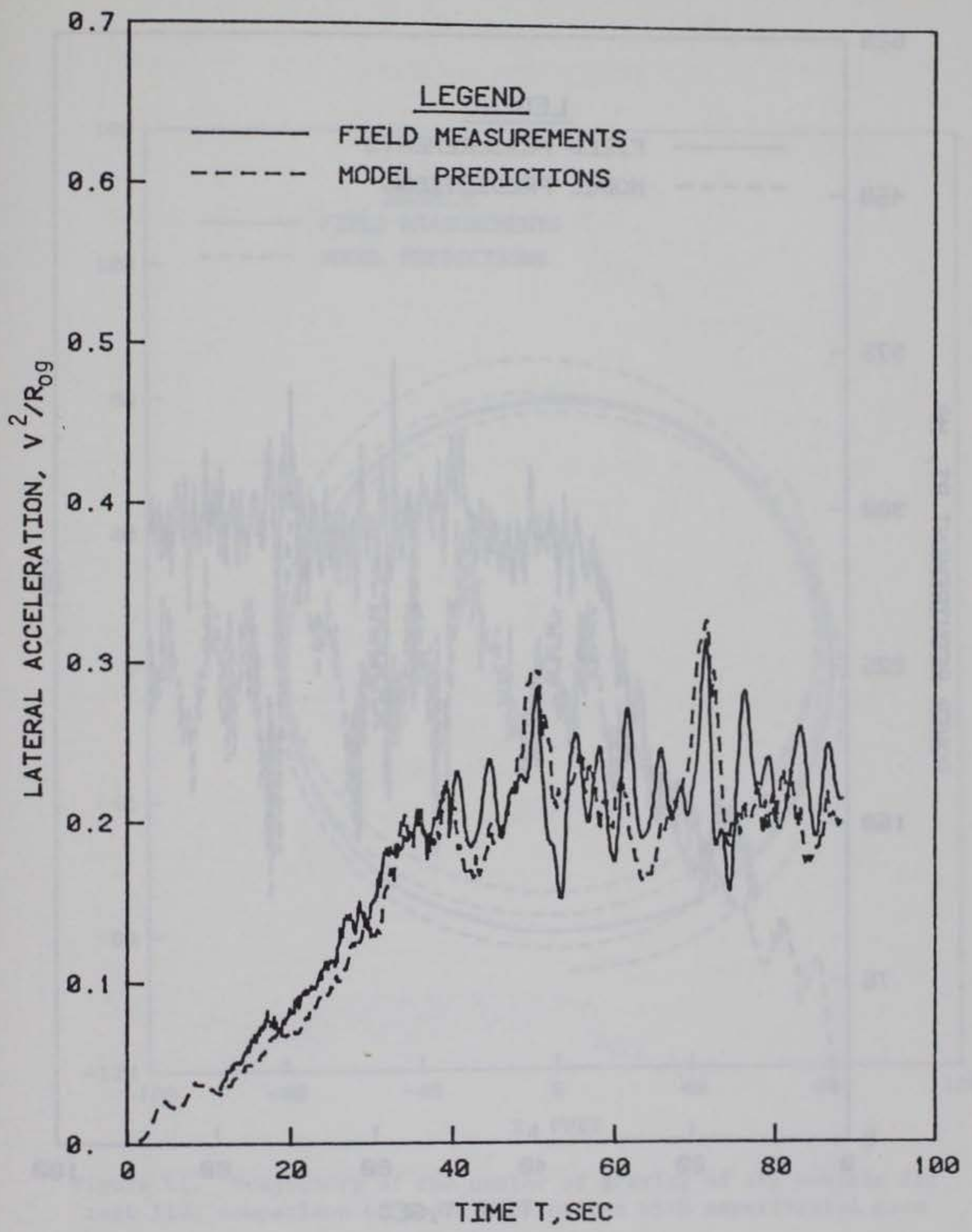

Figure 59. Lateral acceleration-time history for test 110; comparison of model predictions with experimental data 


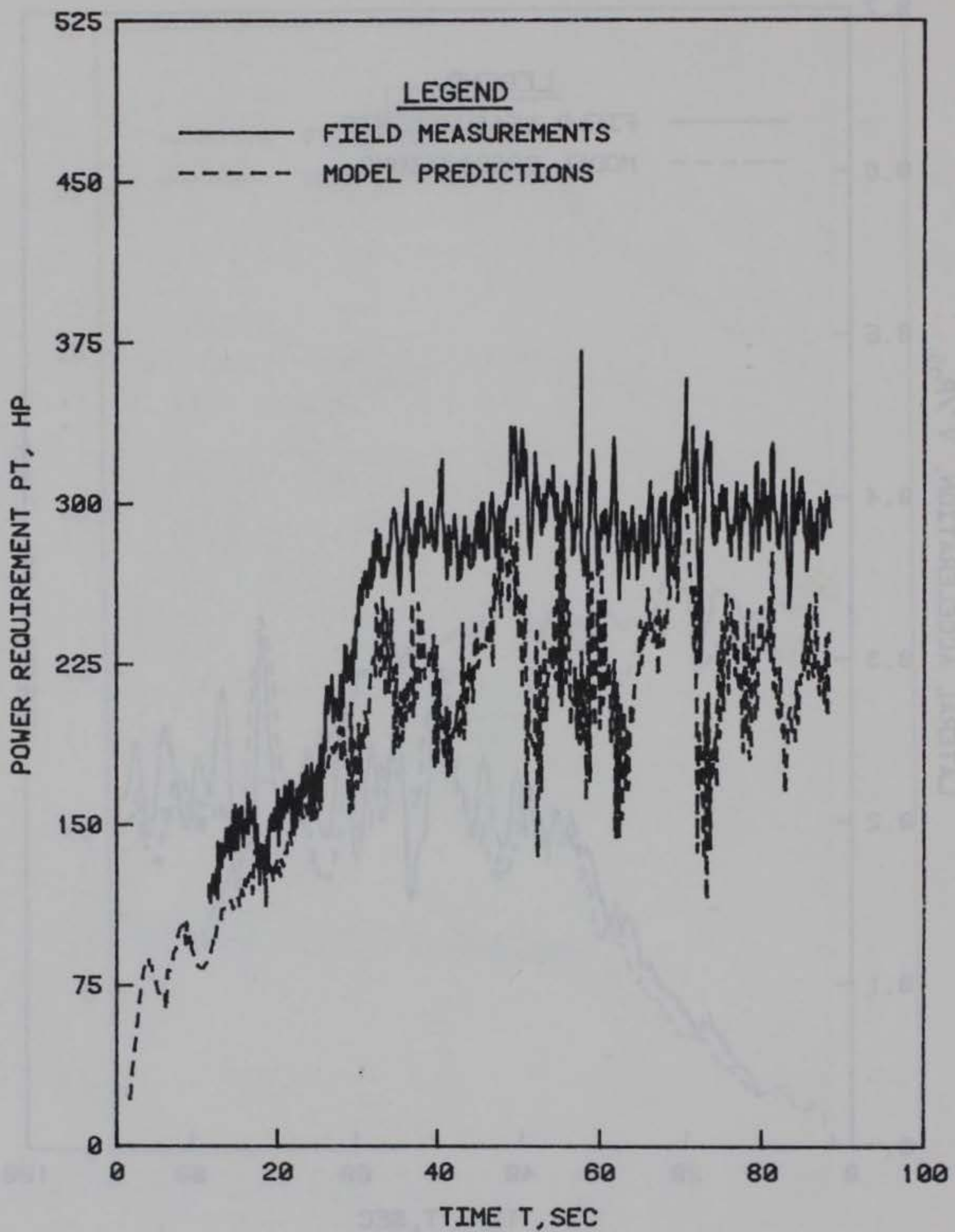

Figure 60. Total power-time history for test 110; comparison of model predictions with experimental data 


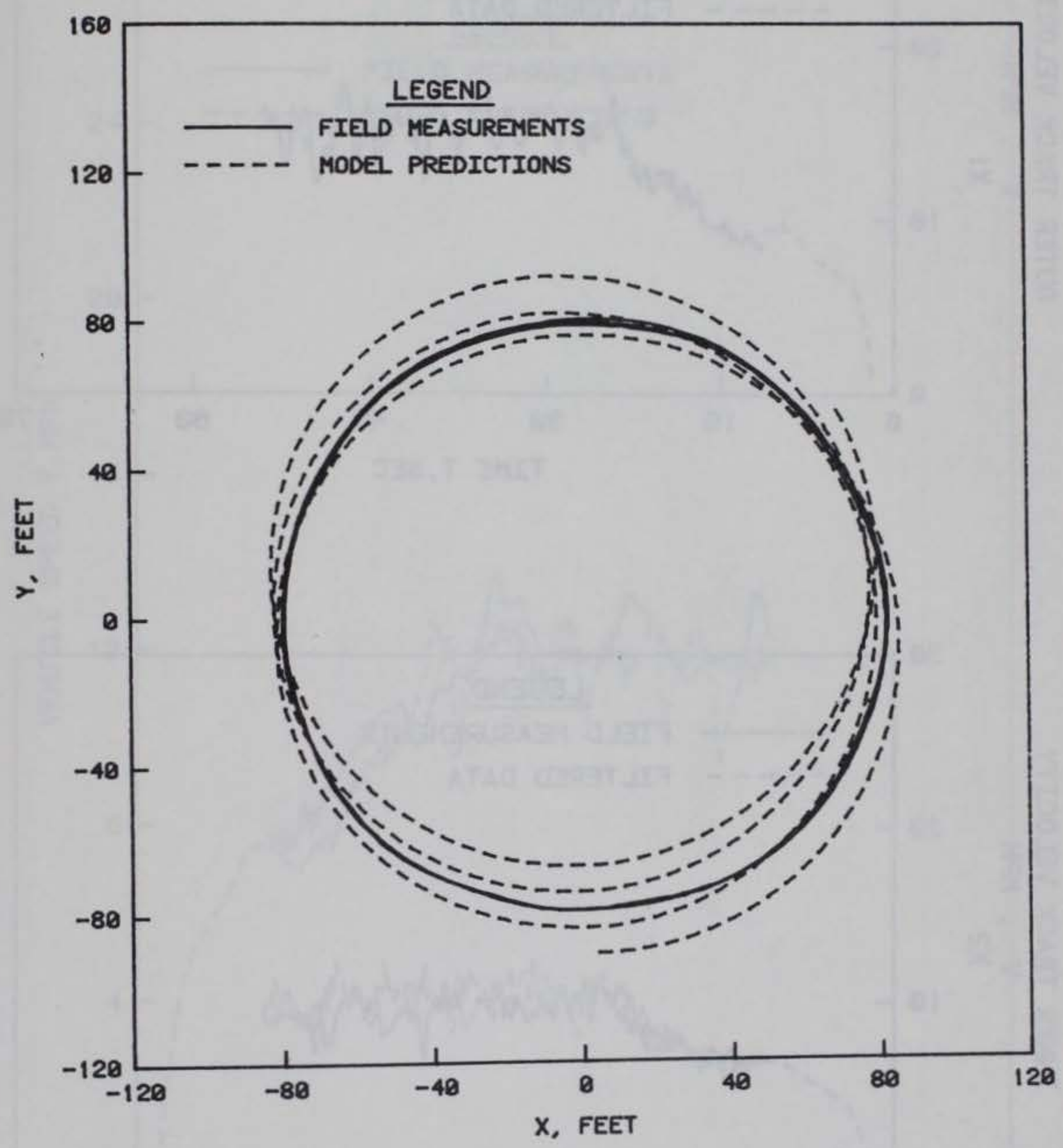

Figure 61. Trajectory of the center of gravity of the vehicle for test 110; comparison of model predictions with experimental data 

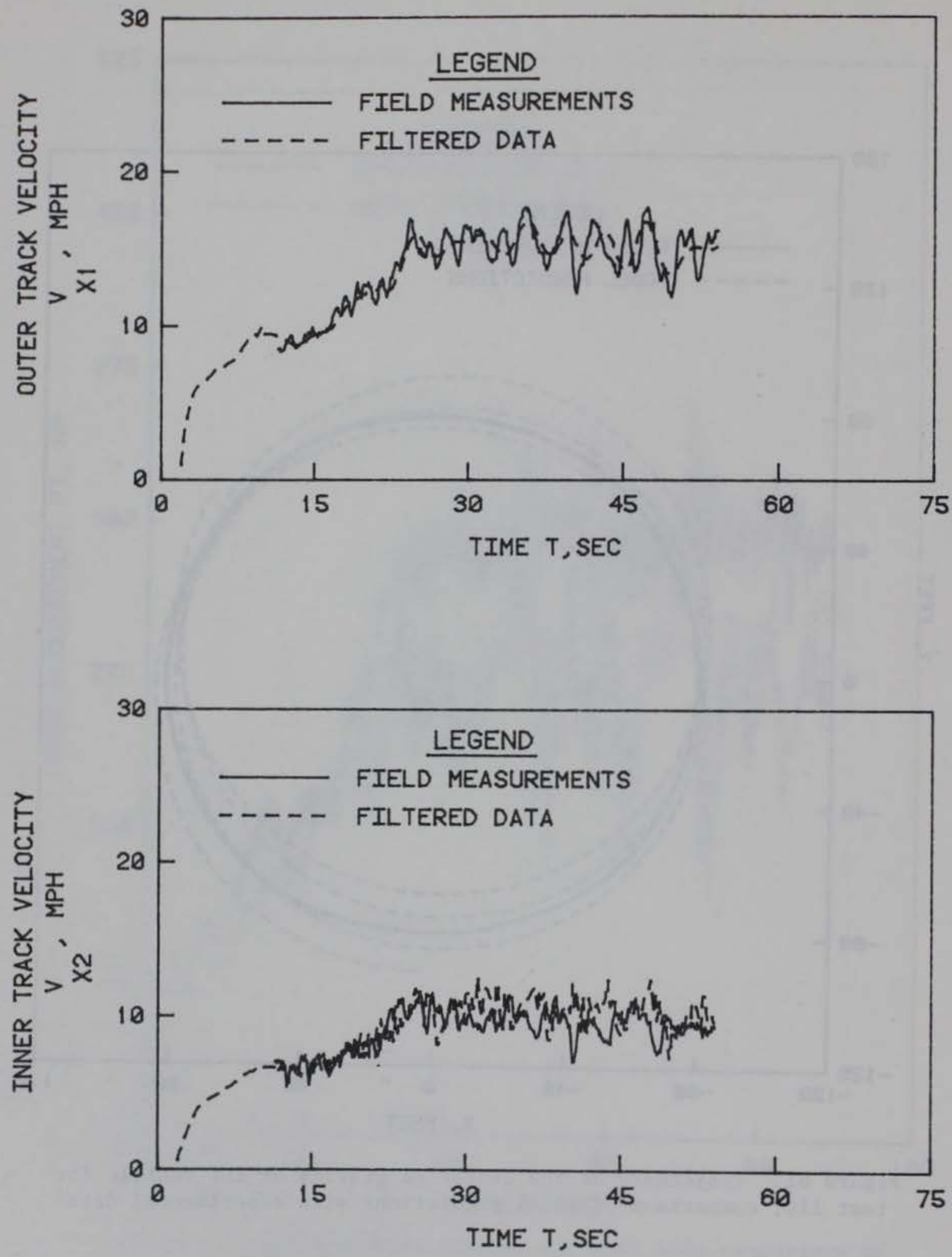

Figure 62. Outer and inner track velocity-time histories for test 111; field measurement and filtered data (input) 


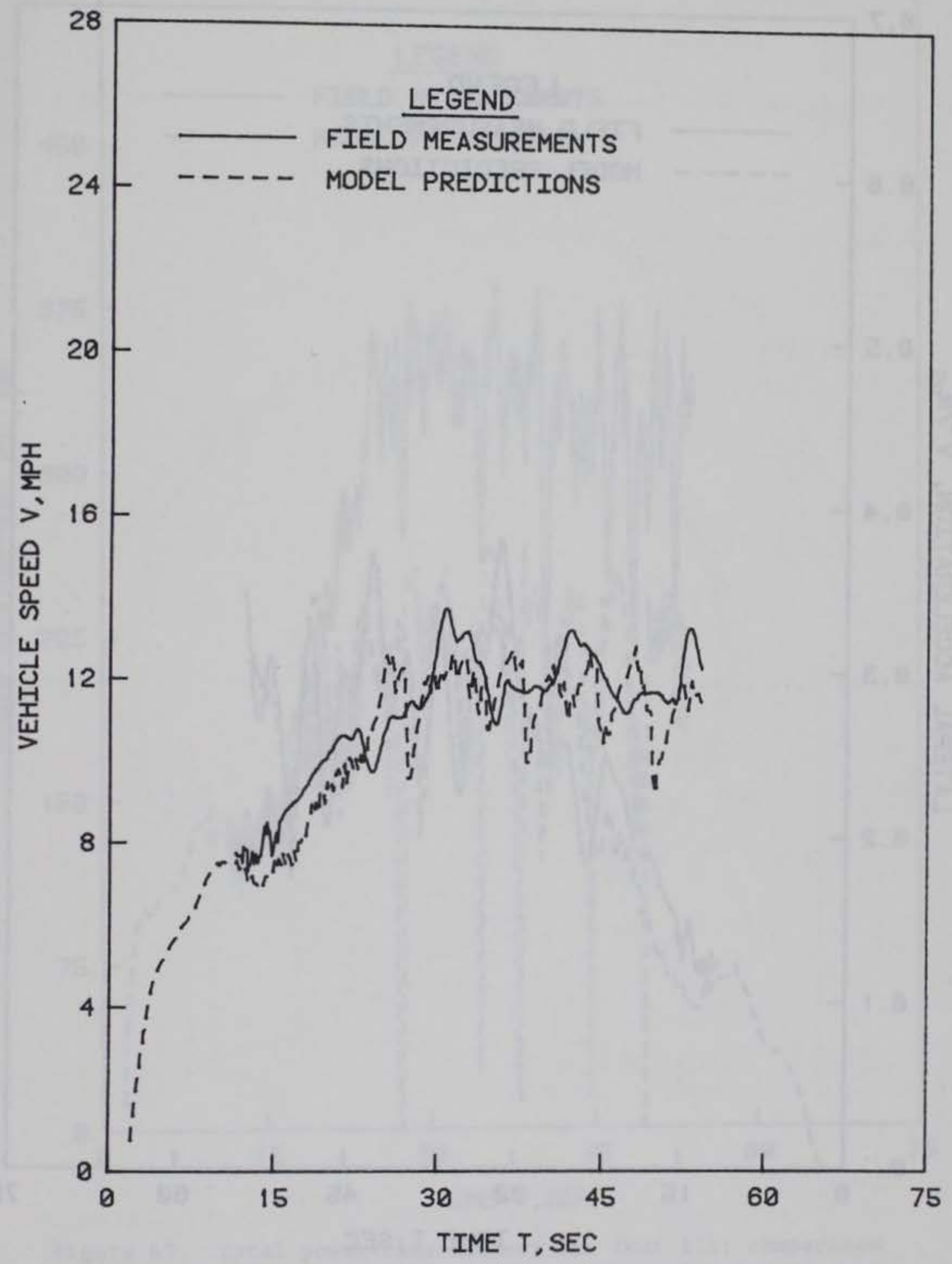

Figure 63. Vehicle speed-time history for test 111; comparison of model predictions with experimental data 


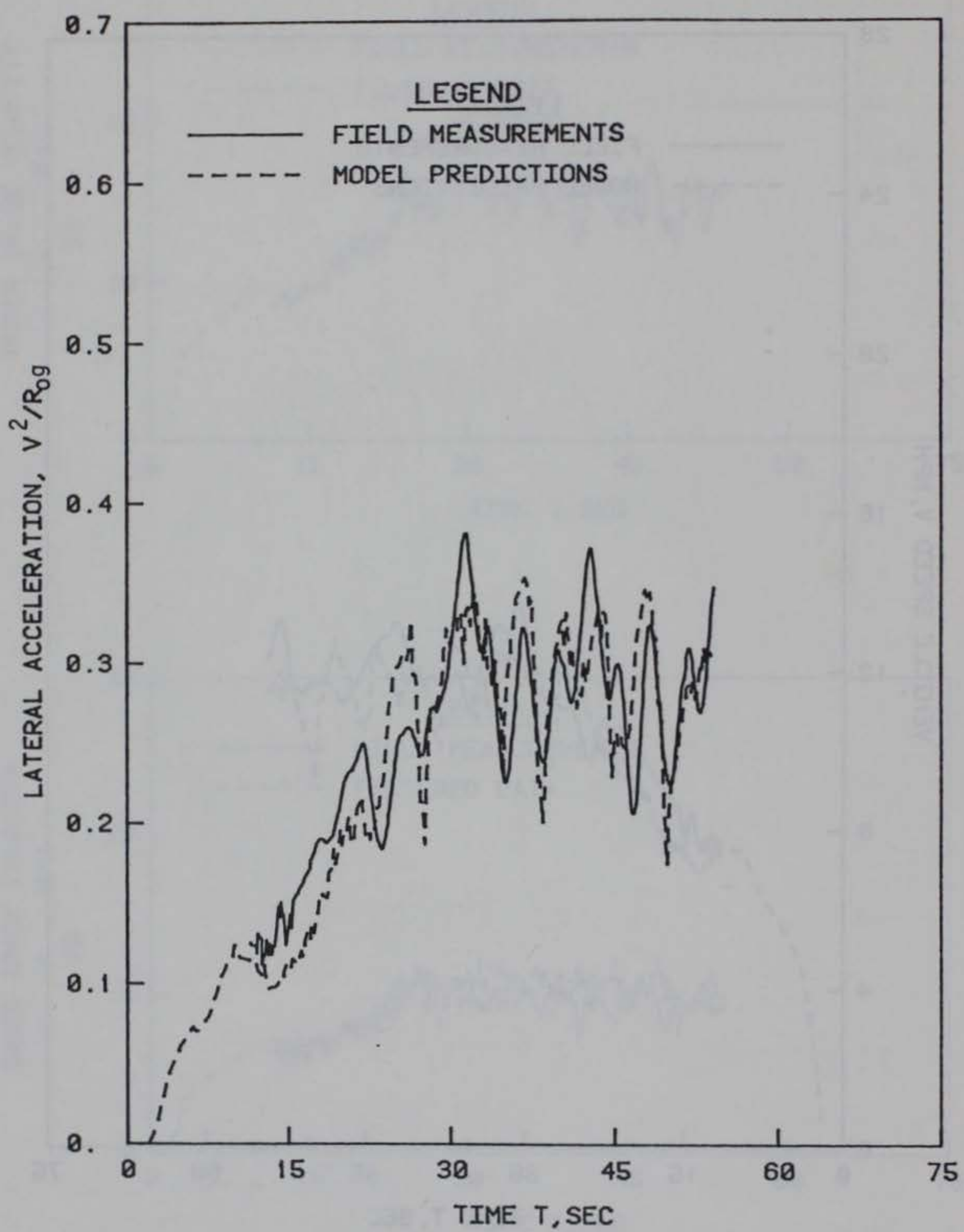

Figure 64. Lateral acceleration-time history for test 111; comparison of model predictions with experimental data 


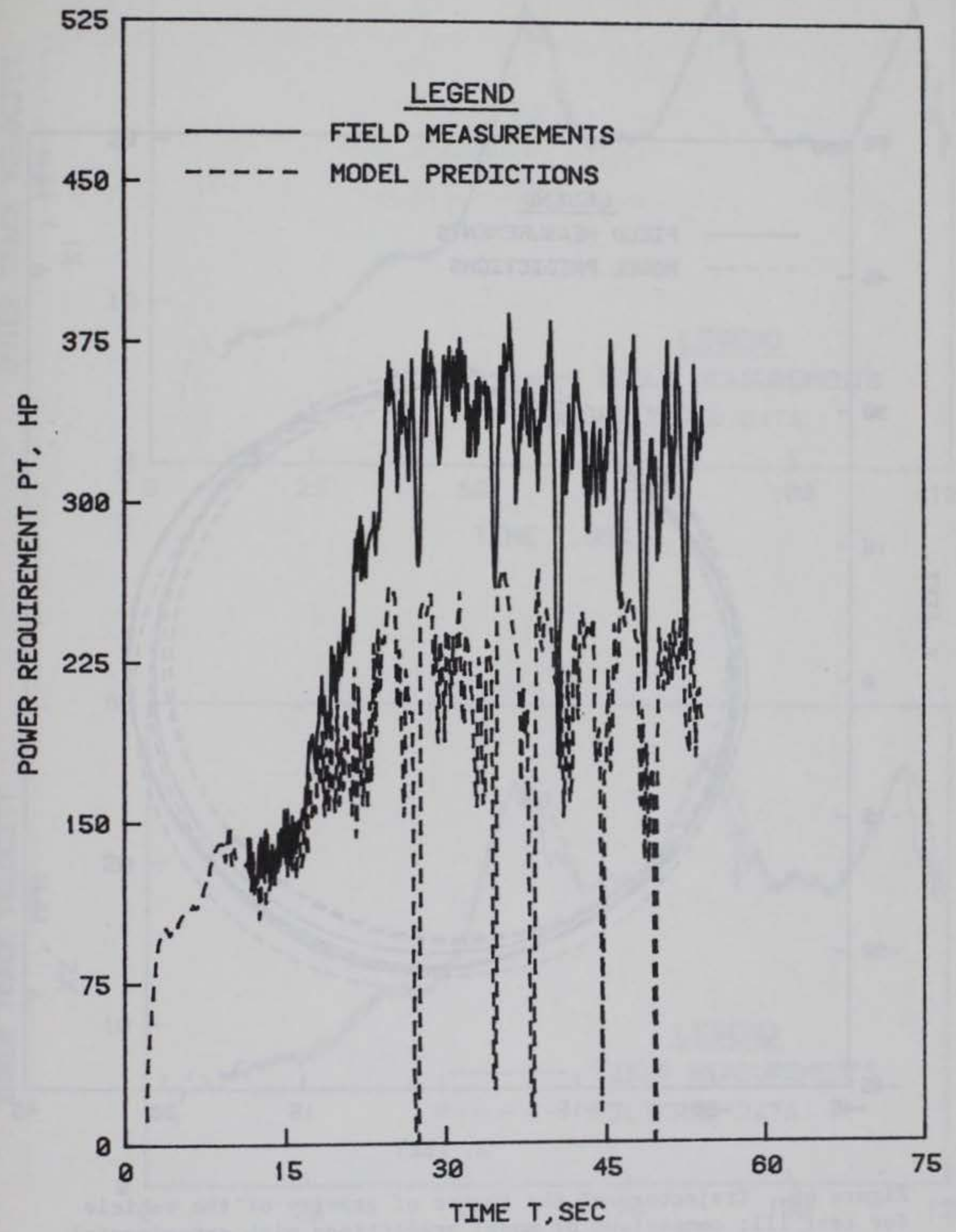

Figure 65. Total power-time history for test 111; comparison of model predictions with experimental data 


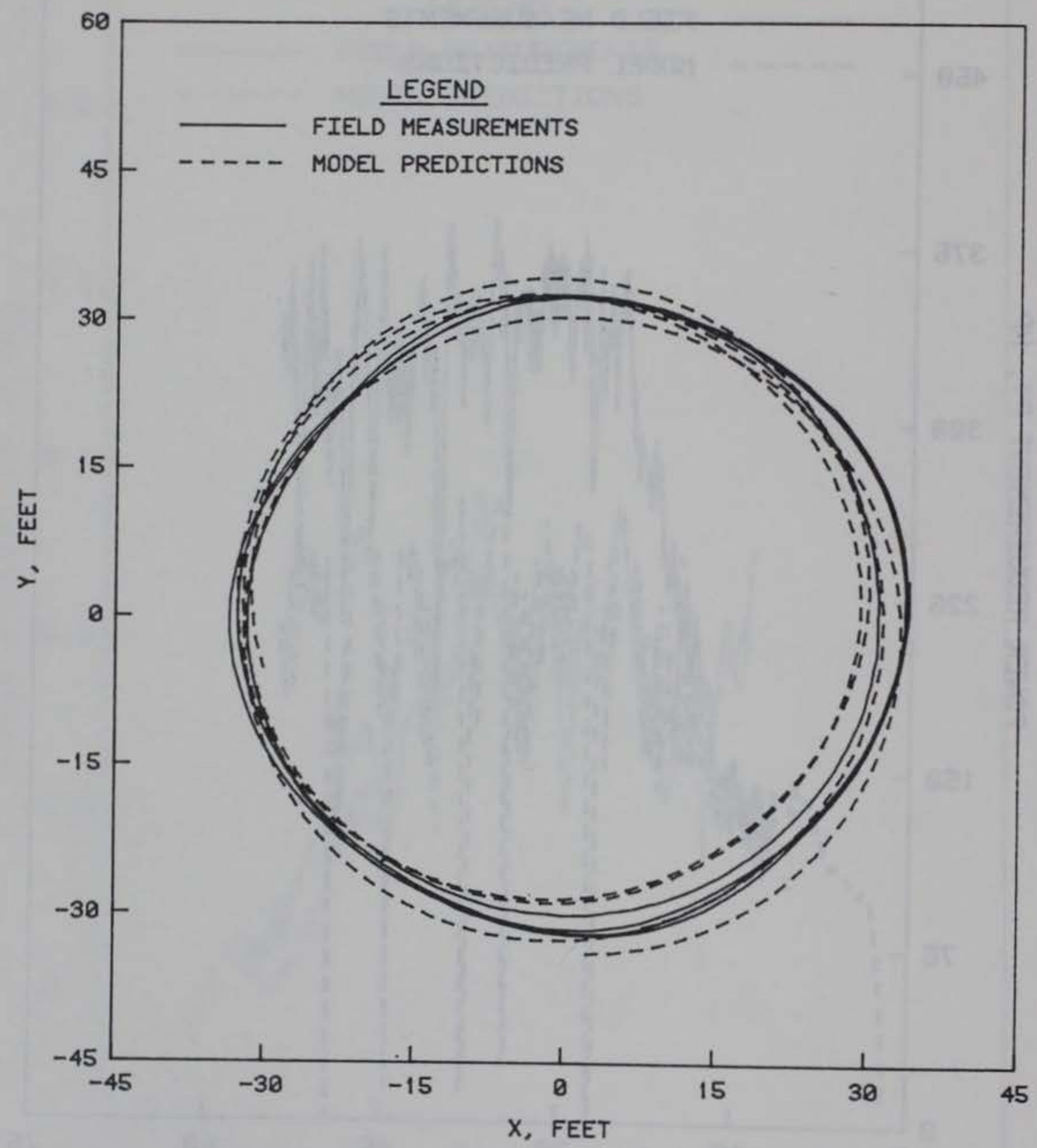

Figure 66. Trajectory of the center of gravity of the vehicle for test 111; comparison of model predictions with experimental data 

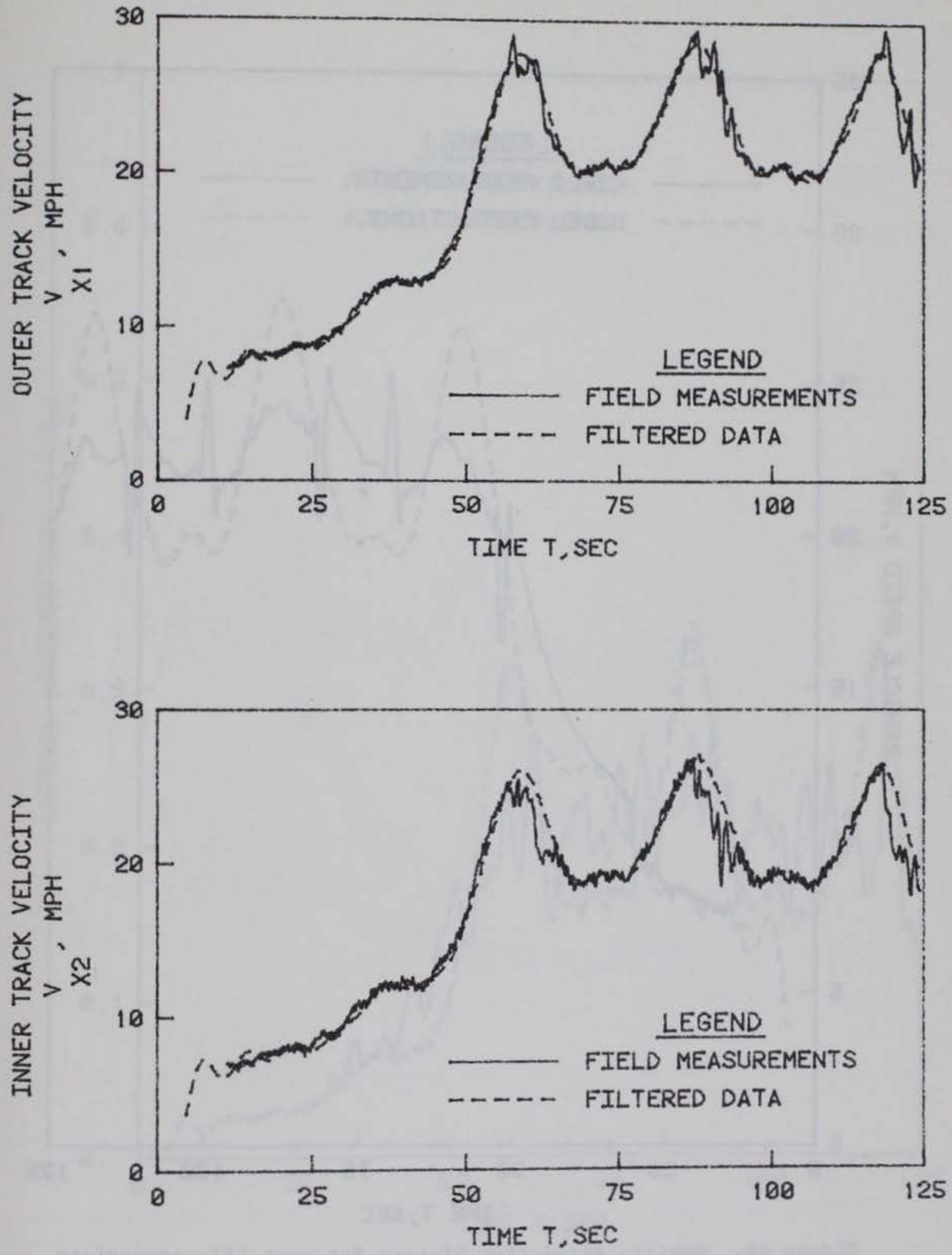

Figure 67. Outer and inner track velocity-time histories for test 117; field measurement and filtered data (input) 


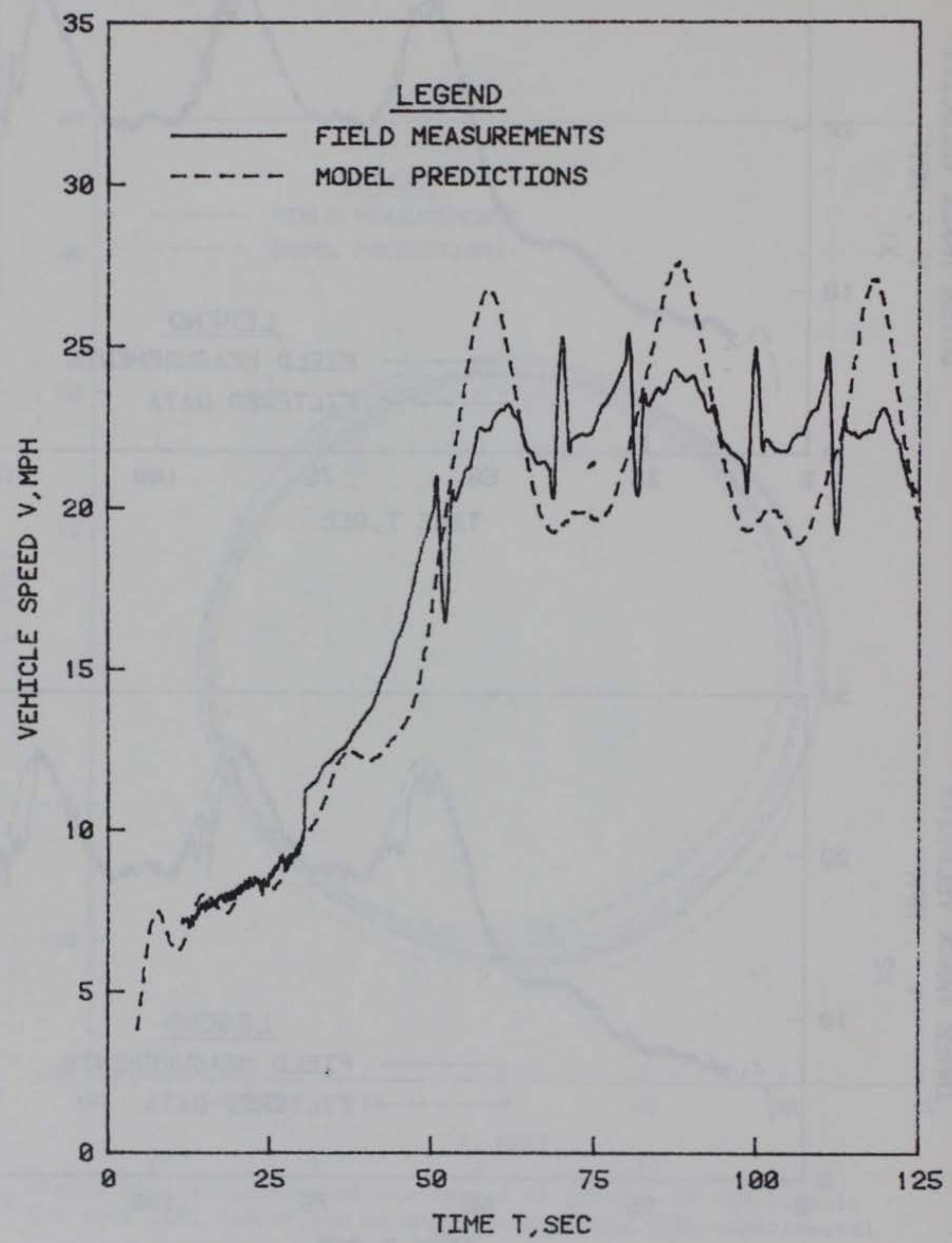

Figure 68. Vehicle speed-time history for test 117; comparison of model predictions with experimental data 


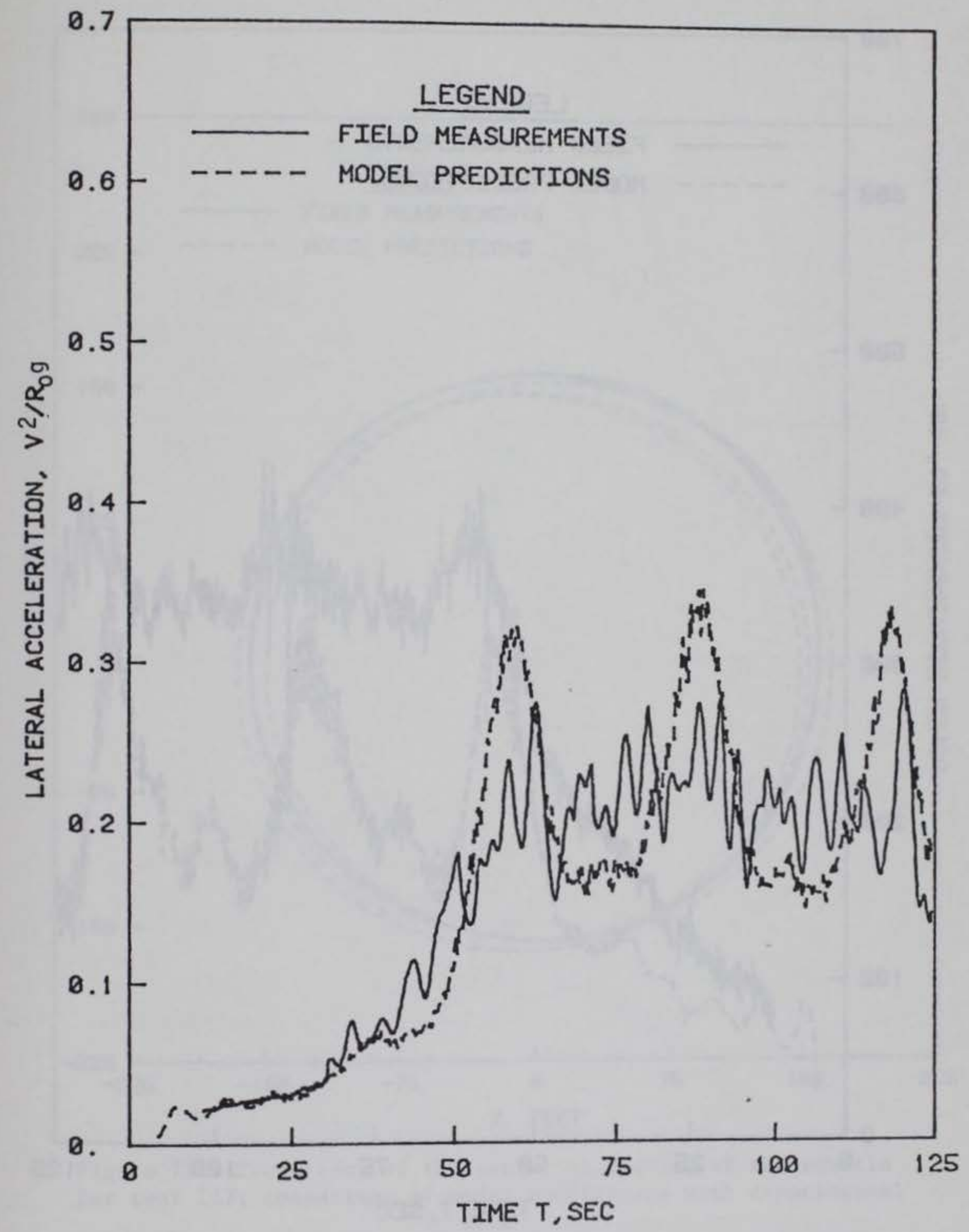

Figure 69. Lateral acceleration-time history for test 117; comparison of model predictions with experimental data 


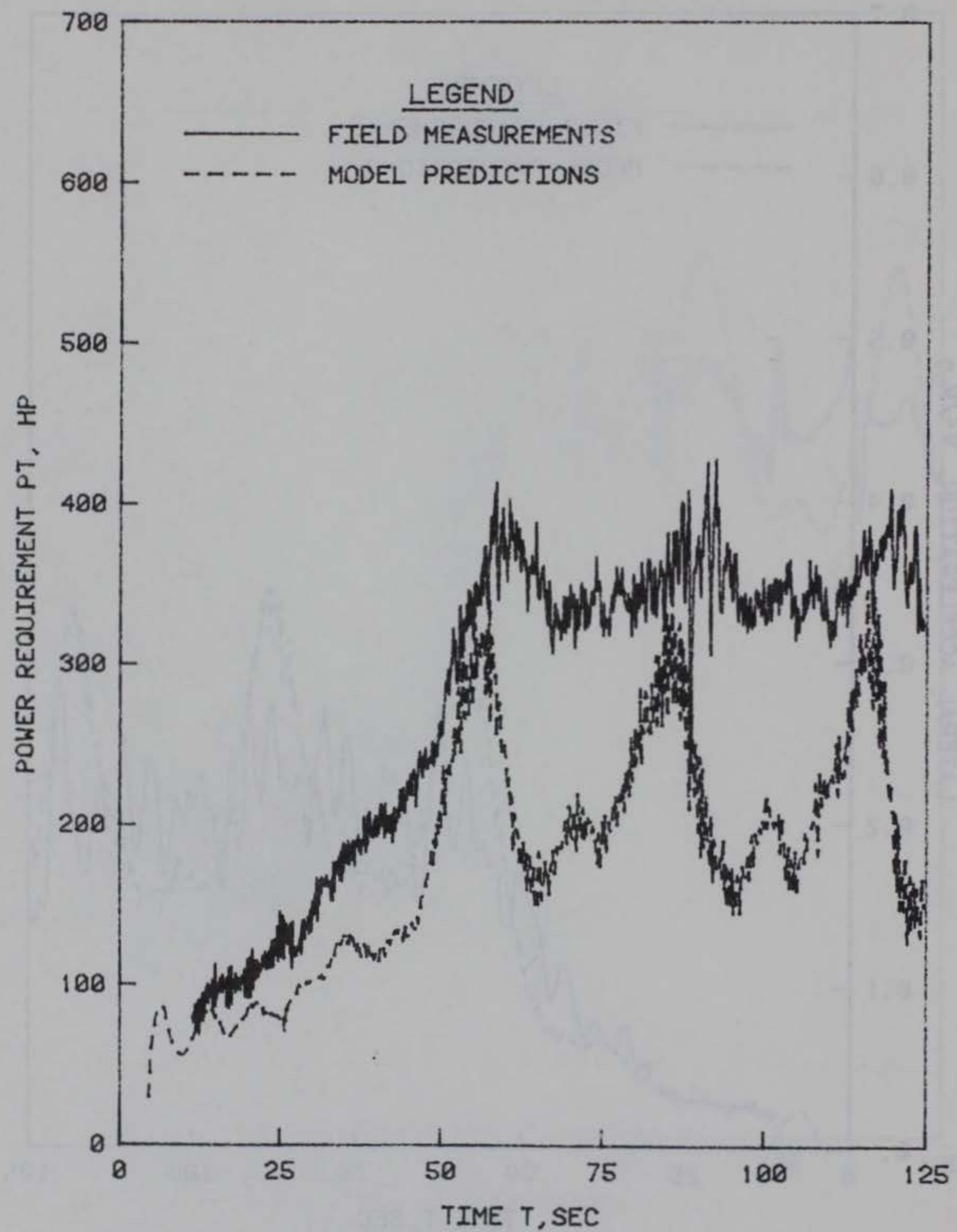

Figure 70. Total power-time history for test 117; comparison of model predictions with experimental data 


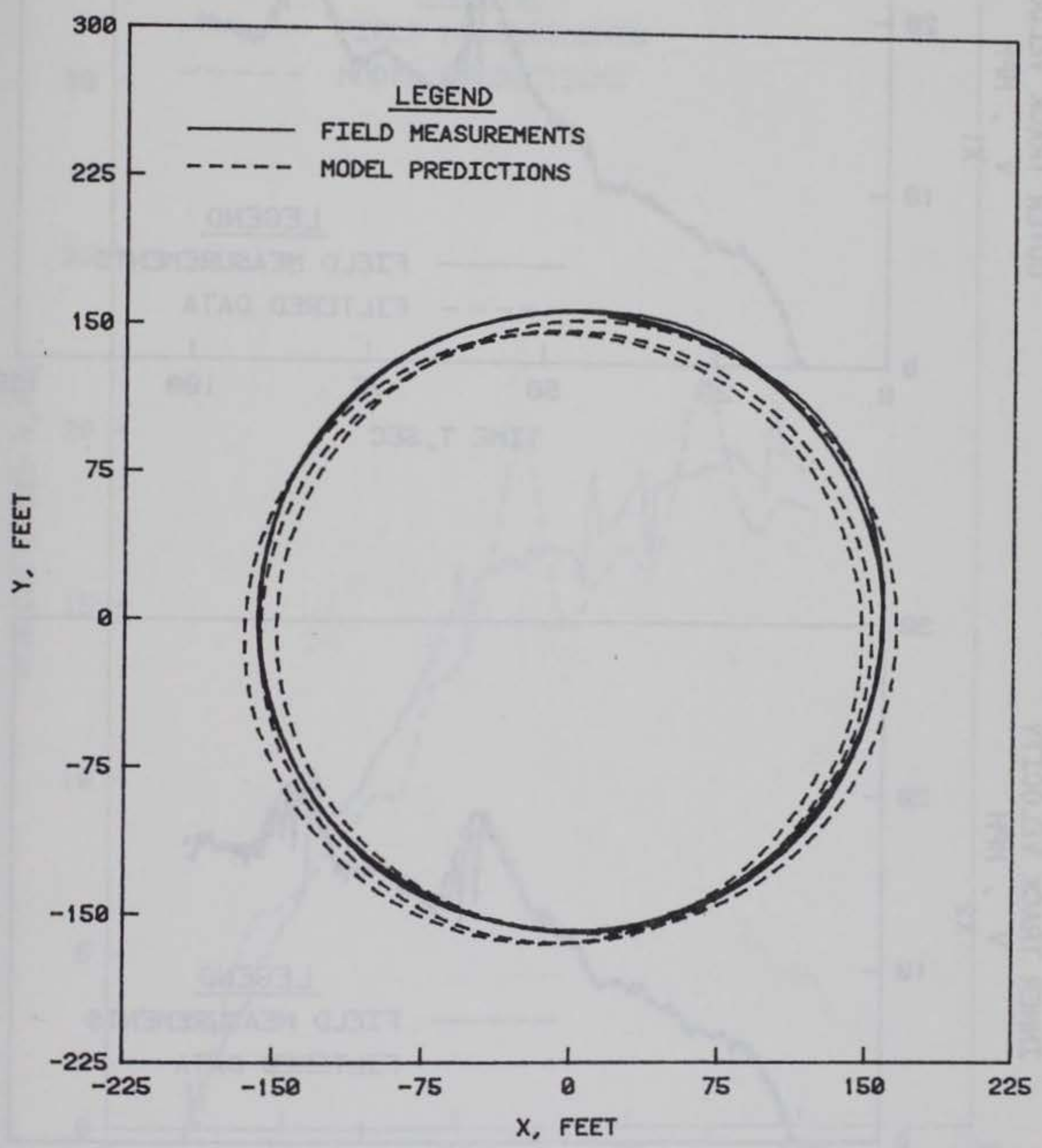

Figure 71. Trajectory of the center of gravity of the vehicle for test 117; comparison of model predictions with experimental data 

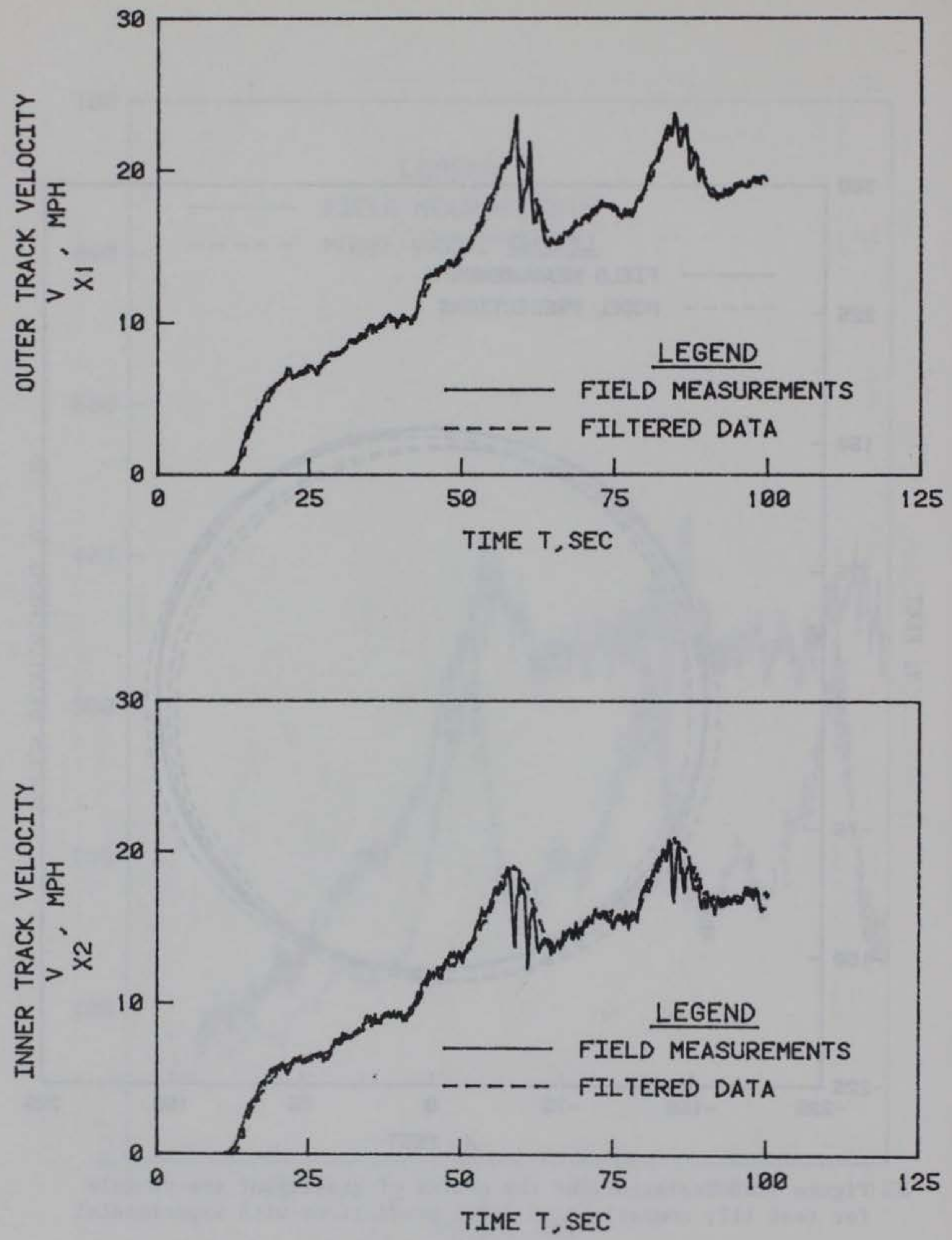

Figure 72. Outer and inner track velocity-time histories for test 118; field measurement and filtered data (input) 


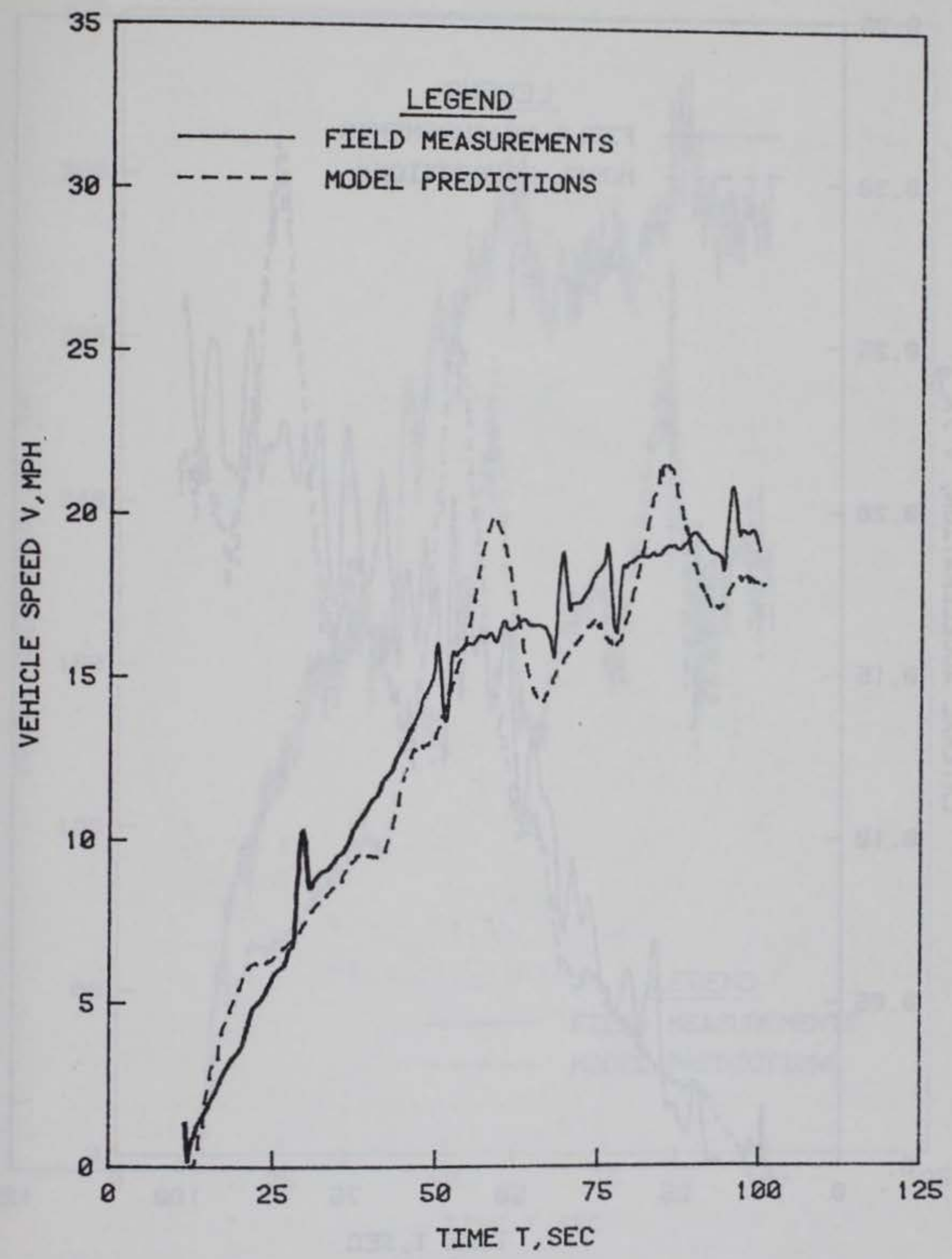

Figure 73. Vehicle speed-time history for test 118; comparison of model predictions with experimental data 


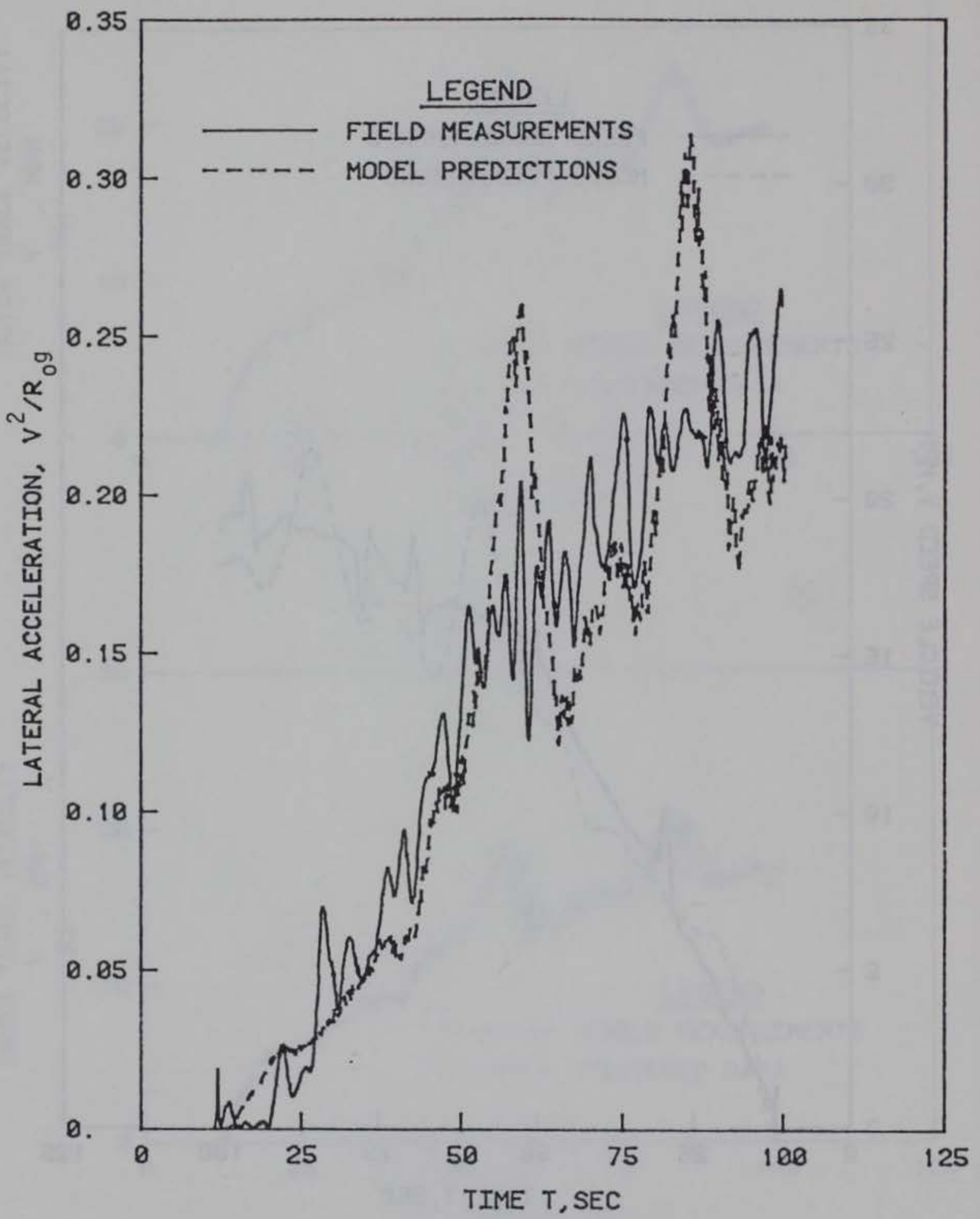

Figure 74. Lateral acceleration-time history for test 118; comparison of model predictions with experimental data 


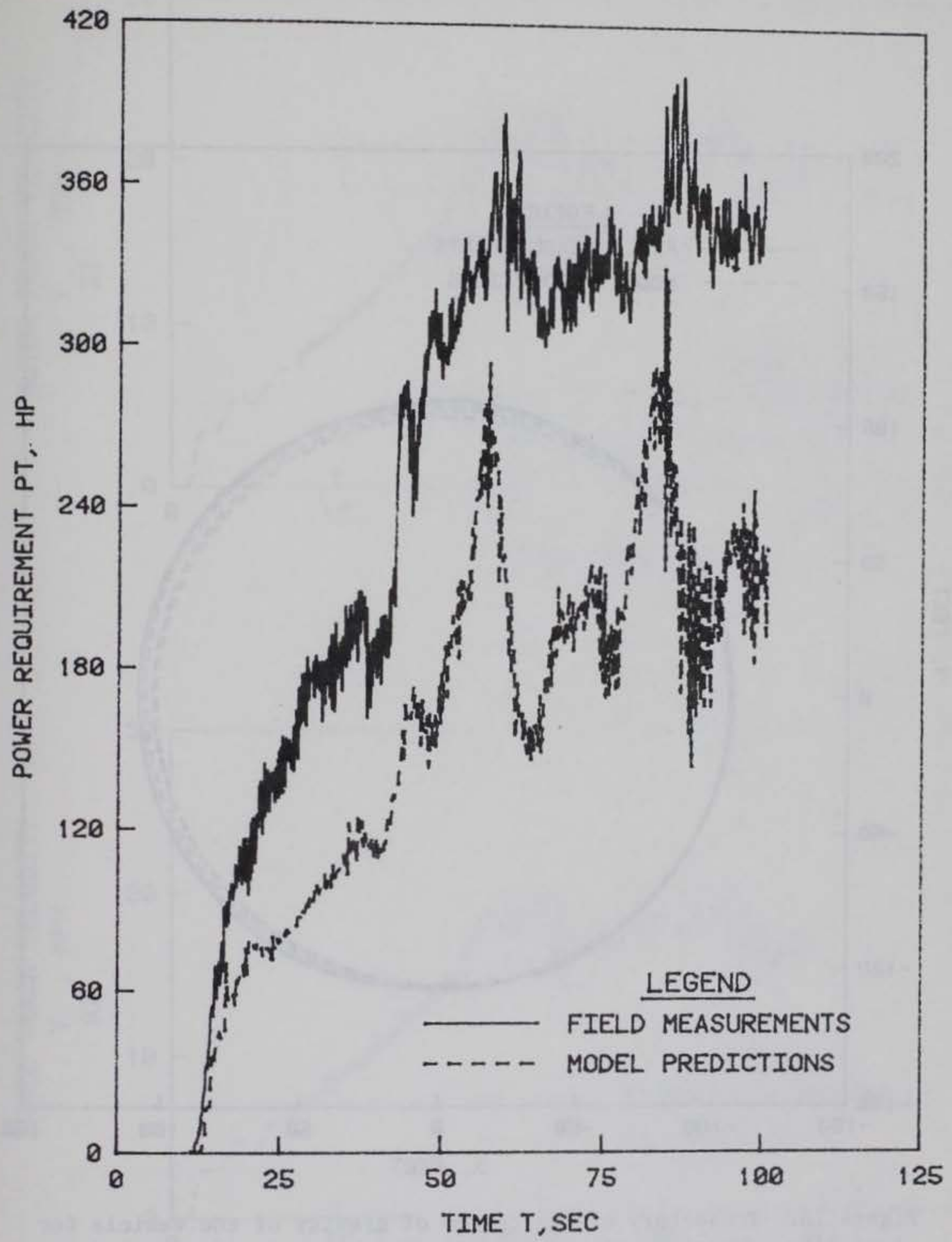

Figure 75. Total power-time history for test 118; comparison of model predictions with experimental data 


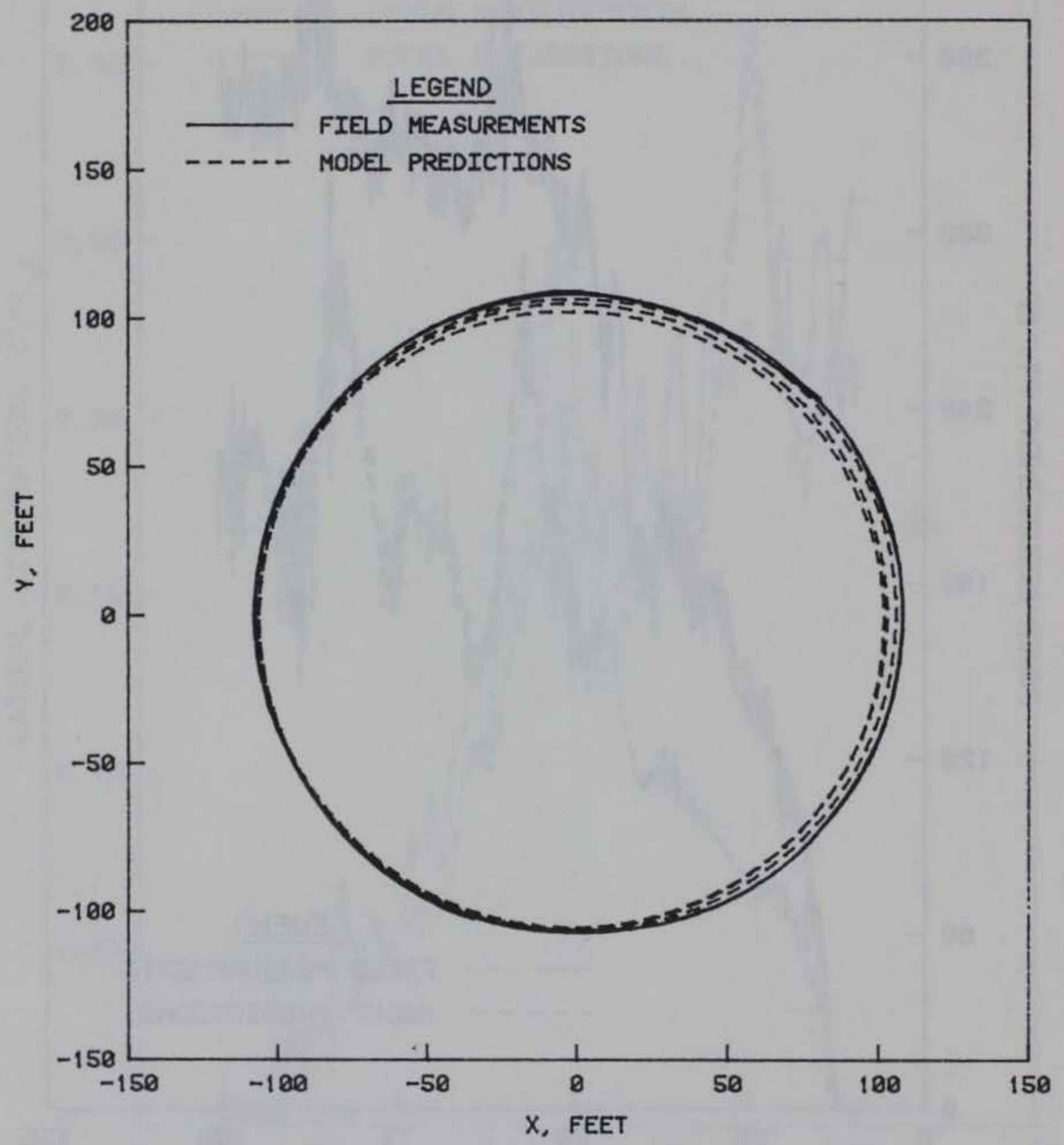

Figure 76. Trajectory of the center of gravity of the vehicle for test 118; comparison of model predictions with experimental data 

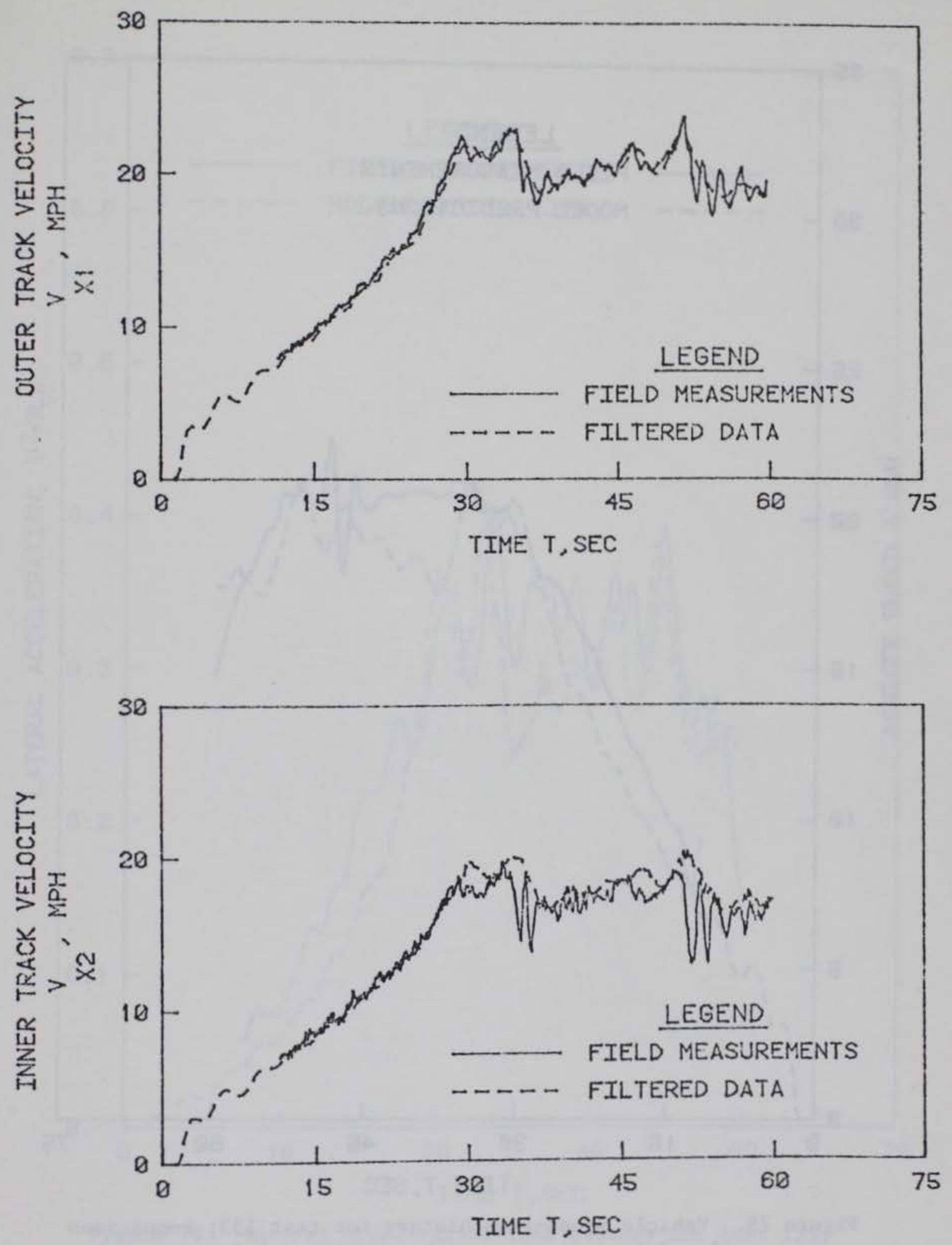

Figure 77. Outer and inner track velocity-time histories for test 133; field measurement and filtered data (input) 


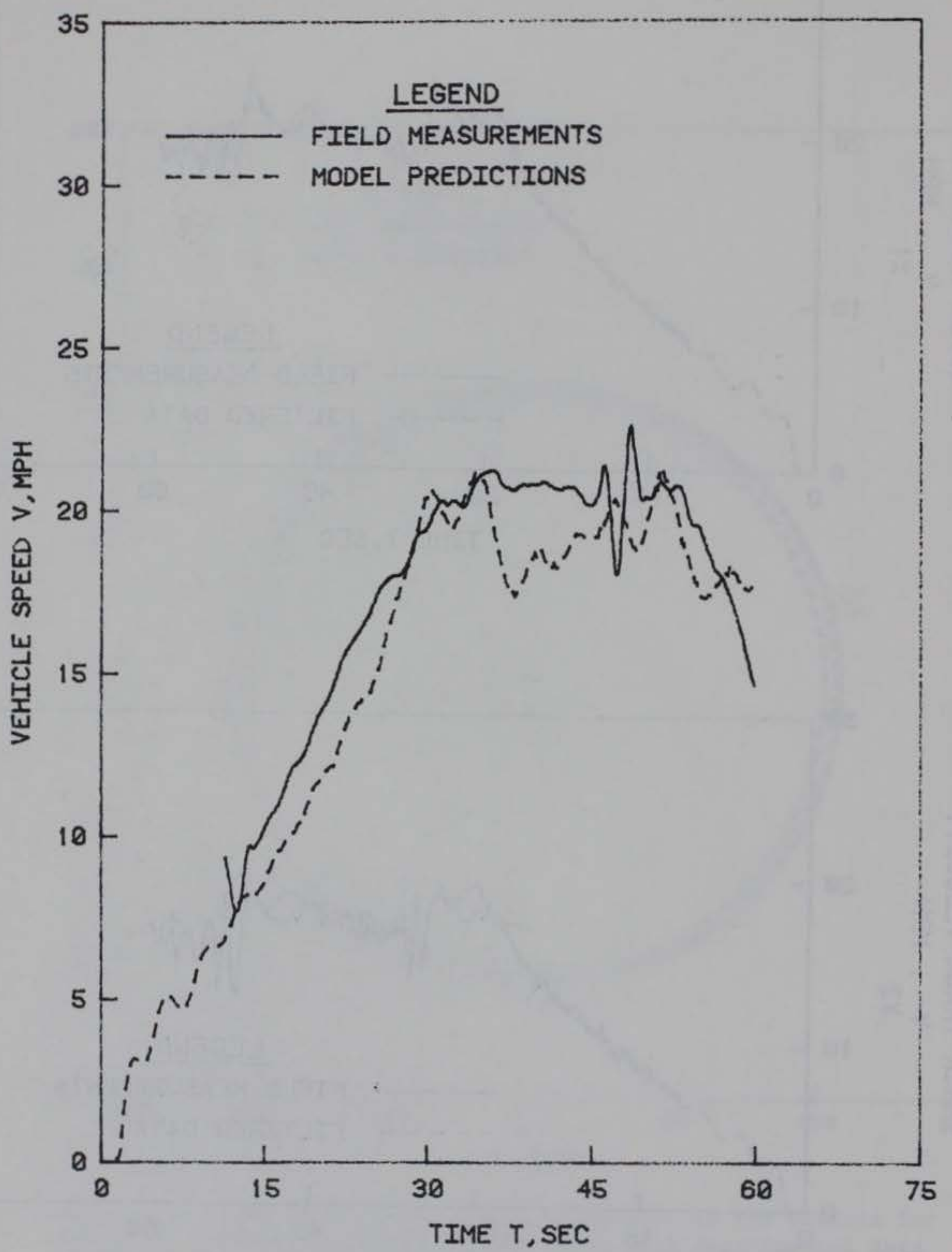

Figure 78. Vehicle speed-time history for test 133; comparison of model predictions with experimental data 


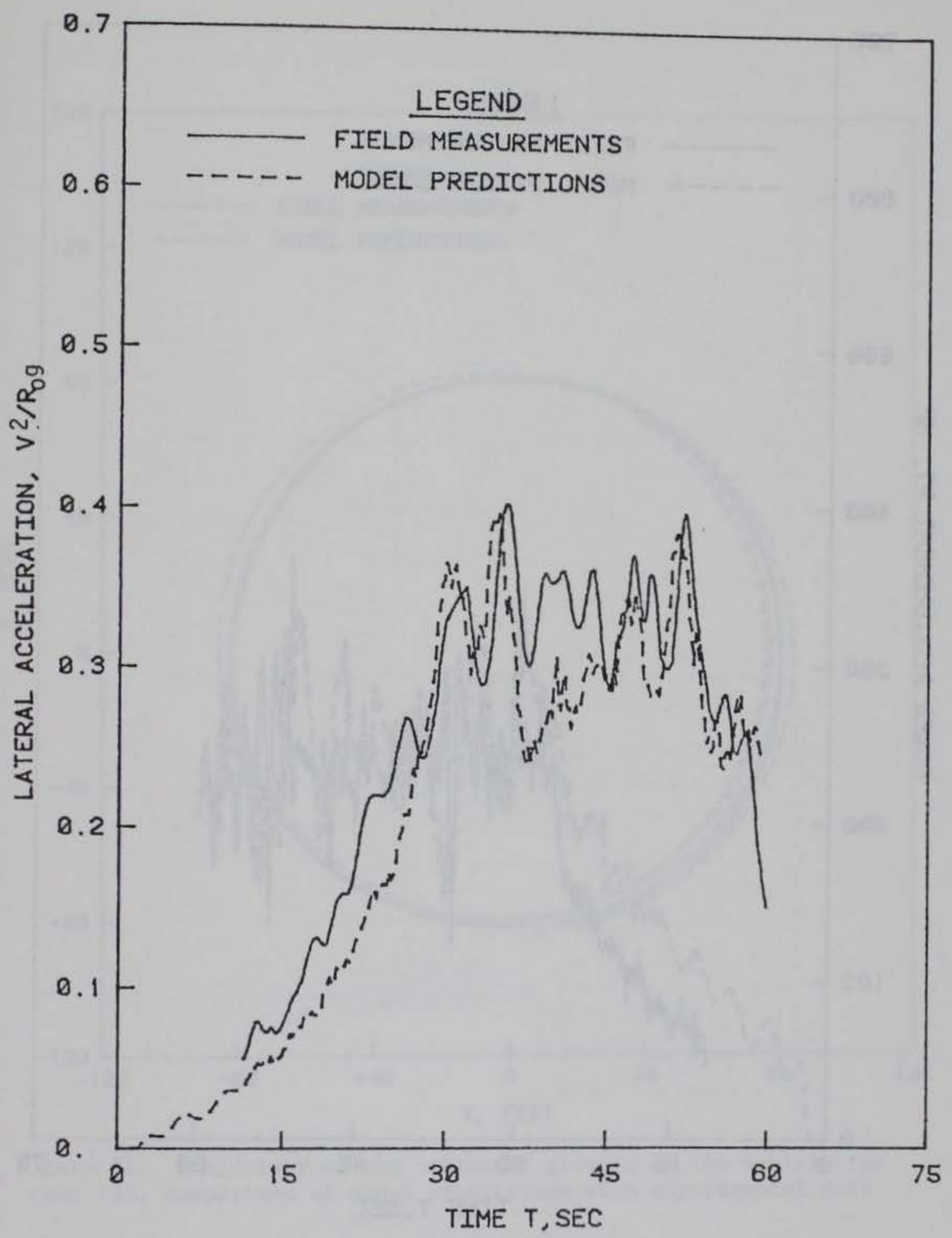

Figure 79. Lateral acceleration-time history for test 133; comparison of model predictions with experimental data 


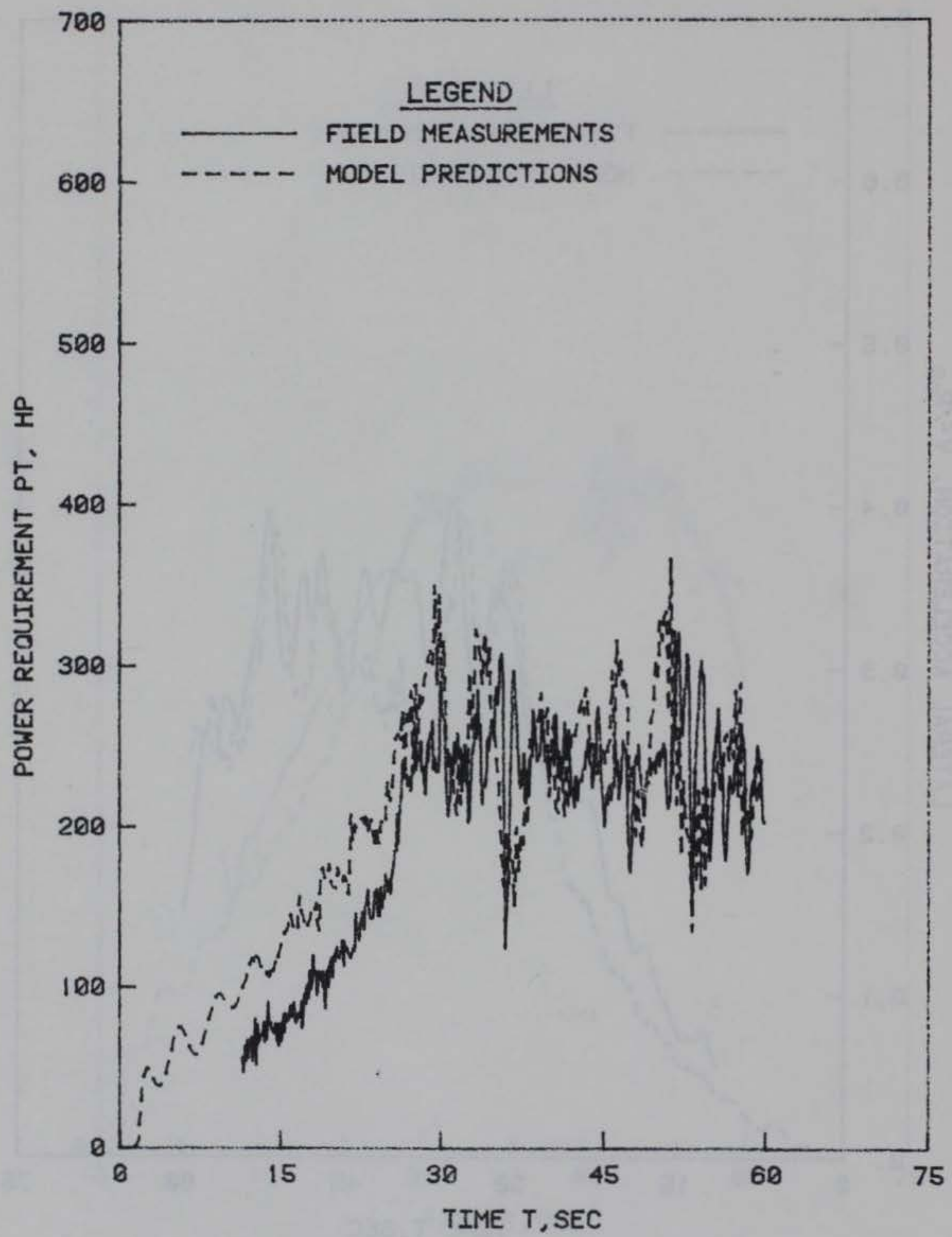

Figure 80. Total power-time history for test 133; comparison of model predictions with experimental data 


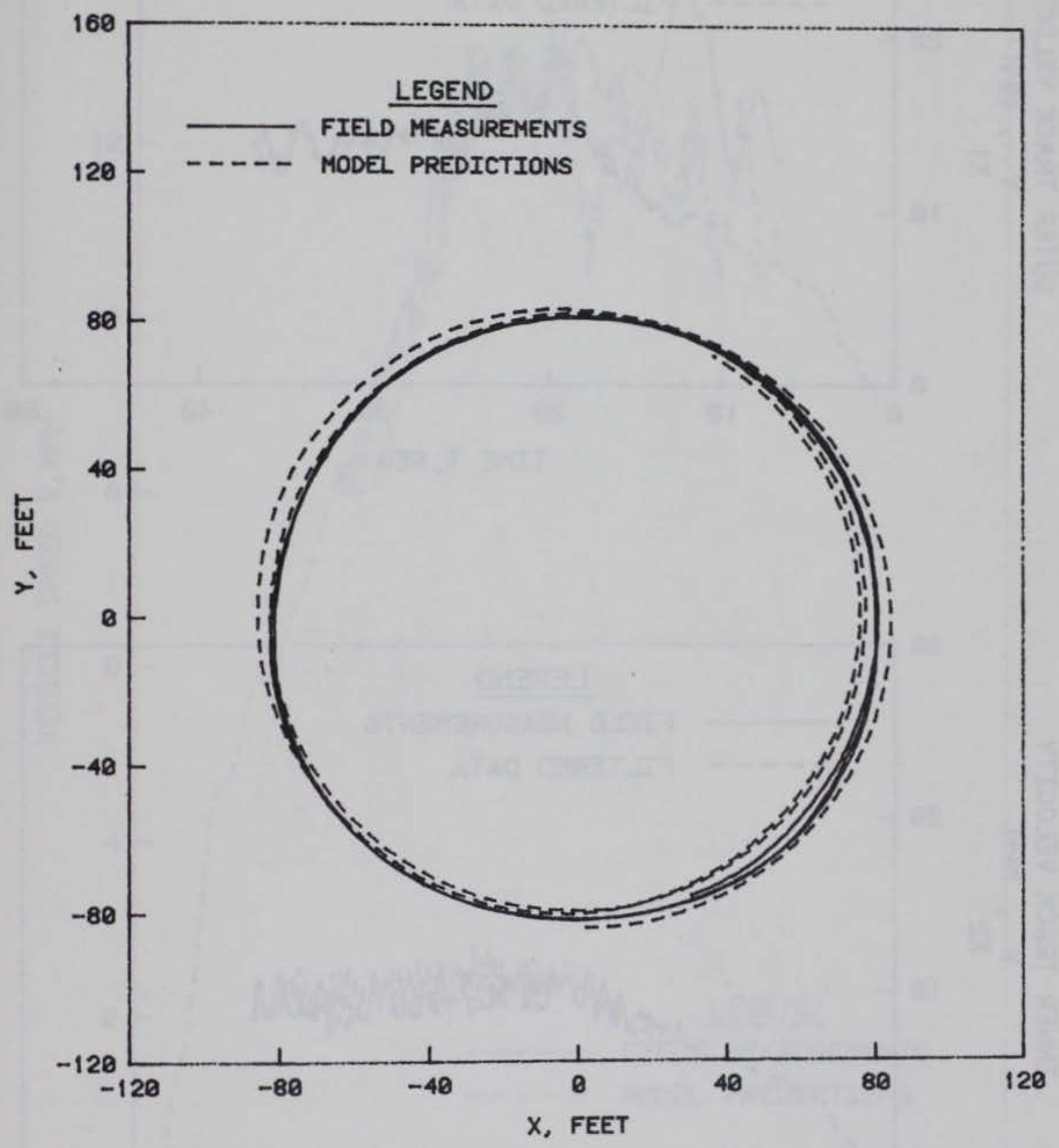

Figure 81. Trajectory of the center of gravity of the vehicle for test 133; comparison of model predictions with experimental data 

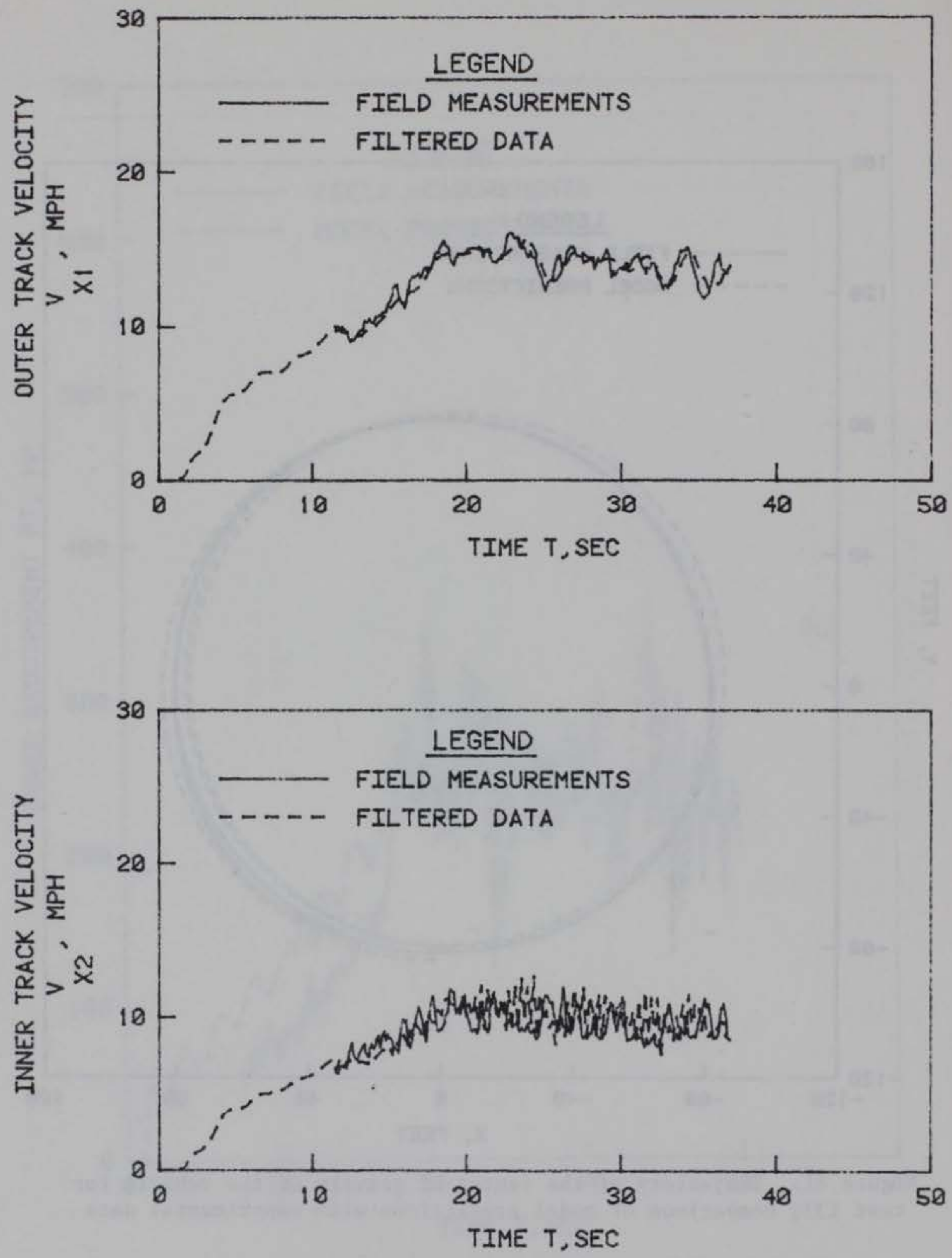

Figure 82. Outer and inner track velocity-time histories for test 134; field measurement and filtered data (input) 


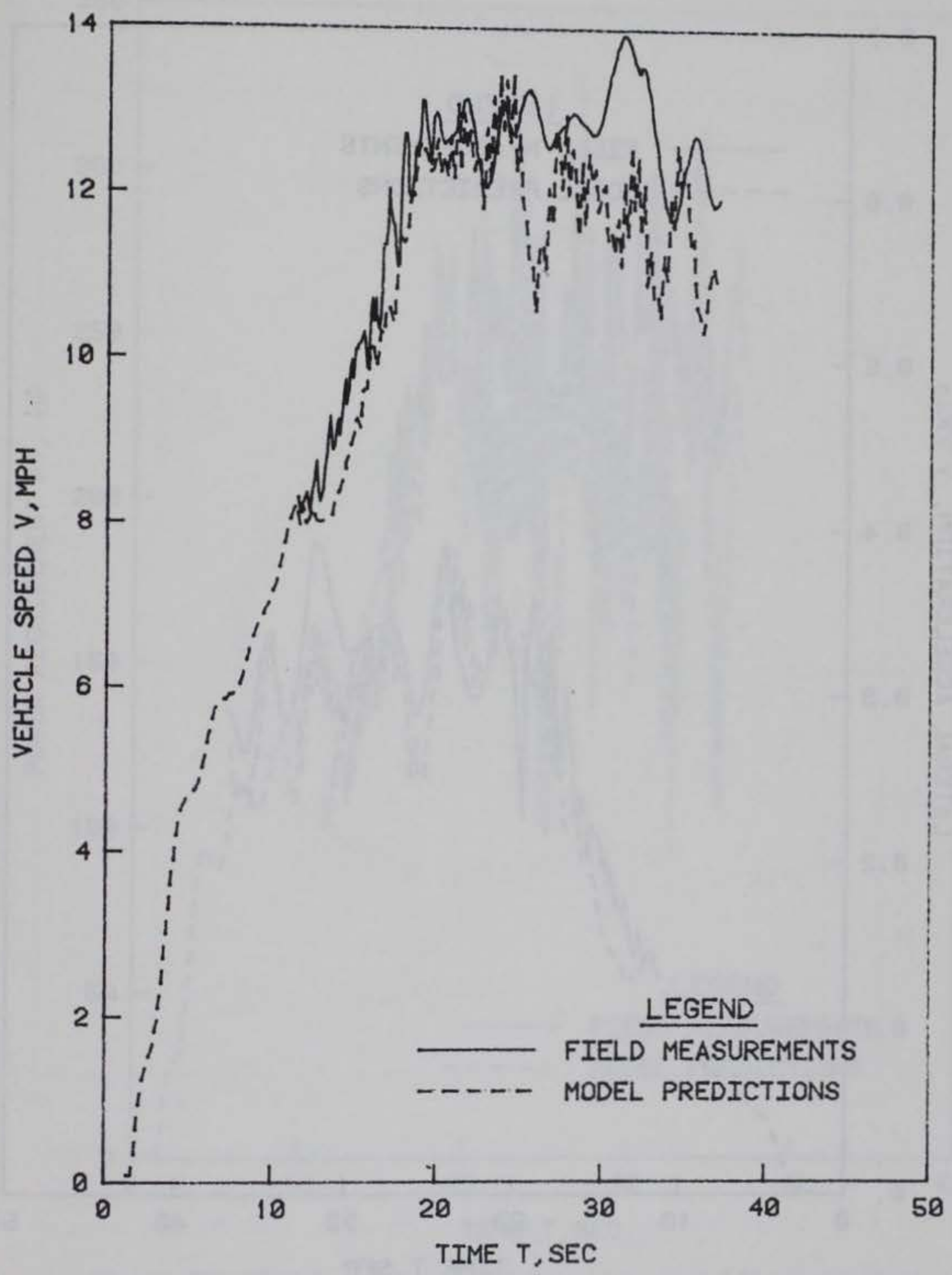

Figure 83. Vehicle speed-time history for test 134; comparison of model predictions with experimental data 


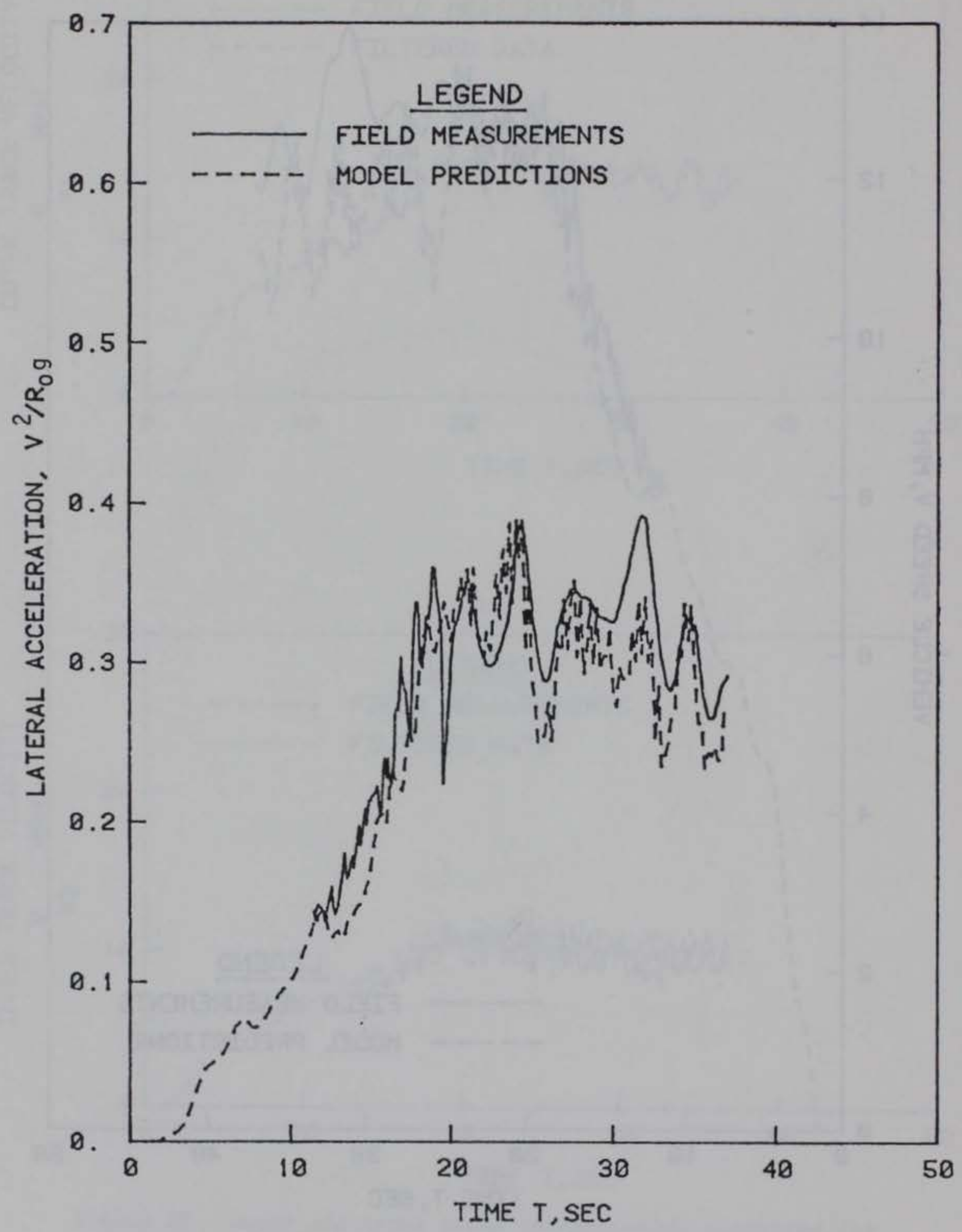

Figure 84. Lateral acceleration-time history for test 134; comparison of model predictions with experimental data 


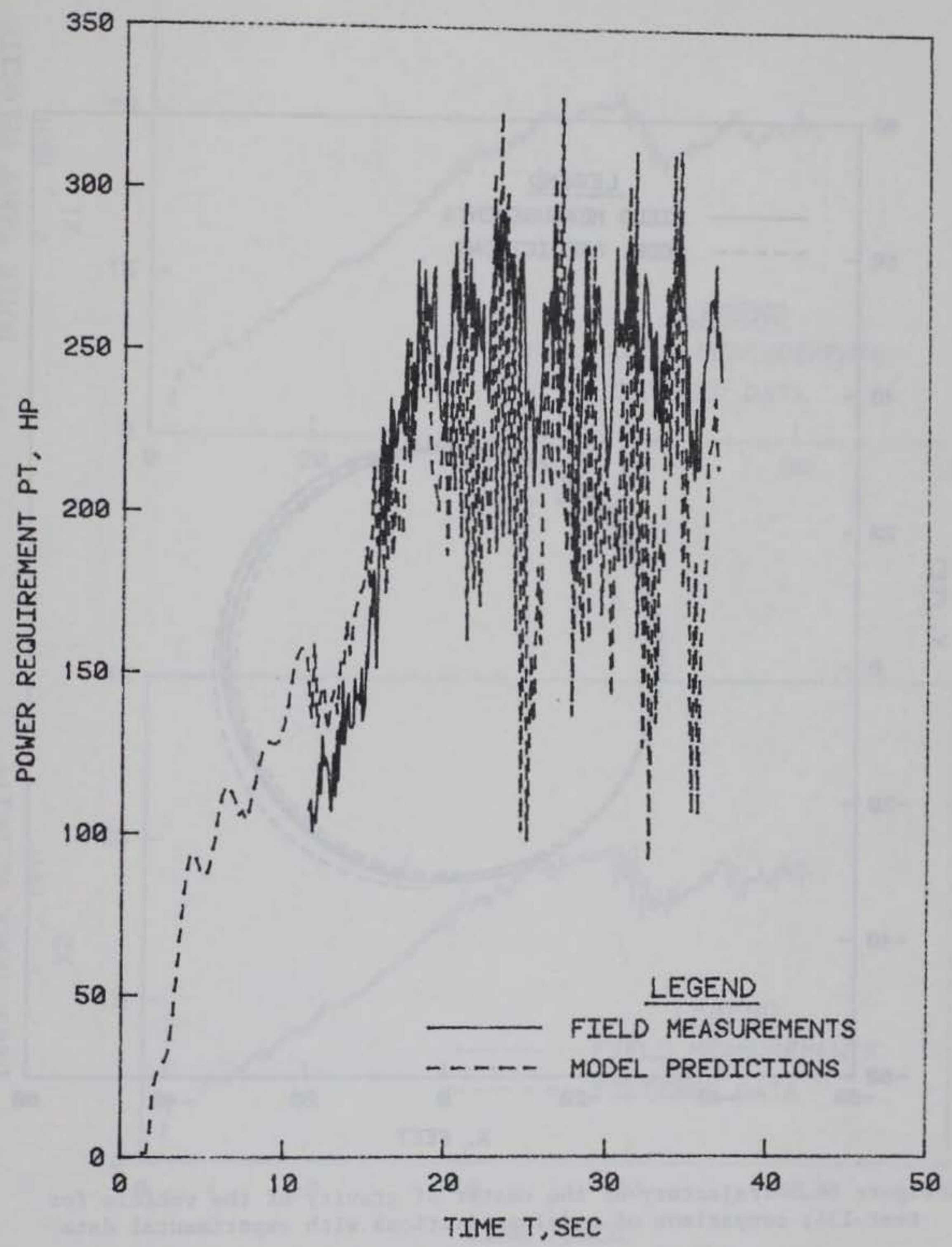

Figure 85. Total power-time history for test 134; comparison of model predictions with experimental data 


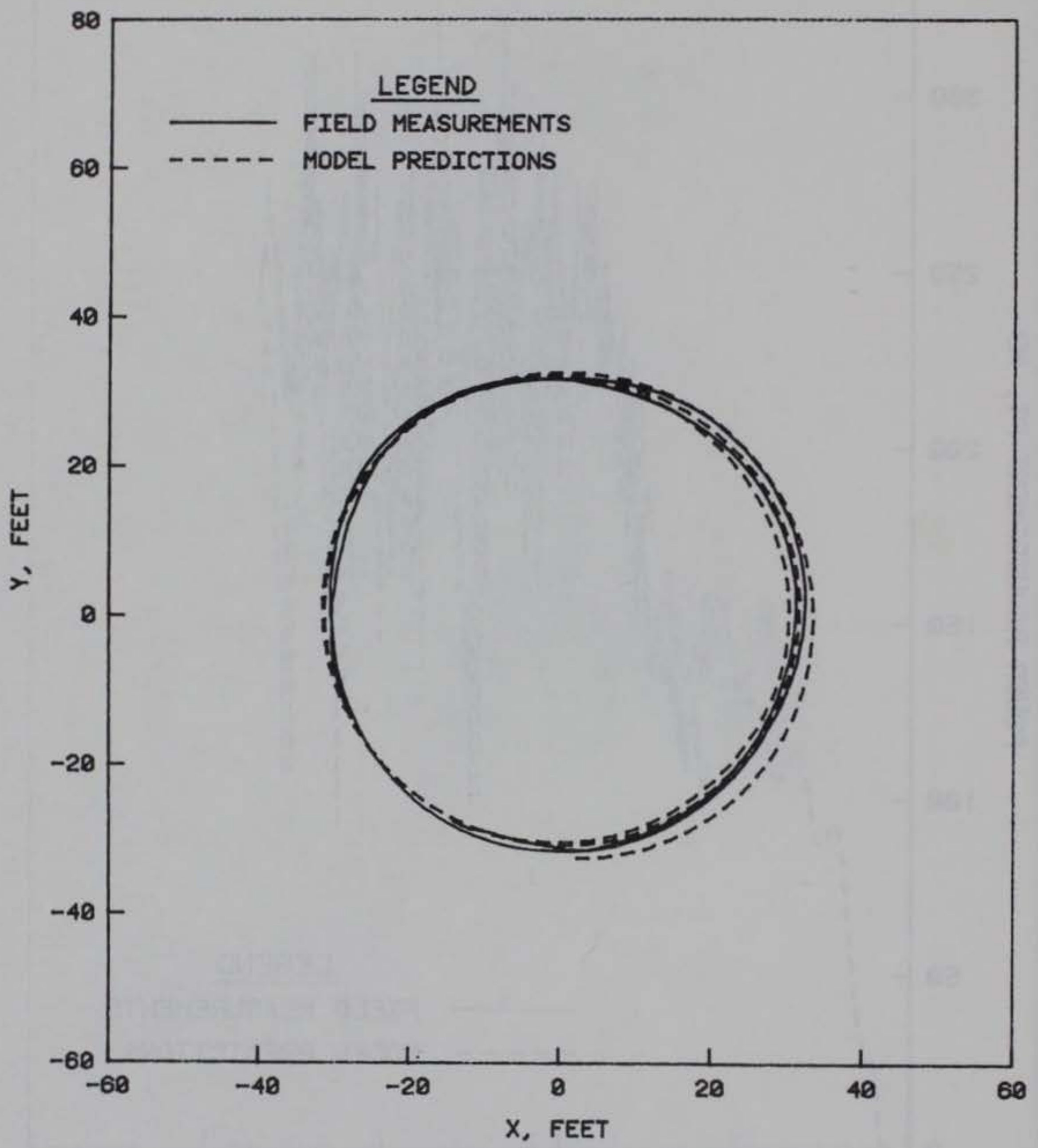

Figure 86. Trajectory of the center of gravity of the vehicle for test 134; comparison of model predictions with experimental data 

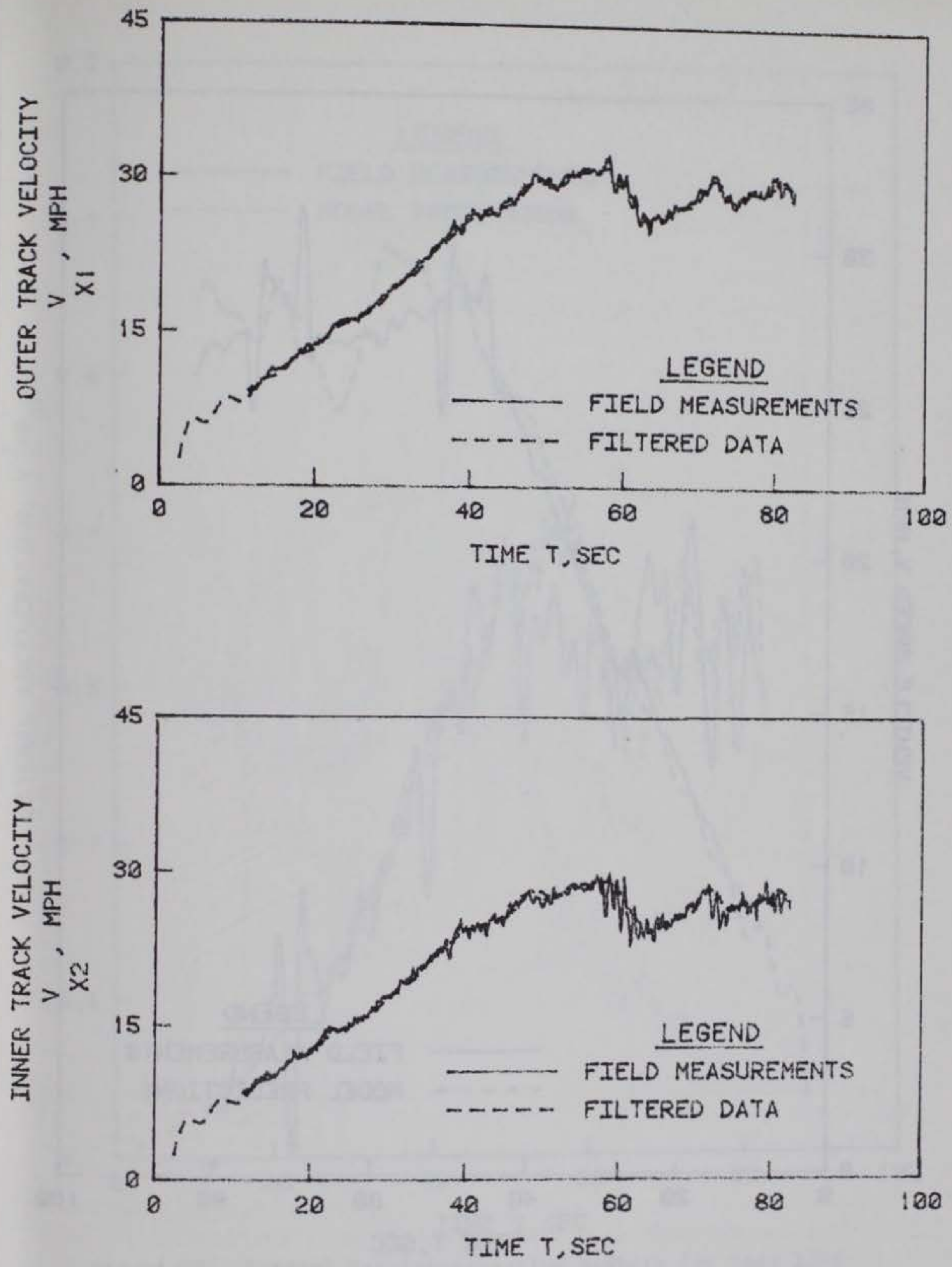

Figure 87. Outer and inner track velocity-time histories for test 139; field measurement and filtered data (input) 


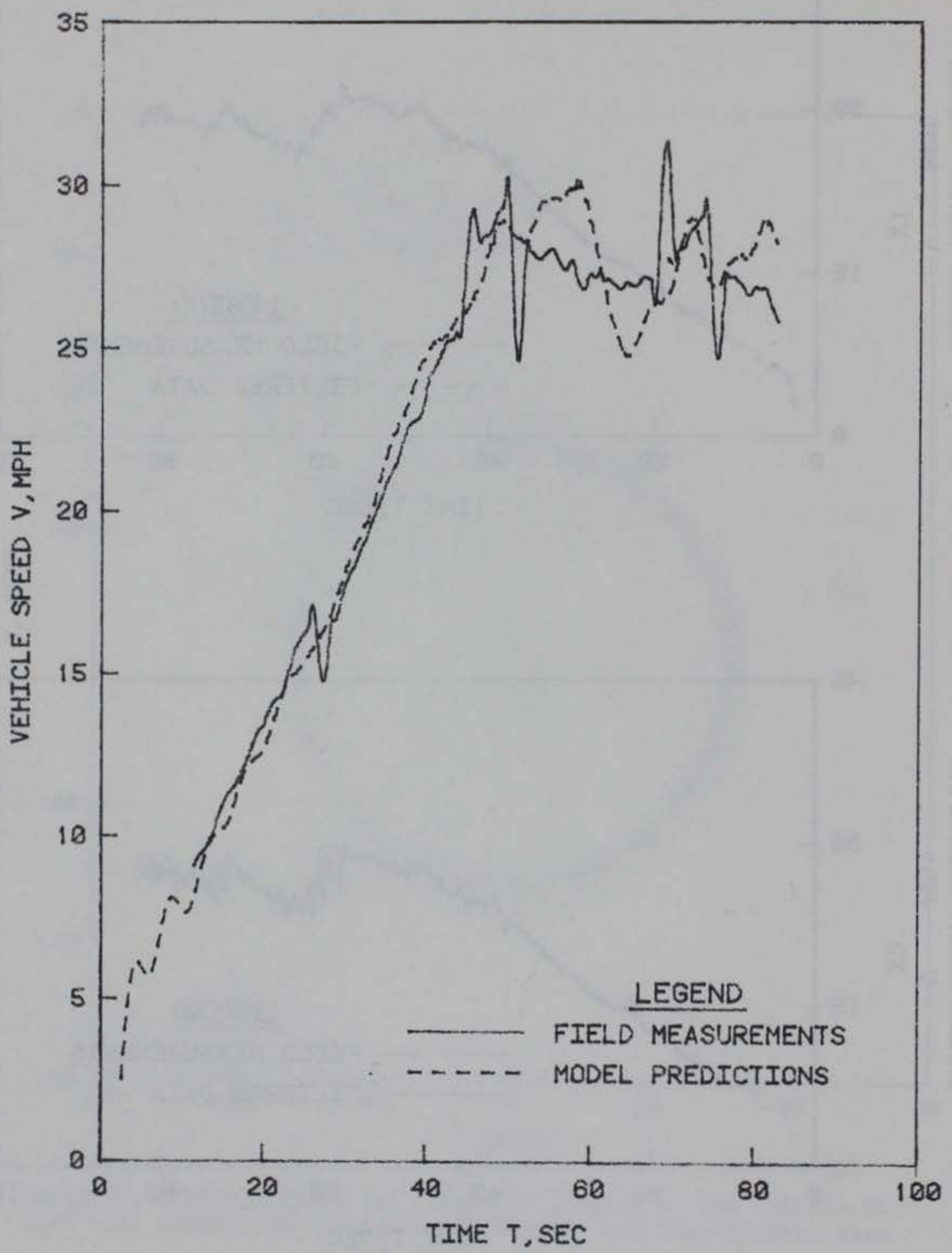

Figure 88. Vehicle speed-time history for test 139; comparison of model predictions with experimental data 


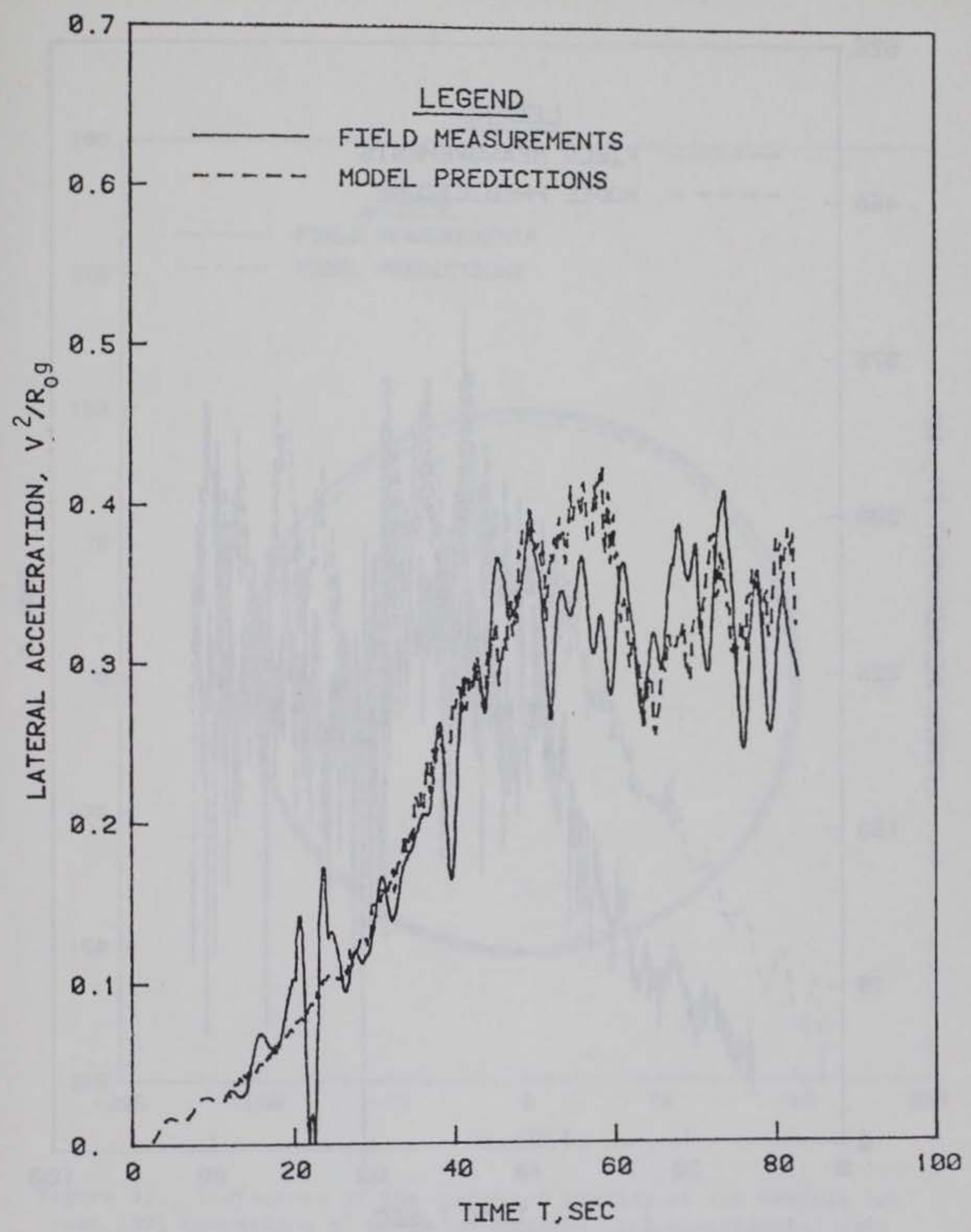

Figure 89. Lateral acceleration-time history for test 139; comparison of model predictions with experimental data 


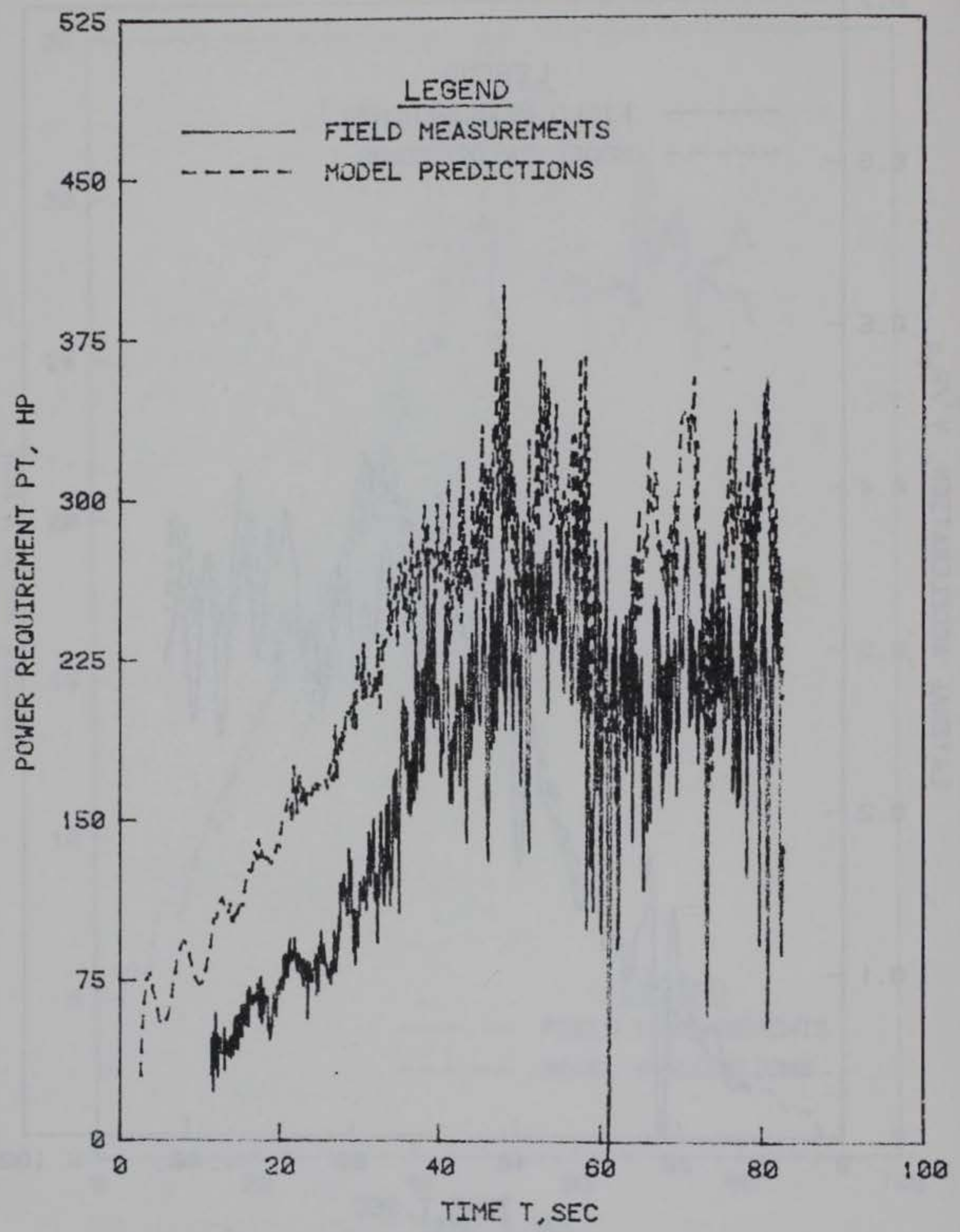

Figure 90. Total power-time history for test 139; comparison of model predictions with experimental data 


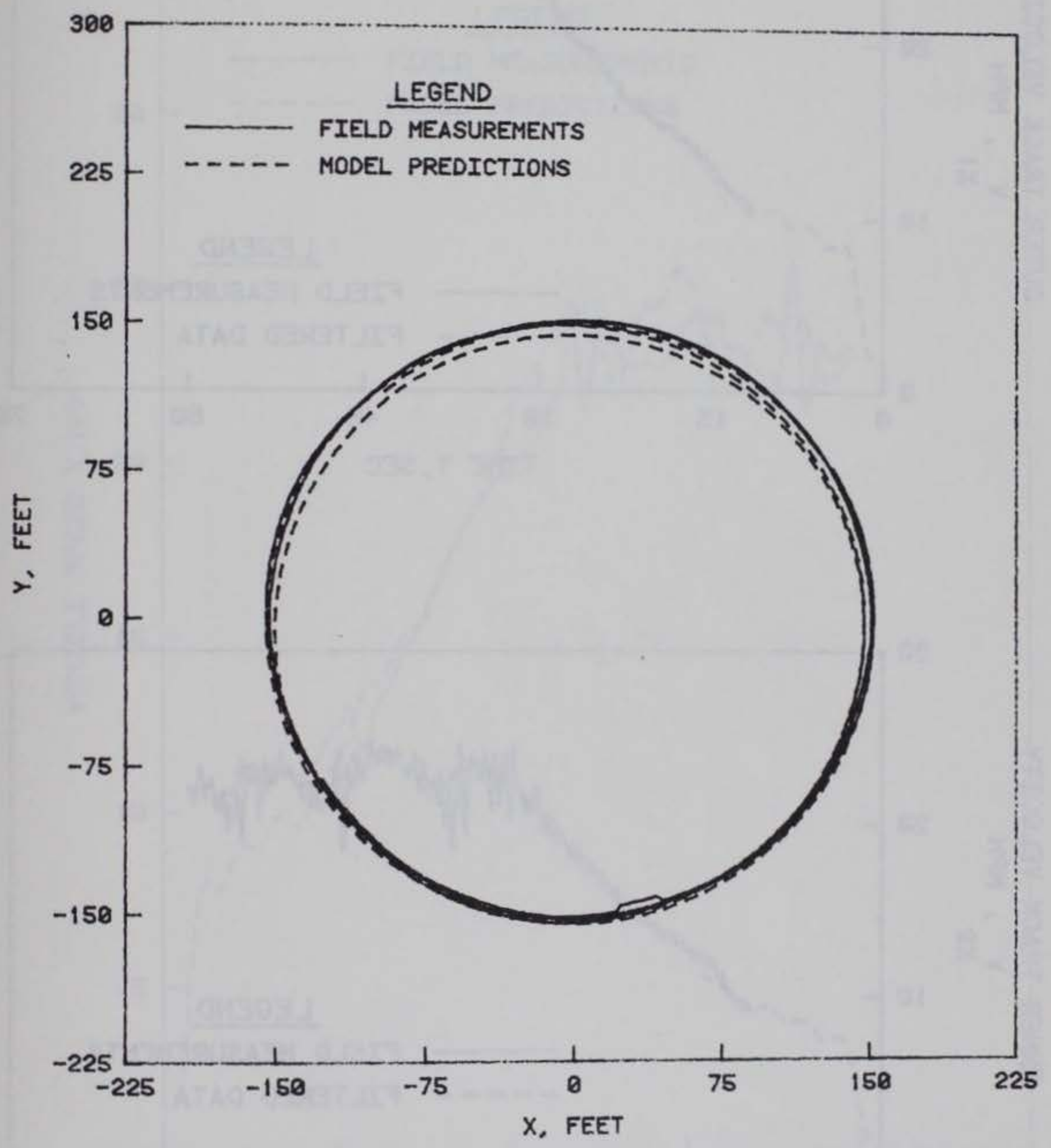

Figure 91. Trajectory of the center of gravity of the vehicle for test 139; comparison of model predictions with experimental data 

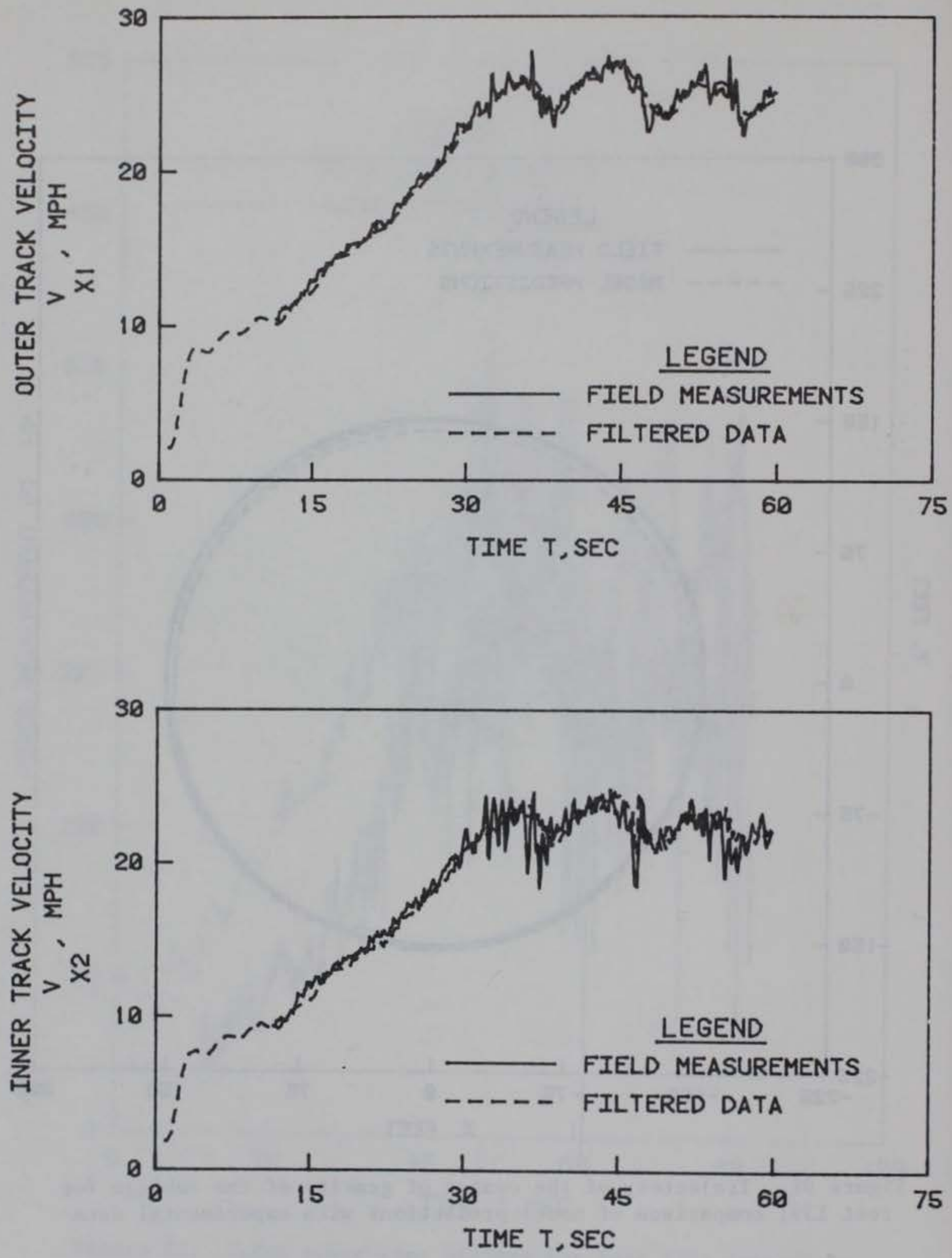

Figure 92. Outer and inner track velocity-time histories for test 140; field measurement and filtered data (input) 


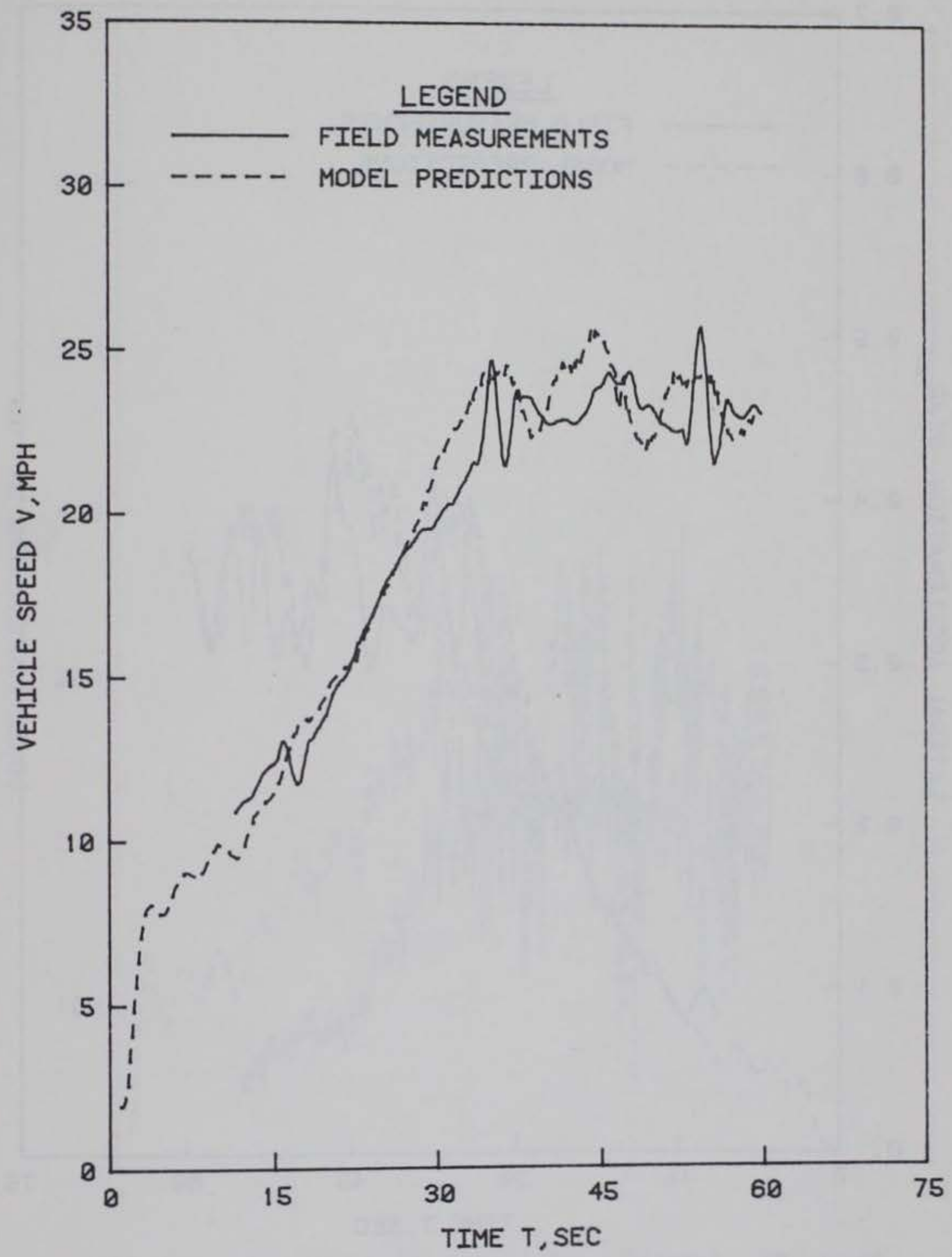

Figure 93. Vehicle speed-time history for test 140; comparison of model predictions with experimental data 


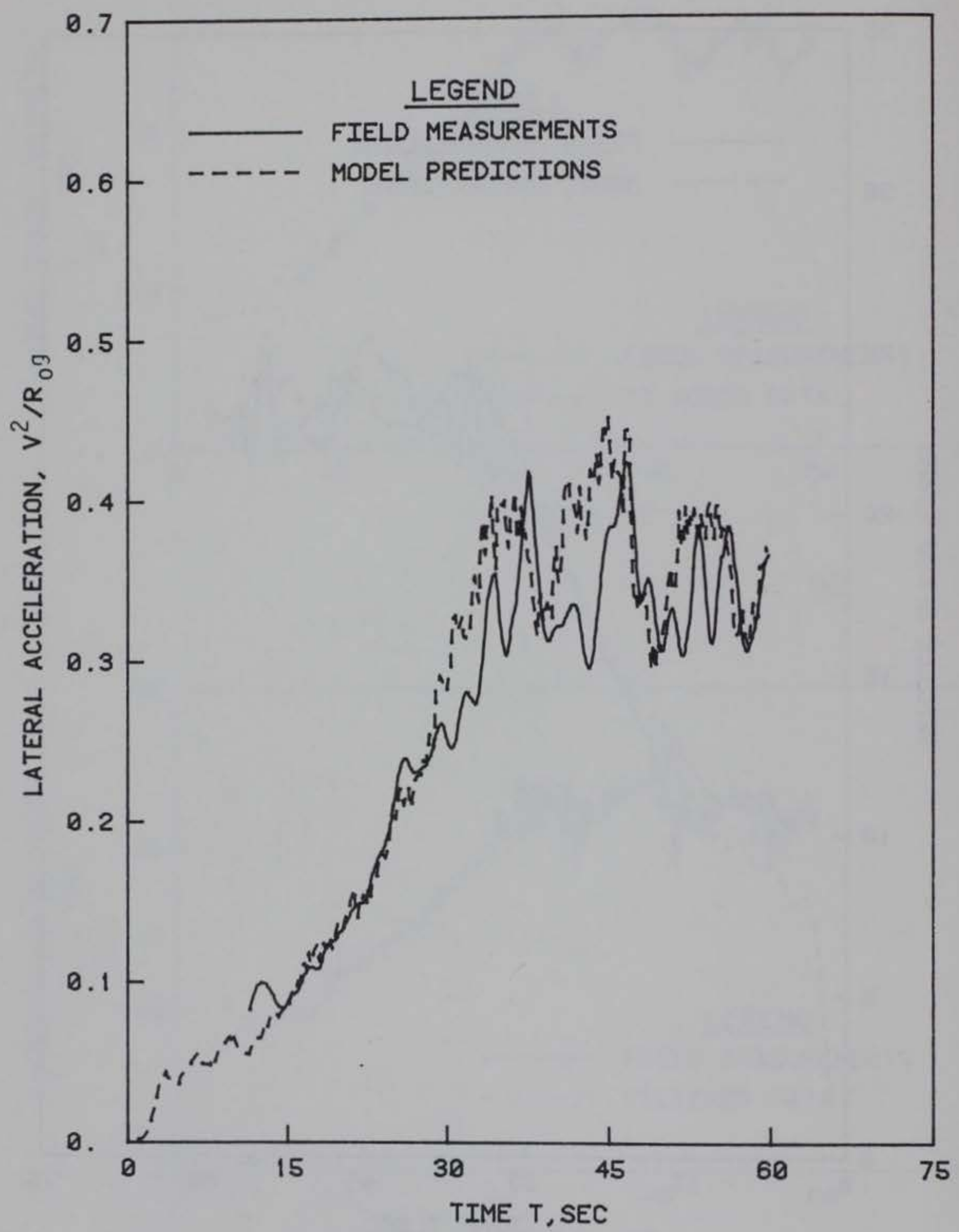

Figure 94. Lateral acceleration-time history for test 140; comparison of model predictions with experimental data 


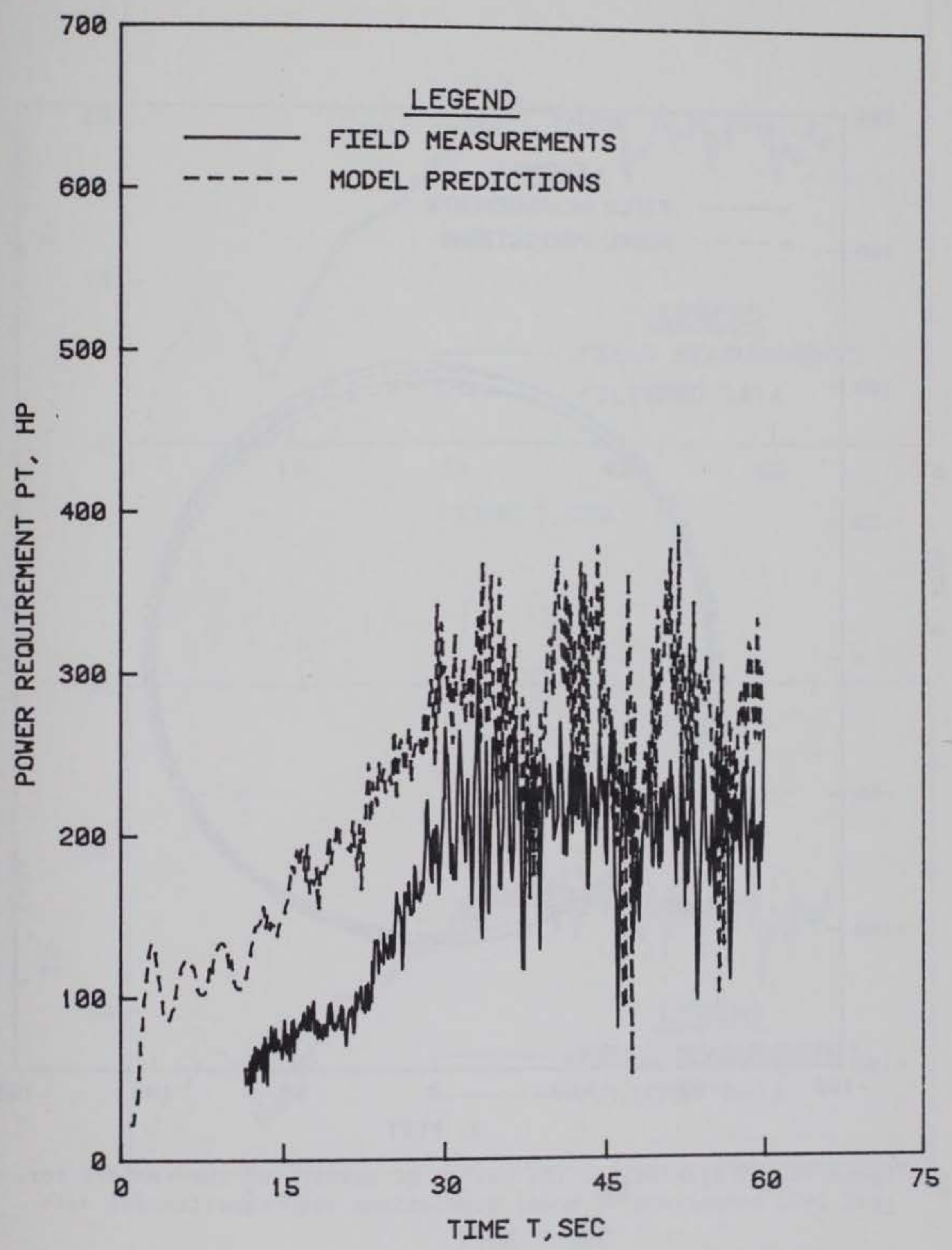

Figure 95. Total power-time history for test 140; comparison of model predictions with experimental data 


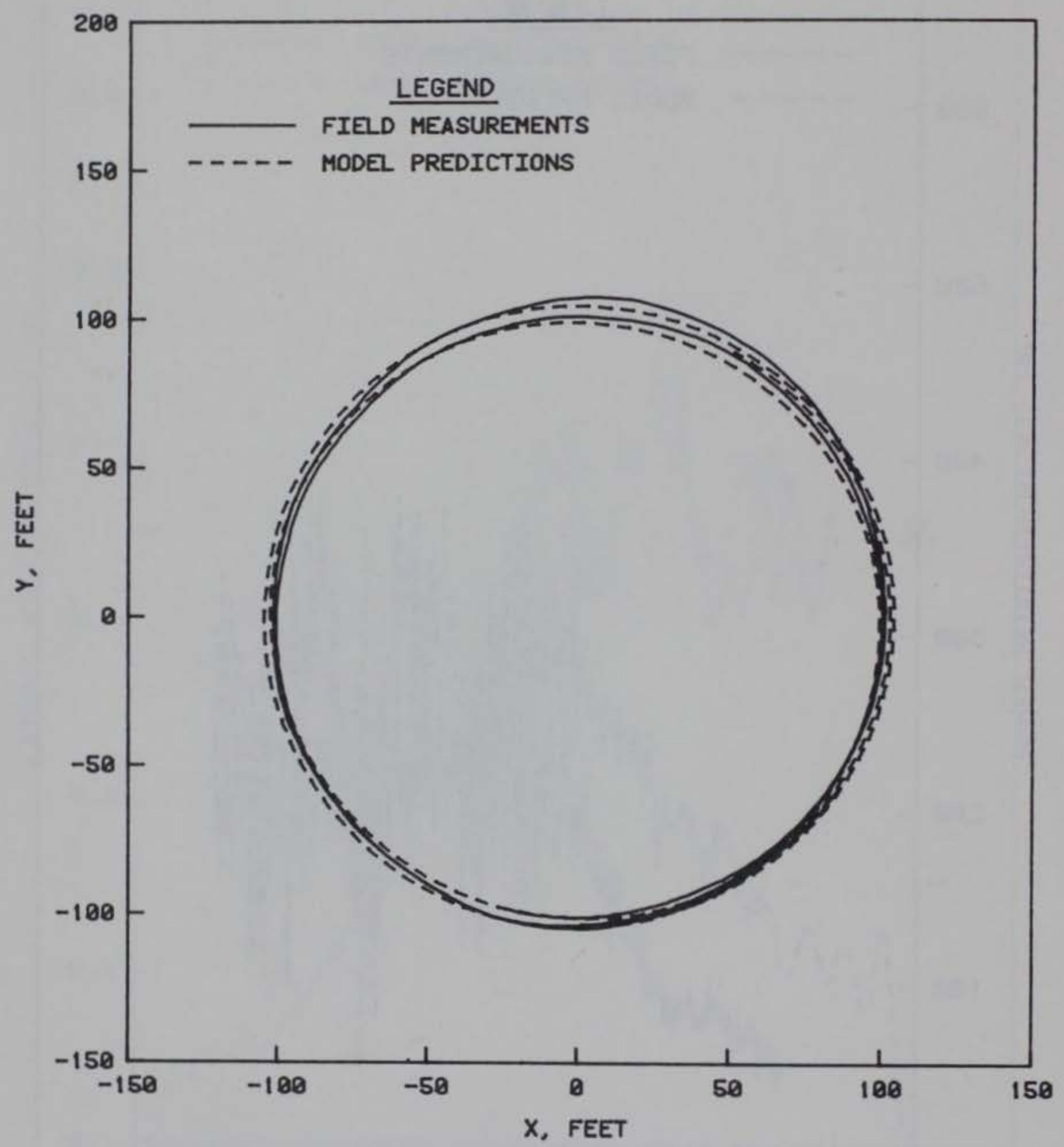

Figure 96. Trajectory of the center of gravity of the vehicle for test 140; comparison of model predictions with experimental data 

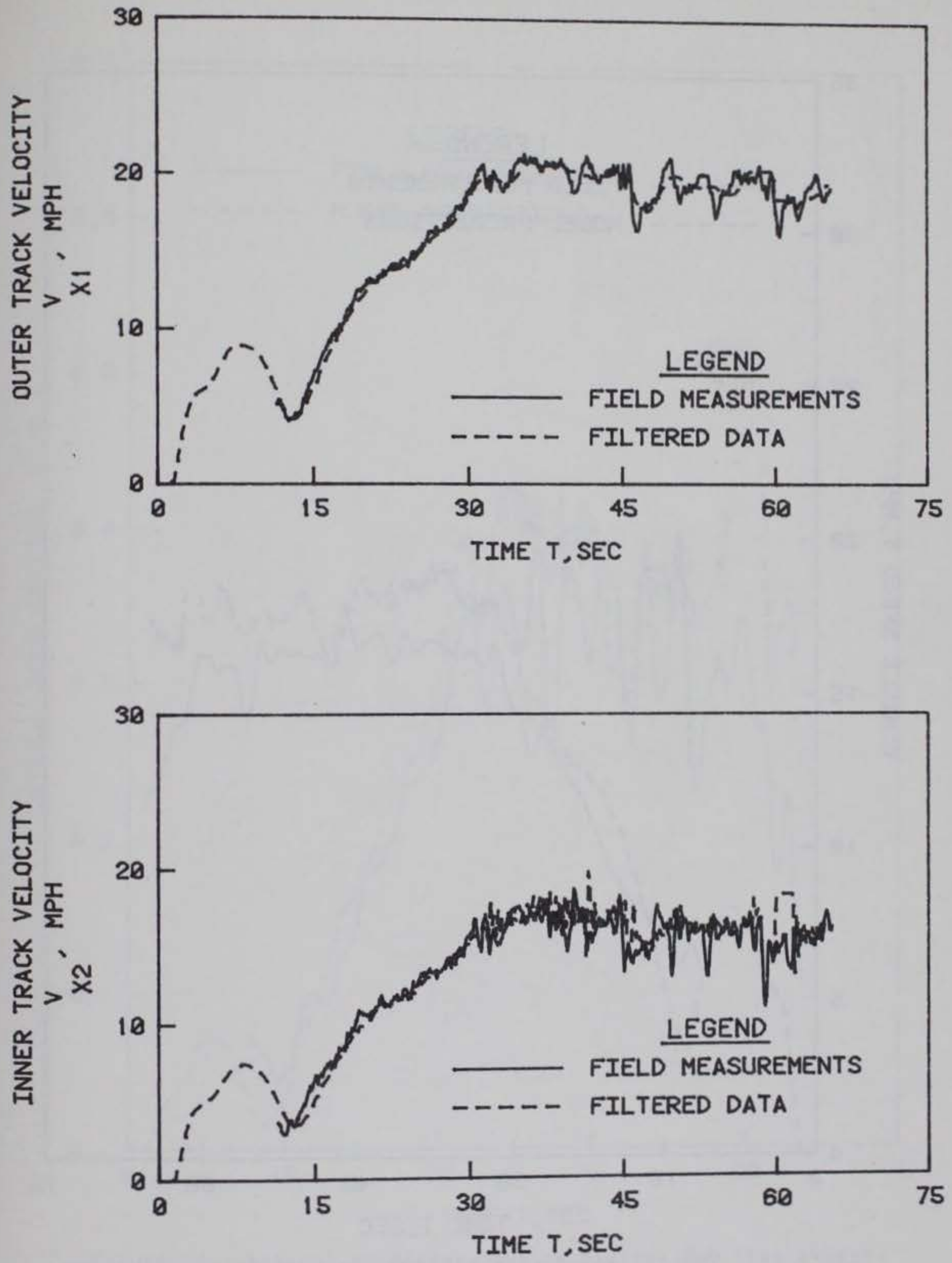

Figure 97. Outer and inner track velocity-time histories for test 141; field measurement and filtered data (input) 


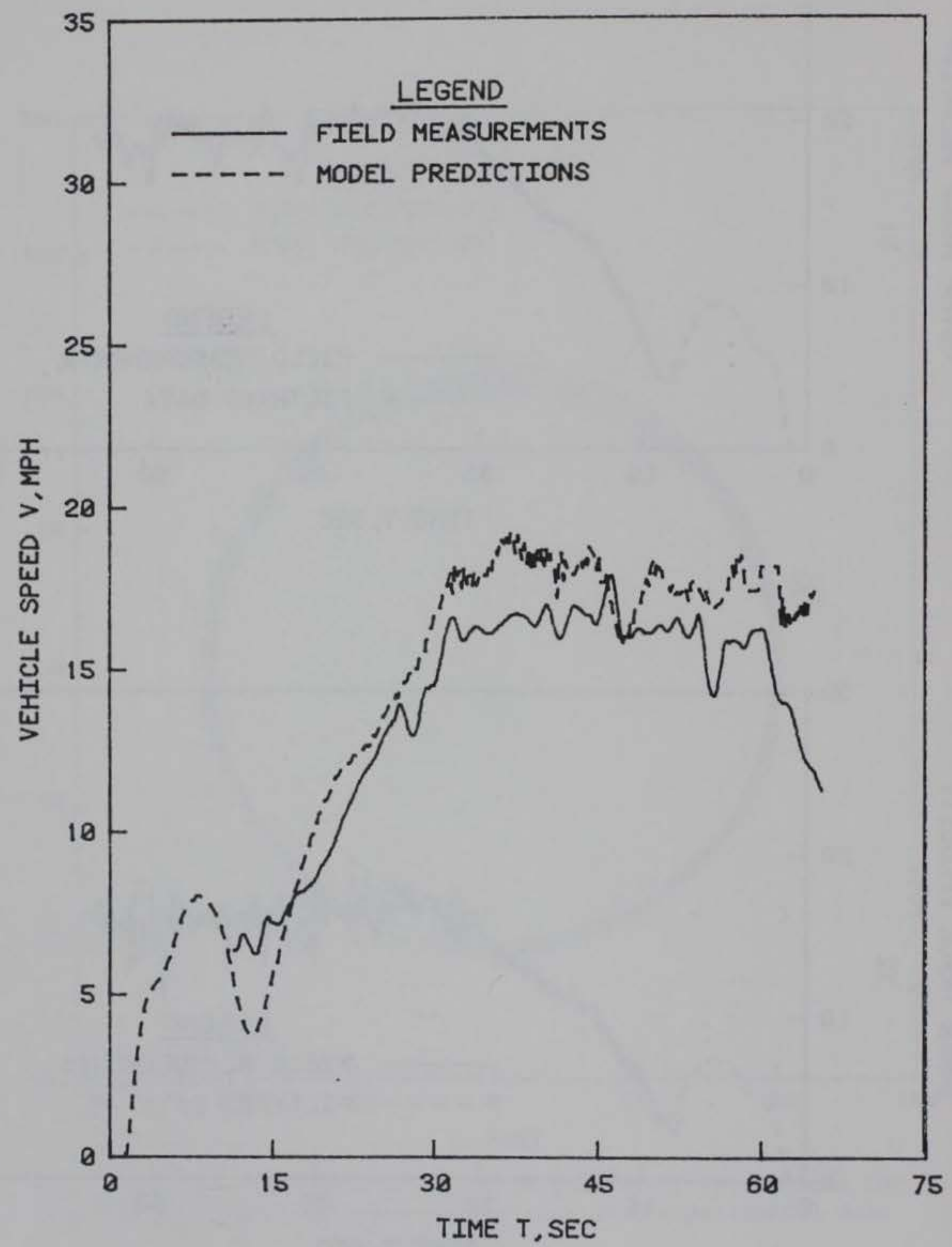

Figure 98. Vehicle speed-time history for test 141; comparison of model predictions with experimental data 


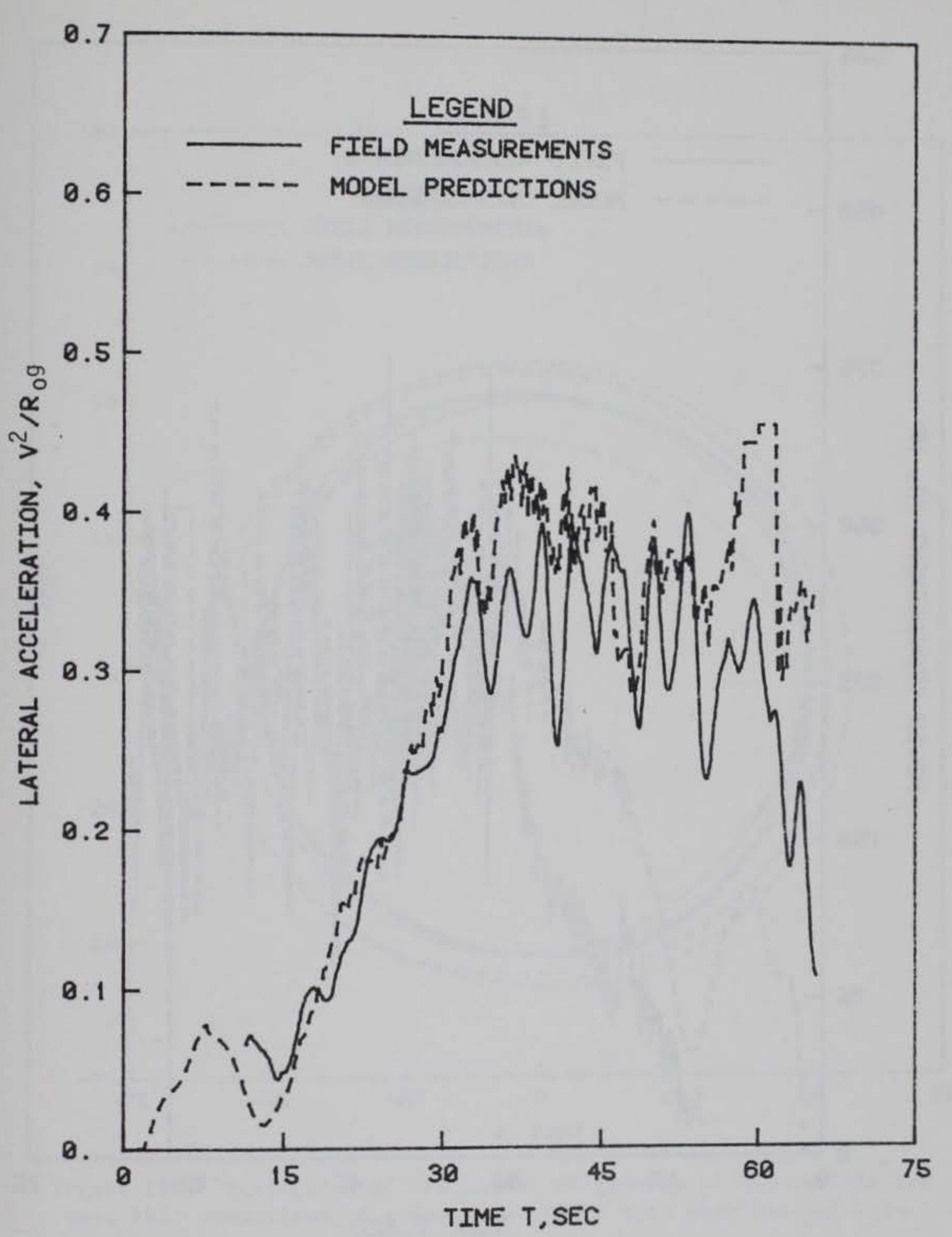

Figure 99. Lateral acceleration-time history for test 141; comparison of model predictions with experimental data

125 


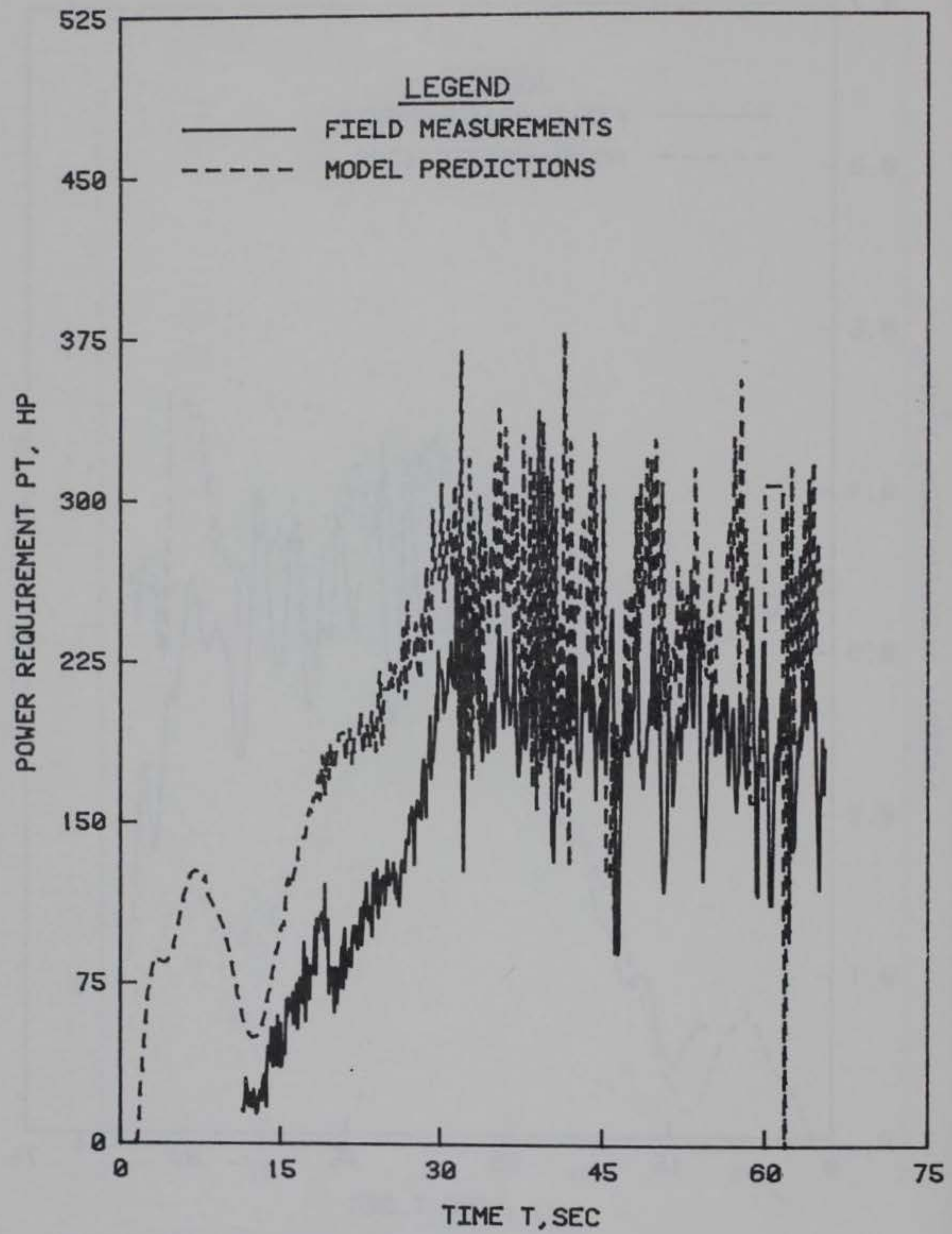

Figure 100. Total power-time history for test 141; comparison of model predictions with experimental data 


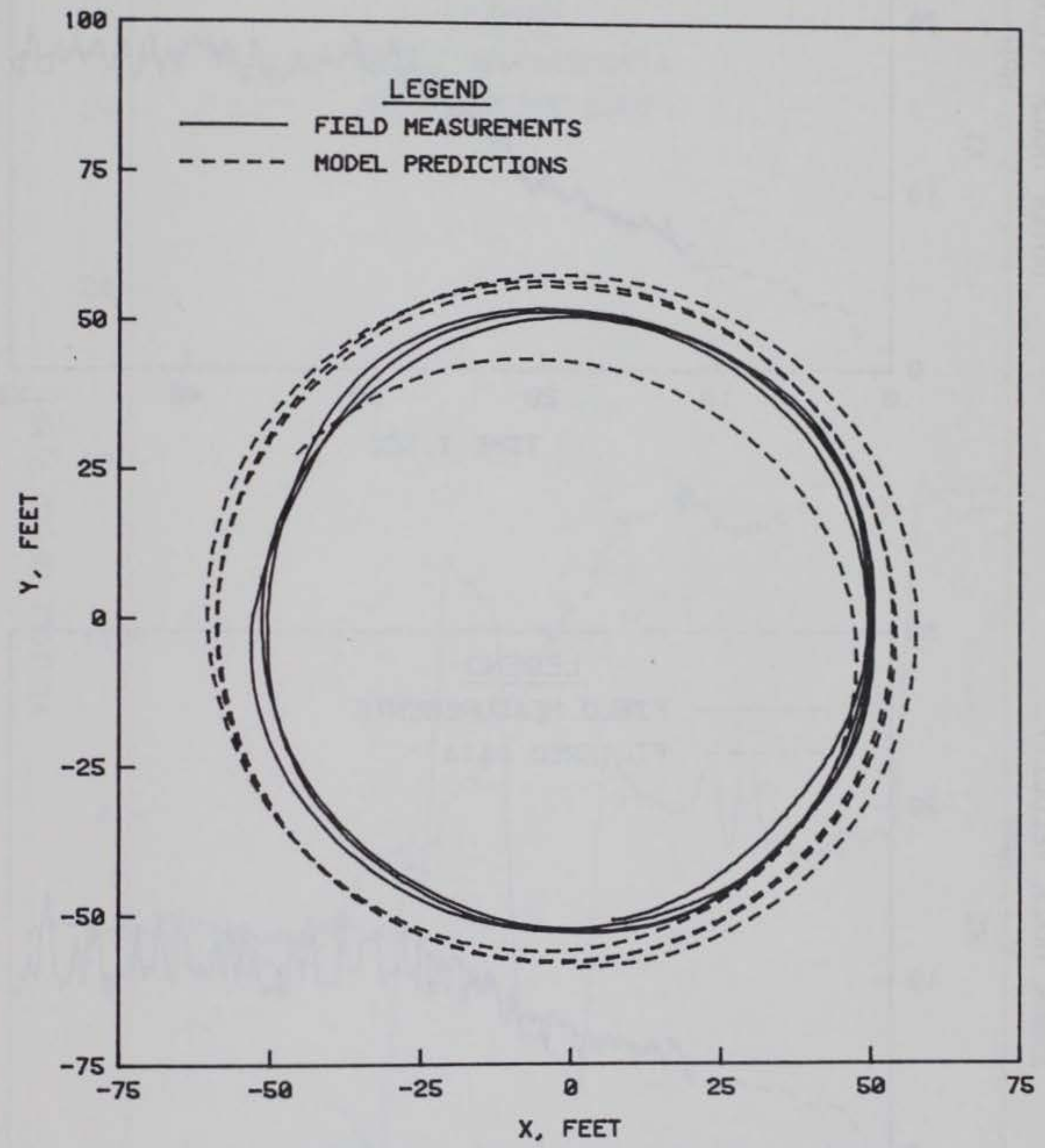

Figure 101. Trajectory of the center of gravity of the vehicle for test 141; comparison of model predictions with experimental data 

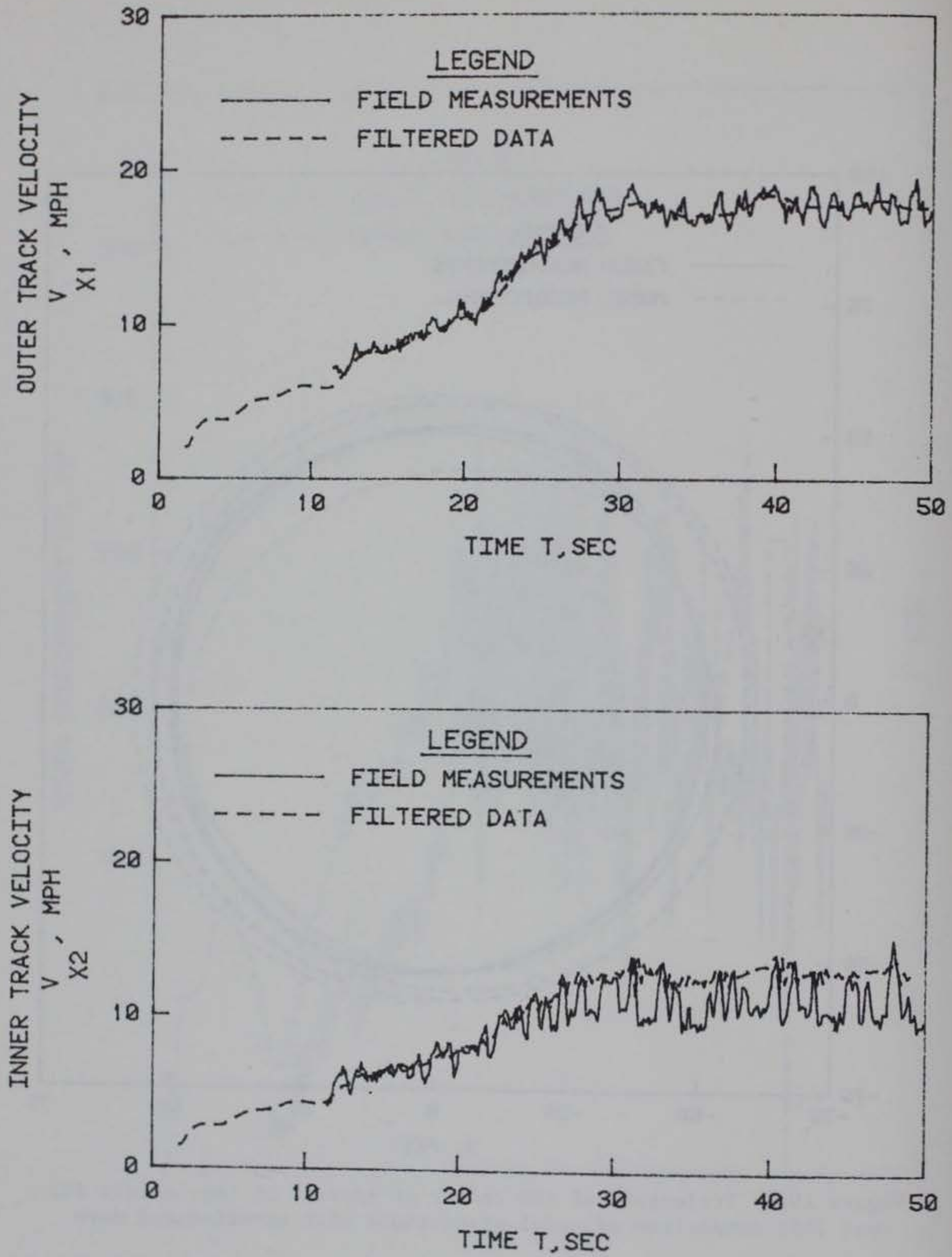

Figure 102. Outer and inner track velocity-time histories for test 157; field measurement and filtered data (input) 


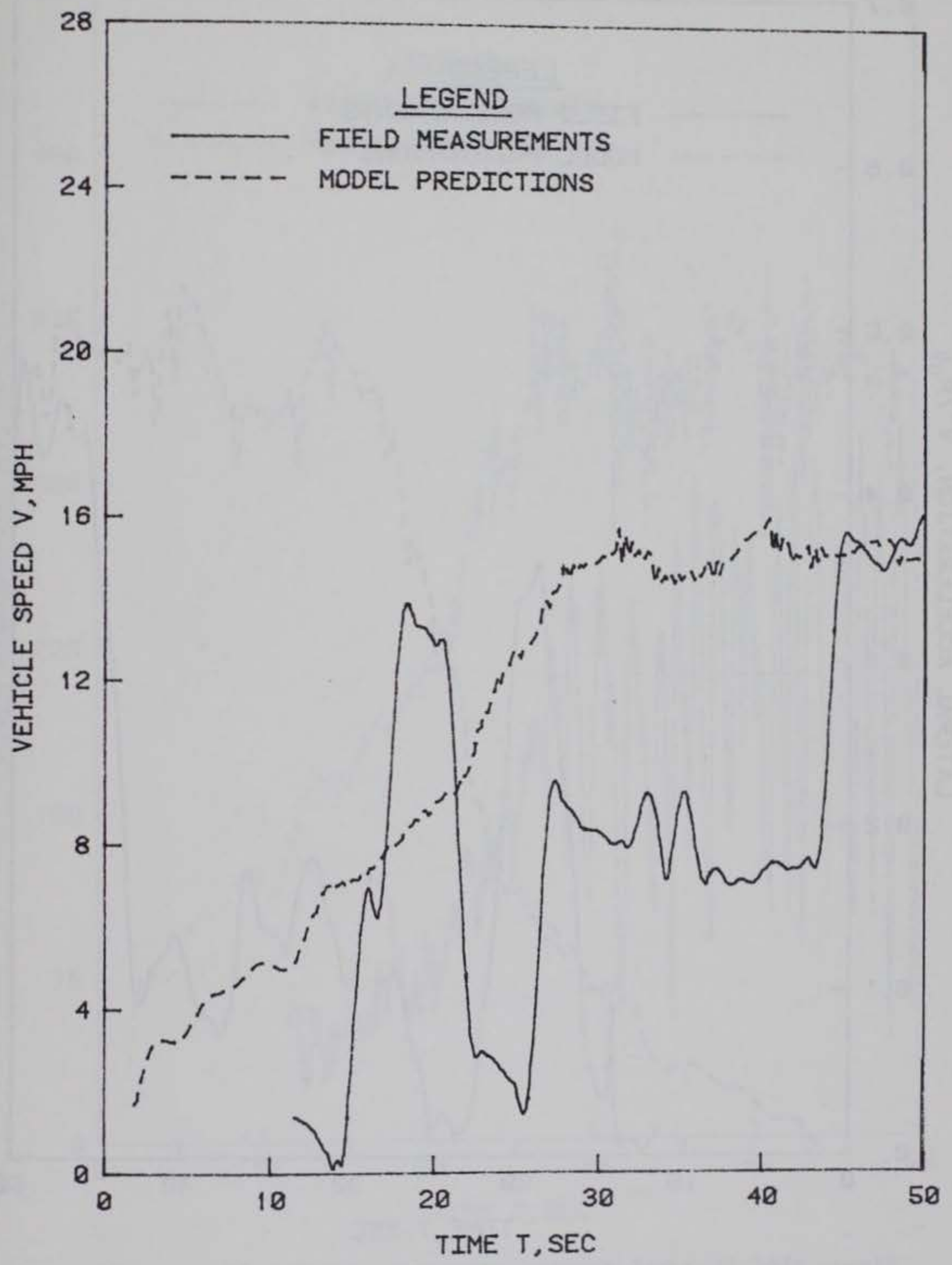

Figure 103. Vehicle speed-time history for test 157; comparison of model predictions with experimental data (example of poor quality data) 


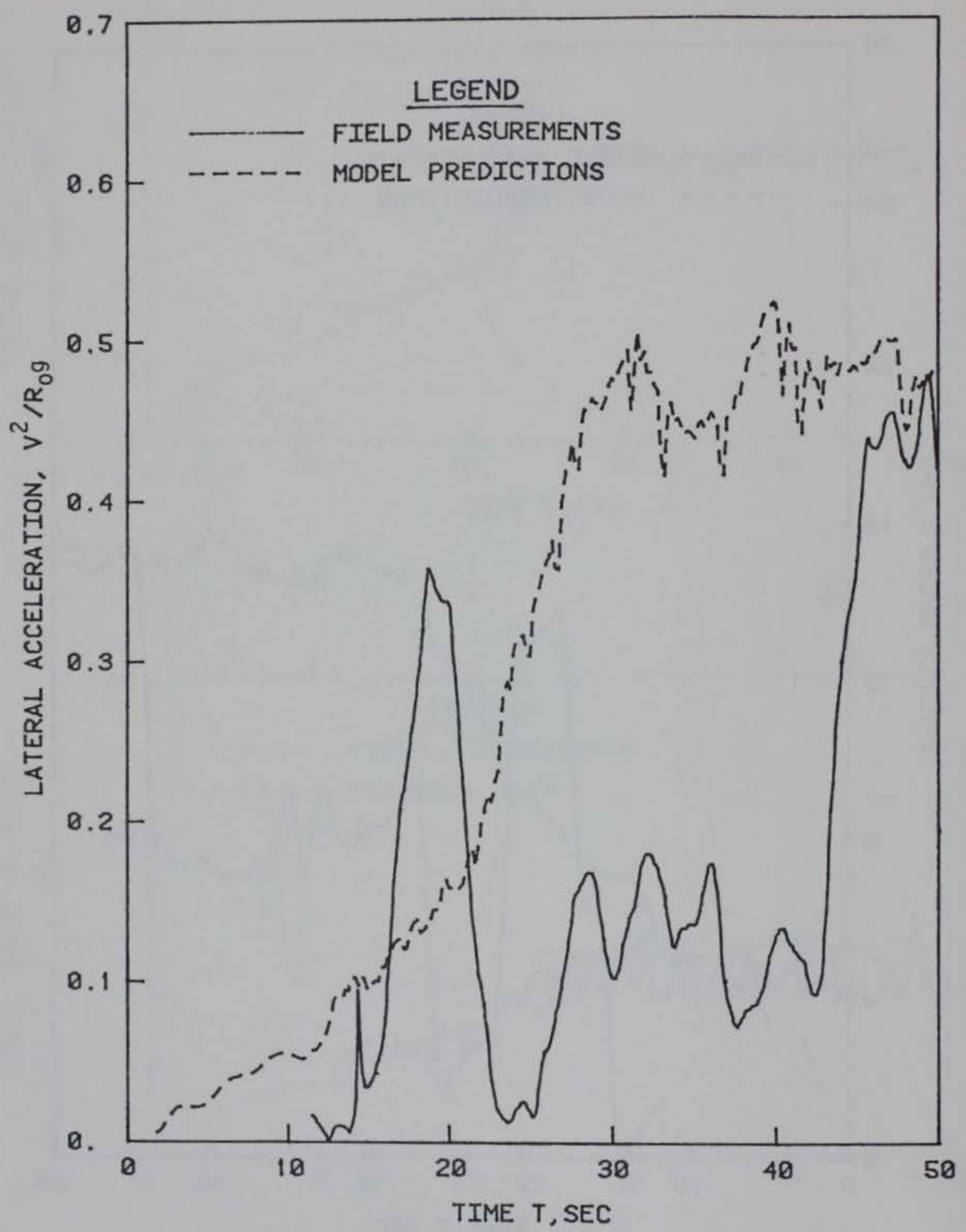

Figure 104. Lateral acceleration-time history for test 157; comparison of model predictions with experimental data (example of poor quality data) 


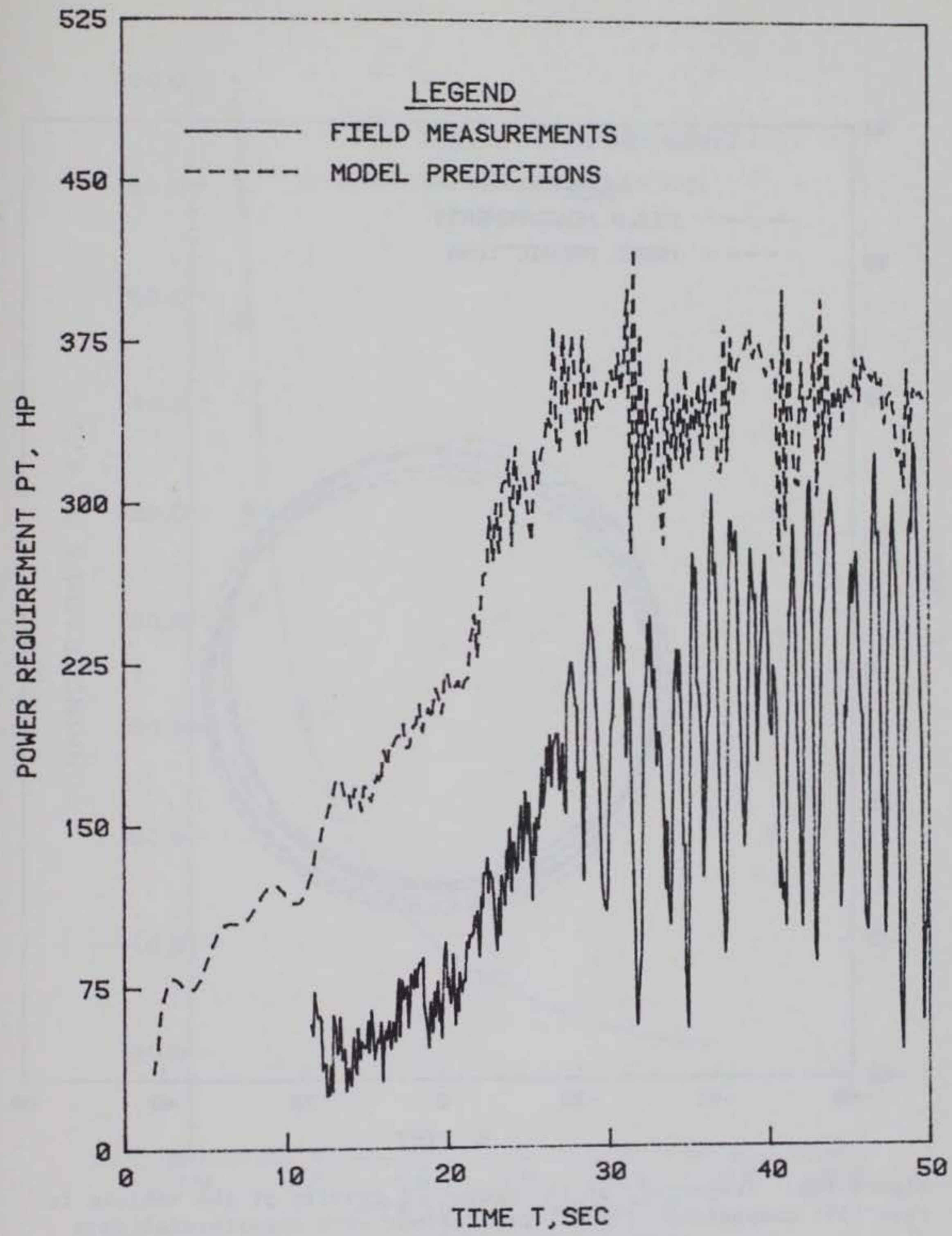

Figure 105. Total power-time history for test 157; comparison of model predictions with experimental data (example of poor quality data) 


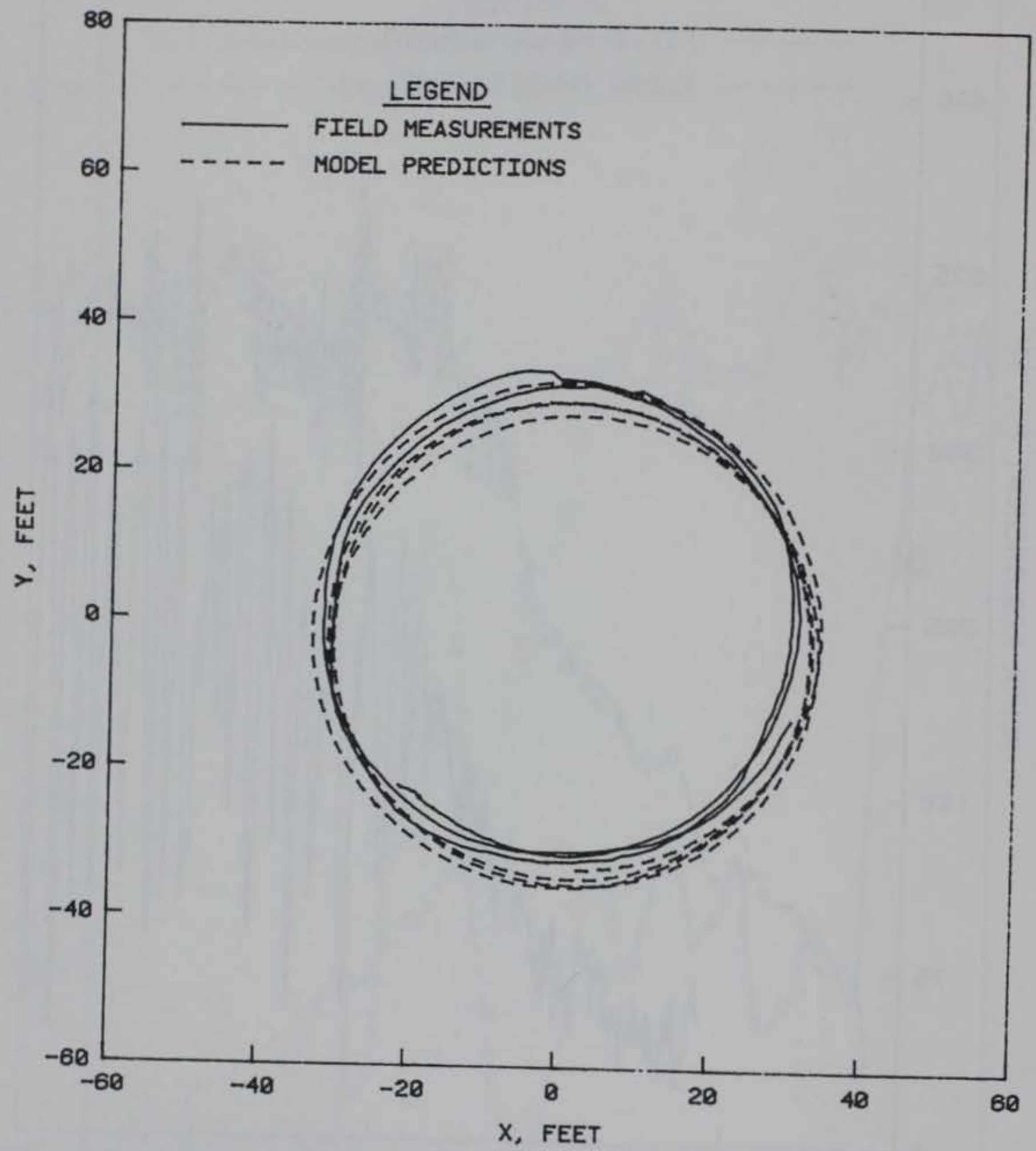
Figure 106. Trajectory of the center of gravity of the vehicle for
test 157; comparison of model predictions with experimental data (example of poor quality data) 


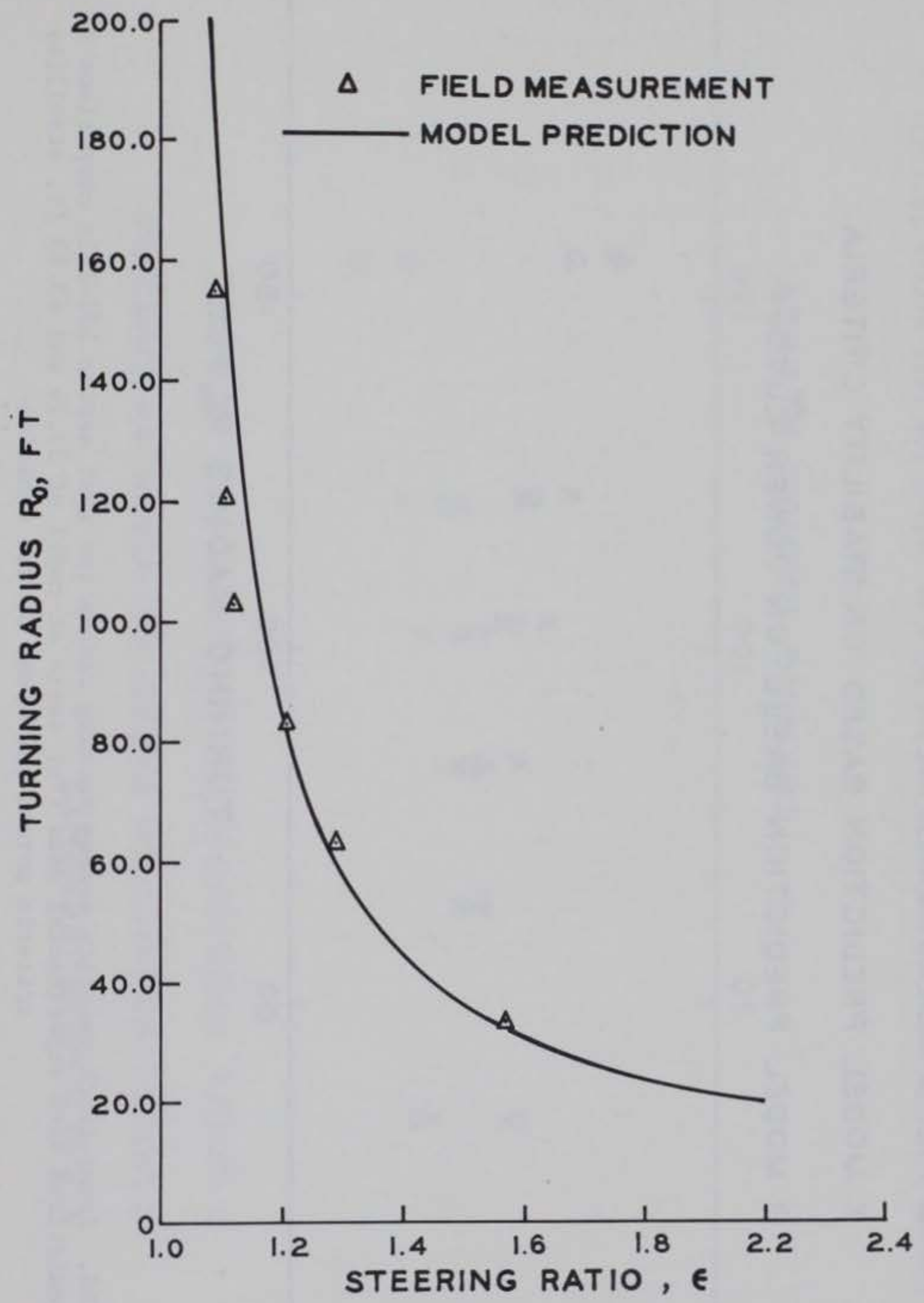

Figure 107. Turning radius versus steering ratio for test series 107-111; comparison of model predictions with experimental data 


\section{$\triangle$ FIELD MEASUREMENTS}

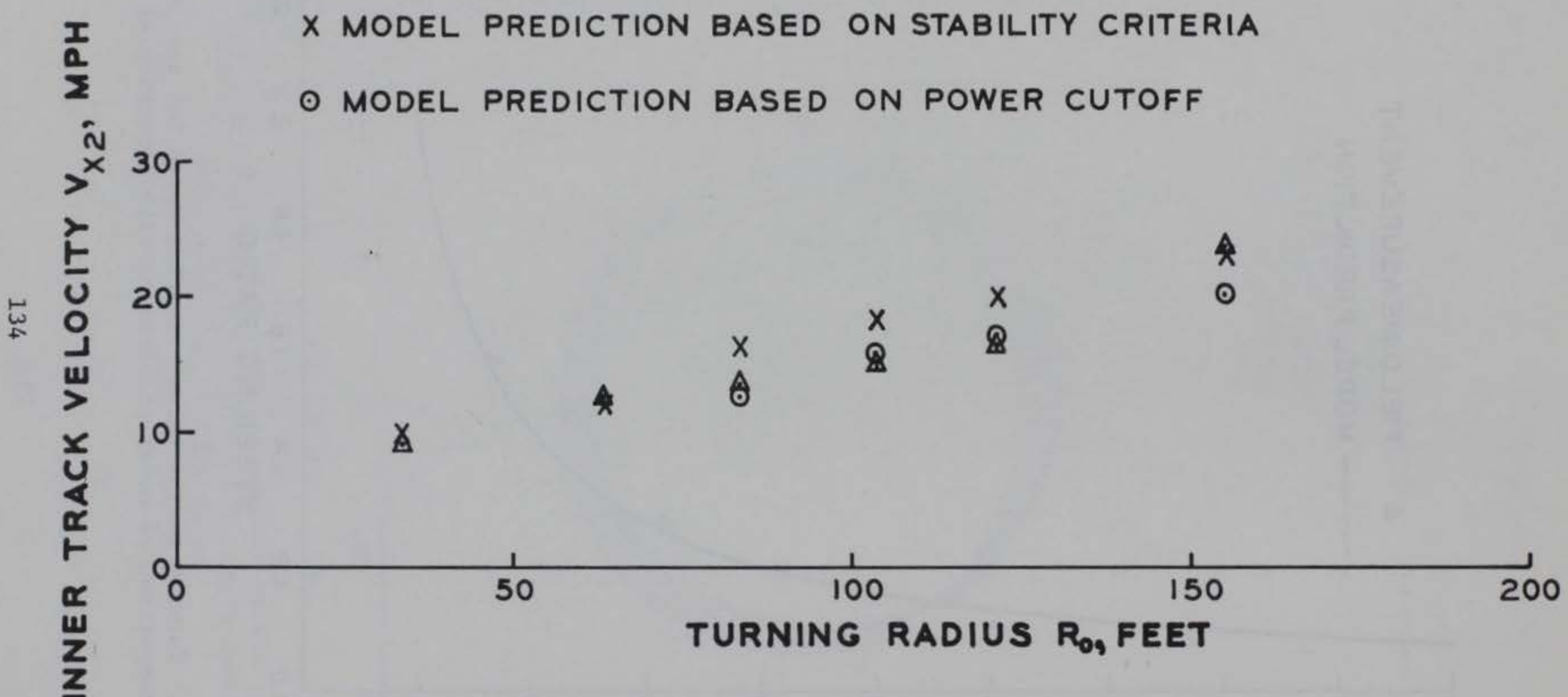

Figure 108. Inner track velocity versus turning radius for test series 107-111; comparison of model predictions with experimental data (for tests at radii of 33.74 and $63.69 \mathrm{ft}$, stability criteria govern the speed of the vehicle) 


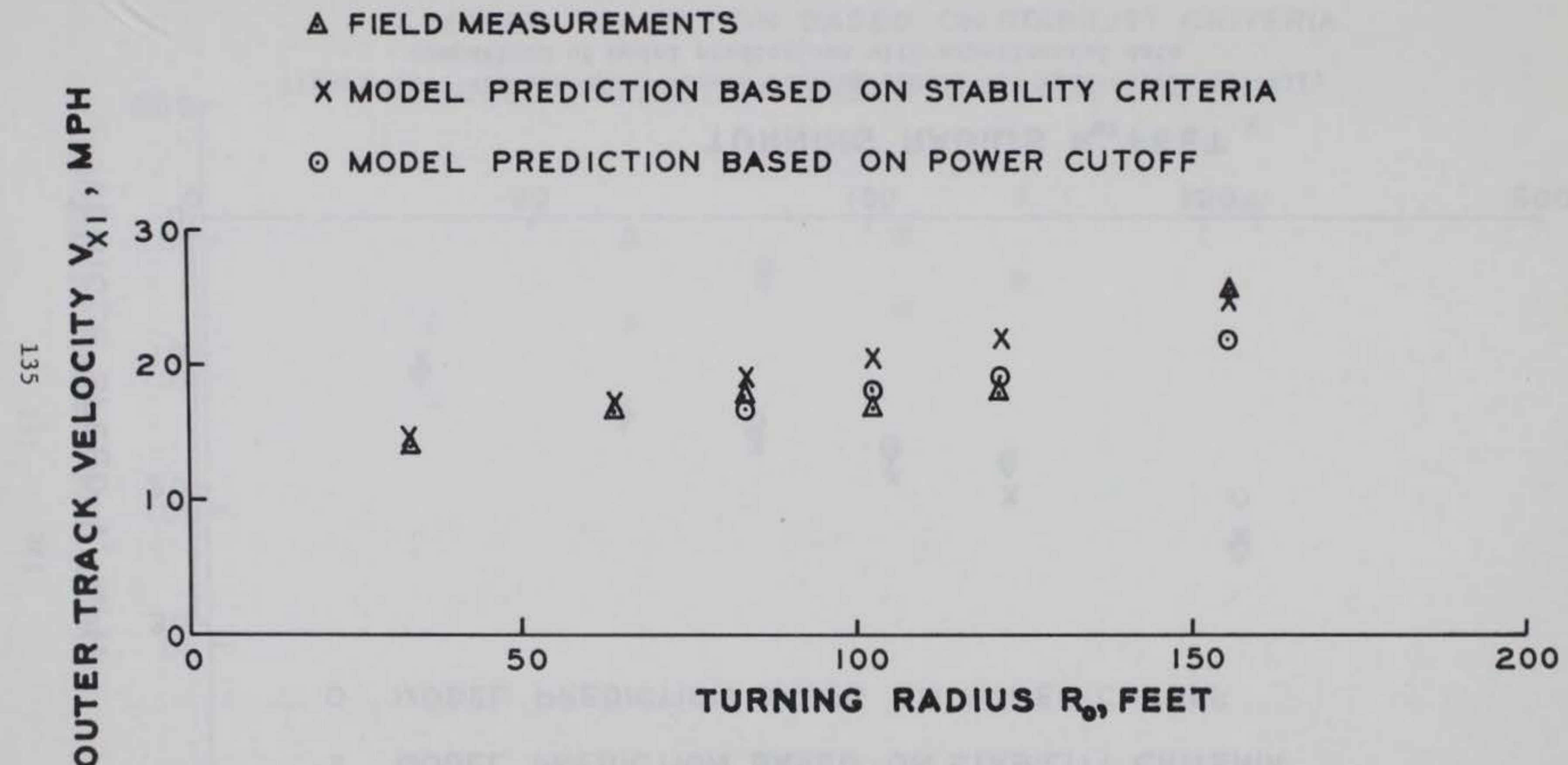

Figure 109. Outer track velocity versus turning radius for test series 107-111; comparison of model predictions with experimental data 
$\triangle$ FIELD MEASUREMENTS

$X$ MODEL PREDICTION BASED ON STABILITY CRITERIA

- MODEL PREDICTION BASED ON POWER CUTOFF

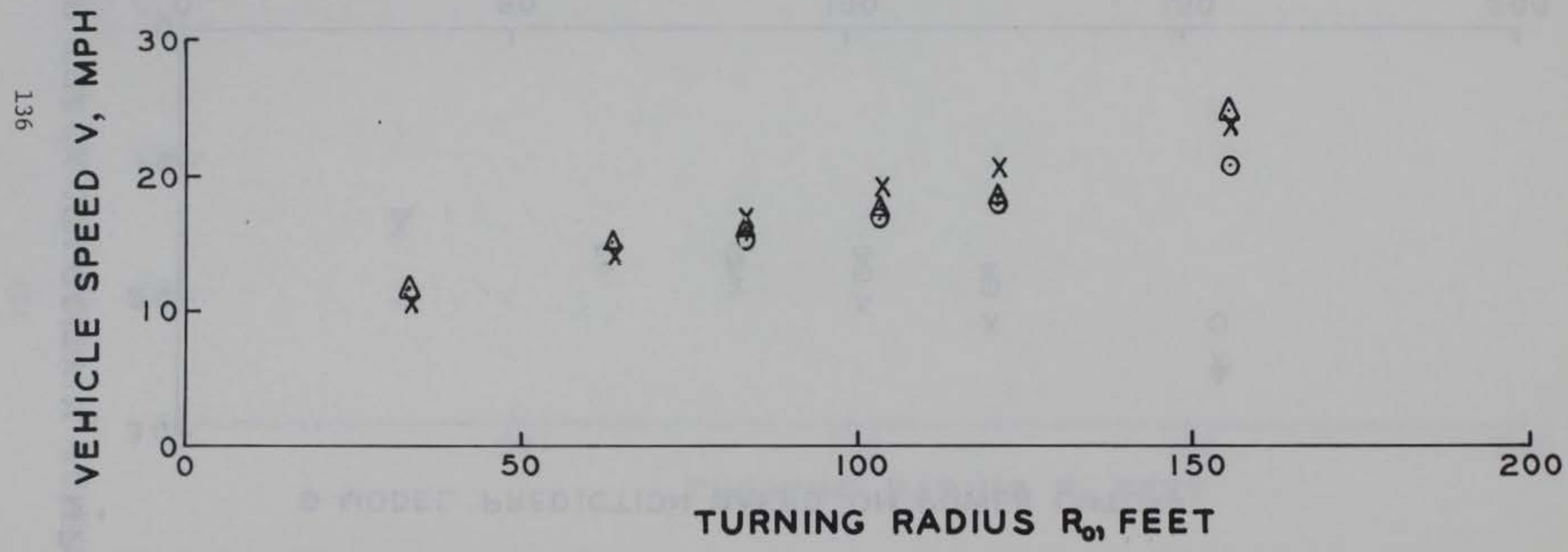

Figure 110. Vehicle speed versus turning radius for test series 107-111;

comparison of model predictions with experimental data 
$\triangle$ FIELD MEASUREMENTS

$X$ MODEL PREDICTION BASED ON STABILITY CRITERIA

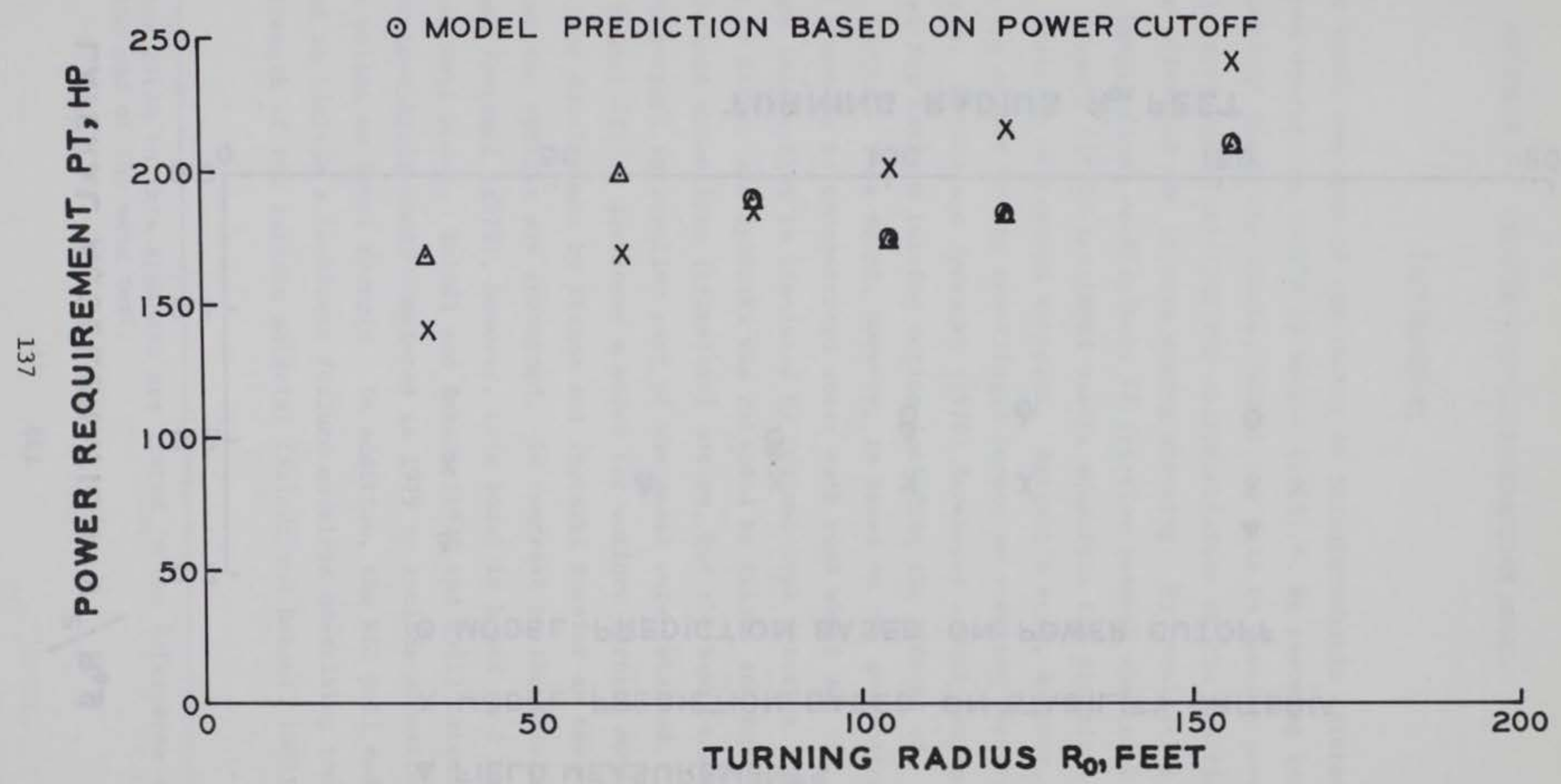

Figure 111. Power requirement versus turning radius for test series 107-111; comparison of model predictions with experimental data 


\section{$\triangle$ FIELD MEASUREMENTS}

늘

$X$ MODEL PREDICTION BASED ON STABILITY CRITERIA

○ MODEL PREDICTION BASED ON POWER CUTOFF

$\Delta$

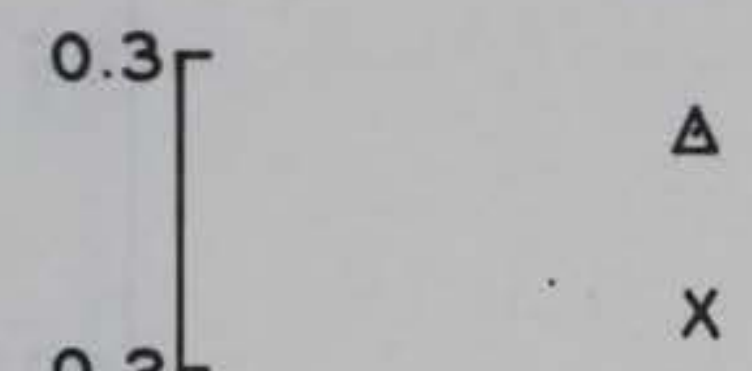

$\Delta$

$x$

* $\quad$ ธิ

$\begin{array}{lll}x & x & x \\ 8 & 0 & 0\end{array}$

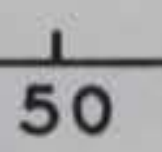

100

$\frac{1}{150}$

200

TURNING RADIUS Ro, FEET

Figure 112. Lateral acceleration versus turning radius for test series 107-111;

comparison of model predictions with experimental data 
APPENDIX A: TERRAIN-VEHICLE INTERACTION MODEL

\section{Introduction}

1. The basic concepts of the theory of terrain-vehicle interaction were developed during the 1950's by Bekker (1963).* By assuming various load distributions along the tracks, Bekker was able to develop several mathematical expressions relating the characteristics of the vehicle and the tractive effort of the terrain during steering. By considering the lateral and longitudinal coefficients of friction between the track and the ground, Hayashi (1975) developed simple equations for practical analysis of steering of tracked vehicles. Hayashi's work, however, did not include the effect of the centrifugal forces on steering performance of the vehicle. Kitano and Jyorzaki (1976) developed a more comprehensive model for uniform turning motion including the effects of centrifugal forces. This model, however, is based on the assumption that ground pressure is concentrated under each road wheel and the terrain-track interaction is simulated by Coulomb-type friction. The model given by Kitano and Jyorzaki was extended by Kitano and Kuma (1977) to include nonuniform (transient) motion, but the basic elements of the terrain-track interaction part of the model were retained. Baladi and Rohani (1978) developed a model for uniform turning motion parallel to the development by Kitano and Jyorzaki insofar as the kinematics of the vehicle are concerned. In contrast to the development by Kitano and Jyorzaki (1976), however, this model is based on a more comprehensive soil model. Baladi and Rohani (1979 and 1981) extended the WES terrain-vehicle model completed in 1979 to include nonuniform (transient) motion on level terrain. In addition, the WES soil model was modified to include a nonlinear failure envelope describing the shearing strength of the terrain material (Baladi and Rohani, 1981).

* References cited in this appendix are listed in the References section at the end of the main text. 
In this appendix, the WES terrain-vehicle model is extended to include the treatment of nonuniform (transient) turning motion on sloping terrain.

\section{$\underline{\text { Soil Model }}$}

\section{Strength components}

2. One of the most important properties of soil affecting trafficability is in situ shear strength. It has been found experimentally that the shear strength of purely cohesive soils is relatively independent of the confining stress, but strongly affected by the time rate of shearing. On the other hand, the shear strength of purely frictional soils is found to be relatively independent of the time rate of loading, but strongly dependent on confining pressure. The shearing resistance of most soils, however, is due to both the frictional and cohesive components. The cohesive and frictional components of strength are usually added together in order to obtain the total shear strength of the material. For static loading (very slow rate of deformation), the shear failure envelope is defined by

$$
\tau_{M}=A-M \exp (-N \sigma)
$$

where

$\tau_{M}=$ the maximum shearing strength of the material

$\sigma=$ the normal stress

$A=$ the strength of the material when $\sigma$ is large

$\mathrm{A}-\mathrm{M}=\mathrm{C}=$ the strength of the material or cohesion when $\sigma=0$

$\mathrm{N}=\mathrm{a}$ material constant

Equation Al is shown graphically in Figure Al.

3. As noted previously, the shear strength of cohesive soils increases with the increasing rate of loading. For the range of loading rates associated with the motion of tracked vehicles, the contribution to cohesive strength due to dynamic loading can be expressed as 
$C_{d}[1-\exp (-\Lambda \dot{\Delta})]$, where $C_{d}$ and $\Lambda$ are material constants and $\dot{\Delta}$ is the time rate of shearing deformation. In view of the above expression, the dynamic failure criterion takes the following form:

$$
\tau_{M}=A+C_{d}[1-\exp (-\Lambda \dot{\Delta})]-M \exp (-N \sigma)
$$

When $\Lambda$ equals zero, the dynamic failure criterion (Equation A2) reduces to the static failure criterion (Equation Al). Both are shown graphically in Figure $\mathrm{Al}$.

Shear stress-shear deformation relation

4. Prior to failure, the shear stress-shear deformation characteristics of a variety of soils can be expressed by the following mathematical expression (Kondner, 1963):

$$
\tau=\frac{G \tau_{M} \Delta}{\tau_{M}+G|\Delta|}
$$

The behavior of Equation $\mathrm{A} 3$ is shown graphically in Figure A2, in which $\tau$ denotes shearing stress, $\Delta$ is shearing deformation, and $G$ is the initial shear stiffness coefficient. Substituting $\tau_{M}$ from Equation A2, the shear stress-shear deformation relation for soil becomes

$$
\tau=\frac{G\left[A+C_{d}-C_{d} \exp (-\Lambda \dot{\Delta})-M \exp (-N \sigma)\right] \Delta}{G|\Delta|+A+C_{d}-C_{d} \exp (-\Lambda \dot{\Delta})-M \exp (-N \sigma)}
$$

For purely cohesive soils, $N$ equals zero and $\tau$ is only a function of $\Delta$ and $\dot{\Delta}$. For cohesionless or granular soils, $M$ equals $A$, $\mathrm{C}_{\mathrm{d}}$ is zero, and $\tau$ is a function of $\Delta$ and $\sigma$. For mixed soils exhibiting shearing resistance due to both frictional and cohesive components, $\tau$ is dependent on $\Delta, \dot{\Delta}$, and $\sigma$. The qualitative behavior of Equation A4 for these three conditions is shown in Figure A3. It should be pointed out that Equation A4 reduces to the rigid 
plastic soil model often used in mobility studies when an extremely large value is specified for $G$ and $\Lambda$ and is set to zero.

5. An appropriate test for determining the numerical values of the six material constants in Equation A4 is an in situ direct shear test. A field direct shear device has been developed at the WES for this purpose. A description of this device and the method of analysis of the data obtained from the direct shear test are documented in Appendix C of a report by Baladi and Rohani in 1979. For completeness, this Appendix $\mathrm{C}$ is included herein as Appendix B.

\section{Derivation of Terrain-Vehicle Model}

Boundary condition

6. The geometry of the vehicle and the boundary conditions of the proposed model are shown schematically in Figure A4. The XYZ coordinates are the local coordinate system of which $\mathrm{X}$ is always the longitudinal axis of the vehicle and $Y$ is a transverse axis parallel to the ground. These axes intersect at the center of geometry of the vehicle 0 . The $Z$ axis is a vertical axis passing through the origin 0 . The center of gravity of the vehicle (CG) lies on the $X$ axis and is displaced by a distance $C_{X}$ from the origin. The numerical value of $C_{X}$ is assumed to be positive if $C G$ is displaced forward from the center of geometry of the vehicle. The XY coordinates of the instantaneous center of rotation (ICR) are $P+C_{X}$ and $\tilde{R}$, respectively, where $P$ is the offset. The center of rotation and the radius of the trajectory of the CG are, respectively, CR and $\mathrm{R}_{\mathrm{o}}$. The height of the center of gravity measured from ground surface is denoted by $\mathrm{H}$. The lengths of the track-ground contact, the track width, and the tread of the tracks are L, D, and B, respectively. As shown in Figure $\mathrm{A} 4$, the components of the inertial forces $\mathrm{F}_{\mathrm{C}}$ in $\mathrm{X}$ and $\mathrm{Y}$ directions are, respectively, $\mathrm{F}_{\mathrm{CX}}$ and $\mathrm{F}_{\mathrm{CY}}$. The weight of the vehicle is $W$.

Stress distribution along the tracks

7. Two types of stress (i.e., normal and shear stresses) exist 
along the track. As indicated in Figure A4, the normal stresses under the outer and inner tracks are denoted by $R_{1}(X)$ and $R_{2}(X)$, respectively. The components of the shear stress in $X$ and $Y$ directions are, respectively, $T_{1}(x)$ and $Q_{1}(x)$ for the outer track, and $T_{2}(x)$ and $\mathrm{Q}_{2}(\mathrm{x})$ for the inner track. These stresses are dependent on the terrain type, vehicle configuration, and speed and turning radius of the vehicle.

8. The magnitude of normal stresses $R_{1}(x)$ and $R_{2}(x)$ can be determined in terms of the components of the inertial force, the track tensions, and the characteristics of the vehicle by considering the balance of vertical stresses and their moments in Figure A4. Thus

$$
\begin{aligned}
& R_{1}(x)=\frac{W}{d^{2}}\left[\frac{1}{2}+6 x c x-\frac{h}{b} \frac{F_{C Y}}{W}-6 h x \frac{F_{C X}}{W}+\frac{d^{2}{ }^{2} N_{1}(x)}{W}\right] \\
& R_{2}(x)=\frac{W}{d^{2}}\left[\frac{1}{2}+6 x c x+\frac{h}{b} \frac{F_{C Y}}{W}-6 h x \frac{F_{C Y}}{W}+\frac{d^{2}{ }^{2} N_{2}(x)}{W}\right]
\end{aligned}
$$

where

$$
\begin{aligned}
\mathrm{h} & =\mathrm{H} / \mathrm{L} \\
\mathrm{b} & =\mathrm{B} / \mathrm{L} \\
\mathrm{d} & =\mathrm{D} / \mathrm{L} \\
\mathrm{c}_{\mathrm{X}} & =\mathrm{C}_{\mathrm{X}} / \mathrm{L} \\
\mathrm{x} & =\mathrm{X} / \mathrm{L} \\
\mathrm{y} & =\mathrm{Y} / \mathrm{L} \\
\mathrm{z} & =\mathrm{Z} / \mathrm{L}
\end{aligned}
$$

$\mathrm{N}_{1}(\mathrm{x})$ and $\mathrm{N}_{2}(\mathrm{x})=$ contributions due to track tension.

9. The components of the shear stress in the $X$ and $Y$ directions along both the outer and inner tracks can be obtained by combining 
Equations $\mathrm{A} 4, \mathrm{~A} 5$, and $\mathrm{A6}$.* Thus (it is noted that $\mathrm{R}_{1}$ and $\mathrm{R}_{2}$ replace the normal stress $\sigma$ in Equation A4)

$$
\begin{aligned}
& T_{i}(x)=\frac{W}{L^{2}} \mu \delta_{i}\left\{\frac{d a+d c_{d}-d c_{d} \exp \left(-\lambda \dot{\delta}_{i}\right)-m \exp \left[-n r_{i}(x)\right]}{\mu\left|\delta_{i}\right| d+d a+d c_{d}-d c_{d} \exp \left(-\lambda \dot{\delta}_{i}\right)-m \exp \left[-n r_{i}(x)\right]}\right\} \cos \gamma_{i} \text { (A7) } \\
& Q_{i}(x)=\frac{W}{L^{2}} \mu \delta_{i}\left\{\frac{d a+d c_{d}-d c_{d} \exp \left(-\lambda \dot{\delta}_{i}\right)-m \exp \left[-n r_{i}(x)\right]}{\mu\left|\delta_{i}\right| d+d a+d c_{d}-d c_{d} \exp \left(-\lambda \dot{\delta}_{i}\right)-m \exp \left[-n r_{i}(x)\right]}\right\} \sin \gamma_{i} \text { (A8) }
\end{aligned}
$$

where

$$
\begin{aligned}
i & =1,2 \\
\mathrm{r}_{i}(\mathrm{x}) & =\mathrm{dL}^{2} \mathrm{R}_{\mathrm{i}}(\mathrm{x}) / \mathrm{W} \\
\delta_{i} & =\Delta_{i} / \mathrm{L} \\
\dot{\delta}_{i} & =\dot{\Delta}_{i} / \mathrm{L} \\
\mu & =\mathrm{GL}^{3} / \mathrm{W} \\
\lambda & =\Lambda \mathrm{L} \\
\mathrm{a} & =\mathrm{AL}^{2} / \mathrm{W} \\
\mathrm{m} & =\mathrm{ML}^{2} / \mathrm{W} \\
\mathrm{n} & =\mathrm{NW}^{2} / \mathrm{L}^{2} \\
\mathrm{c}_{\mathrm{d}} & =\mathrm{C}_{\mathrm{d}} \mathrm{L}^{2} / \mathrm{W}
\end{aligned}
$$

The variables $\gamma_{1}$ and $\gamma_{2}$, in Equations $A 7$ and A8, are the slip angles and can be written as

* To account for the effect of the size of the shear box on the shear stiffness $G$, the measured value of $G$ is normalized by multiplying it by $4 / \mathrm{L}$ (the length of the shear box $=4$ in.). 


$$
\left.\begin{array}{l}
\gamma_{1}=\tan ^{-1} \frac{X-P-C_{X}}{C_{1}}=\tan ^{-1} \frac{x-p-c_{X}}{\xi_{1}} \\
\gamma_{2}=\tan ^{-1} \frac{X-P-C_{X}}{C_{2}}=\tan ^{-1} \frac{x-p-c_{X}}{\xi_{2}}
\end{array}\right\}
$$

where

$$
\begin{aligned}
\xi_{1} & =C_{1} / L \\
\xi_{2} & =C_{2} / L \\
p & =P / L
\end{aligned}
$$

The parameter $C_{1}$ is the distance between the instantaneous center of rotation of the outer track $I_{1}$ and its axis of symmetry, and $C_{2}$ is the distance between the instantaneous center of rotation of the inner track $\mathrm{IC}_{2}$ and its axis of symmetry (Figure A5).

10. In order to use Equations A7 through A9, the normal stress contributions due to track tensions $\mathrm{N}_{1}(\mathrm{x})$ and $\mathrm{N}_{2}(\mathrm{x})$, the track slip velocities and displacements (i.e., $\dot{\Delta}_{1}, \Delta_{1}, \dot{\Delta}_{2}$, and $\Delta_{2}$ ), and the inertial forces $\mathrm{F}_{\mathrm{CX}}$ and $\mathrm{F}_{\mathrm{CY}}$ have to be determined. These factors are discussed in the following paragraphs.

\section{Normal stress contribution due to track tension}

11. The effect of track tension on the normal stress distribution is influenced considerably by the motion of the vehicle. At relatively low speed, tractive effort is applied to the outer track, while braking force is applied to the inner track (Figure A6a). At high speed, on the other hand, tractive efforts are applied to both tracks (Figure A6b).

12. The angles $\theta_{\mathrm{a}}$ and $\theta_{\mathrm{d}}$ in Figure $\mathrm{A6}$ are the approach and departure angles of the track envelope, respectively. The forces $\overline{\mathrm{T}}_{1}$ and $\bar{T}_{2}$ are the track tension in the outer and inner track, respective1y. These forces can be obtained by integrating Equation A7. Thus 


$$
\begin{aligned}
& \overline{\mathrm{T}}_{1}=\mathrm{L}_{-\frac{1}{2} \int_{1}^{\frac{1}{2}} \mathrm{~T}_{1}(\mathrm{x}) \mathrm{dx}} \\
& \overline{\mathrm{T}}_{2}=\mathrm{L}^{2} \int_{-\frac{1}{2}}^{\frac{1}{2}} \mathrm{~T}_{2}(\mathrm{x}) \mathrm{dx}
\end{aligned}
$$

The normal stress distributions are influenced, however, by the vertical components of the forces $\bar{T}_{1}$ and $\bar{T}_{2}$; namely, $n_{1}, n_{2}$, and $n_{2}^{\prime}$. The values of $n_{1}, n_{2}$, and $n_{2}^{\prime}$ are

$$
\begin{gathered}
\mathrm{n}_{1}=\overline{\mathrm{T}}_{1} \sin \theta_{\mathrm{d}} \\
\mathrm{n}_{2}=\left\{\begin{array}{lll}
\overline{\mathrm{T}}_{2} \sin \theta_{\mathrm{a}} & \text { if } & \xi_{2} \geq 0 \\
0 & \text { if } & \xi_{2}<0
\end{array}\right\} \\
\mathrm{n}_{2}^{\prime}=\left\{\begin{array}{lll}
-\overline{\mathrm{T}}_{2} \sin \theta_{\mathrm{d}} & \text { if } & \xi_{2}<0 \\
0 & \text { if } & \xi_{2} \geq 0
\end{array}\right\}
\end{gathered}
$$

With the determination of the forces $n_{1}, n_{2}$, and $n_{2}^{\prime}$, the normal stress contributions due to track tension may be determined.

13. Since the tracks are assumed to be rigid, the normal stresses due to track tension may be distributed according to the following equations (Figure A7): 


$$
\left.\begin{array}{ll}
\mathrm{N}_{1}(\mathrm{x})=\mathrm{ax}+\mathrm{m}_{0} & \text { for } \quad \frac{\ell}{\mathrm{L}}-\frac{1}{2} \leq \mathrm{x} \leq \frac{1}{2} \\
\mathrm{~N}_{1}(\mathrm{x})=\mathrm{ax}+\mathrm{m}_{0}+\frac{2 \mathrm{n}_{1}}{\mathrm{~d} \ell^{2}}\left(\mathrm{x}+\frac{1}{2}-\frac{\ell}{\mathrm{L}}\right) & \text { for }-\frac{1}{2} \leq \mathrm{x} \leq \frac{\ell}{\mathrm{L}}-\frac{1}{2}
\end{array}\right\}
$$

and

$$
\left.\begin{array}{ll}
\mathrm{N}_{2}(x)=a x+m_{I}-\frac{2 n_{2}}{d \ell^{2}}\left(x-\frac{1}{2}+\frac{\ell}{L}\right) & \text { for } \quad \frac{1}{2}-\frac{\ell}{L} \leq x \leq \frac{1}{2} \\
N_{2}(x)=a x+m_{I} & \text { for } \quad \frac{\ell}{L}-\frac{1}{2} \leq x \leq \frac{1}{2}-\frac{\ell}{L} \\
N_{2}(x)=a x+m_{I}+\frac{2 n_{2}^{\prime}}{d \ell^{2}}\left(x+\frac{1}{2}-\frac{\ell}{L}\right) & \text { for }-\frac{1}{2} \leq x \leq \frac{\ell}{L}-\frac{1}{2}
\end{array}\right\}
$$

in which $\ell$ is the distance between two adjacent wheels, and a , $\mathrm{m}_{0}$, and $\mathrm{m}_{\mathrm{I}}$ can be determined by considering the equation of equilibrium of normal stresses and the moments of these stresses. Thus

$$
\begin{aligned}
& \int_{-\frac{1}{2}}^{\frac{1}{2}}\left(a x+m_{0}\right) d x+\int_{-\frac{1}{2}}^{\frac{\ell}{L}} \int^{\frac{1}{2}} \frac{2 n_{1}}{d l^{2}}\left(x+\frac{1}{2}-\frac{\ell}{L}\right) d x=0 \\
& \int_{-\frac{1}{2}}^{\frac{1}{2}}\left(a x+m_{I}\right) d x-\int_{\frac{1}{2}}^{\frac{1}{2}} \frac{2 n_{2}}{d l^{2}}\left(x-\frac{1}{2}+\frac{\ell}{L}\right) d x \\
& +\int^{\frac{\ell}{\mathrm{L}}} \frac{-\frac{1}{2}}{\mathrm{~d} \ell^{2}}\left(\mathrm{x}+\frac{1}{2}-\frac{\ell}{\mathrm{L}}\right) \mathrm{dx}=0 \\
& -\frac{1}{2}
\end{aligned}
$$


and

$$
\int_{-\frac{1}{2}}^{\frac{1}{2}}\left(2 a x+m_{I}+m_{0}\right)\left(\frac{1}{2}+\frac{\ell}{L}-x\right) d x-\int_{\frac{1}{2}}^{\frac{1}{2}} \frac{2 n_{2}}{d \ell^{2}}\left(x-\frac{1}{2}+\frac{\ell}{L}\right)\left(\frac{1}{2}+\frac{\ell}{L}-x\right) d x
$$

$$
+\int_{-\frac{1}{2}}^{\frac{1}{2}} \frac{2\left(\mathrm{n}_{1}+\mathrm{n}_{2}^{\prime}\right)}{\mathrm{d} \ell^{2}}\left(\mathrm{x}+\frac{1}{2}-\frac{\ell}{\mathrm{L}}\right)\left(\frac{1}{2}+\frac{\ell}{\mathrm{L}}-\mathrm{x}\right) \mathrm{dx}=0
$$

Equations Al6 through Al8 contain three unknowns: a, $\mathrm{m}_{\mathrm{o}}$, and $\mathrm{m}_{\mathrm{I}}$. Completing the integrations results in

$$
\begin{gathered}
a=\frac{1}{d L^{2}}\left(3-\frac{2 l}{L}\right)\left(n_{2}-n^{\prime}-n_{1}\right) \\
m_{0}=\frac{1}{d L^{2}} n_{1} \\
m_{I}=\frac{1}{d L^{2}}\left(n_{2}+n_{2}^{\prime}\right)
\end{gathered}
$$

Substitution of Equations A19 through A21 into Equations A14 and A15 leads to

$$
\begin{gathered}
N_{1}(x)=\frac{1}{d^{2}}\left[(3-2 \beta)\left(n_{2}-n_{2}^{\prime}-n_{1}\right) x+n_{1}\right] \text { for } \beta-\frac{1}{2} \leq x \leq \frac{1}{2} \\
N_{1}(x)=\frac{1}{d^{2}}\left\{\left[(3-2 \beta)\left(n_{2}-n_{1}^{\prime}-n_{1}\right)+\frac{2 n_{1}}{\beta^{2}}\right] x+\left(\frac{1-\beta}{\beta}\right)^{2} n_{1}\right\} \\
\text { for }-\frac{1}{2} \leq x \leq \beta-\frac{1}{2}
\end{gathered}
$$


and

$$
\begin{array}{ll}
N_{2}(x)=\frac{1}{d L^{2}}\left\{\left[(3-2 \beta)\left(n_{2}-n_{2}^{\prime}-n_{1}\right)-\frac{2 n_{2}}{\beta^{2}}\right] x+\left(\frac{1-\beta}{\beta}\right)^{2} n_{2}+n_{2}^{\prime}\right\} & \text { for } \frac{1}{2}-\beta \leq x \leq \frac{1}{2} \\
N_{2}(x)=\frac{1}{d L^{2}}\left[(3-2 \beta)\left(n_{2}-n_{2}^{\prime}-n_{1}\right) x+n_{2}+n_{2}^{\prime}\right]
\end{array}
$$$$
\text { for } \beta-\frac{1}{2} \leq x \leq \frac{1}{2}-\beta
$$$$
N_{2}(x)=\frac{1}{d L^{2}}\left\{\left[(3-2 \beta)\left(n_{2}-n_{2}^{\prime}-n_{1}\right)+\frac{2 n_{2}^{\prime}}{\beta^{2}}\right] x+\left(\frac{1-\beta}{\beta}\right)^{2} n_{2}^{\prime}+n_{2}\right\}
$$$$
\text { for }-\frac{1}{2} \leq x \leq \beta-\frac{1}{2}
$$

where

$$
\beta=\frac{\ell}{L}
$$

Note that Equations $\mathrm{Al} 2$ and $\mathrm{Al} 3$ dictate that either $\mathrm{n}_{2}$ or $\mathrm{n}_{2}^{\prime}$ in Equations $\mathrm{A} 22$ and $\mathrm{A} 23$ is zero.

\section{Kinematics of the vehicle}

14. A tracked vehicle in transient motion is shown schematically in Figure $\mathrm{A} 8$. The $\mathrm{XYZ}$ coordinates are the local coordinate systems that are fixed with respect to the moving vehicle (also see Figure A4). The origin 0 of this coordinate system stays, for all time, at a distance $C_{X}$ from the center of gravity of the vehicle. The $\Psi \Phi$ coordinate system is fixed on level ground, and its origin coincides 
with the center of gravity at time zero. The vehicle can maneuver on the $\Psi \Phi$ plane and the displacements of the center of gravity of the vehicle from this reference frame are $\Psi(t)$ and $\Phi(t)$.

15. The velocities $v_{X}$ and $v_{Y}$ (relative to the origin of the $\Psi \Phi$ coordinate system) as well as the velocities $v_{\Psi}$ and $v_{\Phi}$ are related to the instantaneous velocity $v$ of the CG by

$$
v=\sqrt{v_{X}^{2}+v_{Y}^{2}}=\sqrt{v_{\Psi}^{2}+v_{\Phi}^{2}}
$$

The side-slip angle $\alpha$, which is the angle between the velocity vector $v$ and the longitudinal $X$ axis of the vehicle, is related to the velocities $\mathrm{v}_{\mathrm{X}}$ and $\mathrm{v}_{\mathrm{Y}}$ as

$$
\alpha=\tan ^{-1} \frac{v_{Y}}{v_{X}}, \frac{d \alpha}{d t}=\left(v_{X} \frac{d v_{Y}}{d t}-v_{Y} \frac{d v_{X}}{d t}\right) / v^{2}
$$

The yaw angle $\omega$ and the directional angle $\theta$ are related to $\alpha$ as

$$
\theta=\omega-\alpha, \frac{d \theta}{d t}=\frac{d \omega}{d t}-\frac{d \alpha}{d t}
$$

Substitution of Equation A26 into Equation A27 leads to

$$
\frac{d \Theta}{d t}=\frac{d \omega}{d t}-\left(v_{X} \frac{d v_{Y}}{d t}-v_{Y} \frac{d v_{X}}{d t}\right) / v^{2}
$$

16. The radius of curvature of the trajectory of the center of gravity (i.e., the distance between $\mathrm{CR}$ and $\mathrm{CG}$ (Figures A5 and A9)) is

$$
R_{0}=v / \frac{d \Theta}{d t}=\frac{v^{3}}{v^{2} \frac{d \omega}{d t}-v_{X} \frac{d v_{Y}}{d t}+v_{Y} \frac{d v_{X}}{d t}}
$$


The coordinates of the trajectory of the center of gravity of the vehicle can be written as

$$
\left.\begin{array}{l}
\Psi(t)=-\int_{0}^{t} v \cos \theta d t \\
\Phi(t)=\int_{0}^{t} v \sin \theta d t
\end{array}\right\}
$$

17. The coordinates of the instantaneous center of rotation (ICR) of 'the hull in the $\mathrm{XY}$ systems $\left(\mathrm{X}_{\mathrm{I}}, \mathrm{Y}_{\mathrm{I}}\right)$ and the instantaneous radius of curvature $\left(R_{I}\right)$ are (Figures $A 5$ and $A 9$ )

$$
\begin{aligned}
& X_{I}=P+c_{X}=v_{Y} / \frac{d \omega}{d t}+c_{X} \\
& Y_{I}=\tilde{R}=v_{X} / \frac{d \omega}{d t} \\
& R_{I}=\sqrt{\tilde{R}^{2}+P^{2}}
\end{aligned}
$$

The instantaneous velocities of an arbitrary point e of the hull are shown in Figure $\mathrm{A} 9$ and can be written as

$$
\begin{gathered}
v_{e X}=v_{X}+Y \frac{d \omega}{d t} \\
v_{e Y}=v_{Y}-\left(X-c_{X}\right) \frac{d \omega}{d t} \\
v_{e}=\sqrt{\left(v_{X}+Y \frac{d \omega}{d t}\right)^{2}+\left[v_{Y}-\left(X-c_{X}\right) \frac{d \omega}{d t}\right]^{2}}
\end{gathered}
$$


Track slip velocity and displacement

18. Assume that $v_{s 1}\left(v_{s 1}=\dot{\Delta}_{1}\right)$ is the slip velocity of an arbitrary point $e_{1}$ of the outer track and $v_{s 2}\left(v_{s 2}=\dot{\Delta}_{2}\right)$ is the slip velocity at point $e_{2}\left(e_{1}\right.$ and $e_{2}$ have the same abscissa) of the inner track (Figure A5). The $\mathrm{X}$ and $\mathrm{Y}$ components of these velocities are

$$
\left.\begin{array}{c}
v_{s X 1}=C_{1} \frac{d \omega}{d t}=\xi_{1} L \frac{d \omega}{d t} \\
v_{s Y 1}=\left(X-P-C_{X}\right) \frac{d \omega}{d t}=L\left(X-c_{X}\right) \frac{d \omega}{d t}-v_{Y}
\end{array}\right\} \text { For the outer track }
$$

As indicated in Figure Al0, the angular velocity $\mathrm{d} \omega / \mathrm{dt}$ and the value of $\tilde{\mathrm{R}}$ can be written as

$$
\left.\begin{array}{c}
\frac{d \omega}{d t}=\frac{1}{b L}\left(v_{X 1}-v_{s X 1}-v_{X 2}+v_{s X 2}\right) \\
\tilde{R}=\frac{1}{2 \frac{d \omega}{d t}}\left(v_{X 1}-v_{s X 1}+v_{X 2}-v_{s X 2}\right)
\end{array}\right\}
$$

where

$$
\begin{aligned}
& \mathrm{v}_{\mathrm{X} 1}=\text { the velocity of the outer track in } \mathrm{x} \text { direction } \\
& \mathrm{v}_{\mathrm{X} 2}=\text { the velocity of the inner track in } \mathrm{x} \text { direction }
\end{aligned}
$$

The ratio of $\mathrm{v}_{\mathrm{X} 1}$ and $\mathrm{v}_{\mathrm{X} 2}$ is defined as the steering ratio $\varepsilon$. Thus

$$
\varepsilon=\mathrm{v}_{\mathrm{X} 1} / \mathrm{v}_{\mathrm{X} 2}
$$


Substitution of Equations A31 and A36 into Equation A35 leads to

$$
\begin{array}{ll}
v_{s X 1}=\varepsilon v_{X 2}-\left(v_{X}+\frac{b L}{2} \frac{d \omega}{d t}\right) & \text { For the outer track } \\
v_{s X 2}=v_{X 2}-\left(v_{X}-\frac{b L}{2} \frac{d \omega}{d t}\right) & \text { For the inner track }
\end{array}
$$

Comparison between Equations $\mathrm{A} 37$ and $\mathrm{A} 38$ and Equations A33 and A34 results in

$$
\begin{aligned}
& \xi_{1}=\left(\varepsilon v_{X 2}-v_{X}\right) /\left(L \frac{d \omega}{d t}\right)-\frac{b}{2} \\
& \xi_{2}=\left(v_{X 2}-v_{X}\right) /\left(L \frac{d \omega}{d t}\right)+\frac{b}{2}
\end{aligned}
$$

The slip velocities and displacements of the outer and inner tracks can be obtained from Equations A33, A34, and A37. Thus

$$
\begin{aligned}
& \frac{v_{s 1}}{\sqrt{L g}}=\sqrt{\frac{L}{g}} \frac{d \omega}{d t} \sqrt{\xi_{1}^{2}+\left[\left(x-c_{X}\right)-\frac{v_{Y}}{L \frac{d \omega}{d t}}\right]^{2}} \\
& \frac{v_{s 2}}{\sqrt{L g}}=\sqrt{\frac{L}{g} \frac{d \omega}{d t}} \sqrt{\frac{\xi_{2}^{2}+\left[\left(x-c_{X}\right)-\frac{v_{Y}}{L \frac{d \omega}{d t}}\right]^{2}}{\Delta_{1}}} \int_{0}^{t} \frac{v_{s 1}}{L} d t+\frac{\Delta_{I 1}}{L}, \frac{\Delta_{2}}{L}=\int_{0}^{2} \frac{v_{s 2}}{L} d t+\frac{\Delta_{I 2}}{L}
\end{aligned}
$$

where

$$
t_{1}=(L / 2-x) / v_{X 1}
$$




$$
\begin{aligned}
t_{2} & =(L / 2-x) / v_{X 2} \\
\Delta_{I 1} & =\text { initial displacement of the outer track } \\
\Delta_{I 2} & =\text { initial displacement of the inner track }
\end{aligned}
$$

The balance of forces and moments dictates that these initial displacements be numerically equal to $\mathrm{L} f$ ( 6 is the coefficient of rolling resistance, which must be measured experimentally or calculated from empirical relations presented by Rula and Nuttall (1971) for each soil type and each vehicle).

\section{Inertial forces}

19. According to Figure $\mathrm{A} 8$, the relationship between the velocities $v_{\Psi}$ and $v_{\Phi}$ and the velocities $v_{X}$ and $v_{Y}$ can be written as

$$
\left.\begin{array}{l}
\mathrm{v}_{\Psi}=-\mathrm{v}_{\mathrm{X}} \cos \omega-\mathrm{v}_{\mathrm{Y}} \sin \omega \\
\mathrm{v}_{\Phi}=\mathrm{v}_{\mathrm{X}} \sin \omega-\mathrm{v}_{\mathrm{Y}} \cos \omega
\end{array}\right\}
$$

The acceleration in $\Psi$ and $\Phi$ direction, $a_{\psi}$ and $a_{\Phi}$, can be written as

$$
\left.\begin{array}{l}
a_{\Psi}=\frac{d v_{\Psi}}{d t} \\
a_{\Phi}=\frac{d v_{\Phi}}{d t}
\end{array}\right\}
$$

The forward and lateral accelerations, ${ }^{a}{ }_{X}$ and ${ }^{a_{Y}}$, can be written in terms of $a_{\Psi}$ and $a_{\Phi}$ as

$$
\left.\begin{array}{l}
a_{X}=-a_{\Psi} \cos \omega+a_{\Phi} \sin \omega \\
a_{Y}=-a_{\Psi} \sin \omega-a_{\Phi} \cos \omega
\end{array}\right\}
$$


Substitution of Equations $\mathrm{A} 41$ and $\mathrm{A} 42$ into Equation $\mathrm{A} 43$ leads to

$$
\left.\begin{array}{l}
a_{X}=\frac{d v_{X}}{d t}+v_{Y} \frac{d \omega}{d t} \\
a_{Y}=\frac{d v_{Y}}{d t}-v_{X} \frac{d \omega}{d t}
\end{array}\right\}
$$

Hence, the $\mathrm{X}$ and $\mathrm{Y}$ components of the inertial force can be written as

and

$$
\left.\begin{array}{l}
F_{C X}=\frac{W}{g} a_{X}=\frac{W}{g}\left(\frac{d v_{X}}{d t}+v_{Y} \frac{d \omega}{d t}\right) \\
F_{C Y}=\frac{W}{g} a_{Y}=\frac{W}{g}\left(\frac{d v_{Y}}{d t}-v_{X} \frac{d \omega}{d t}\right)
\end{array}\right\}
$$

The rolling resistance

20. The rolling resistance $R_{S}$ is a function of terrain type, vehicle speed, track condition, etc. Therefore, rolling resistance should be measured for every specific condition. In this formulation, however, the rolling resistance is assumed to be proportional to normal load. Thus

$$
R_{S}=\frac{W}{d L^{2}} 6 \int_{-\frac{1}{2}}^{\frac{1}{2}}\left[r_{1}(x)+r_{2}(x)\right] d x
$$

\section{Equation of motion}

21. Steerability and stability of tracked vehicles depend on the dynamic balance between all forces and moments applied on the vehicle. According to Figure $\mathrm{A} 4$, the following three equations govern the motion of the vehicle: 


$$
\begin{aligned}
& \int_{-\frac{1}{2}}^{\frac{1}{2}}\left[t_{1}(x)+t_{2}(x)\right] d x-6 \int_{-\frac{1}{2}}^{\frac{1}{2}}\left[r_{1}(x)+r_{2}(x)\right] d x=f_{C X} \\
& \int_{-\frac{1}{2}}^{\frac{1}{2}}\left[q_{1}(x)+q_{2}(x)\right] d x=f_{C Y} \\
& -\int_{-\frac{1}{2}}^{\frac{1}{2}}\left[q_{1}(x)+q_{2}(x)\right]\left(x-c_{x}\right) d x+\frac{b}{2} \int_{-\frac{1}{2}}^{\frac{1}{2}}\left[t_{1}(x)-t_{2}(x)\right] d x \\
& +\frac{b}{2} 6 \int_{-\frac{1}{2}}^{\frac{1}{2}}\left[r_{2}(x)-r_{1}(x)\right] d x=\frac{I_{2}}{L W} \frac{d^{2} \omega}{d t^{2}}
\end{aligned}
$$

where

$$
\begin{aligned}
& t_{1}(x)=\frac{d^{2}}{W} T_{1}(x) \\
& t_{2}(x)=\frac{d^{2}}{W} T_{2}(x) \\
& q_{1}(x)=\frac{d^{2}}{W} Q_{1}(x) \\
& q_{2}(x)=\frac{d^{2}}{W} Q_{2}(x) \\
& f_{C X}=\frac{F_{C X}}{W} \\
& f_{C Y}=\frac{F}{W} \\
& I_{2}=\text { mass moment of inertia about an axis passing through the } \\
& C G \text { of the vehicle and parallel to the } Z \text { axis (Figure A4) }
\end{aligned}
$$


Equations A47 through A49 with the aid of Equations A7 through A46 constitute three equations that involve three unknowns. The three unknowns are either $\mathrm{v}_{\mathrm{X}}, \mathrm{v}_{\mathrm{Y}}$, and $\mathrm{d} \omega / \mathrm{dt}$ or $\xi_{1}, \xi_{2}$, and $\mathrm{p}$. In order to obtain a complete solution for either of the two sets of unknowns, one of the following driving conditions must be specified: (a) time history of the steering ratio $\varepsilon(t)$ and the initial speed of the vehicle, (b) time history of the velocity of the individual tracks $\mathrm{v}_{\mathrm{X} 1}(\mathrm{t})$ and $\mathrm{v}_{\mathrm{X} 2}(\mathrm{t})$ and the initial speed of the vehicle, (c) time history of the velocity of the vehicle $v(t)$ and the trajectory of motion, (d) time history of the velocity of the vehicle and a constant value of steering ratio $\varepsilon$, or (e) the trajectory of motion and a determination of the maximum velocity time history at which the vehicle can traverse the specified trajectory. A computer program called AGIL was developed to solve Equations A47 through A49 using Newton's iteration technique.

\section{Treatment of sloping terrain}

22. The analysis of the nonuniform turning motion on sloping terrain presented in this section is an extension of the corresponding analysis for steady-state motion on sloping terrain reported by Baladi and Rohani (1979 and 1981). Figure All shows schematically a tracked vehicle under nonuniform (transient) turning motion on a terrain with slope angle $\eta$. In this case, the weight of the vehicle $W$ could be resolved into a normal component (normal to the terrain) $W_{N}$ and a parallel component $\mathrm{W}_{\mathrm{T}}$. Thus

$$
\left.\begin{array}{l}
\mathrm{W}_{\mathrm{N}}=\mathrm{W} \cos n \\
\mathrm{~W}_{\mathrm{T}}=\mathrm{W} \sin \mathrm{n}
\end{array}\right\}
$$

In general, the longitudinal axis of the vehicle $X$ makes an angle $X$ with the component $\mathrm{W}_{\mathrm{T}}$ (Figure A11). Therefore, the component $\mathrm{W}_{\mathrm{T}}$ could be resolved into two components. The first component $W_{T X}$ is 
parallel to the $X$ axis of the vehicle and the second component $W_{T Y}$ is parallel to the $\mathrm{Y}$ axis. Thus

$$
\left.\begin{array}{l}
\mathrm{w}_{\mathrm{TX}}=\mathrm{w}_{\mathrm{T}} \cos \mathrm{x}=\mathrm{W} \sin \mathrm{n} \cos \mathrm{X} \\
\mathrm{W}_{\mathrm{TY}}=\mathrm{W}_{\mathrm{T}} \sin \mathrm{x}=\mathrm{W} \sin \mathrm{n} \sin \mathrm{x}
\end{array}\right\}
$$

The angle $X$ is related to the yaw angle $\omega$ through the following relation

$$
x=w+v
$$

where $v$ is a constant. The numerical value of $v$ depends on the initial position of the vehicle $(\nu=0,90,180$, and 270 deg corresponds, respectively, to the initial position of the vehicle at points $1,2,3$, and 4 in Figure Al1). Substitution of Equation A52 into Equation A51 leads to

$$
\left.\begin{array}{l}
\mathrm{W}_{\mathrm{TX}}=\mathrm{W} \sin n \cos (\omega+v) \\
\mathrm{W}_{\mathrm{TY}}=\mathrm{W} \sin n \sin (\omega+\nu)
\end{array}\right\}
$$

In view of Equations $\mathrm{A} 50$ and $\mathrm{A} 53$, the normal stresses under the outer and inner tracks (Equations $\mathrm{A} 5$ and $\mathrm{A} 6$ ) become

$$
\begin{aligned}
R_{1}(x)=\frac{W}{d L^{2}}\left\{\frac{\cos \eta}{2}\right. & +6 x c_{x} \cos \eta-\frac{h}{b}\left[\frac{F_{C Y}}{W}-\sin \eta \sin (\omega+v)\right] \\
& \left.-6 h x\left[\frac{F C X}{W}+\sin \eta \cos (\omega+v)\right]+\frac{D_{L N}(x)}{W}\right\}
\end{aligned}
$$




$$
\begin{aligned}
R_{2}(x)=\frac{W}{d L^{2}}\left\{\frac{\cos \eta}{2}\right. & +6 x c_{X} \cos \eta+\frac{h}{b}\left[\frac{F C Y}{W}-\sin \eta \sin (\omega+v)\right] \\
& \left.-6 h x\left[\frac{F C X}{W}+\sin \eta \cos (\omega+v)\right]+\frac{\mathrm{DLN}_{2}(x)}{W}\right\}
\end{aligned}
$$

Equations A54 and A55 can be combined with Equations A7 through A46 to develop the equations of motion for a sloping terrain. Thus

$$
\begin{aligned}
\int_{-\frac{1}{2}}^{\frac{1}{2}}\left[t_{1}(x)+t_{2}(x)\right] d x & -6 \int_{-\frac{1}{2}}^{\frac{1}{2}}\left[r_{1}(x)+r_{2}(x)\right] d x \\
& =f_{C X}+\sin \eta \cos (\omega+v)
\end{aligned}
$$

$$
\int_{-\frac{1}{2}}^{\frac{1}{2}}\left[q_{1}(x)+q_{2}(x)\right] d x=f_{C Y}-\sin \eta \sin (\omega+v)
$$

$$
\int_{-\frac{1}{2}}^{\frac{1}{2}}\left[q_{1}(x)+q_{2}(x)\right]\left(x-c_{x}\right) d x+\frac{b}{2} \int_{-\frac{1}{2}}^{\frac{1}{2}}\left[t_{2}(x)-t_{1}(x)\right] d x
$$

$$
+\frac{b}{2} 6 \int_{-\frac{1}{2}}^{\frac{1}{2}}\left[r_{1}(x)-r_{2}(x)\right] d x=\frac{I_{z}}{L W} \frac{d^{2} W}{d t^{2}}
$$




\section{Sprocket power}

23. The steering performance of a tracked vehicle may be limited either by its stability or by the power available at the sprockets. The powers that must be available at the inner and outer track sprockets, PT1 and PT2, respectively, are

$$
\begin{gathered}
\frac{\mathrm{PT} 1}{\mathrm{~W} \sqrt{\mathrm{Lg}}}=\frac{\mathrm{L}}{\sqrt{\mathrm{Lg}}} \int_{-\frac{1}{2}}^{\frac{1}{2}}\left[\mathrm{t}_{1}(\mathrm{x}) \dot{\delta}_{1} / \cos \gamma_{1}\right] \mathrm{dx} \\
+6 \cos n \frac{\mathrm{v}_{\mathrm{X} 1}}{\sqrt{\mathrm{Lg}}} \frac{\int_{1}^{\frac{1}{2}} \mathrm{r}_{1}(\mathrm{x}) \mathrm{dx}}{\frac{\frac{1}{2}}{\int^{2}}\left[\mathrm{r}_{1}(\mathrm{x})+\mathrm{r}_{2}(\mathrm{x})\right] \mathrm{dx}}
\end{gathered}
$$

$$
\frac{\mathrm{PT} 2}{W \sqrt{\mathrm{Lg}}}=\frac{\mathrm{L}}{\sqrt{\mathrm{Lg}}} \int_{-\frac{1}{2}}^{\frac{1}{2}}\left[\mathrm{t}_{2}(\mathrm{x}) \dot{\delta}_{2} / \cos \gamma_{2}\right] d x
$$

$$
+f \cos n \frac{v_{x 2}}{\sqrt{L g}} \frac{\int_{-\frac{1}{2}}^{\frac{1}{2}} r_{2}(x) d x}{\int_{-\frac{1}{2}}^{\frac{1}{2}}\left[r_{1}(x)+r_{2}(x)\right] d x}
$$


Therefore, the total power PT and the differential power PTD required are

$$
\left.\begin{array}{c}
\mathrm{PT}=\mathrm{PT} 1+\mathrm{PT} 2 \\
\mathrm{PTD}=\mathrm{PT} 1-\mathrm{PT} 2
\end{array}\right\}
$$




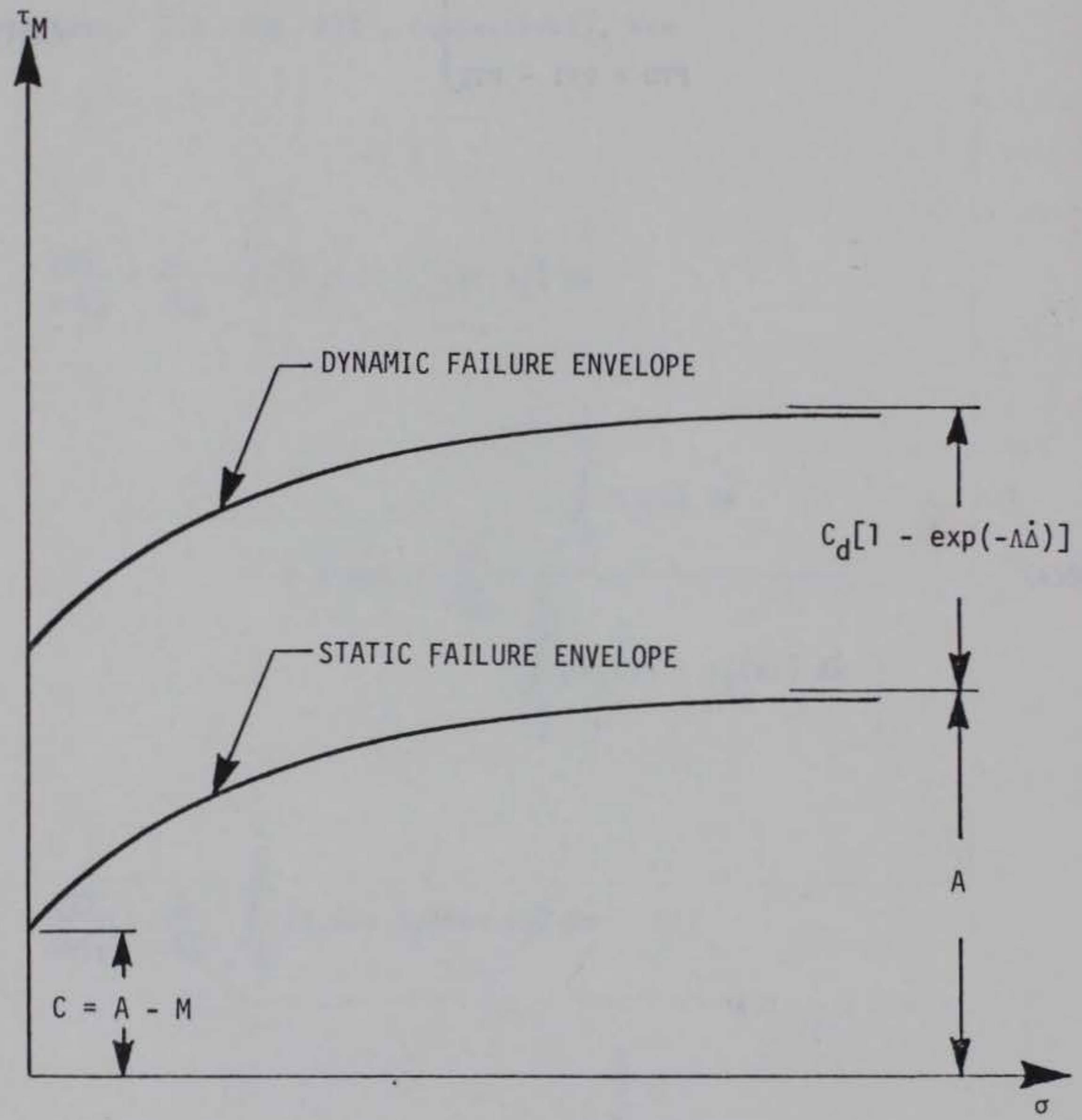

Figure Al. Proposed failure relation for soil 


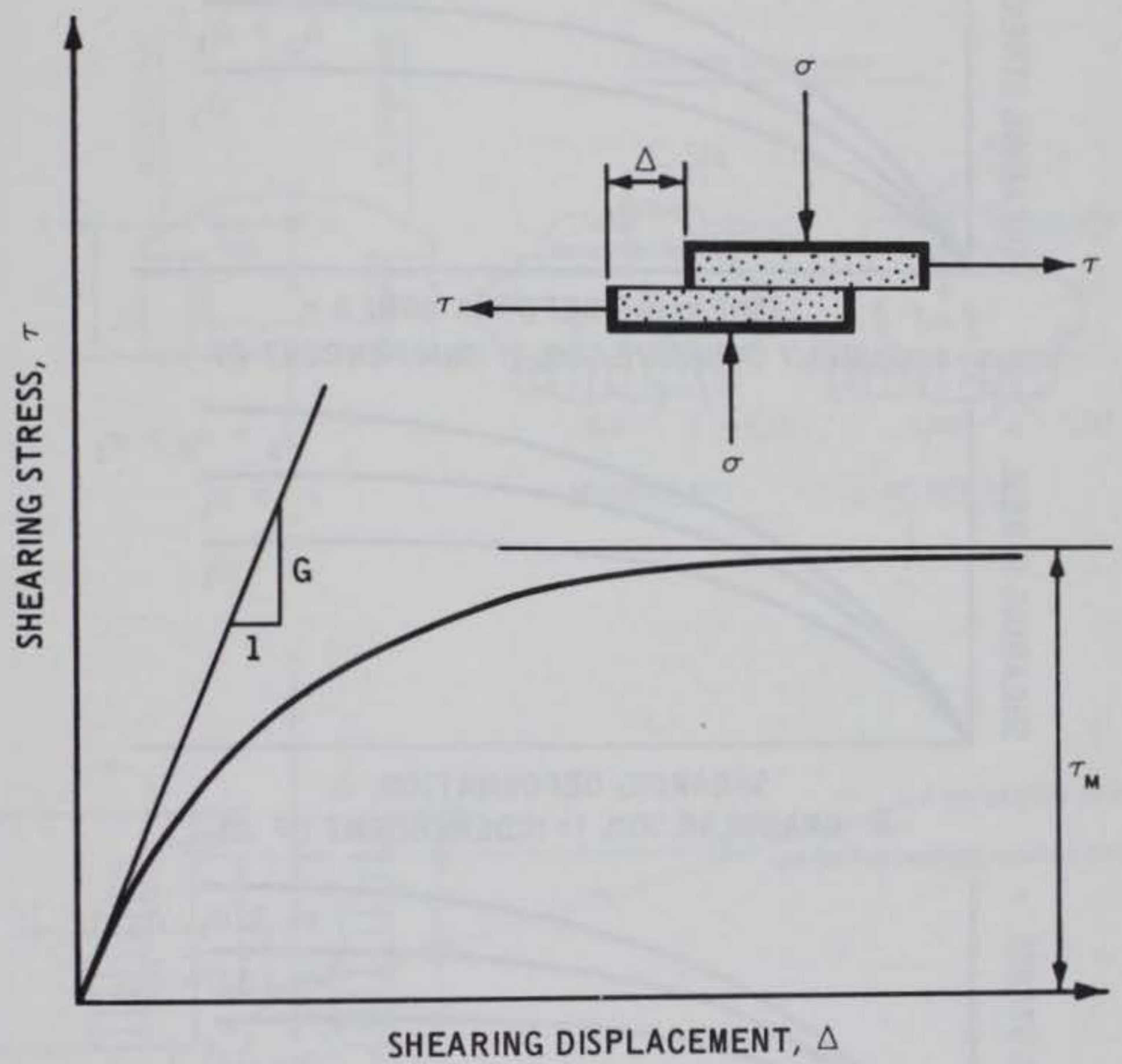

Figure A2. Proposed shear stress/deformation relation during shearing process (Equation A3) 


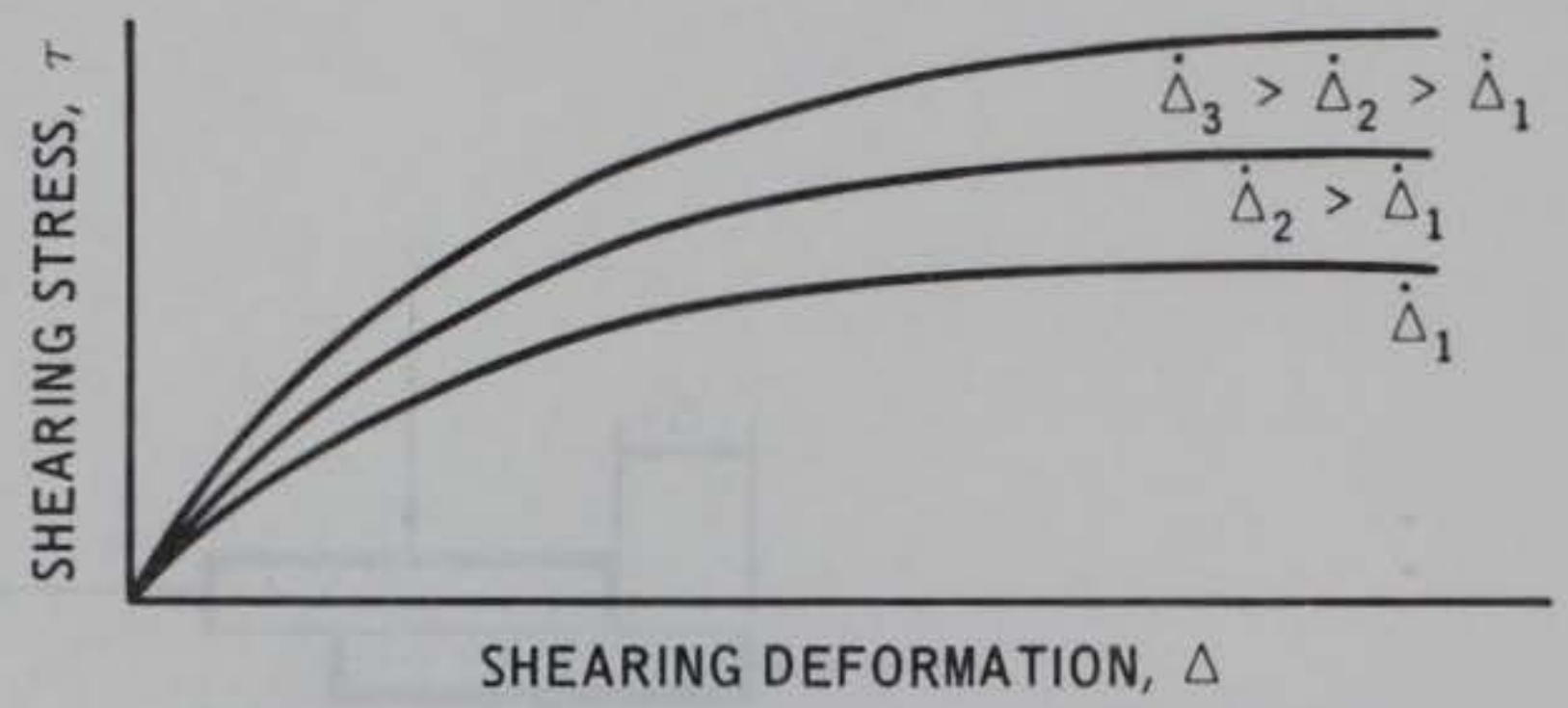

a. PURELY COHESIVE SOIL ( $\tau$ INDEPENDENT OF $\sigma$ )

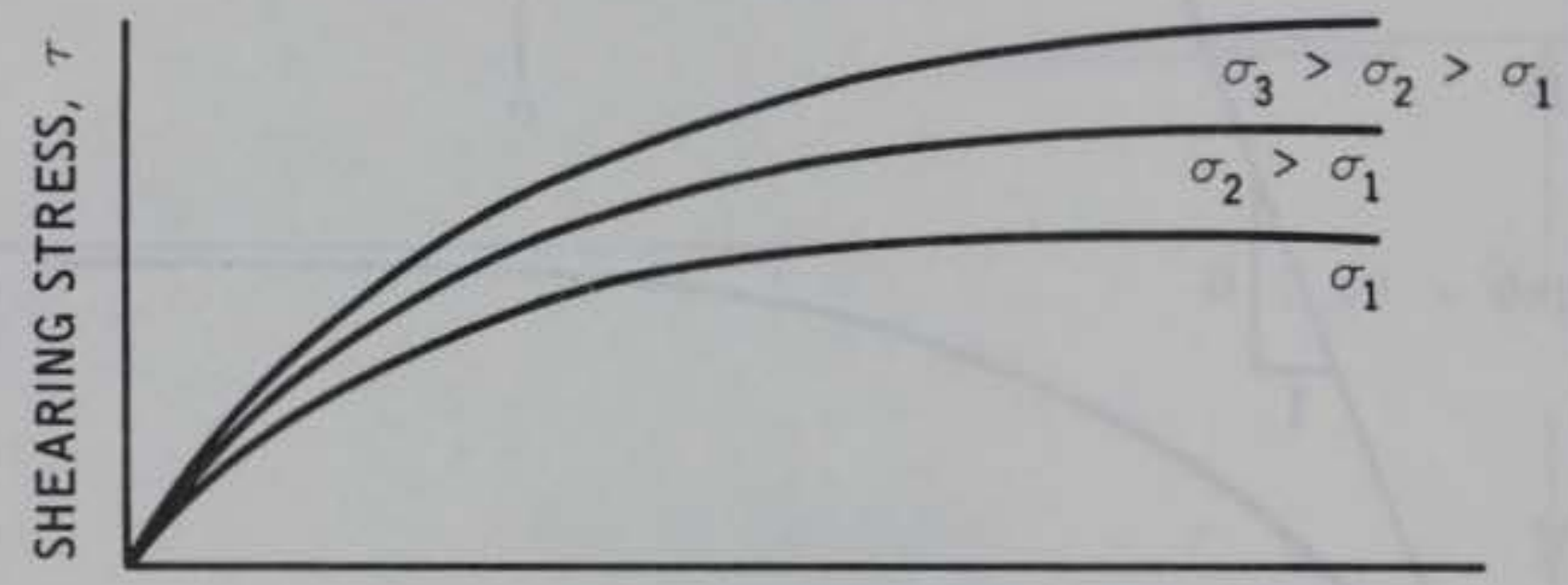

SHEARING DEFORMATION, $\triangle$

b. GRANULAR SOIL ( $\tau$ INDEPENDENT OF $\dot{\Delta}$ )

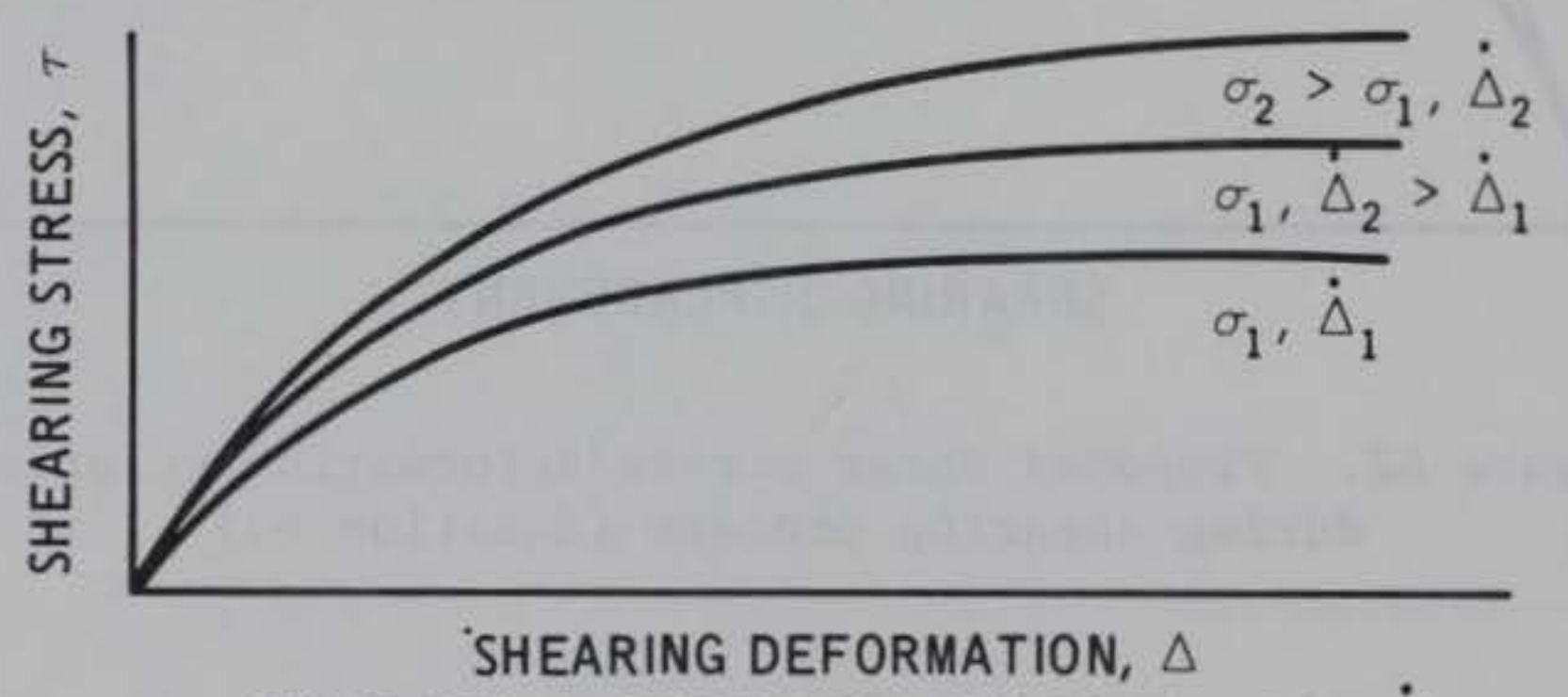

c. MIXED SOIL ( $\tau$ DEPENDENT ON BOTH $\sigma$ AND $\dot{\Delta}$ )

Figure A3. Qualitative behavior of the soil model (Equation 4) for various types of soil 

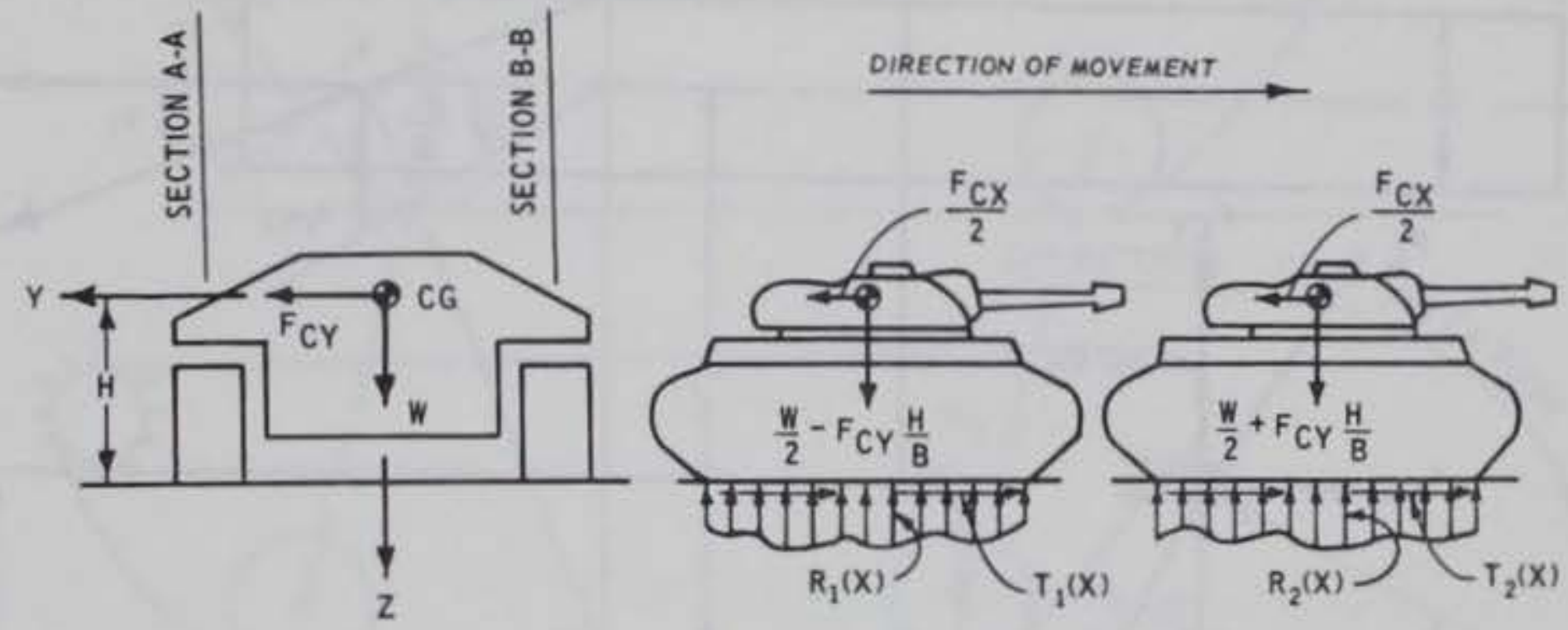

SECTION A-A

SECTION B-B

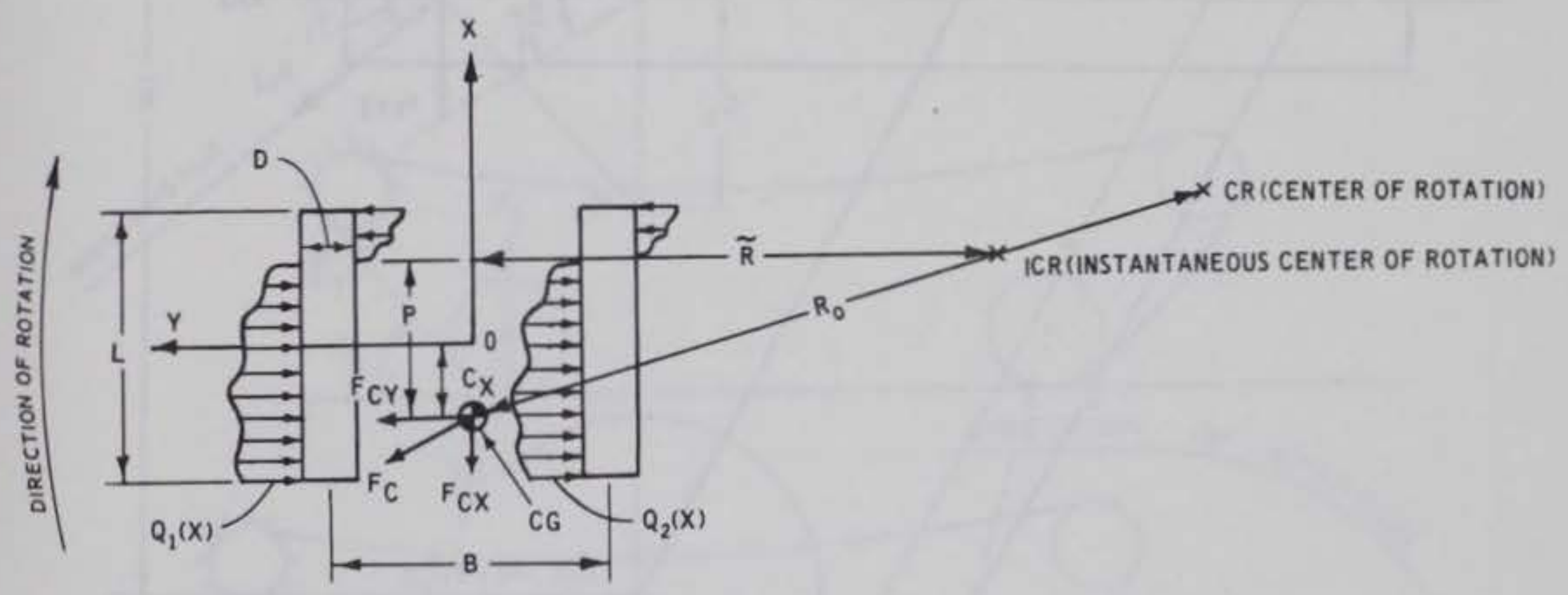

Figure A4. Geometry and boundary conditions of the terrain-vehicle model 


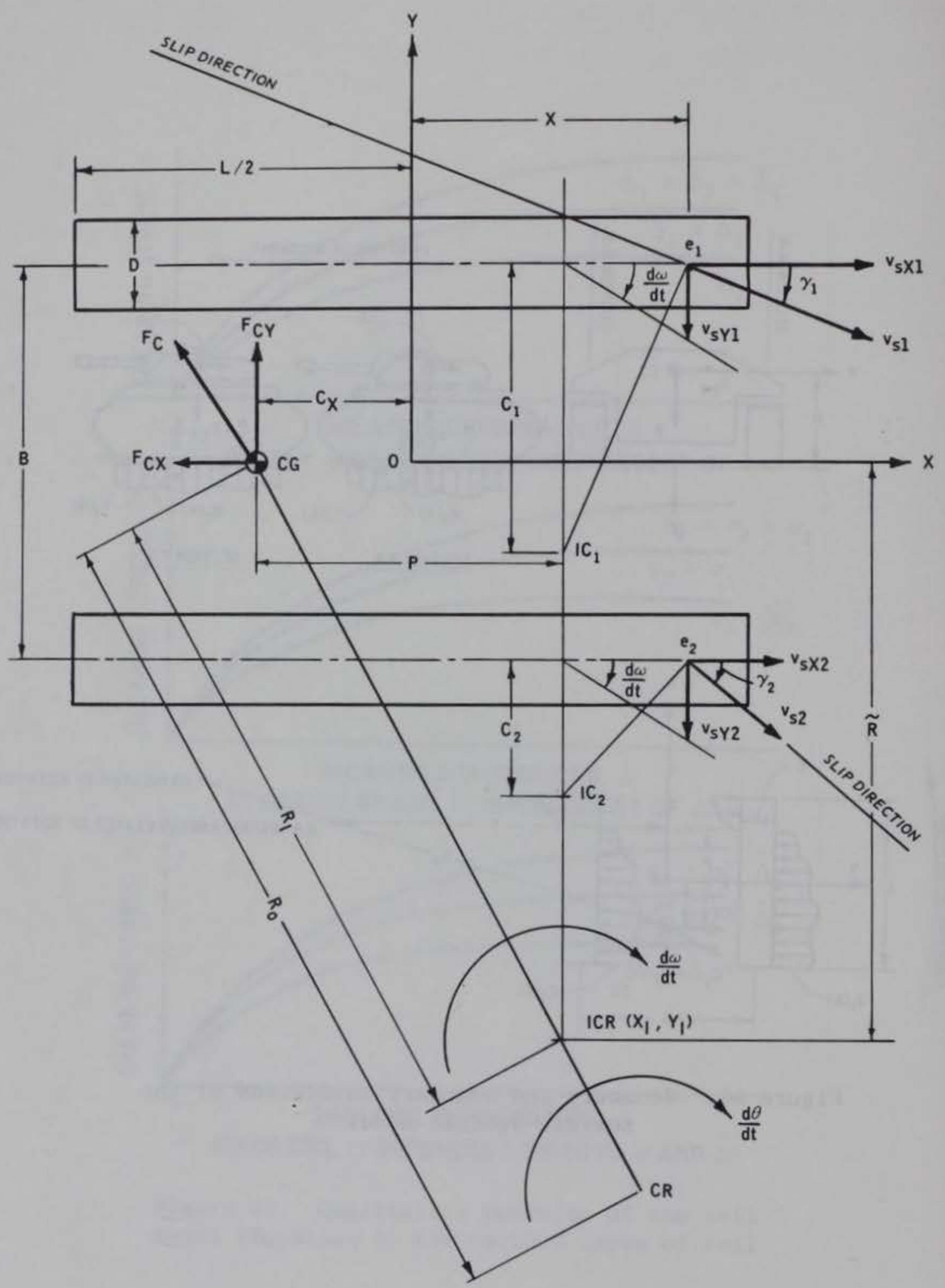

Figure A5. S1ip velocity of track at distance $\mathrm{X}$ from the center of geometry 


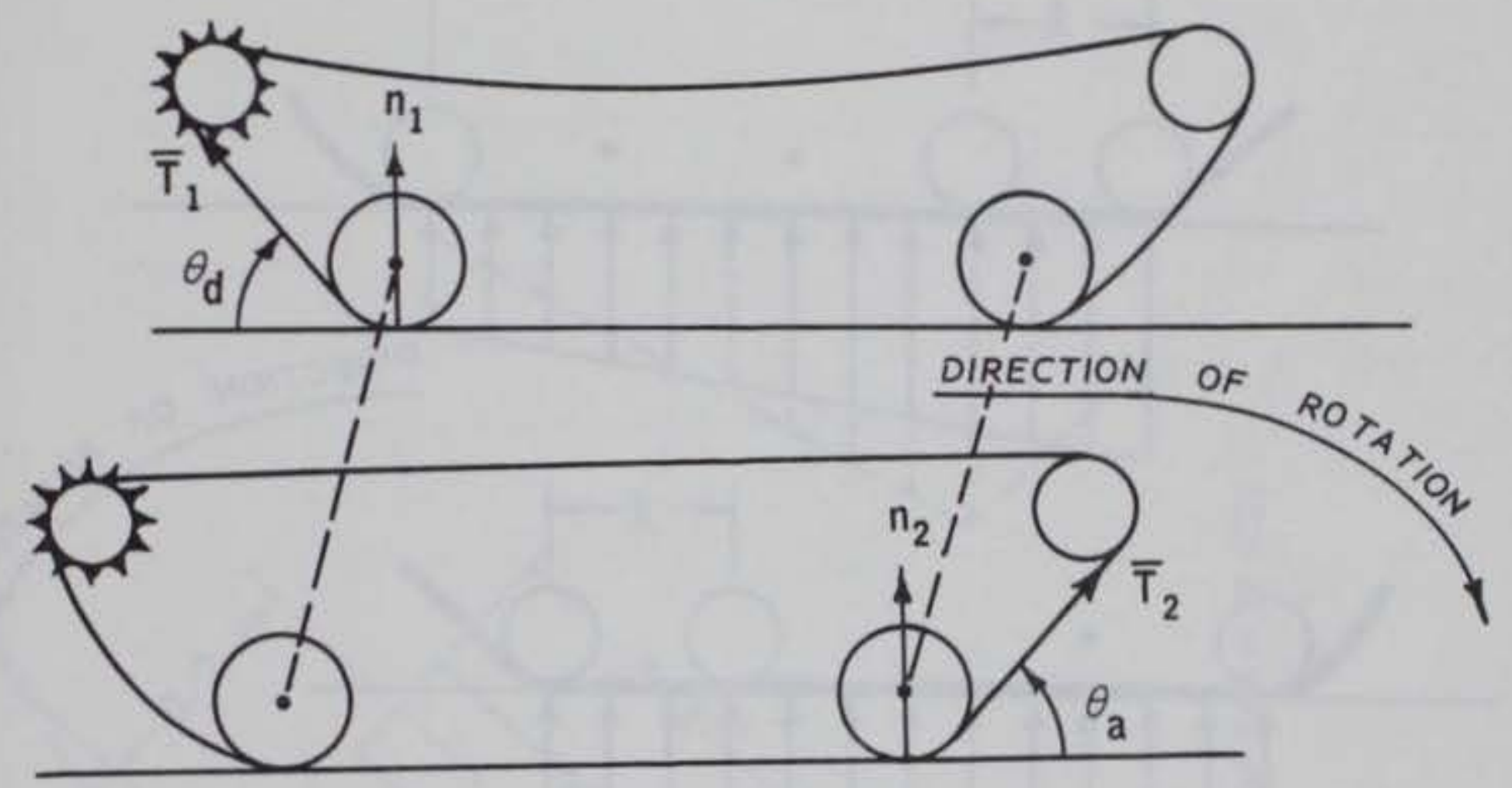

a. LOW SPEED

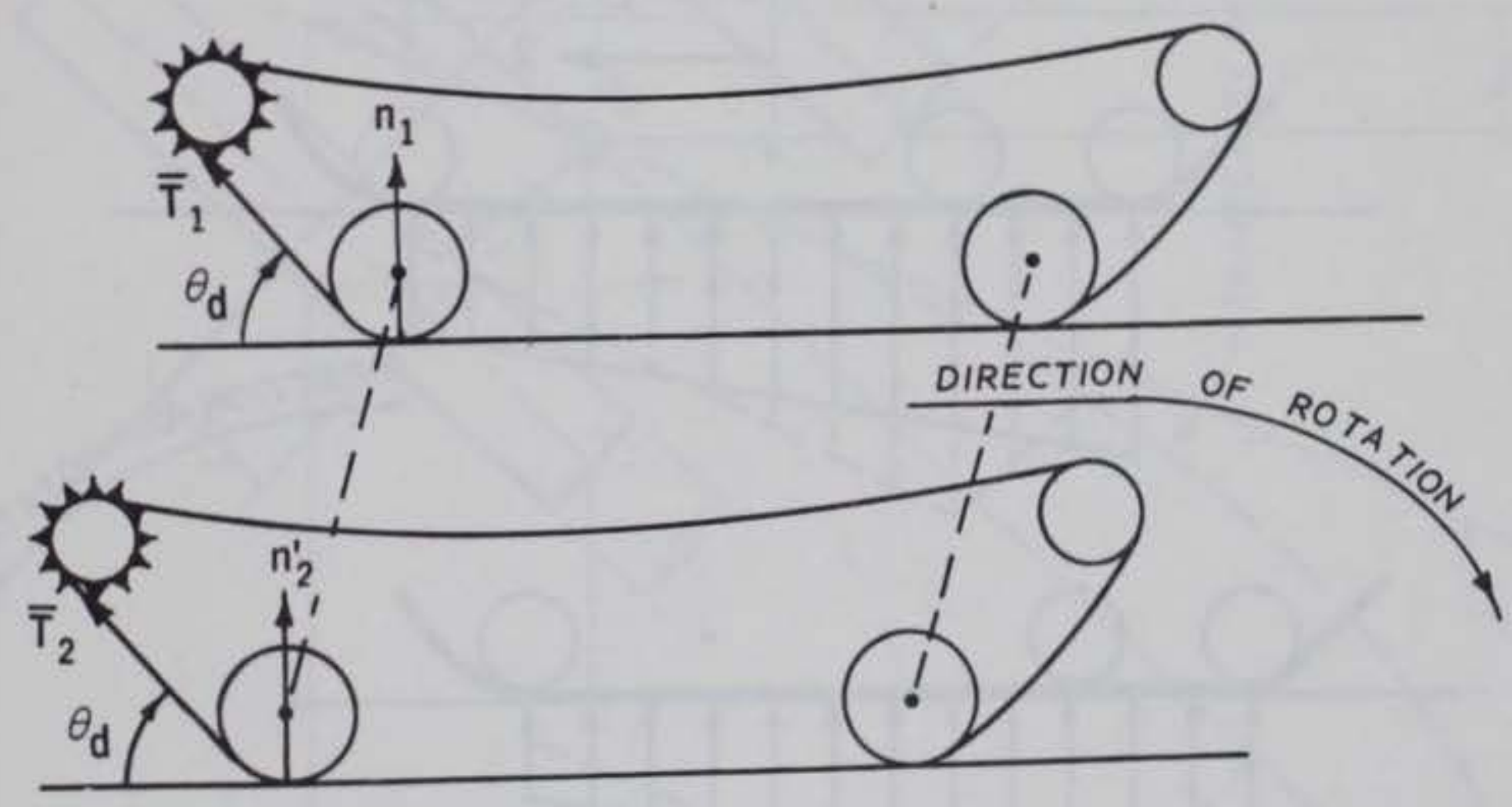

b. HIGH SPEED

Figure A6. Track tension at low and high speeds 
NORMAL STRESS DISTRIBUTION WITHOUT TRACK TENSION

- - - NORMAL STRESS DISTRIBUTION WITH TRACK TENSION

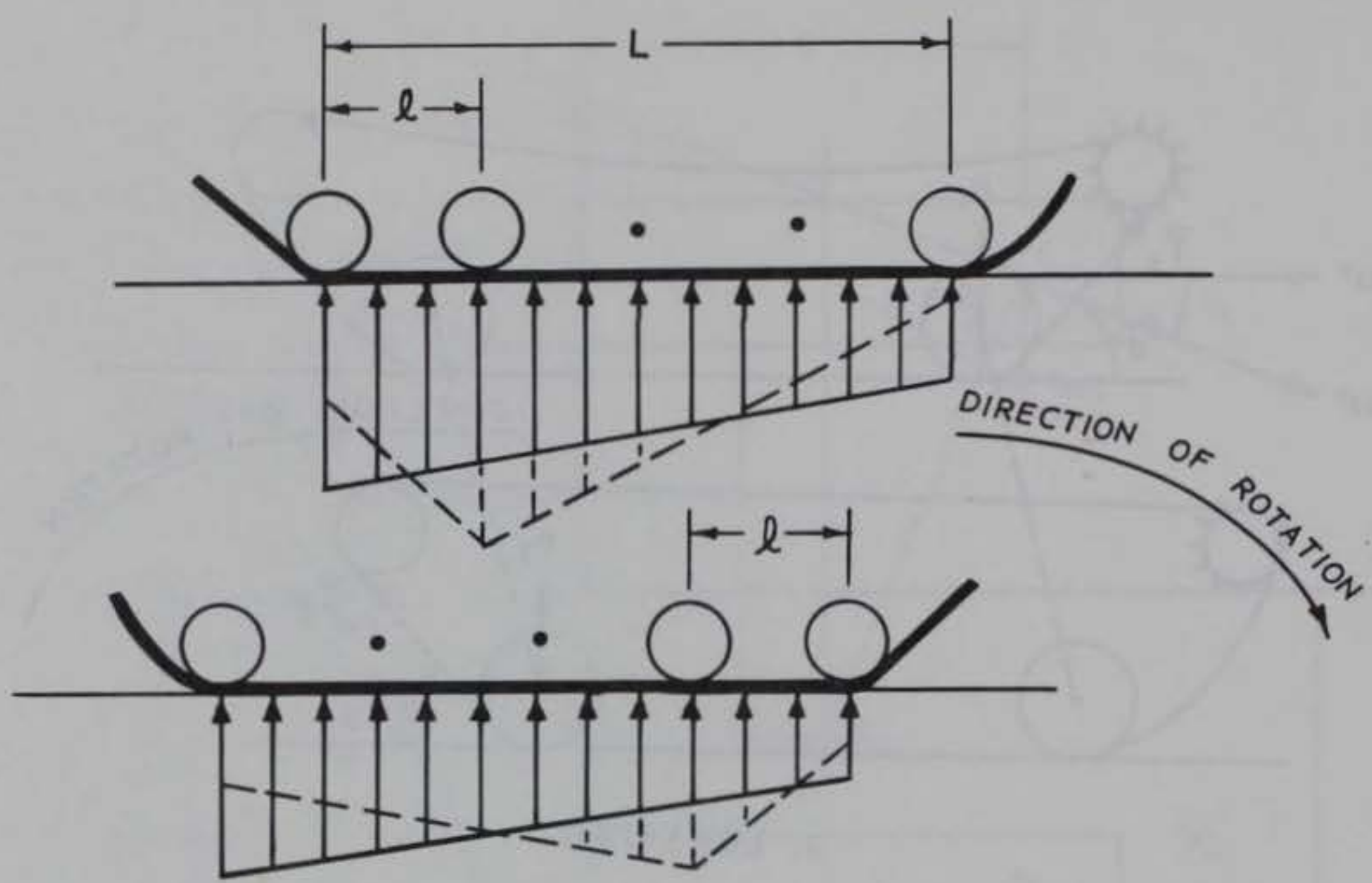

a. LOW SPEED

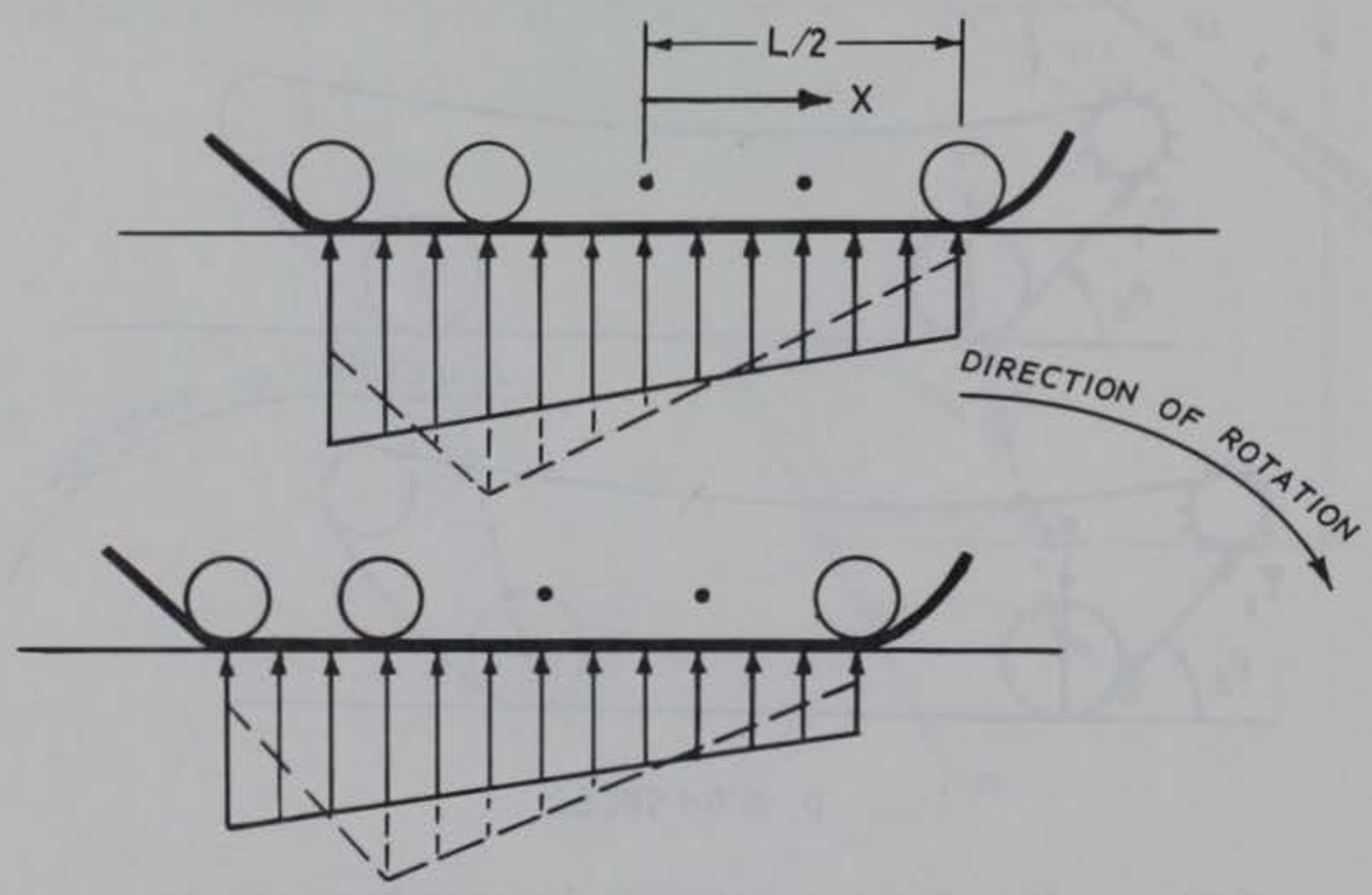

b. HIGH SPEED

Figure A7. Effect of track tension on normal stress distribution 


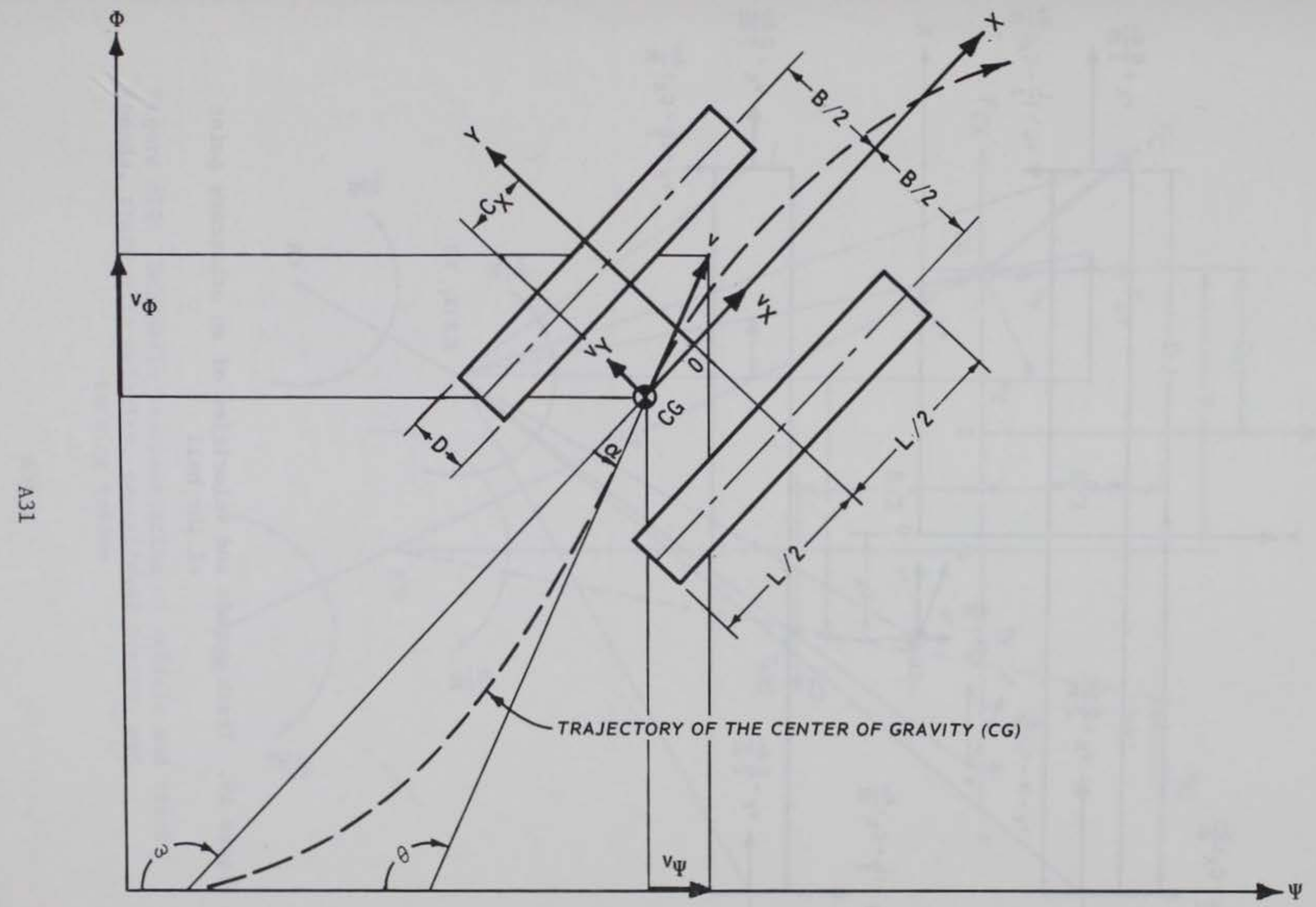

Figure A8. Tracked vehicle in transient motion 


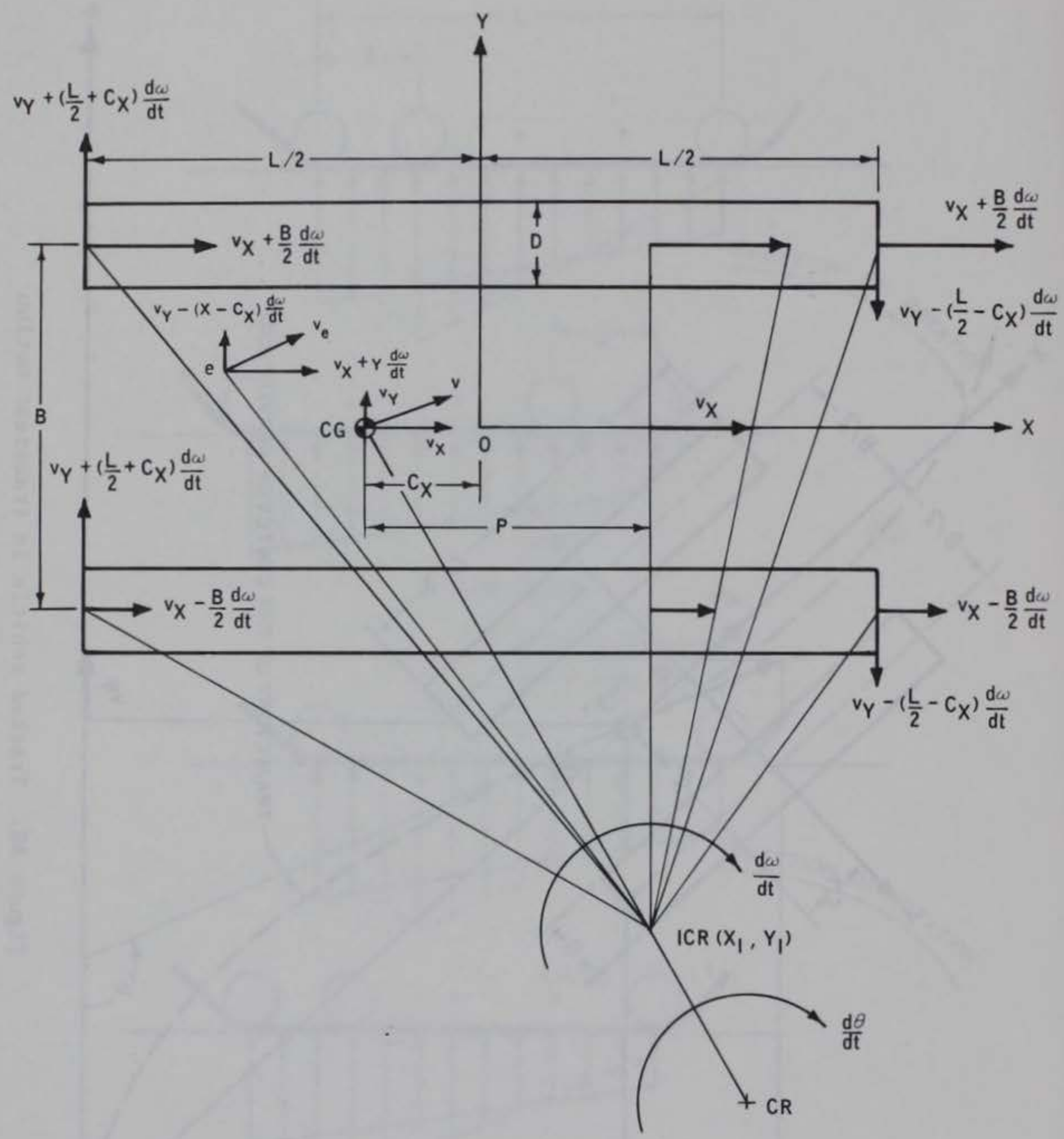

Figure A9. Track speeds and velocities of an arbitrary point of the hull 


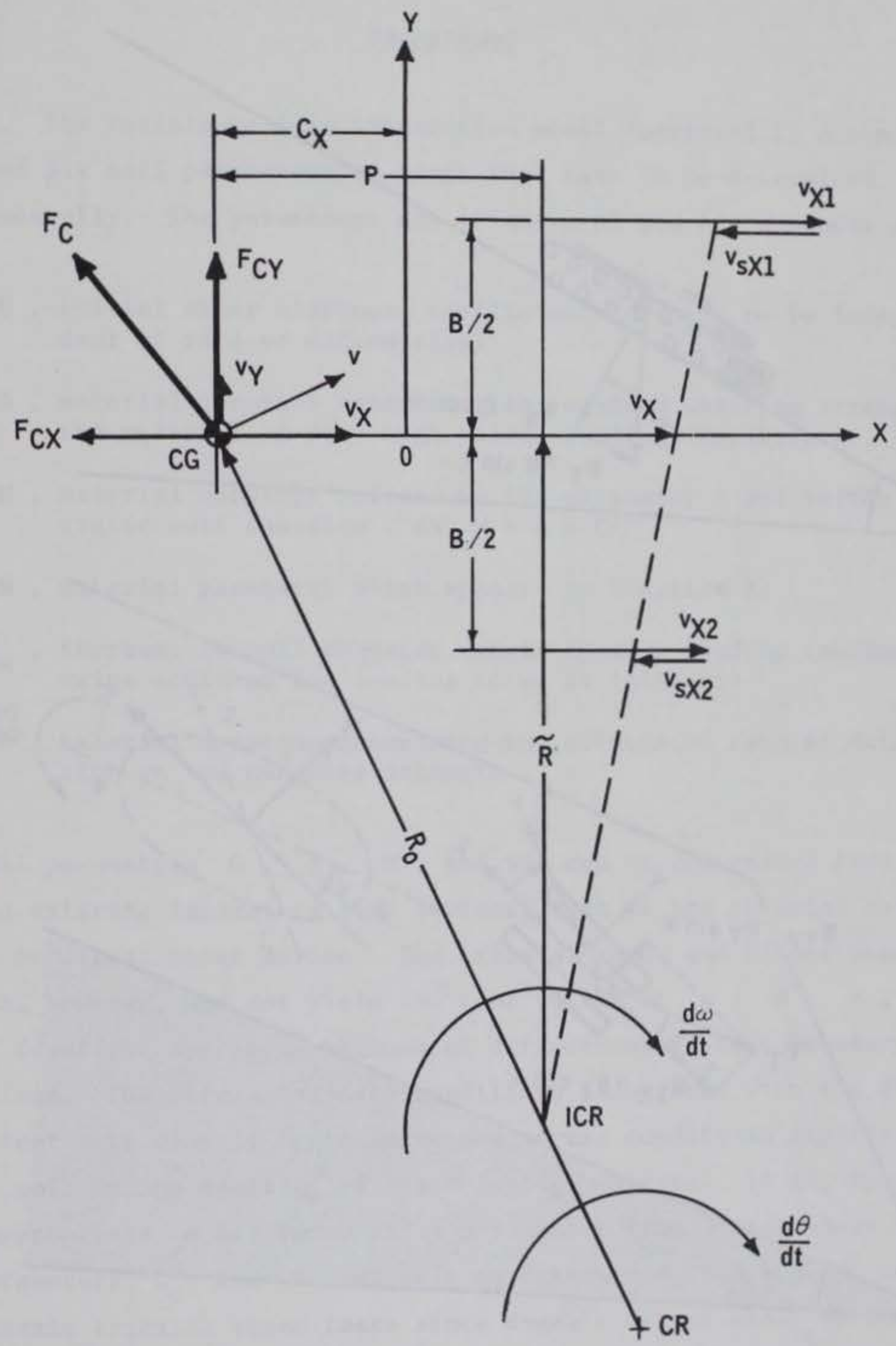

Figure A10. Schematic representation of vehicle and track speeds, track slip velocity, centrifugal forces, and turning radius 

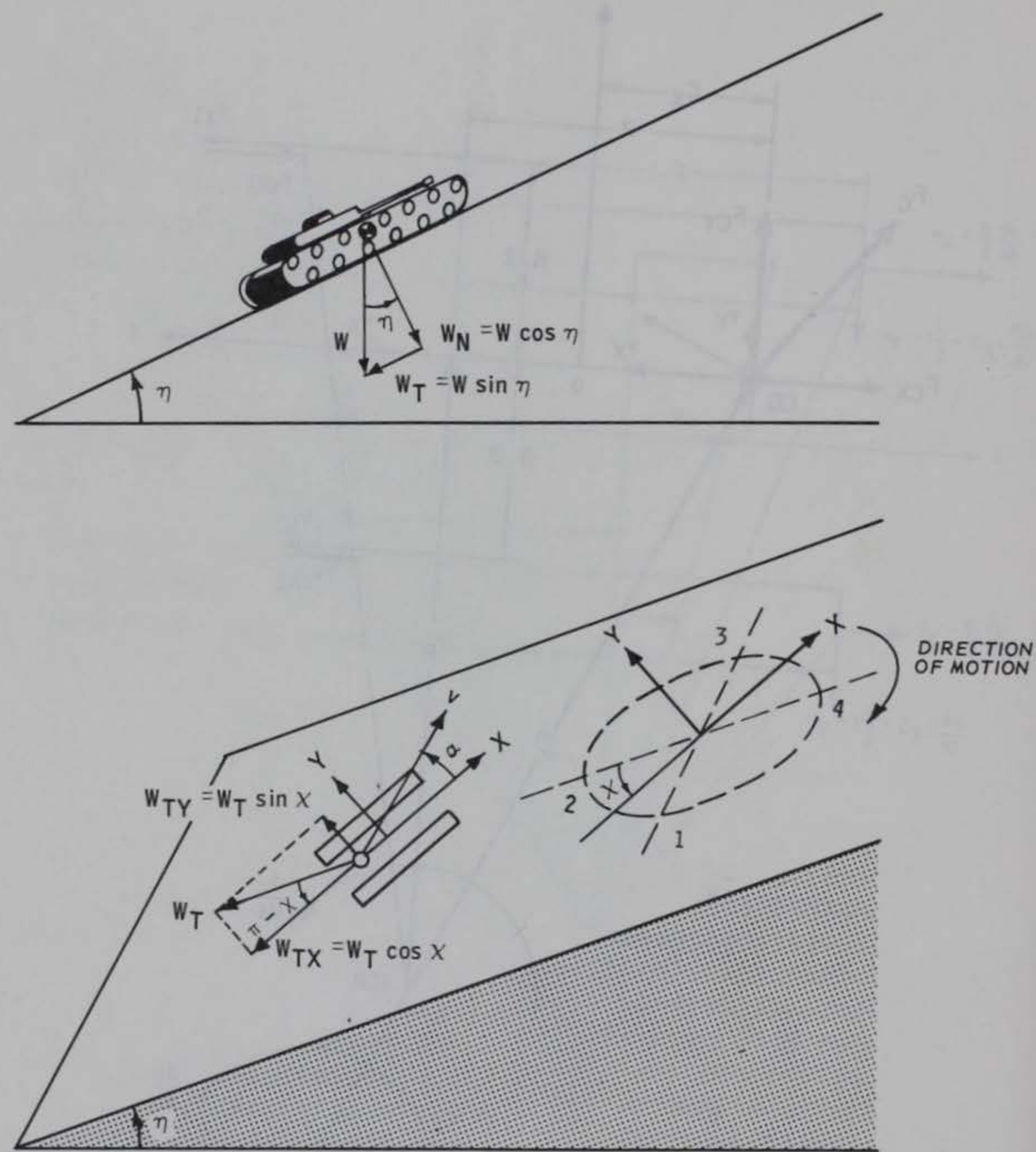

Figure All. Effect of sloping terrain on transient motion 


\section{Background}

1. The terrain-vehicle interaction model described in Appendix A required six soil parameters as input that have to be determined experimentally. The parameters are (Figures $\mathrm{A} 1$ and $\mathrm{A} 2$, Appendix A):

G , initial shear stiffness coefficient (assumed to be independent of rate of deformation)

A , material constant describing the maximum shearing strength of the material at very high normal loading (Equation Al)

$M$, material constant related to the parameter $A$ and to the static soil cohesion $\mathrm{C}$ as $\mathrm{M}=\mathrm{A}-\mathrm{C}$

$\mathrm{N}$, material parameter which appears in Equation $\mathrm{Al}$

$\mathrm{C}_{\mathrm{d}}$, increase in soil cohesion due to dynamic loading (maximum value achieved for loading rates of interest)

$\Lambda$, material constant describing the effects of rate of deformation on the cohesive strength

The soil parameters G, A, M, and N can be determined from various existing laboratory test devices, such as the triaxial shear device or direct shear device. The triaxial shear and direct shear devices, however, may not yield the same values of $\mathrm{G}, \mathrm{A}, \mathrm{M}$, and $\mathrm{N}$ for identical specimens because of differences in test boundary conditions. The stress boundary conditions associated with the direct shear test more closely approximate the stress conditions experienced by the soil during steering of track-laying vehicles. It is, therefore, more appropriate to determine these parameters from direct shear tests. The parameters $\mathrm{C}_{\mathrm{d}}$ and $\Lambda$ can only be determined from special static and dynamic triaxial shear tests since dynamic direct shear devices are not presently available. Therefore, to adequately determine the five soil parameters, two separate test series may be required: 
a. Direct shear tests to define G, A, M, and N .

b. Static and dynamic triaxial shear tests to define $C_{d}$ and $\Lambda$.

It should be noted that in determining $C_{d}$ and $\Lambda$ from triaxial tests rather than direct shear tests, it is assumed that these parameters are not sensitive to test boundary conditions. The validity of this assumption should, of course, be evaluated.

2. The most important consideration in conducting laboratory soil tests is that the undisturbed specimens be representative of the materials over which the vehicle must travel. This fact implies that the upper several inches of surface material must be sampled, trimmed to necessary specimen size, and tested in the laboratory. Water content, soil structure, density, and vegetation root systems, all of which affect material response, must be preserved. With this in mind, a field-operated direct shear device capable of testing a variety of in situ surface soils for normal loads of interest was designed and fabricated. The description of the device and the procedure by which the soil parameters can be determined are documented in this appendix.

\section{Direct Shear Device}

\section{Design consideration}

3. Previously proposed field devices were considered but rejected because of one or more of the following reasons: (a) some of the soil parameters could not be measured and hence required additional tests; (b) the necessary support equipment was too massive to be easily field transportable; or (c) specimen disturbances were encountered before testing. The idea of creating a new type of test was also rejected because any new device would contain inherent boundary problems, all of which would have to be evaluated with time and usage. The direct shear device, on the other hand, has been used extensively, and it is a fairly simple test to run. Furthermore, the four basic soil parameters ( $G$, A, M, and N) could be measured rather directly from this 
test. Figure Bl shows a sketch of the field device that was fabricated as a result of this project. Figure B2 represents photographs taken of the device during the conduct of actual field tests.

Specimen container

4. Specimen configuration was the first consideration made in the design of the device. It was assumed that in many cases the in situ soil could not be sampled without disturbance; therefore, the specimen container would have to be placed around the soil. A round ring similar to a coring device would afford the least chance of soil disturbance. However, the stress distribution along a horizontal plane of a circular specimen is not uniform. To reduce the nonuniformity, a square-shaped specimen container was selected.

5. A 4- by 4-in. box was selected in order to keep the shear and normal loads within limits of interest to the analysis of track-laying vehicles and at the same time retain a reasonably large specimen size. The use of deadweights is the simplest way to produce normal load, but use of more than $200 \mathrm{lb}$ in weights is awkward for field testing. Therefore, with the weight requirement below the 200-1b 1imit, normal stress of up to 12 psi can be produced on a 4- by 4-in., or 16-sqin., specimen. However, the largest particle or grain size permissible with a $4-$ by $4-i n$. specimen is probably $1 / 2 \mathrm{in.}$, which is a reasonable limit for most terrains of interest.

6. The overall specimen height was controlled by the depth of the desired shear plane as directed by grouser depth ranging from approximately $3 / 4$ to $1-1 / 2$ in. The compressibility of soil could significantly alter this depth, but for estimation purposes the depth was assumed to be no greater than $2 \mathrm{in}$. Therefore, the height of the upper box portion was set at 2 in., permitting testing of depths from approximately $1 / 4$ to $2 \mathrm{in.}$ This height, of course, can be altered should particular site conditions dictate. The lower box portion was set at 1-1/4-in. height, including the cutting edge. A 1/8-in. wall thickness was used for both boxes.

7. Figure B3 presents a series of sketches of the specimen container showing the various stages of placement. To minimize specimen 
disturbance, it was decided to use the specimen cutting box as the device container rather than remove the cutting and place a container over the specimen. The box consists of three parts: (a) a lower portion with knife-sharp edges to aid in cutting the soil, (b) an upper portion, and (c) an outer holder to keep the lower and upper portions in alignment. The box is alternately pushed and trimmed into the soil to the desired depth. Once in place, the outer holder can be carefully removed, leaving the two boxes on the specimen with the joint between the lower and upper box portion forming the shear test plane. Base

8. A relatively narrow 1 -in.-thick aluminum plate was used to construct the base with a square hole at one end to fit around the 4by 4-in. lower specimen containers (Figure B1). The shear loader was attached to the other end of the plate. A second 1-in.-thick aluminum yoke was constructed to fit over the upper specimen container. Set screws through the yoke serve to raise the yoke off the baseplate, thus minimizing friction between the surfaces. The shear loader attached by cable to the yoke pulls the upper specimen while the base reacts against the lower specimen container. Guide rails along the edge of the base ensure that no torsional shear deformation or twisting is applied to the specimen.

Shear loader

9. An electric 12-volt boat winch was incorporated into the base as the shear loader. This approach is the simplest for providing a shear loader. (If necessary, the winch can be replaced with a more sophisticated loader custom-built for this device.) Currently, the winch is capable of pulling loads up to $20001 \mathrm{~b}$. Static loading rates can be applied by manually turning the winch via a socket-rachet arrangement. Fast loading rates (approximately 300-600 msec time to peak load that is equivalent to a strain rate of 0.5 to 1.5 per sec, which is compatible with the strain rate under the track) can be applied using the electric feature of the winch. The power is supplied by a 12-volt car battery, which is also used as the instrumentation power supply. 
Instrumentation

10. A 2-in. travel film potentiometer is attached to the base and records relative movement between the upper specimen holder and the base. A strain gage load cell attaching the winch cable to the specimen yoke is used to measure shear load. A compact, two-channel DC instrumentation amplifier is used for signal conditioning. Output is recorded in the form of a shear load versus deflection plot on a commercially available DC-operated X-Y plotter. As previously mentioned, a simple car battery is the main power supply. All initial testing was done by recording the data on a time base light beam strip chart. This recording procedure was later dropped since the loading times remained fairly constant on the soils tested. A time base can be added at a later date through the use of a frequency oscillatory and an $\mathrm{X}-\mathrm{Y}-\mathrm{Z}$ recorder. Normal load

11. A series of steel weights, the largest weighing $57 \mathrm{lb}$ and the smallest weighing $8-1 / 21 \mathrm{~b}$, was fabricated for use with the device. Guide holes and studs permit stacking and centering of the weights on the specimen top surface. Although a variety of load combinations are possible, most tests have been conducted using weights totalling approximately $8.6,36.6,65.7$, and $122.7 \mathrm{lb}$ (i.e., normal stress levels of $0.54,2.29,4.11$, and $7.67 \mathrm{psi}$ ).

\section{Measurement of Soil Parameters}

12. A series of two or more tests is required at a site to define the necessary soil parameters. A typical testing program may call for the conduct of four fast and four slow tests at normal stresses of $0.54,2.29,4.11$, and $7.67 \mathrm{psi}$. For each test an X-Y data record of shear load versus deflection is obtained. In addition, measurements of soil density and water content are made on each test specimen (generally on the posttest specimen contained in the upper and lower specimen holders). 
13. For each test a plot of shear stress versus deflection is obtained. The initial slope of the plot defines $G$, the peak stress defines the maximum shear stress, and the deflection at peak stress divided by time to peak stress defines the deflection rate. A table listing of each test is used to summarize the data and contains the specimen number, wet density, water content, dry density, normal load/stress, maximum shear load/stress, initial G, deflection at peak stress, and deflection rate. Figure B4 presents the test results obtained from the series of field tests conducted at a given site.

14. The analysis plots are shown graphically in Figure B5. A summary plot of shear stress versus shear deformation is made to obtain either static or dynamic failure envelopes. From the static failure envelope, the values of $\mathrm{A}, \mathrm{M}$, and $\mathrm{N}$ are obtained. The value of $C_{d}$ and $\Lambda$ are obtained from the dynamic failure envelope as shown in the plot of cohesion versus rate of deformation. The value of $G$ is the initial slope of shear stress-shear deformation curve (Figure B4).

\section{Conclusions}

15. A new agility model for track-laying vehicles was developed that required soil parameter input not commonly obtained during mobility studies. It was realized that field sampling and laboratory testing would not always be possible because of the nature of very near-surface soil deposits. An approach was taken to use a conventionally accepted test to define the parameters. A field-operable direct shear device and necessary instrumentation were built at the WES. The equipment is fairly compact (can easily fit into a car or truck), operates off of a car battery, is relatively simple to use, saves operation time compared with comparable laboratory tests, and is capable of directly accessing the soil parameters.

16. The device has been used to conduct some 123 tests at several different locations. Some of the near-surface soils encountered at these locations were very friable and impossible to sample and test in 
the laboratory by conventional means without excessive disturbance. However, the direct shear device performed quite we11, only requiring extra care by the test operator not to disturb the specimen during placement of the device base over the sample box containing the soil specimen. The time required to perform a test was approximately onehalf hour. This time is especially reasonable when compared with the time it takes to prepare and test a comparable sample in the laboratory, excluding the time spent obtaining the sample in the field. 


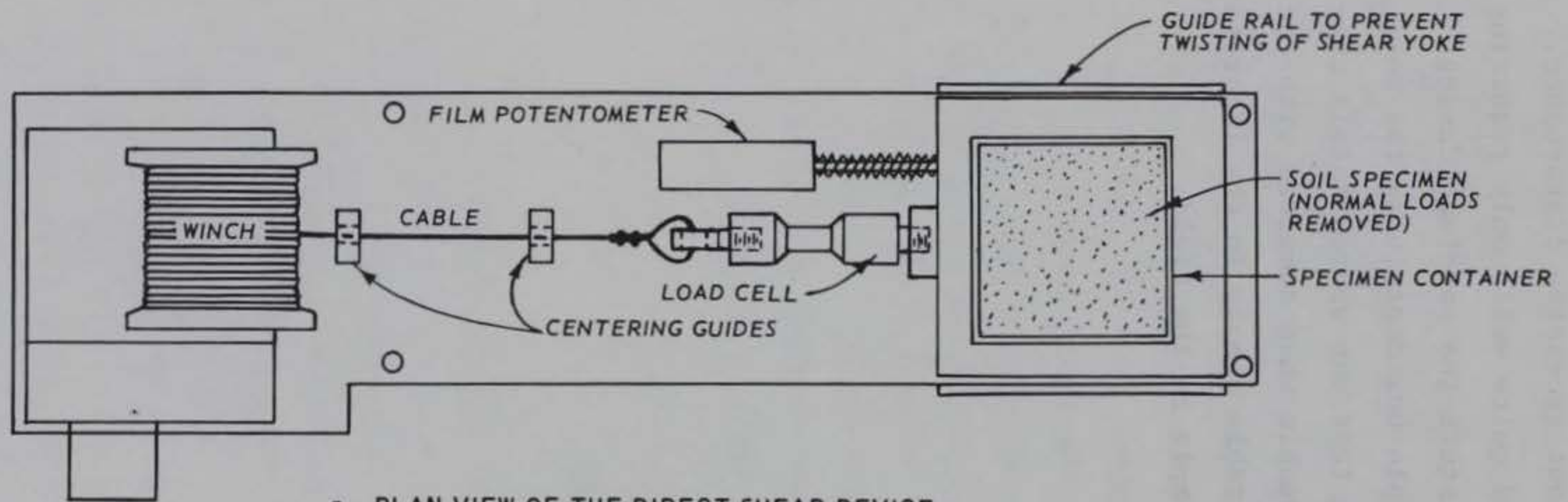

a. PLAN VIEW OF THE DIRECT SHEAR DEVICE

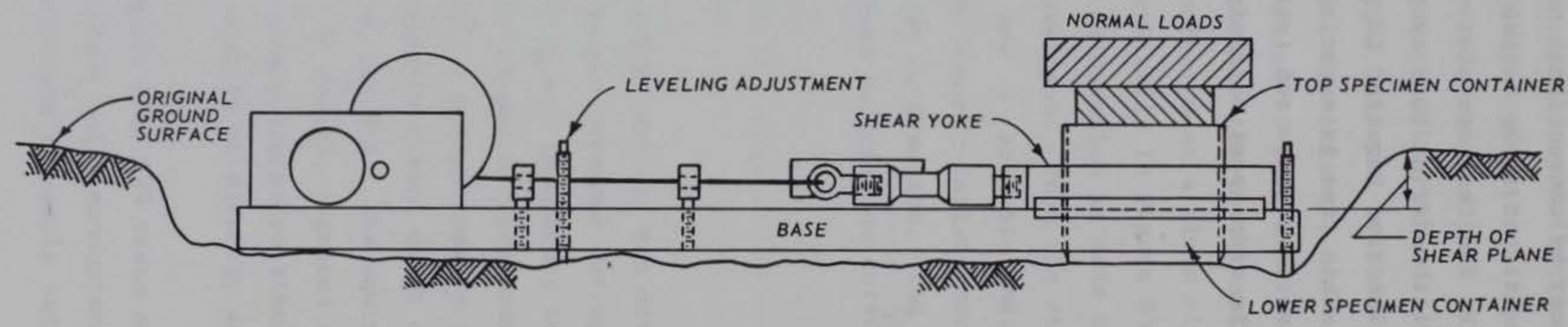

b. PROFILE VIEW OF THE DIRECT SHEAR DEVICE IN PLACE

Figure B1. P1an and profile views of the direct shear device 


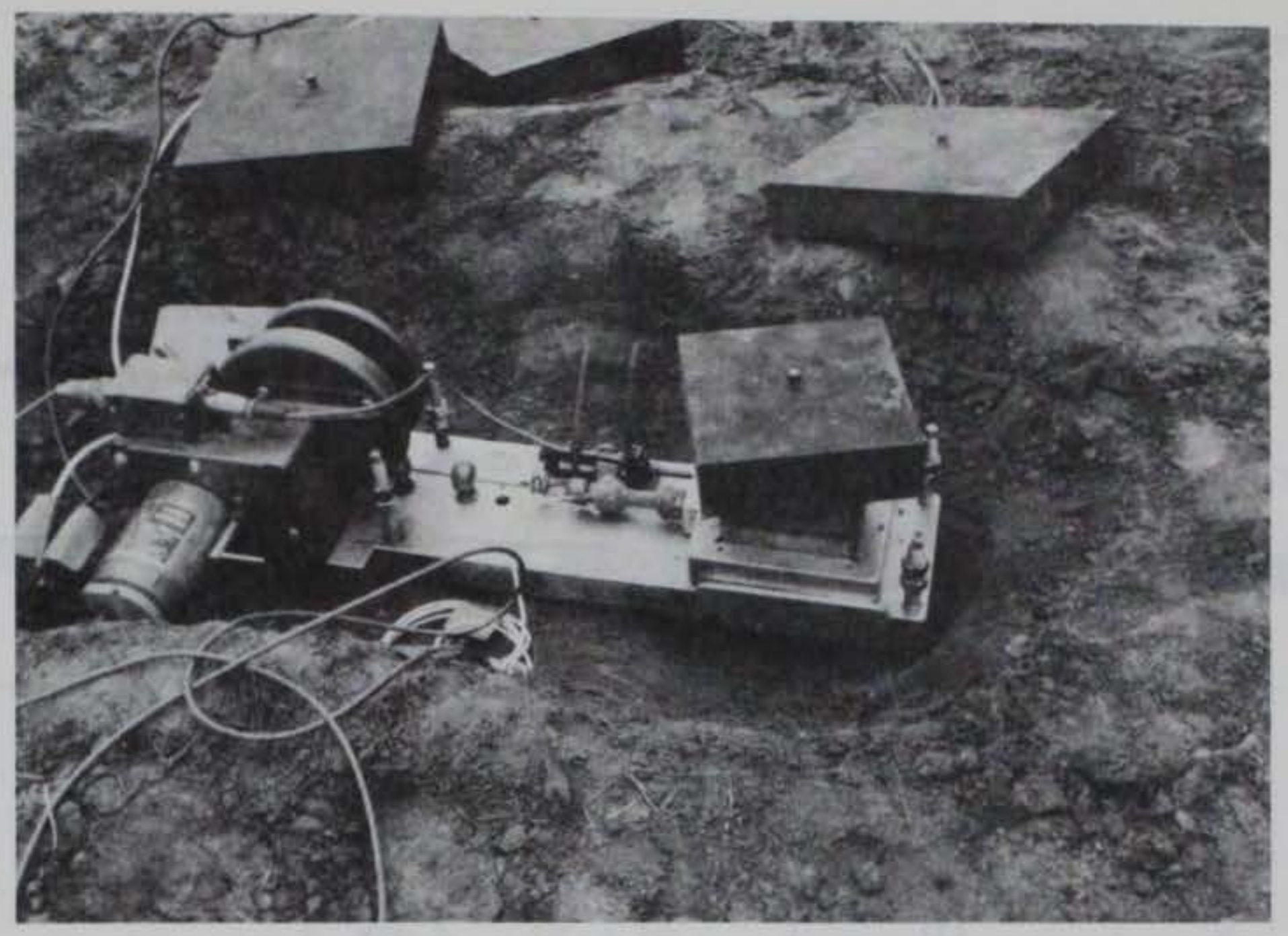

a. Direct shear device assembled for test

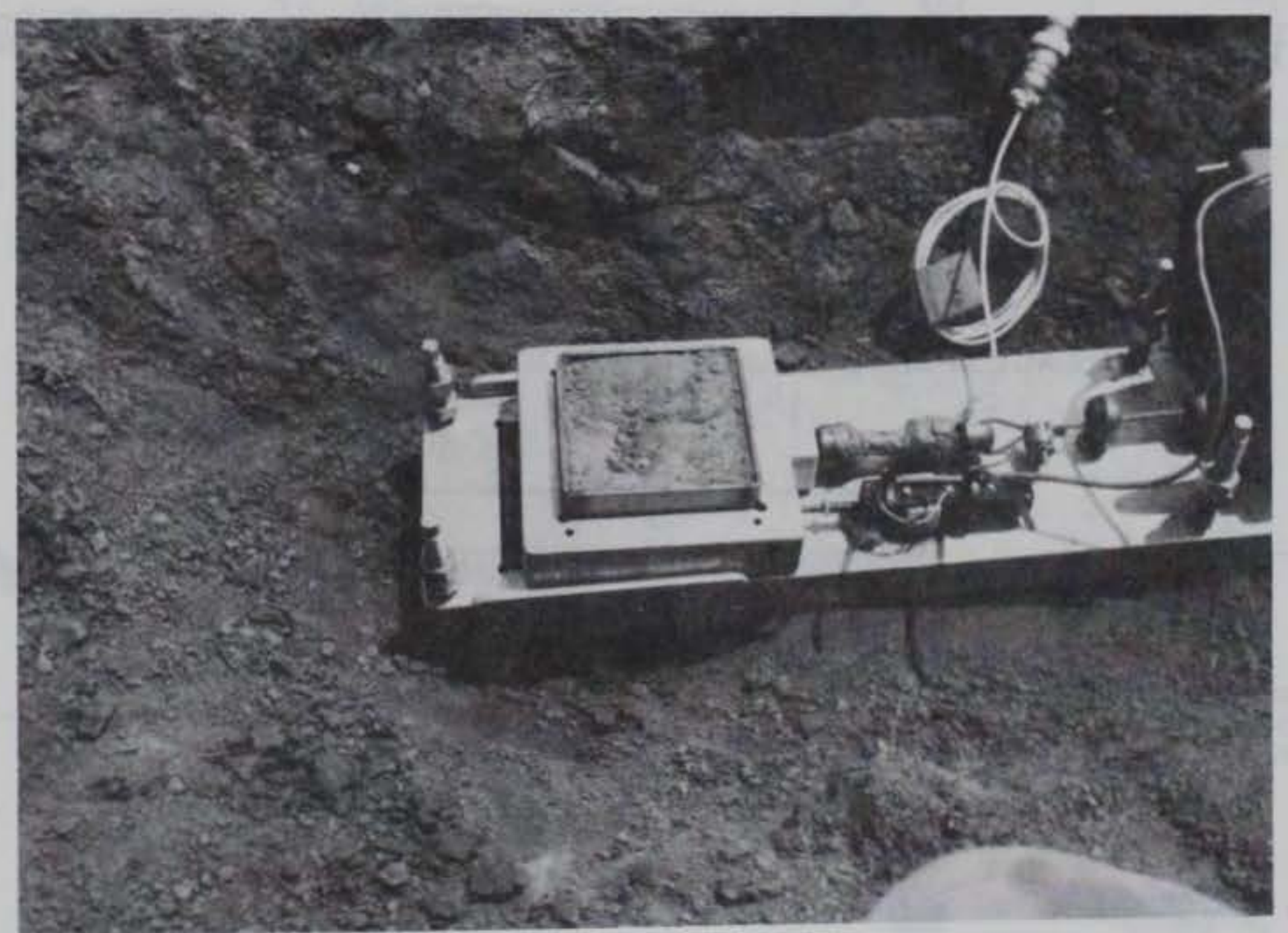

b. Specimen immediately following test with normal loads removed

Figure B2. Photographs of the direct shear device taken during actual field testing 


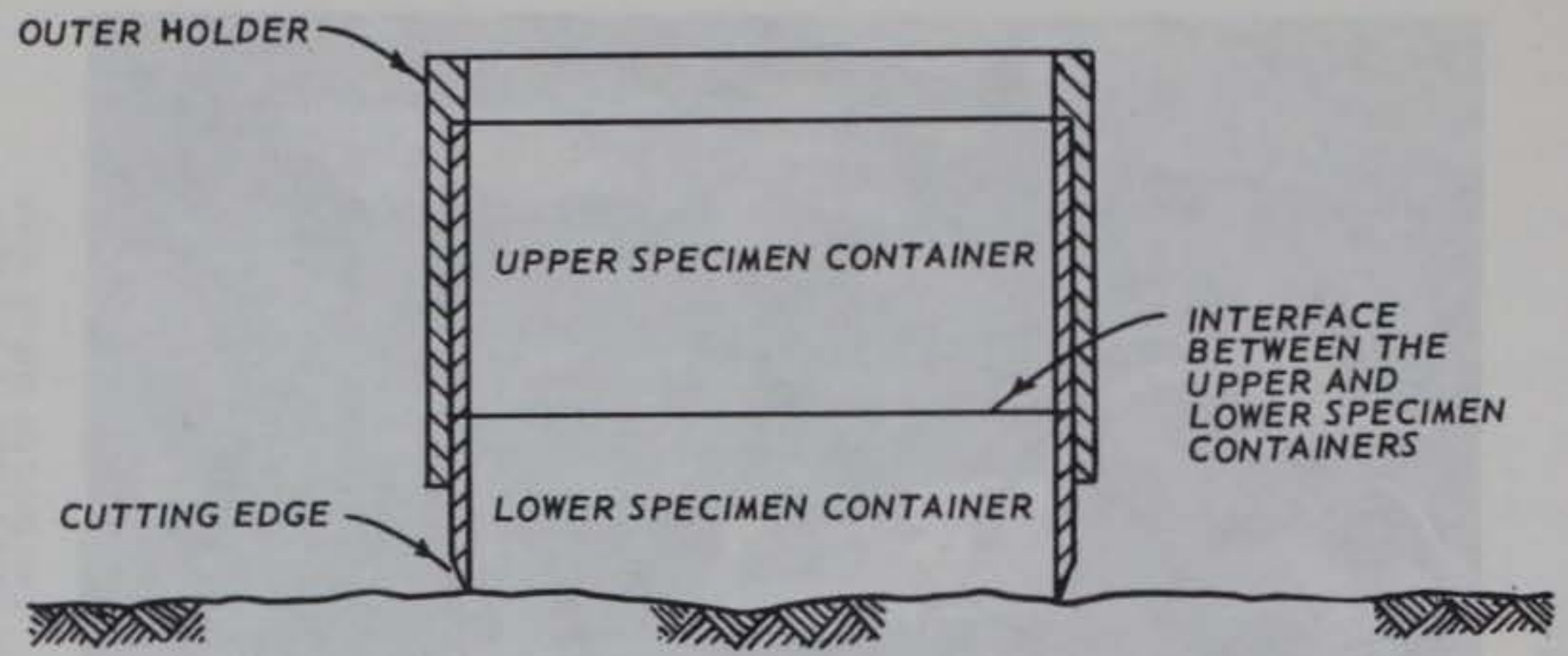

a. CONTAINER WITH OUTER HOLDER ON SOIL SURFACE

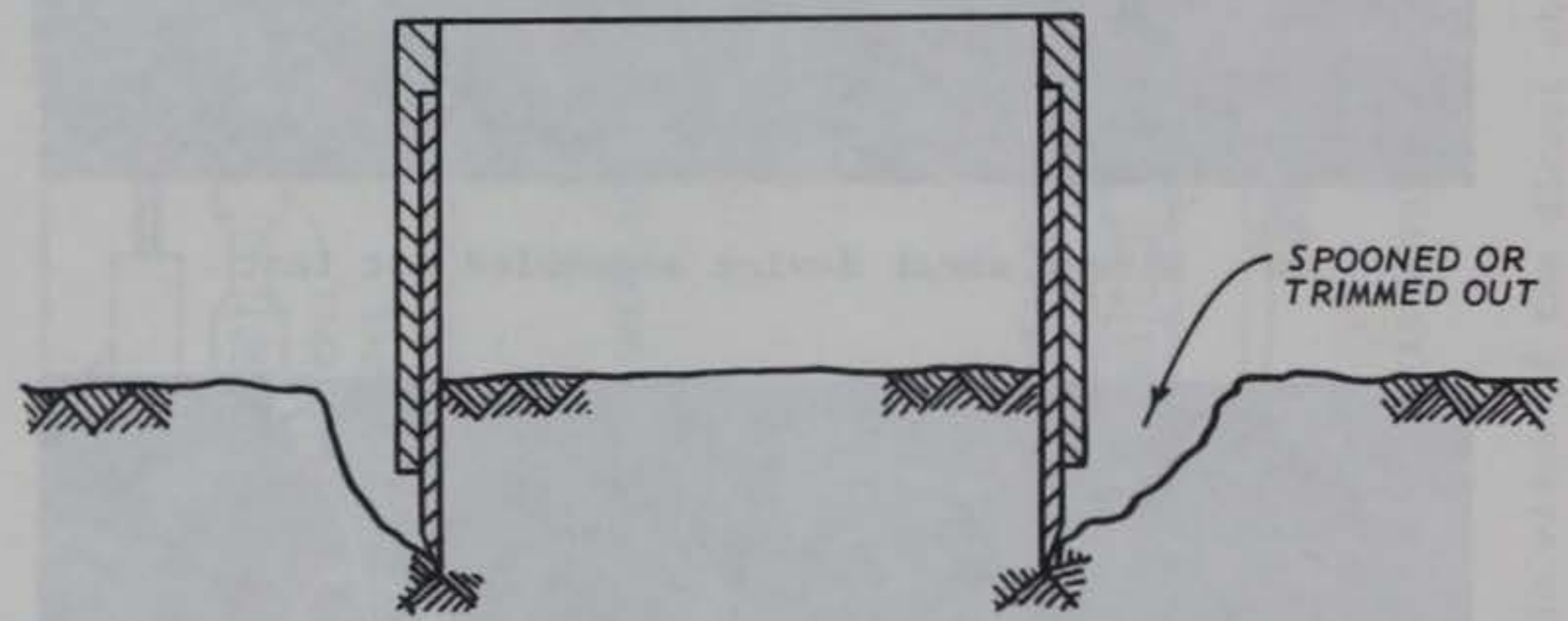

b. CONTAINER DURING PLACEMENT - ALTERNATELY PUSHED AND EXCESS MATERIAL SPOONED (OR TRIMMED) OUT

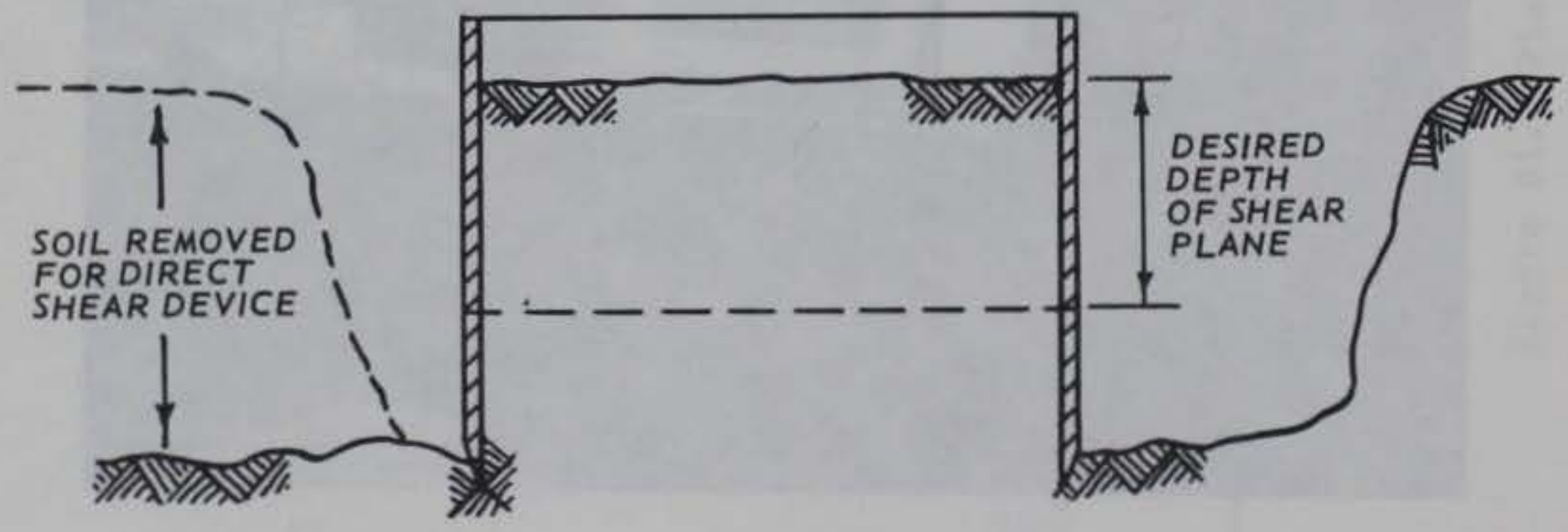

c. CONTAINER AT DESIRED DEPTH - OUTER HOLDER REMOVED AND READY FOR PLACEMENT OF DIRECT SHEAR DEVICE

Figure B3. Cross sections through the specimen container showing various stages of placement 

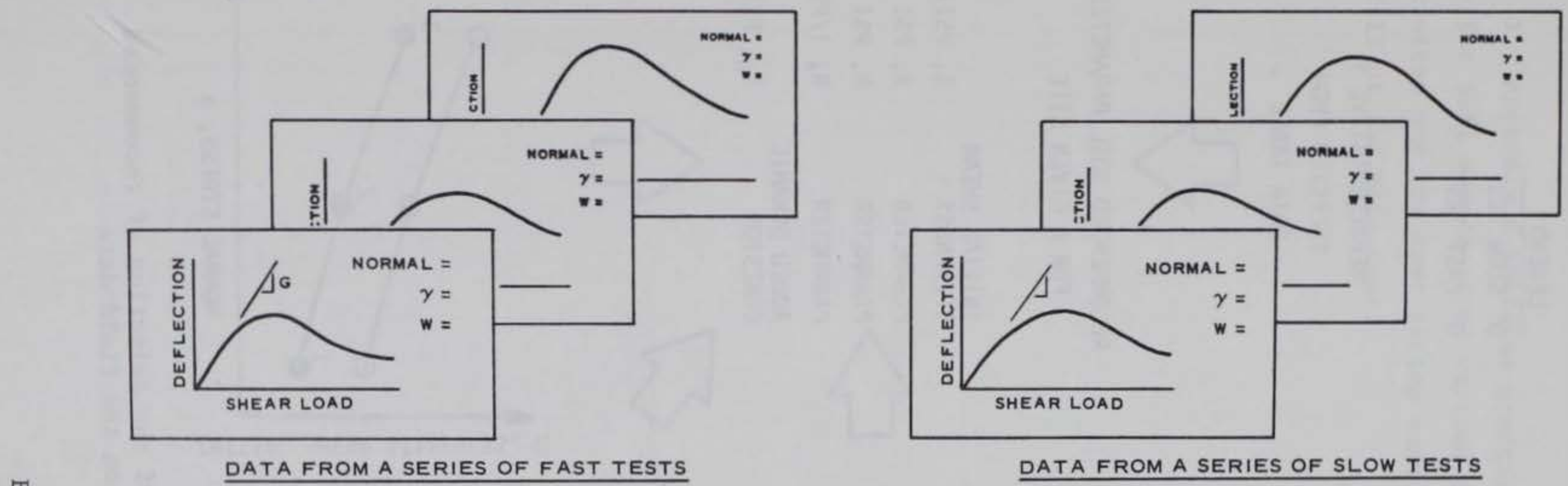

罟

DATA FROM A SERIES OF FAST TESTS

DATA FROM A SERIES OF SLOW TESTS

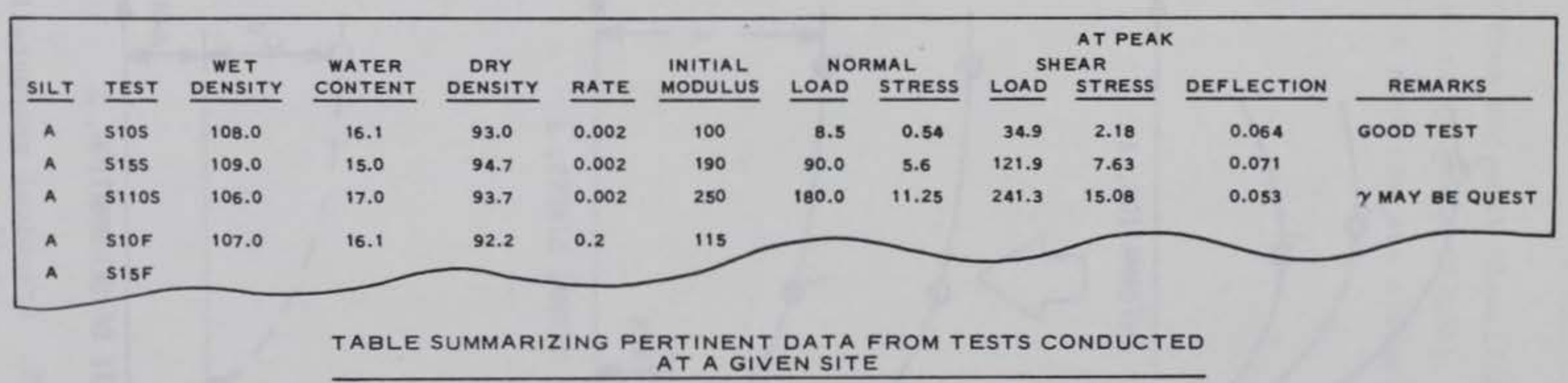

Figure B4. Presentation of test results obtained from the direct shear device 

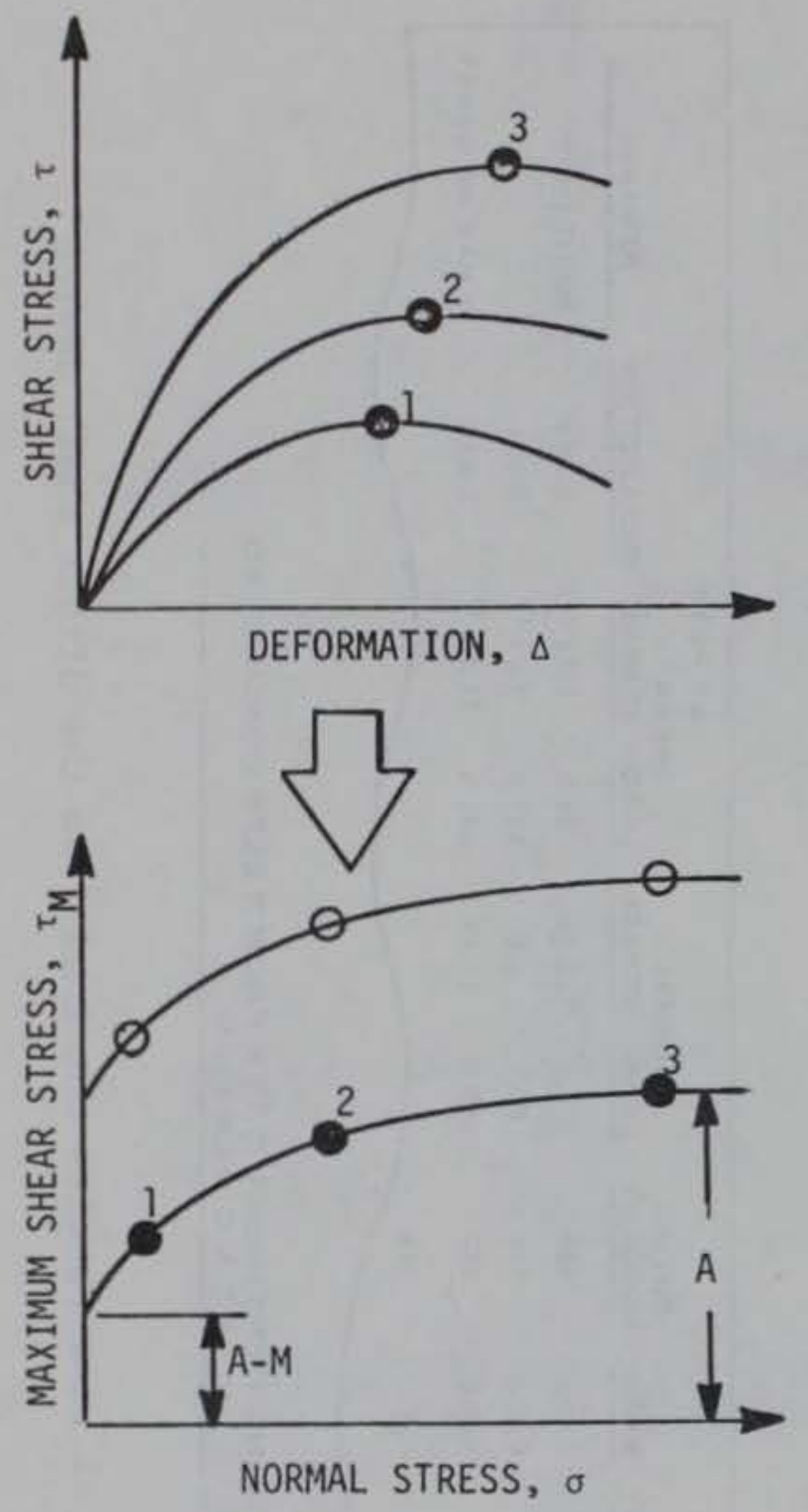

\section{LEGEND \\ 0 SLOW TEST \\ 0 FAST TEST}

REPRESENTATIVE $\gamma$ SELECTED FROM DATA TABLE

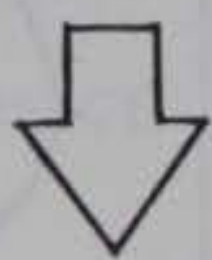

RECOMMENDED SOIL PARAMETER FOR A GIVEN SITE

INITIAL SHEAR STIFFNESS G, PSI/IN.

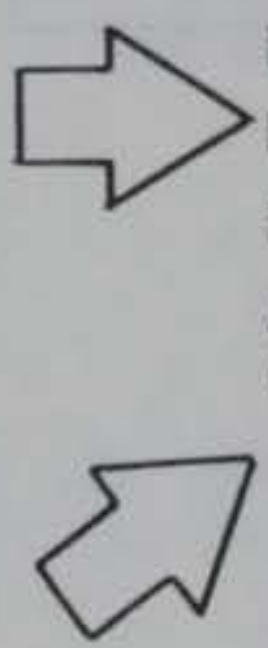

PARAMETER

PARAMETER

PARAMETER

ADDED DYNAMIC COHESION

A, PSI

M, PSI

$N, 1 / P S I$

$c_{d}$, PSI
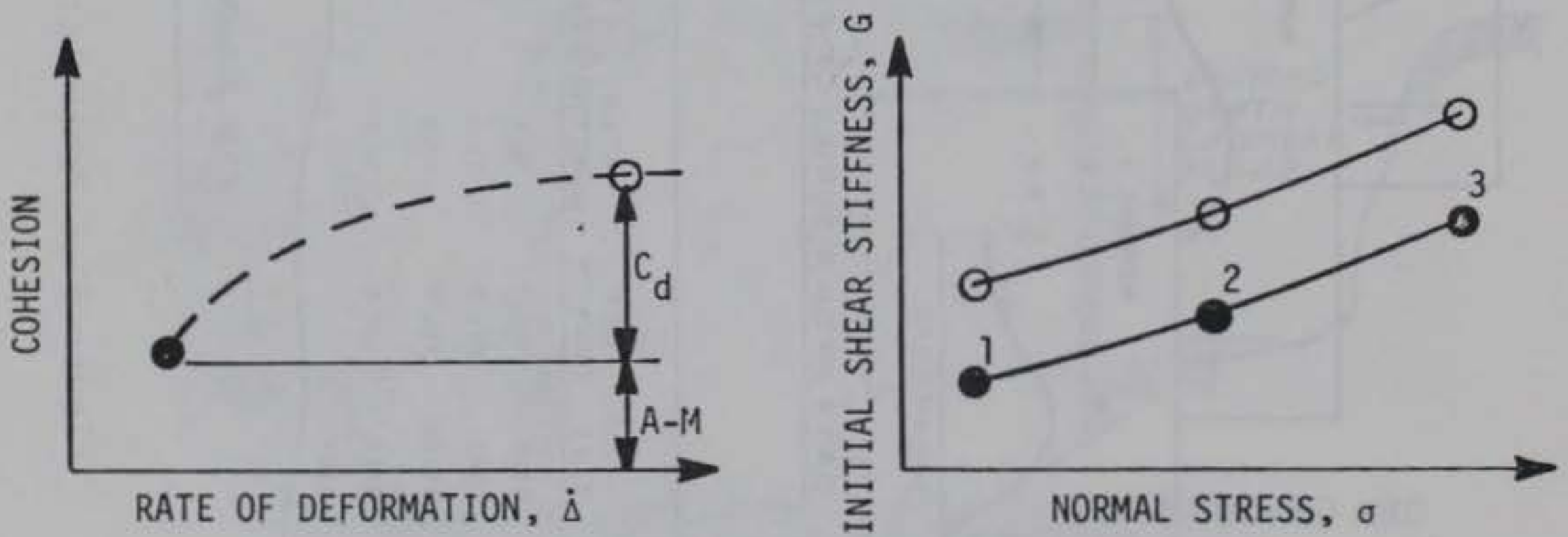

Figure B5. Graphical presentation of the selection of recommended soil parameters based on the field data 
APPENDIX C: SOIL CLASSIFICATION TEST DATA

1. Appendix $\mathrm{C}$ contains gradation curves and soil classification data for soil samples from various test sites (Figures C1-C24). These test sites and the test series conducted at each one are discussed in Part II of the main text. 


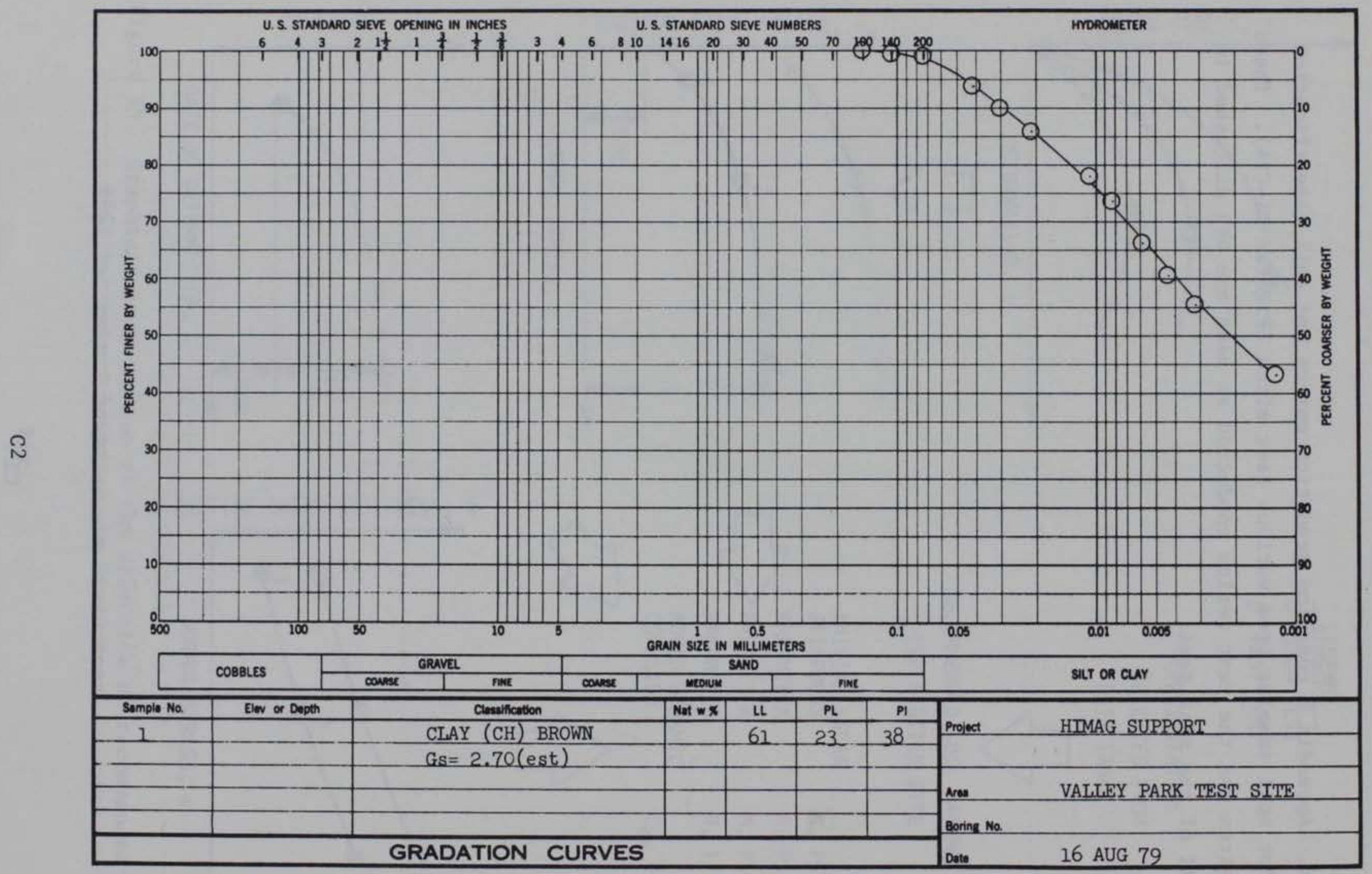

Figure C1. Gradation curve for site 7A, test series 98-102, sample 1 


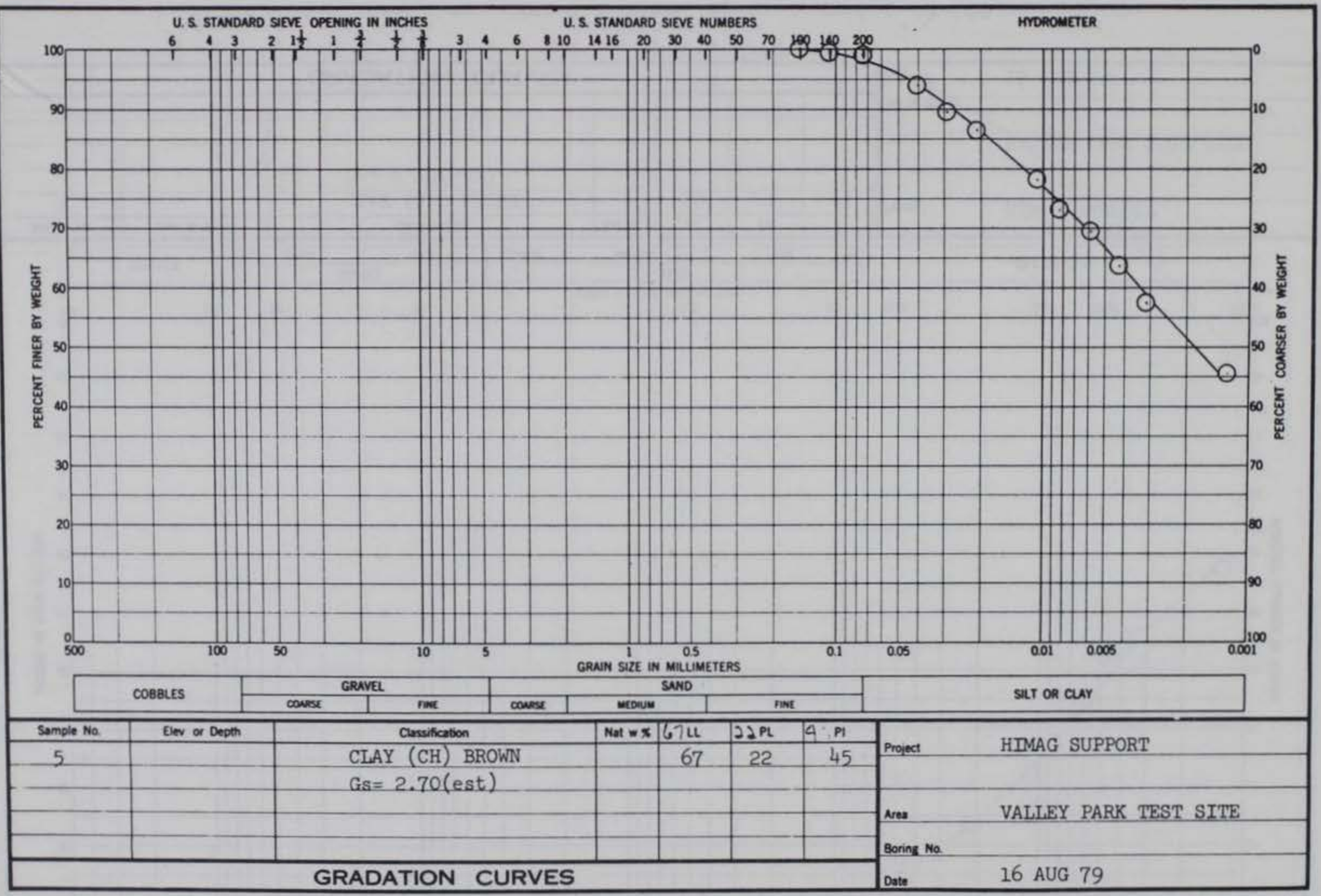

Figure C2. Gradation curve for site 7A, test series 98-102, sample 5 


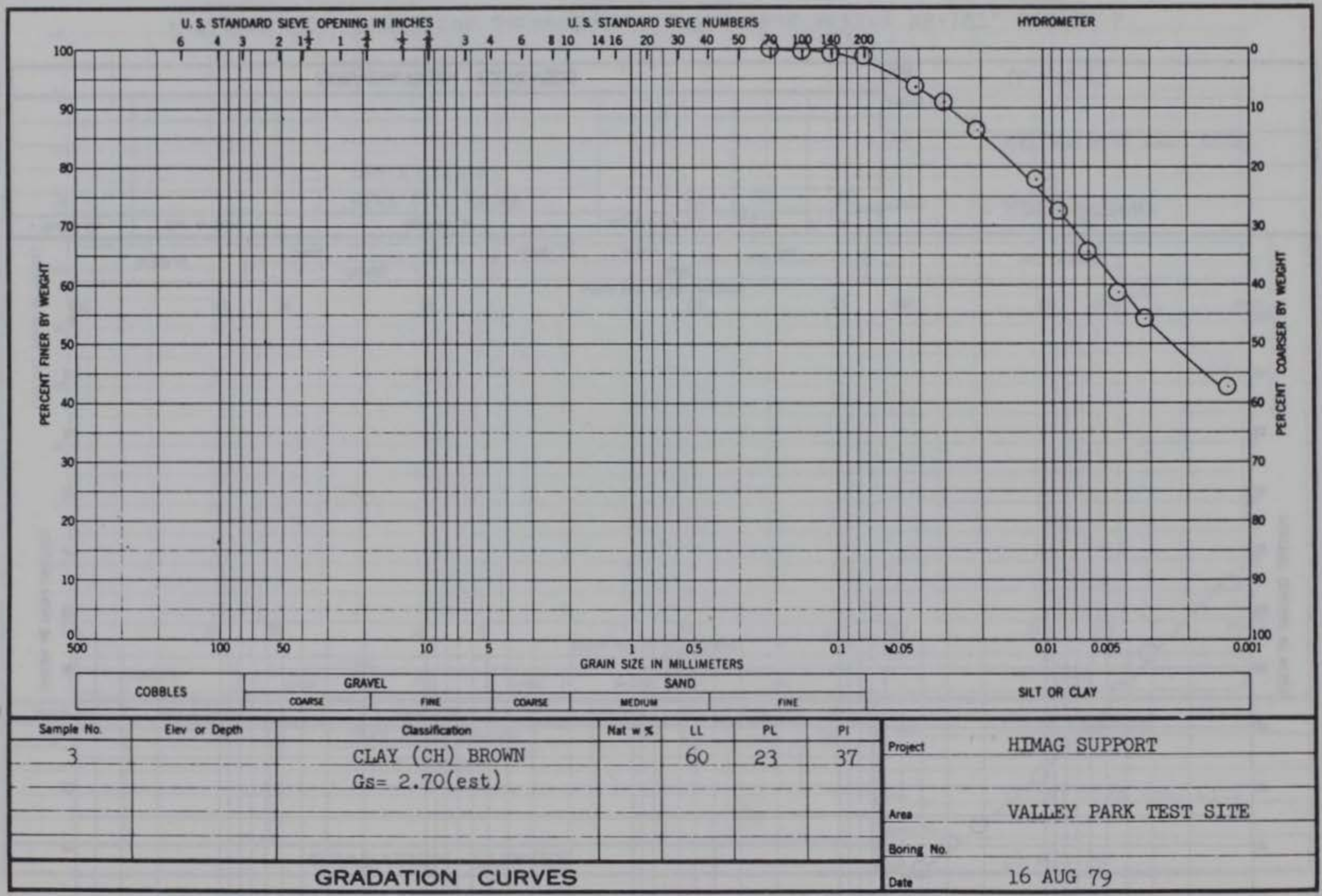

Figure C3. Gradation curve for site 7A, test series 98-102, sample 3 


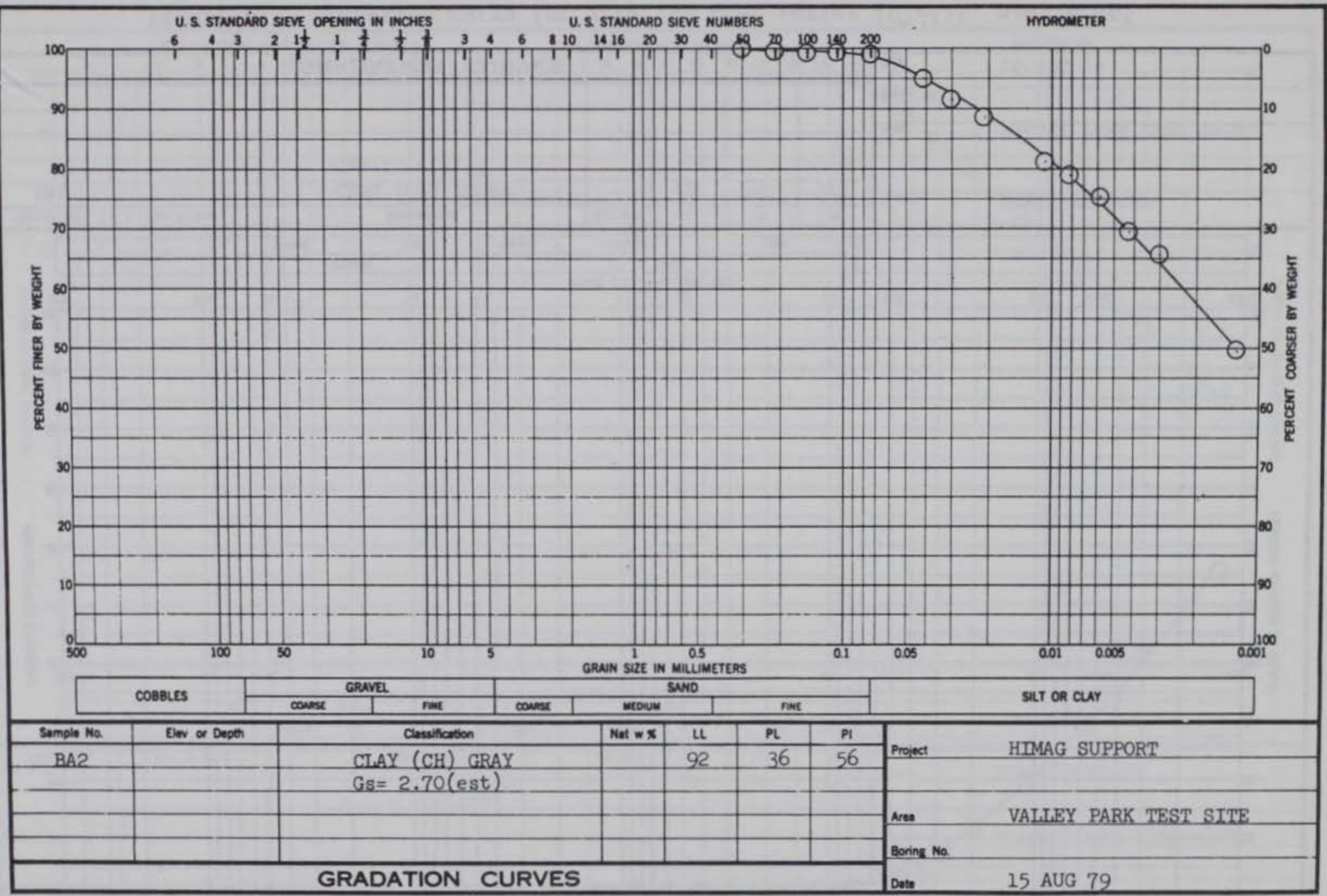

Figure C4. Gradation curve for site 7B, test series 107-111, sample BA2 


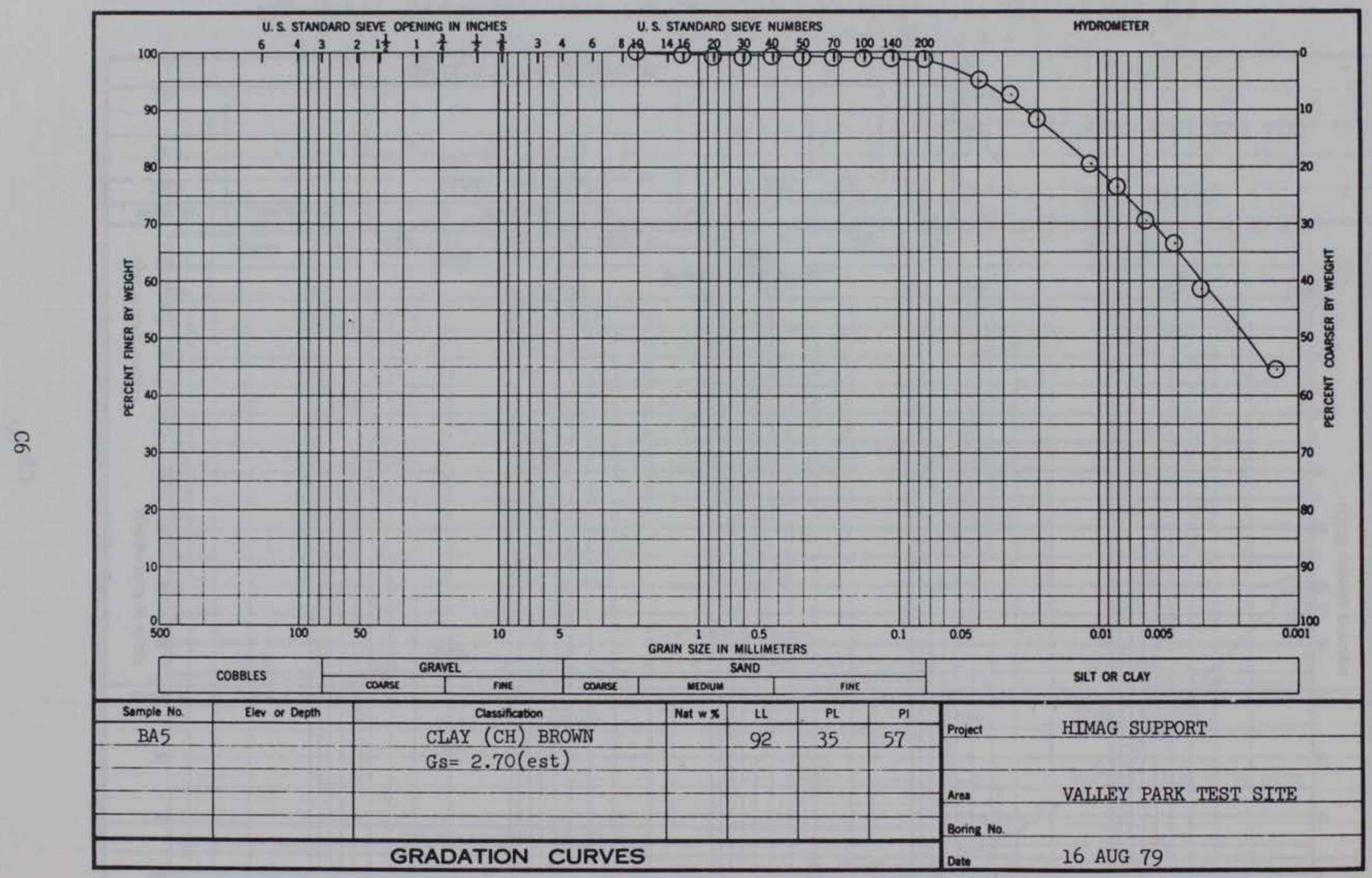

Figure C5. Gradation curve for site 7B, test series 107-111, sample BA5 


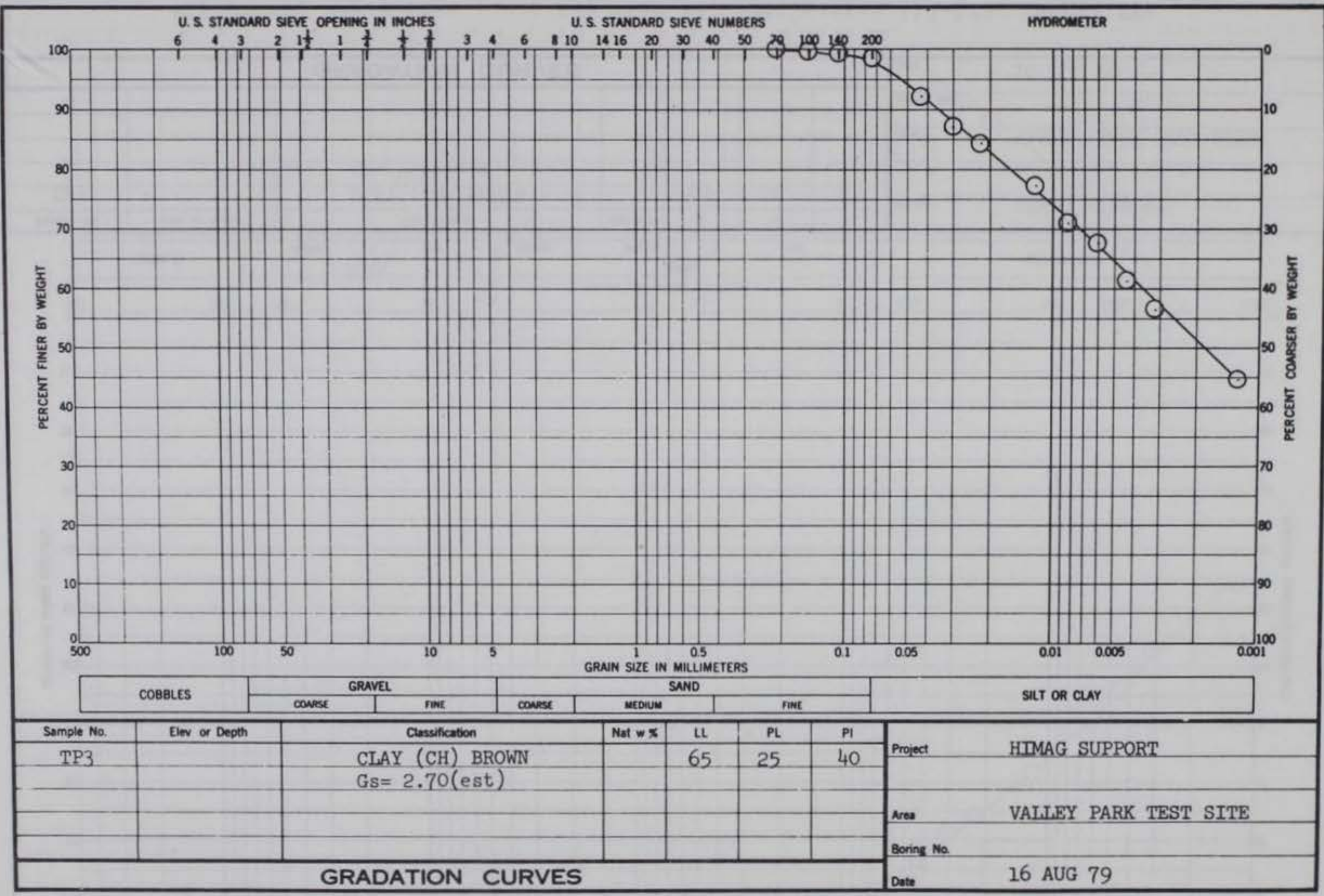

Figure C6. Gradation curve for site 7A, test series 117-122, sample TP3 


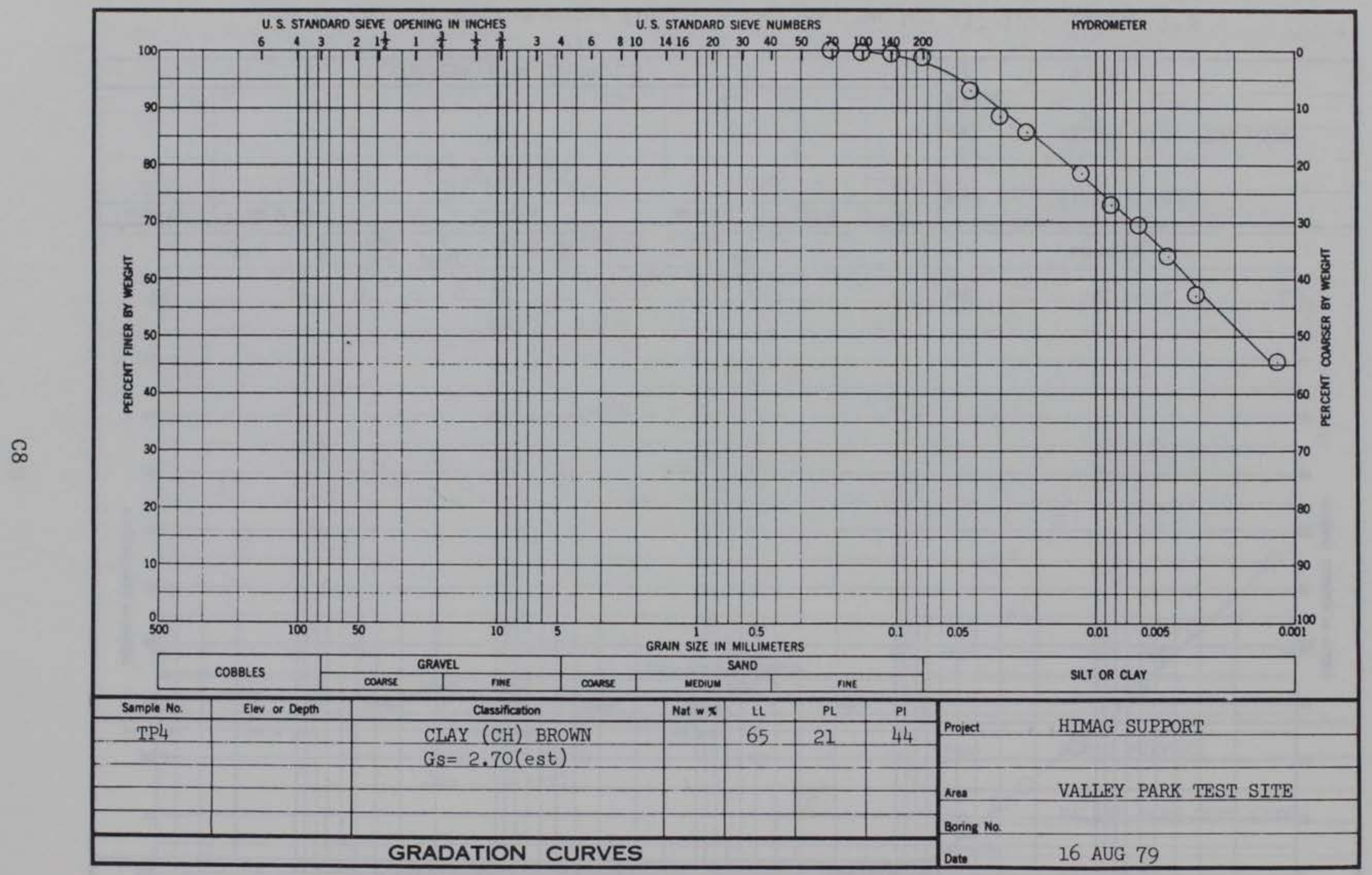

Figure C7. Gradation curve for site 7A, test series 117-122, sample TP4 


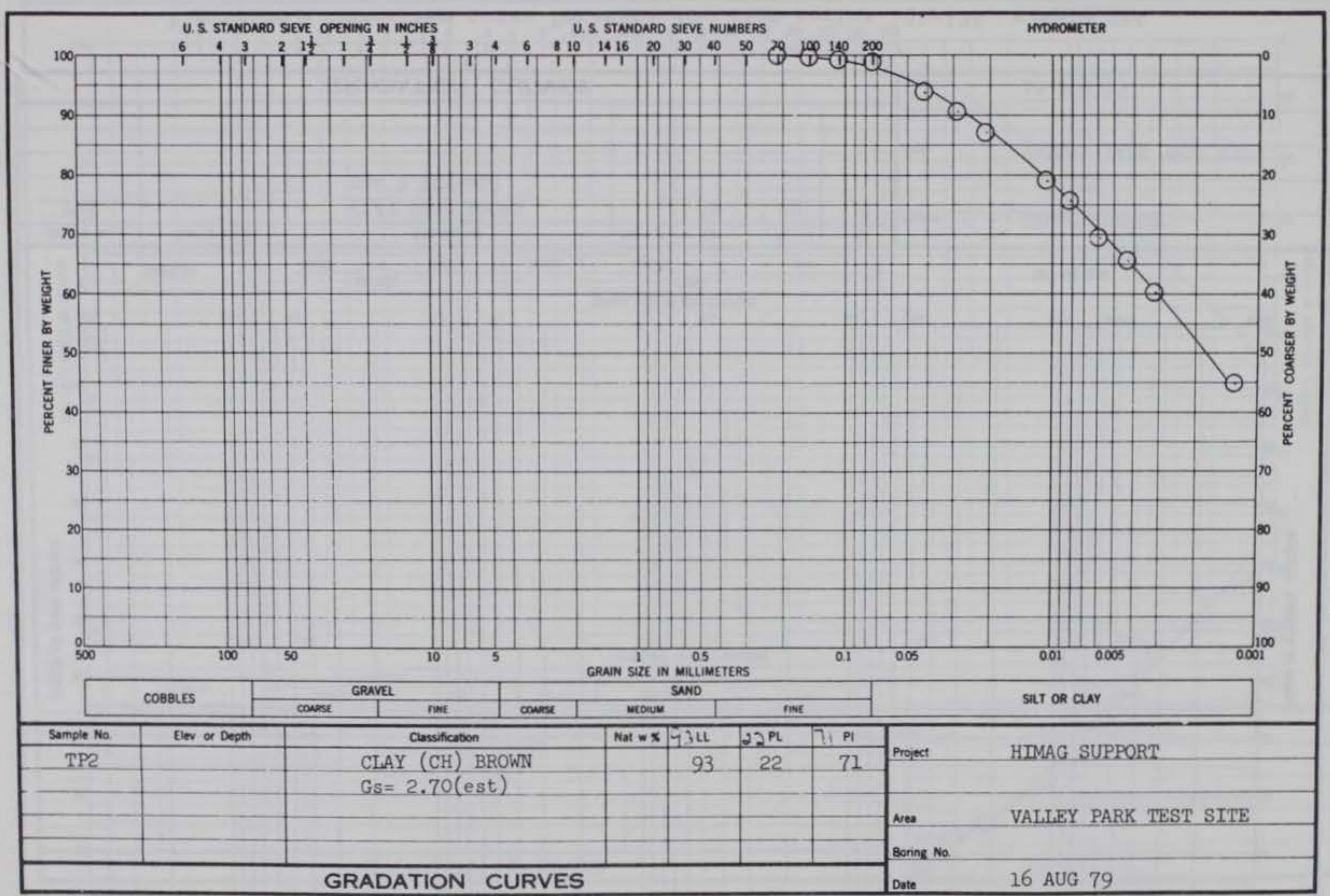

Figure C8. Gradation curve for site 7A, test series 117-122, sample TP2 


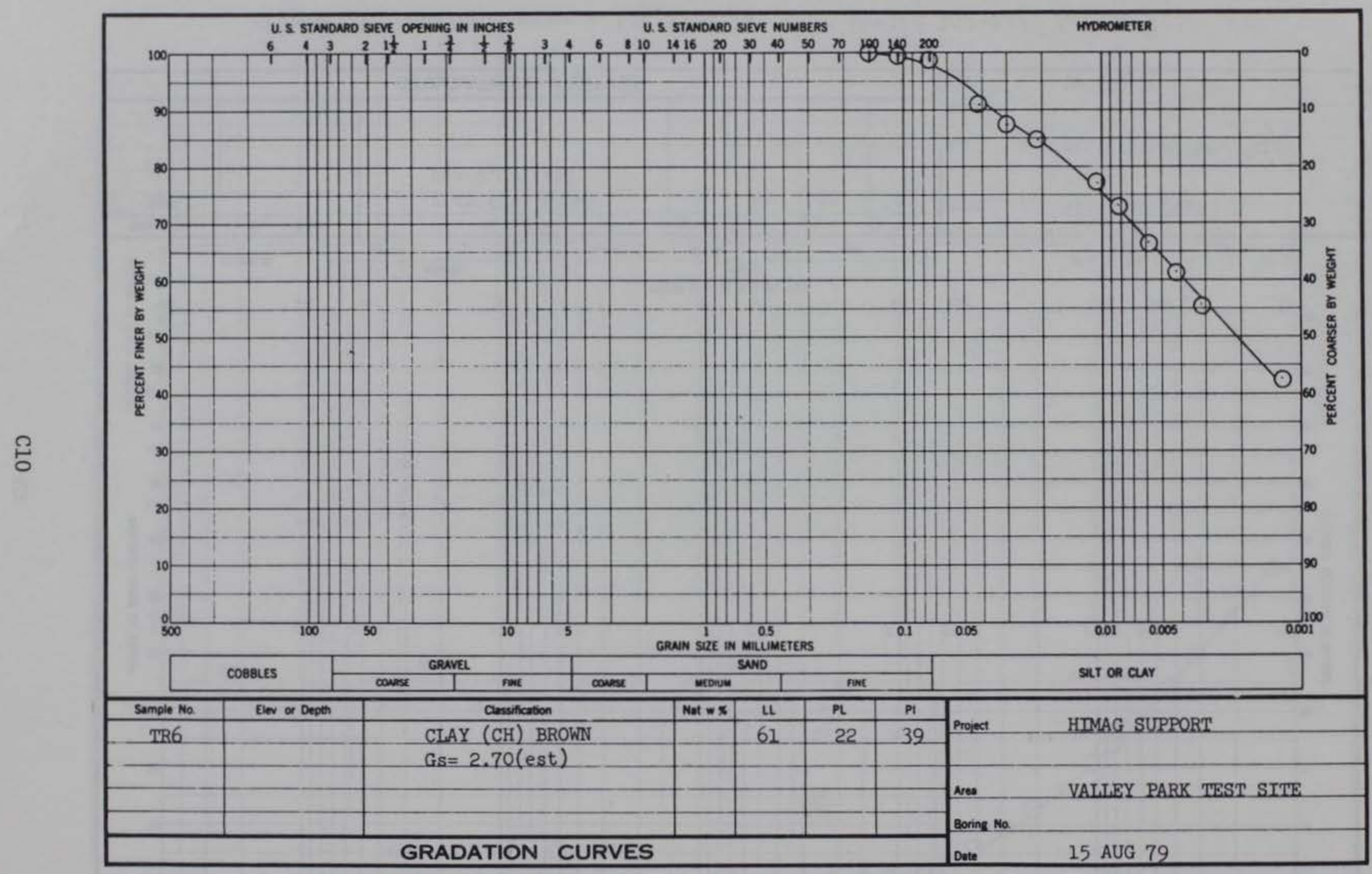

Figure C9. Gradation curve for site 7A, test series 131-134, sample TR6 


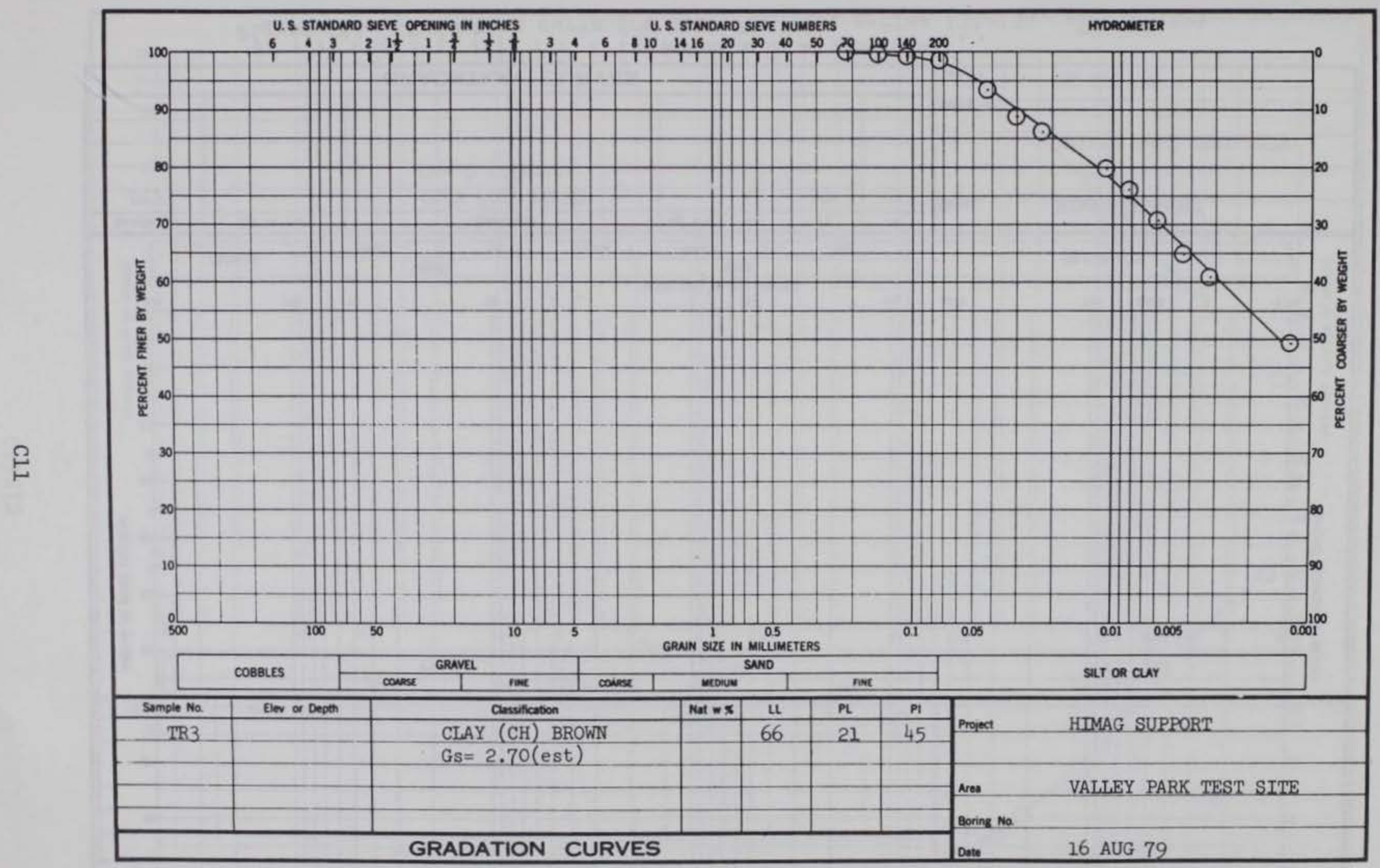

Figure C10. Gradation curve for site 7A, test series 131-134, sample TR3 


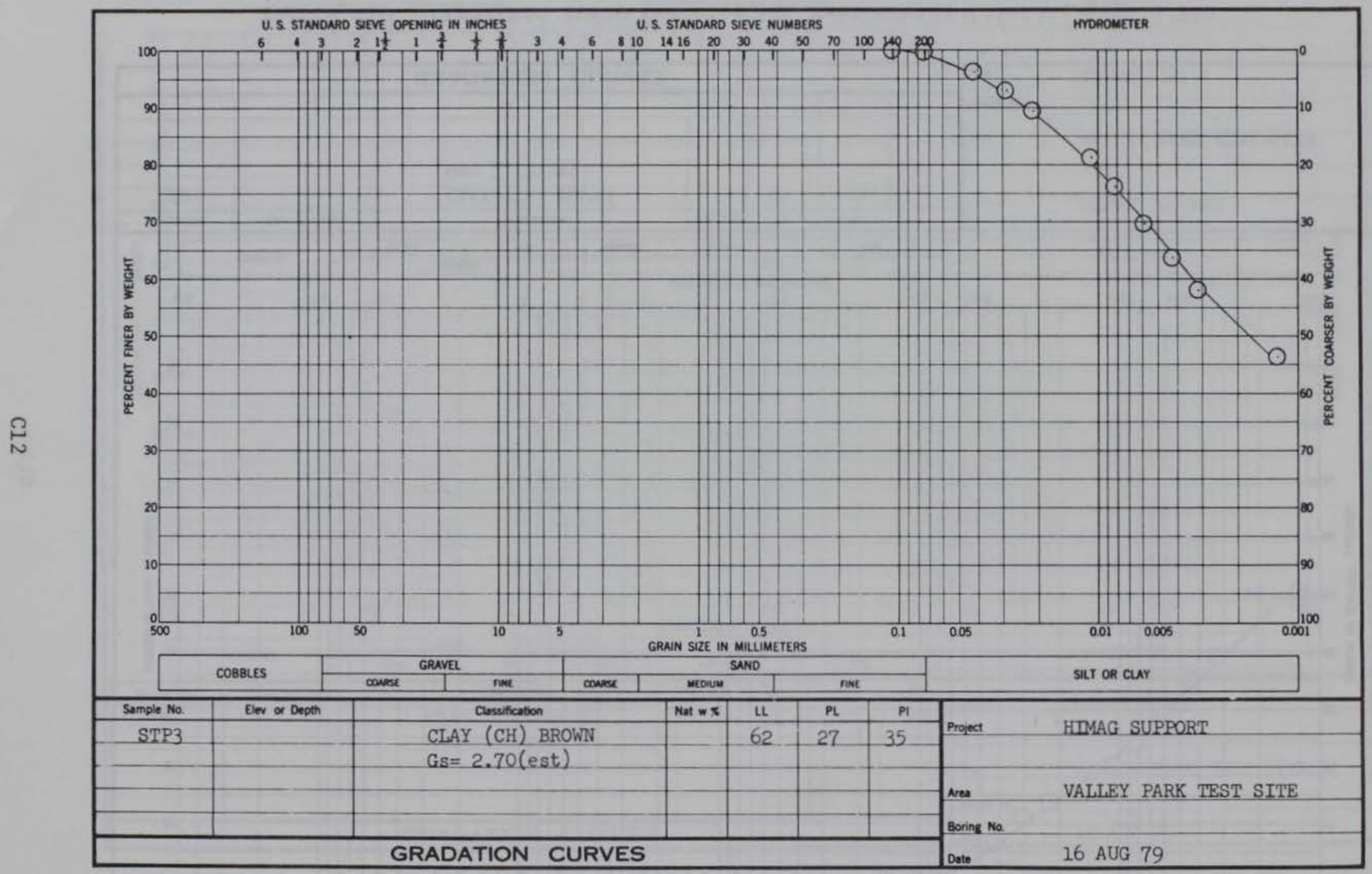

Figure C11. Gradation curve for site 7A, test series 131-134, sample STP3 


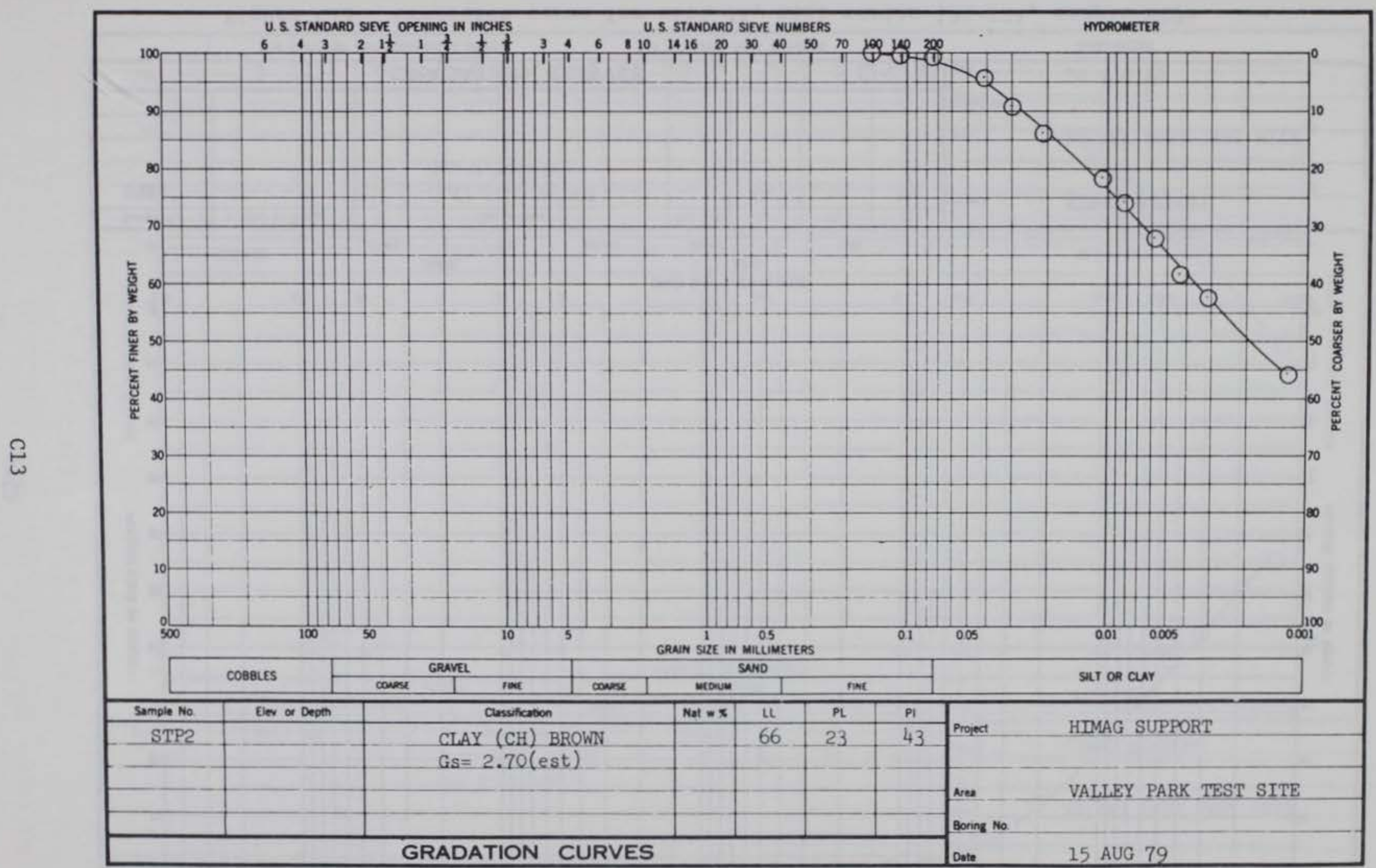

Figure C12. Gradation curve for site 7A, test series 131-134, sample STP2 


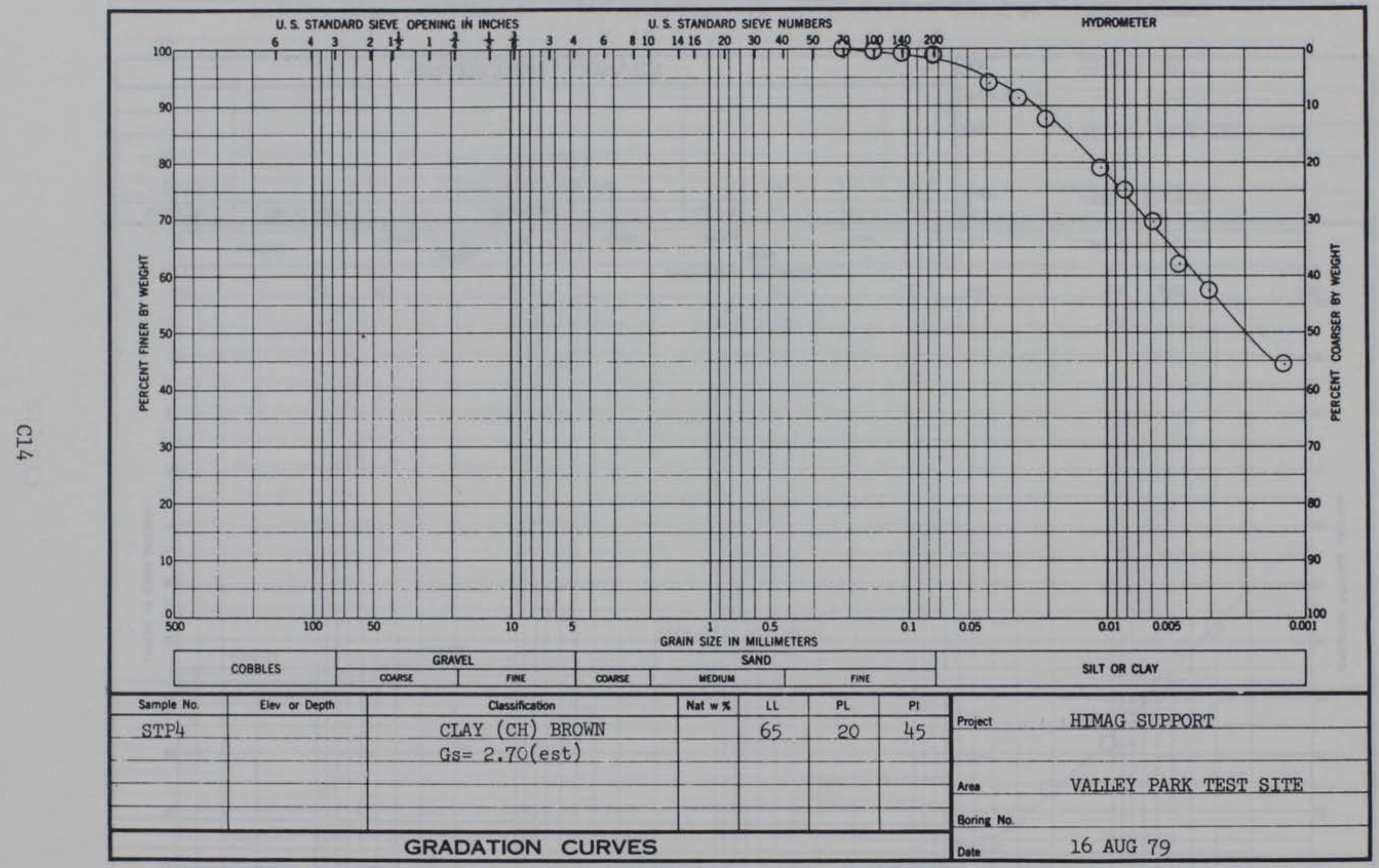

Figure C13. Gradation curve for site 7A, test series 131-134, sample STP4 


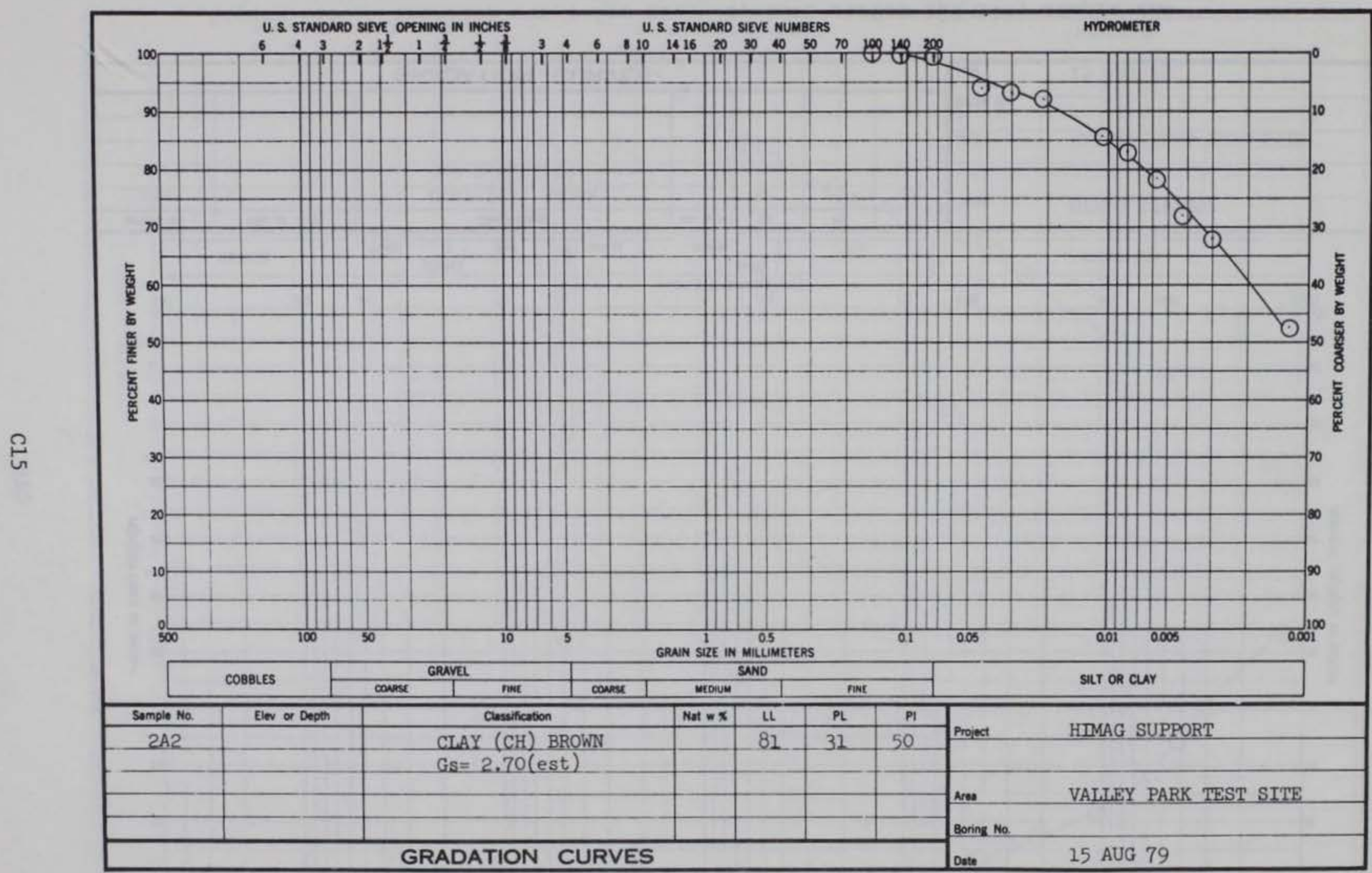

Figure C14. Gradation curve for site 7B, test series 139-144, sample 2A2 


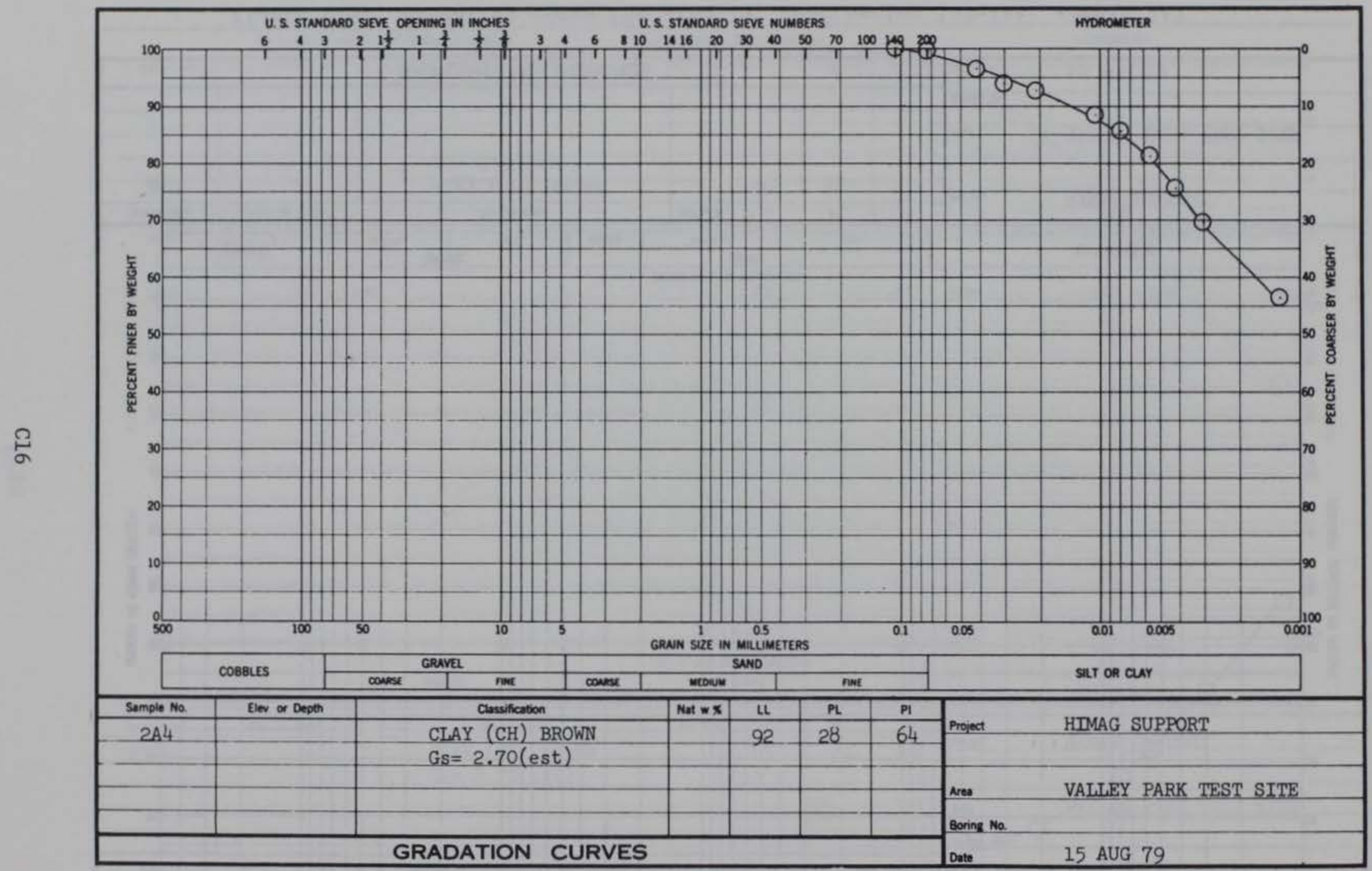

Figure C15. Gradation curve for site 7B, test series 139-144, sample 2 A4 


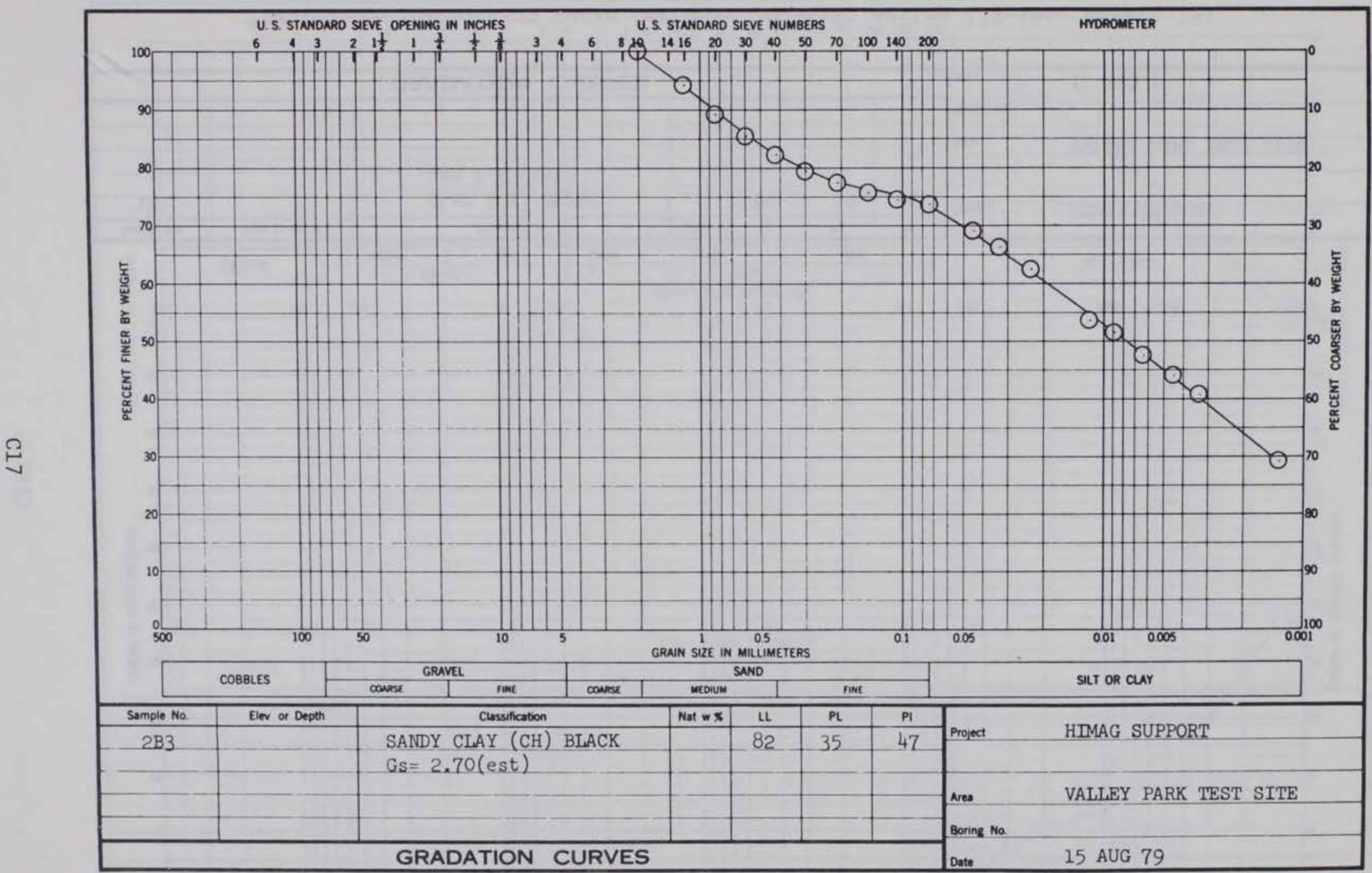

Figure C16. Gradation curve for site 7B, test series 139-144, sample 2B3 


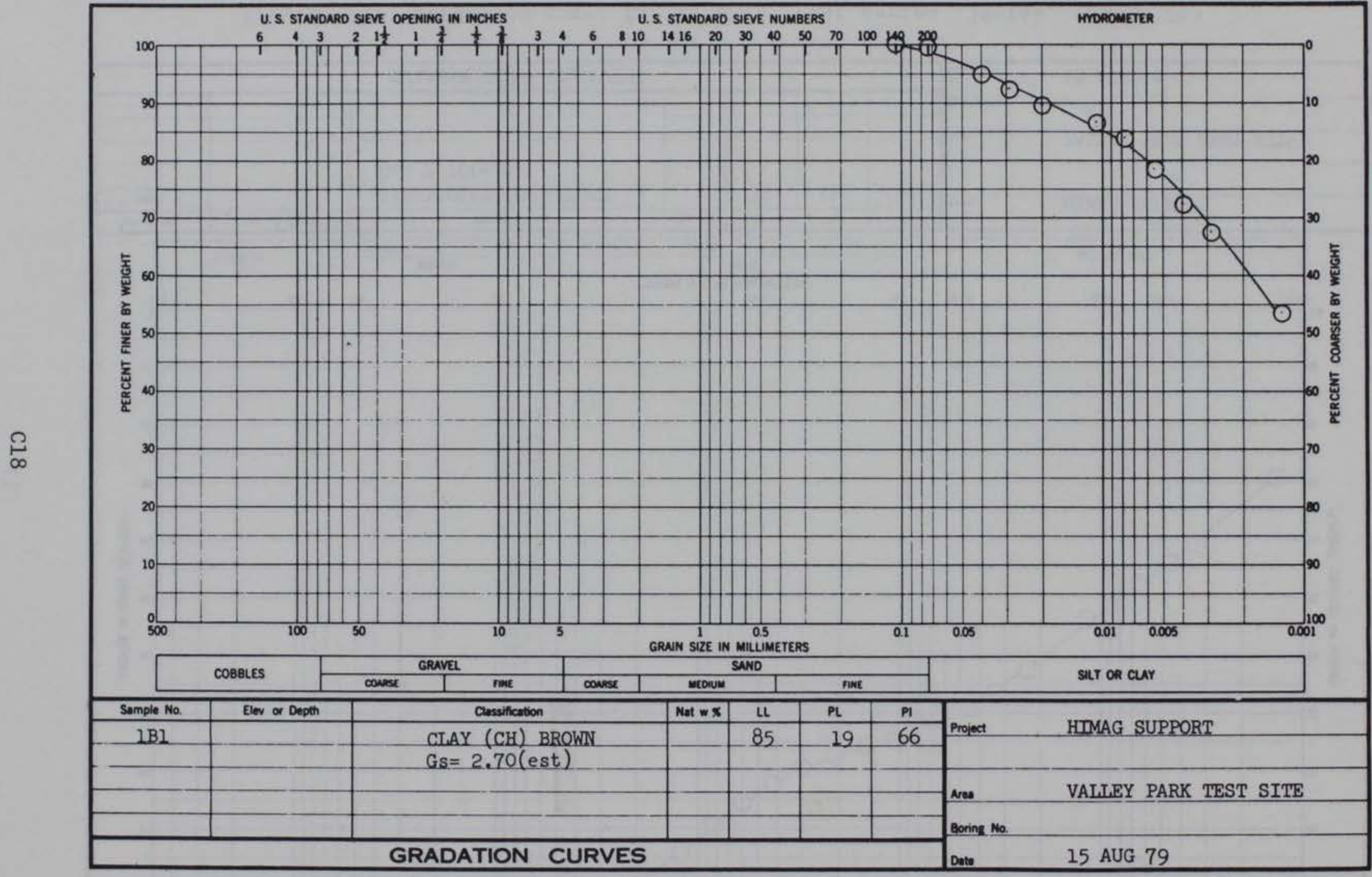

Figure C17. Gradation curve for site 7B, test series 139-144, sample 1 B1 


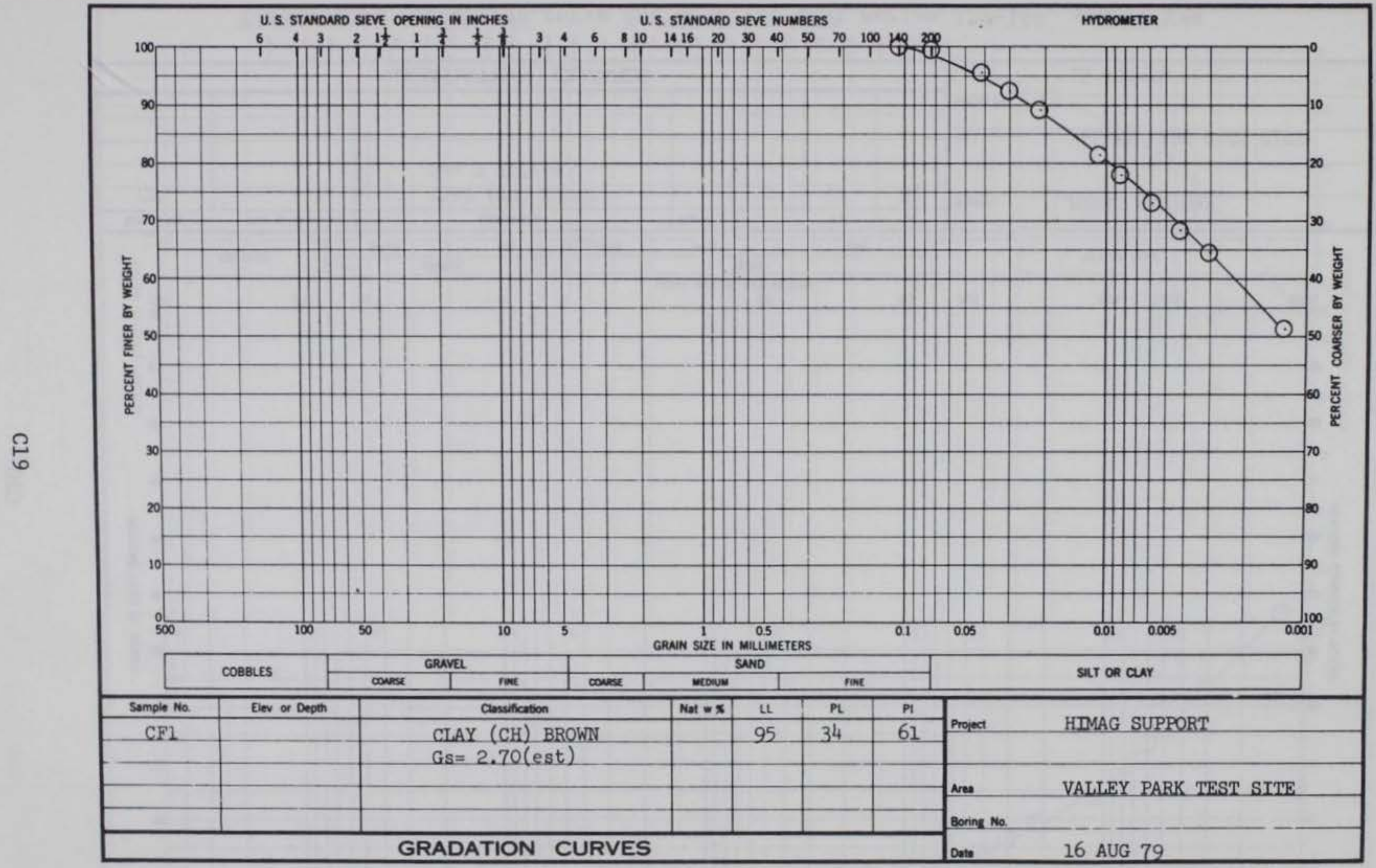

Figure C18. Gradation curve for site 7B, test series 139-144, sample CF1 


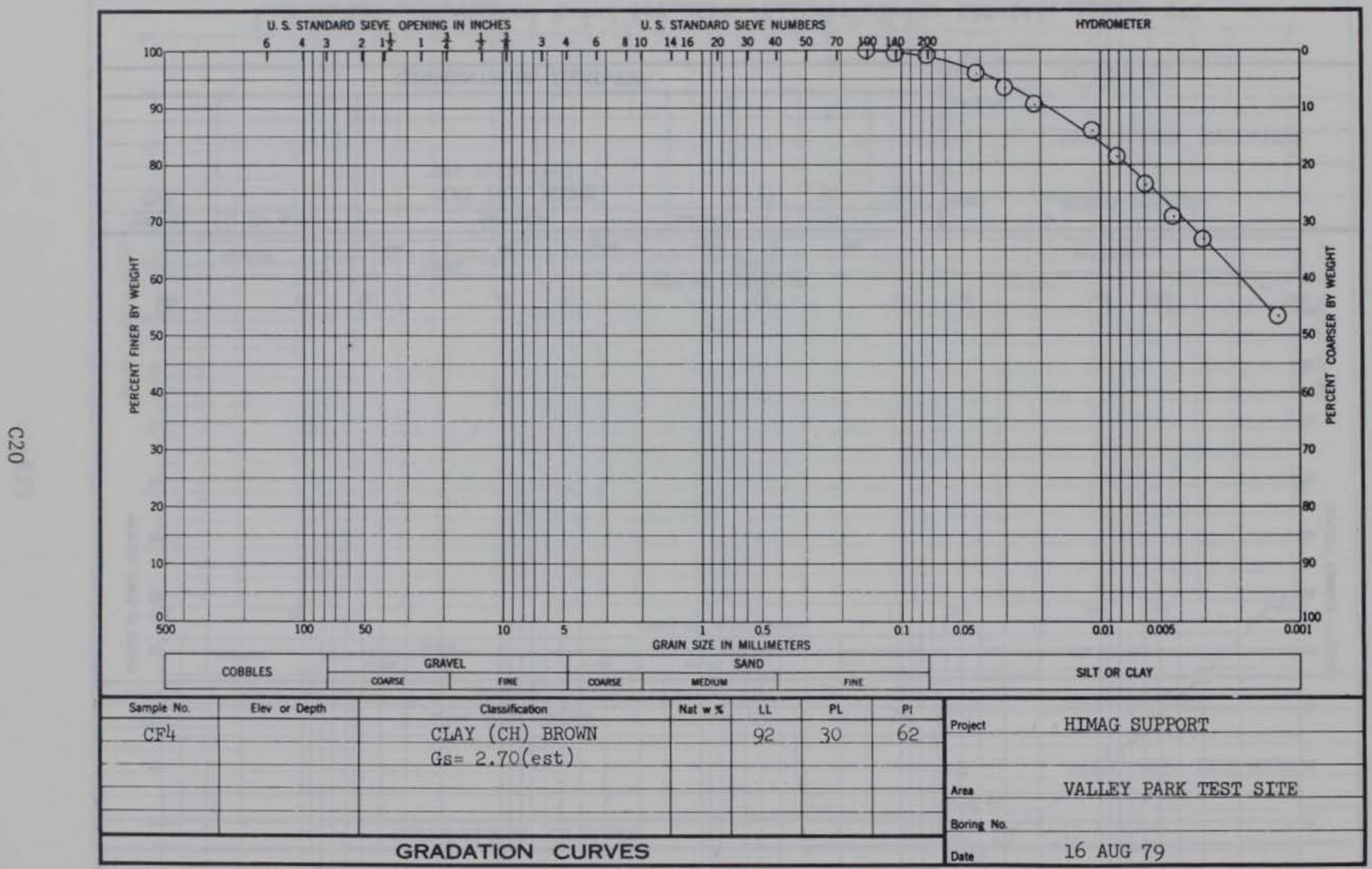

Figure C19. Gradation curve for site 7B, test series 139-144, sample CF4 


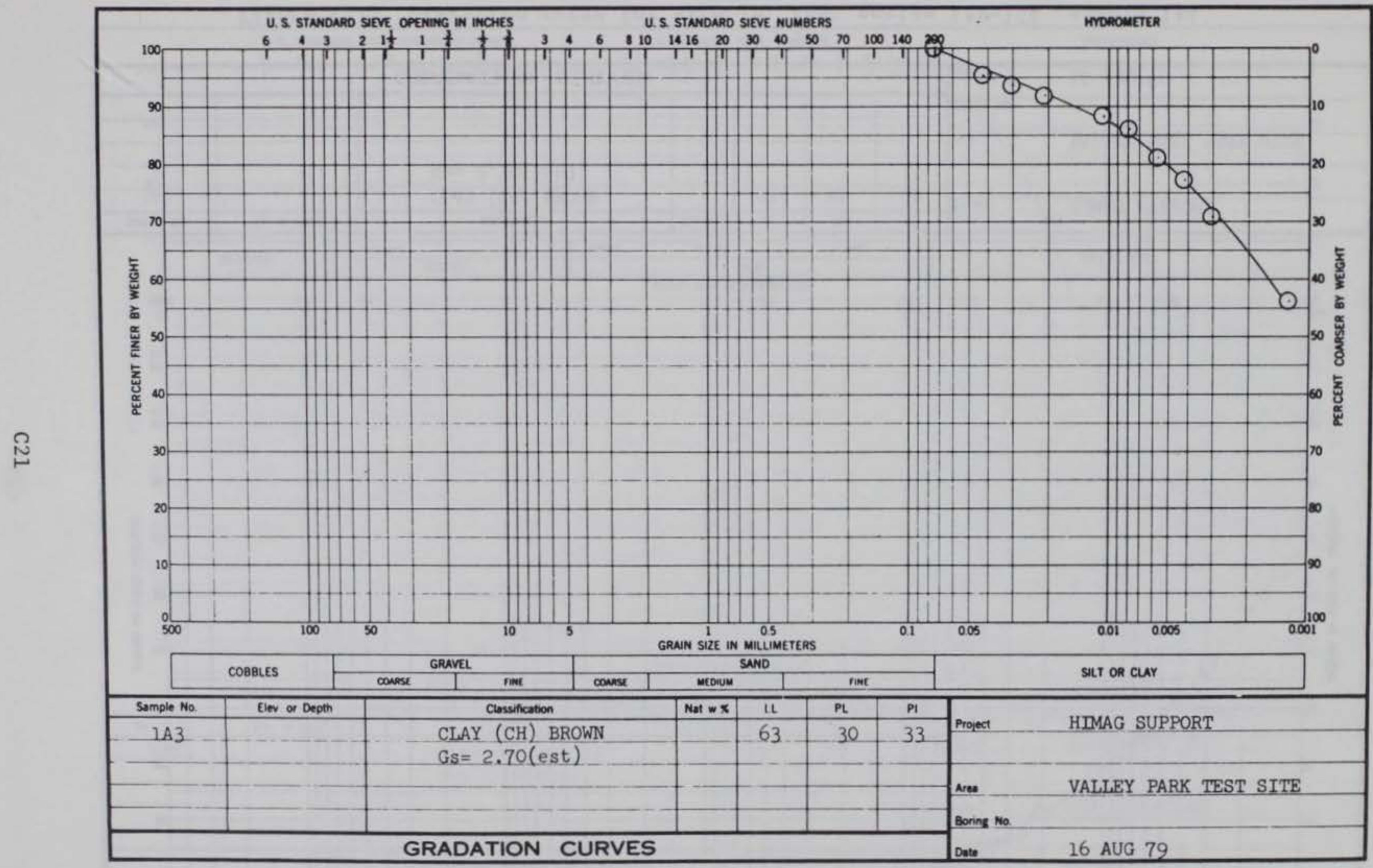

Figure C20. Gradation curve for site 7B, test series $139-144$, sample $1 \mathrm{~A} 3$ 


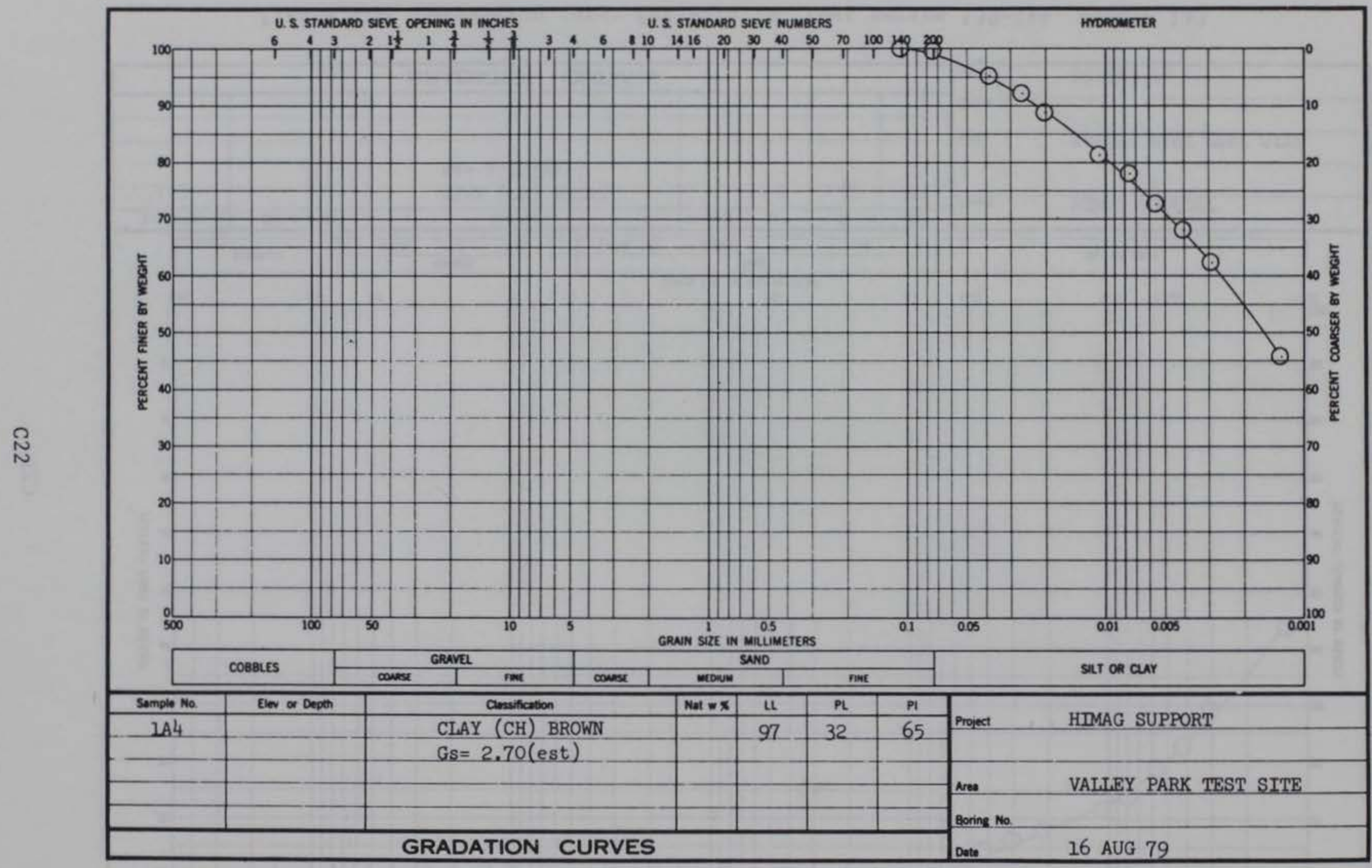

Figure C21. Gradation curve for site 7B, test series 139-144, sample 1A4 


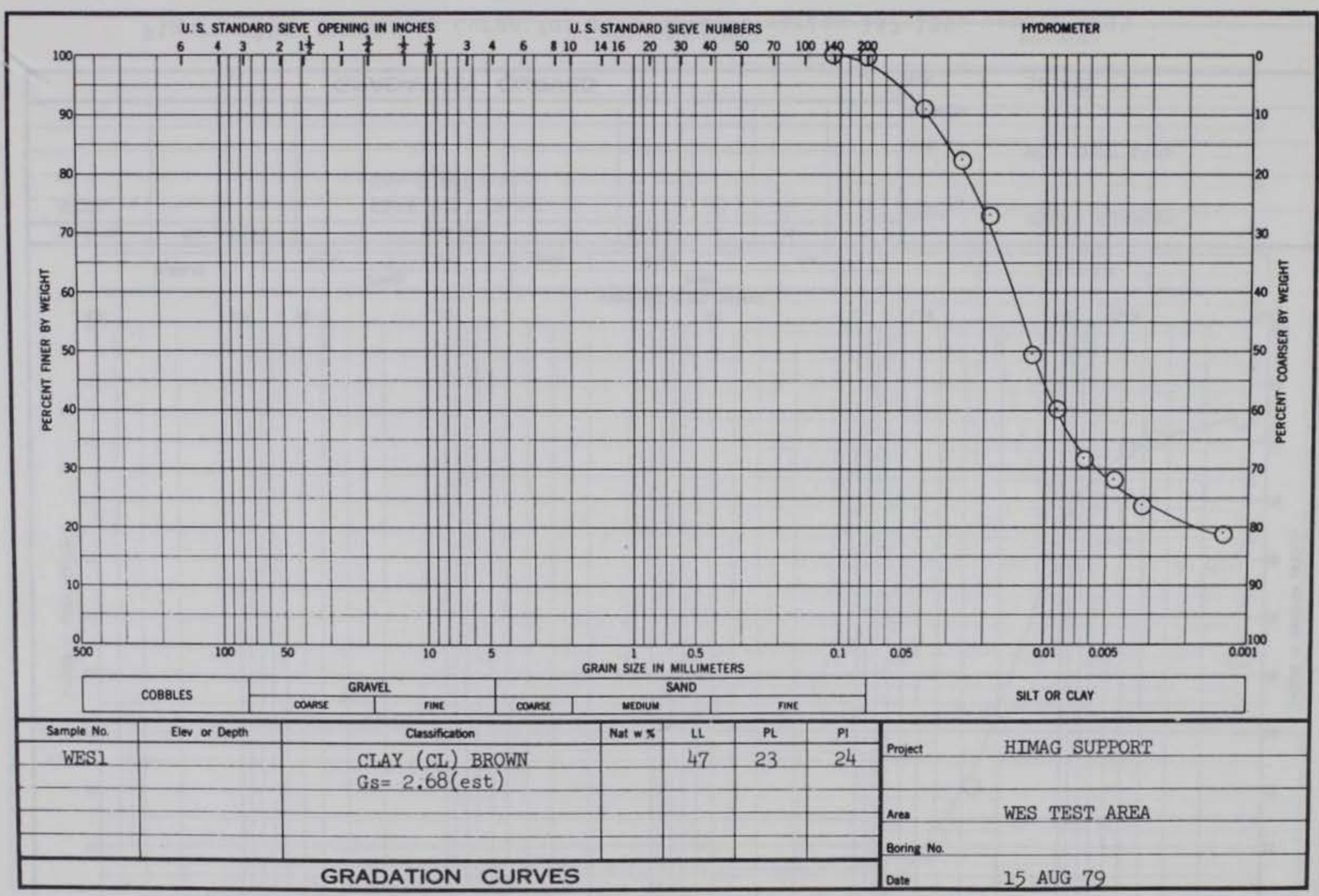

Figure C22. Gradation curve for site 8, test series 157-159, sample WES1 


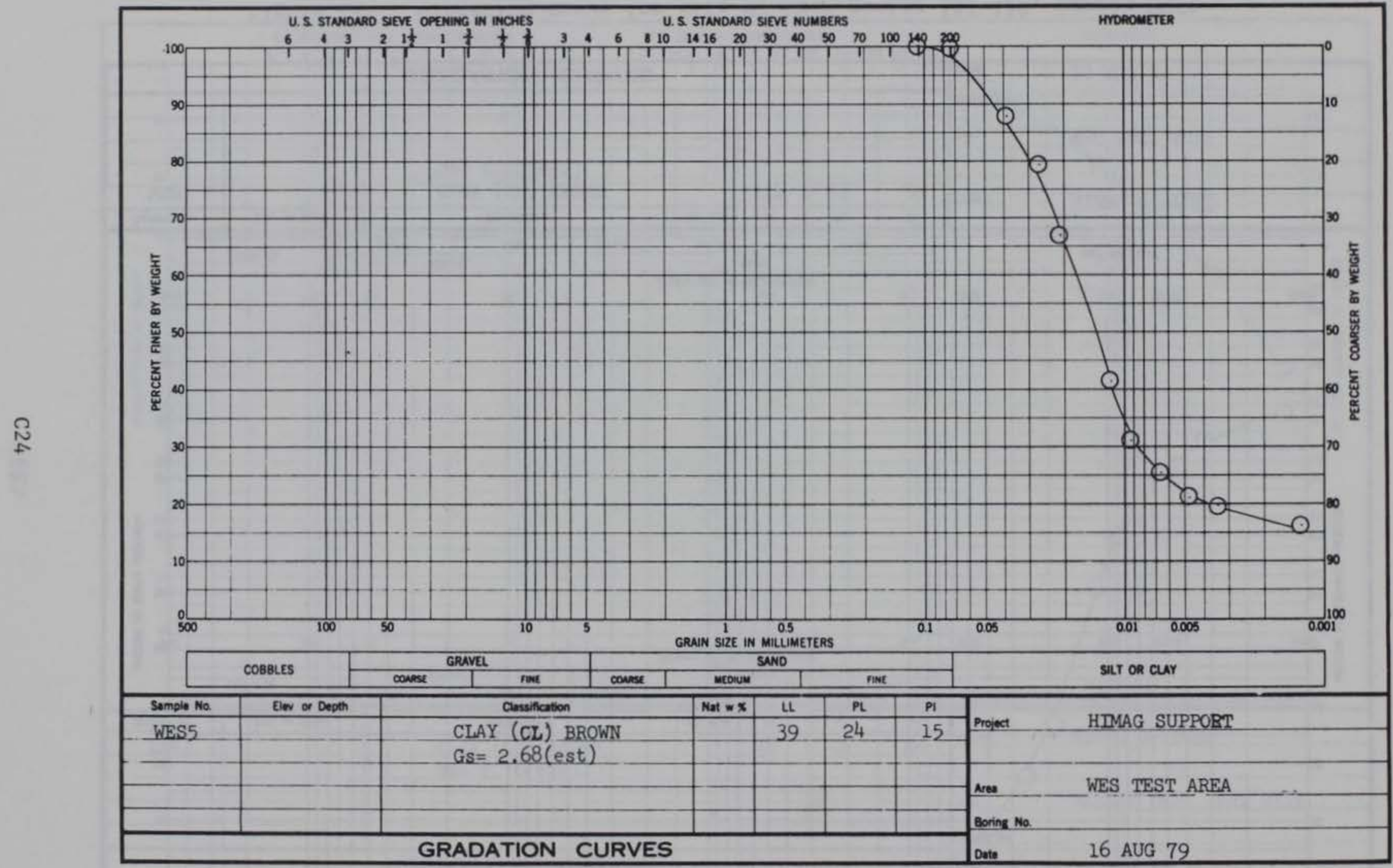

Figure C23. Gradation curve for site 8, test series 157-159, sample WES5 


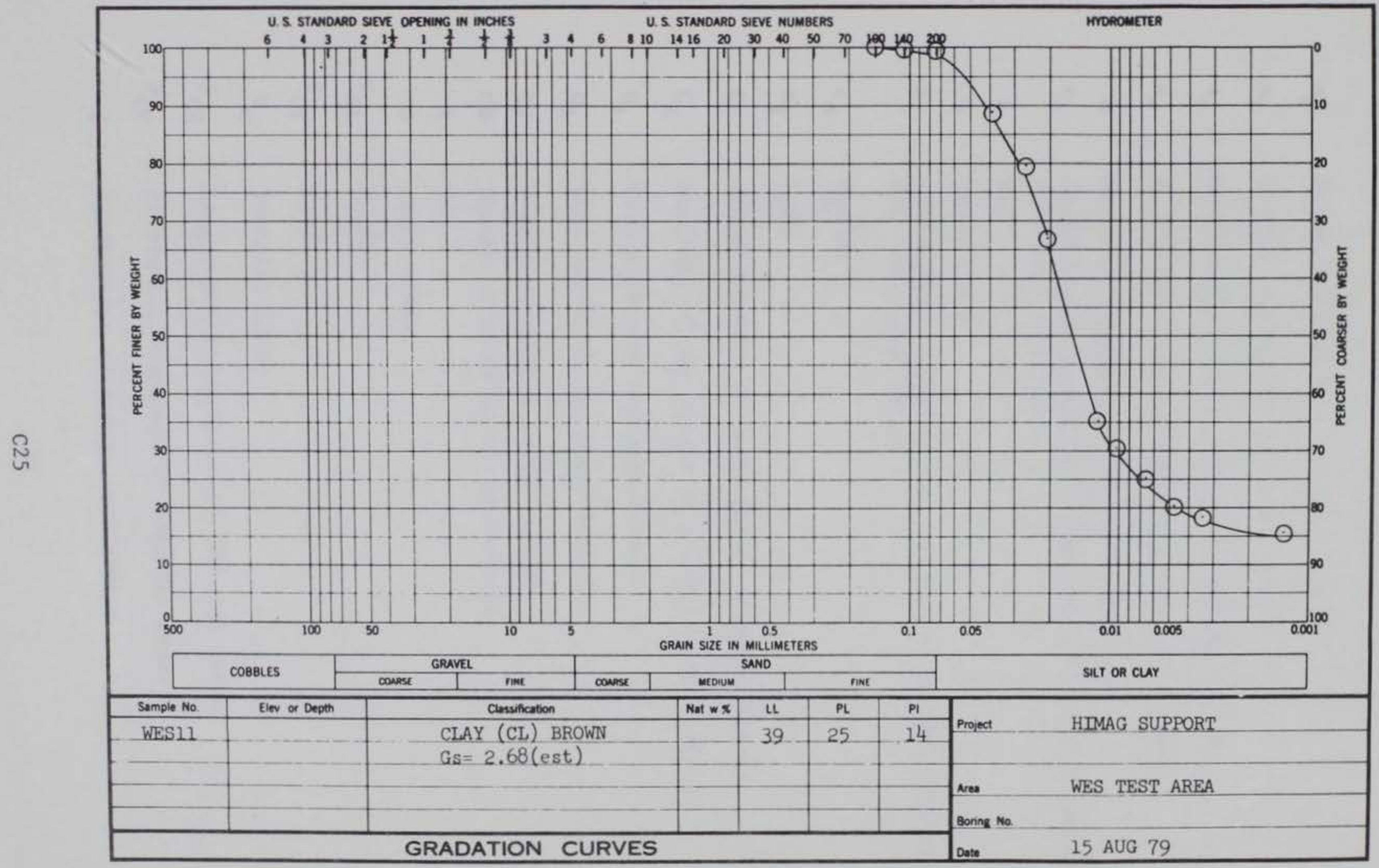

Figure C24. Gradation curve for site 8, test series 157-159, sample WES11 


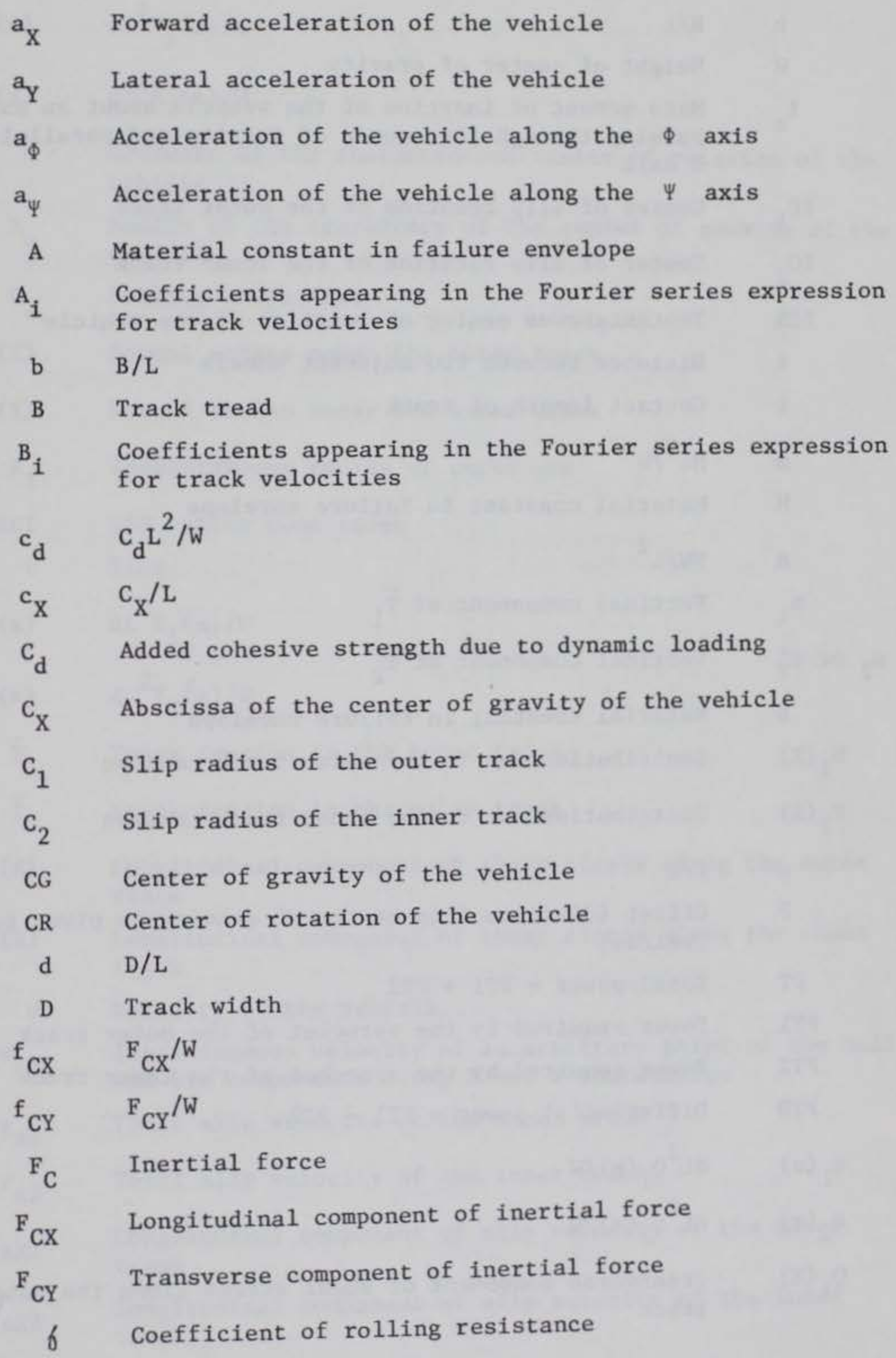




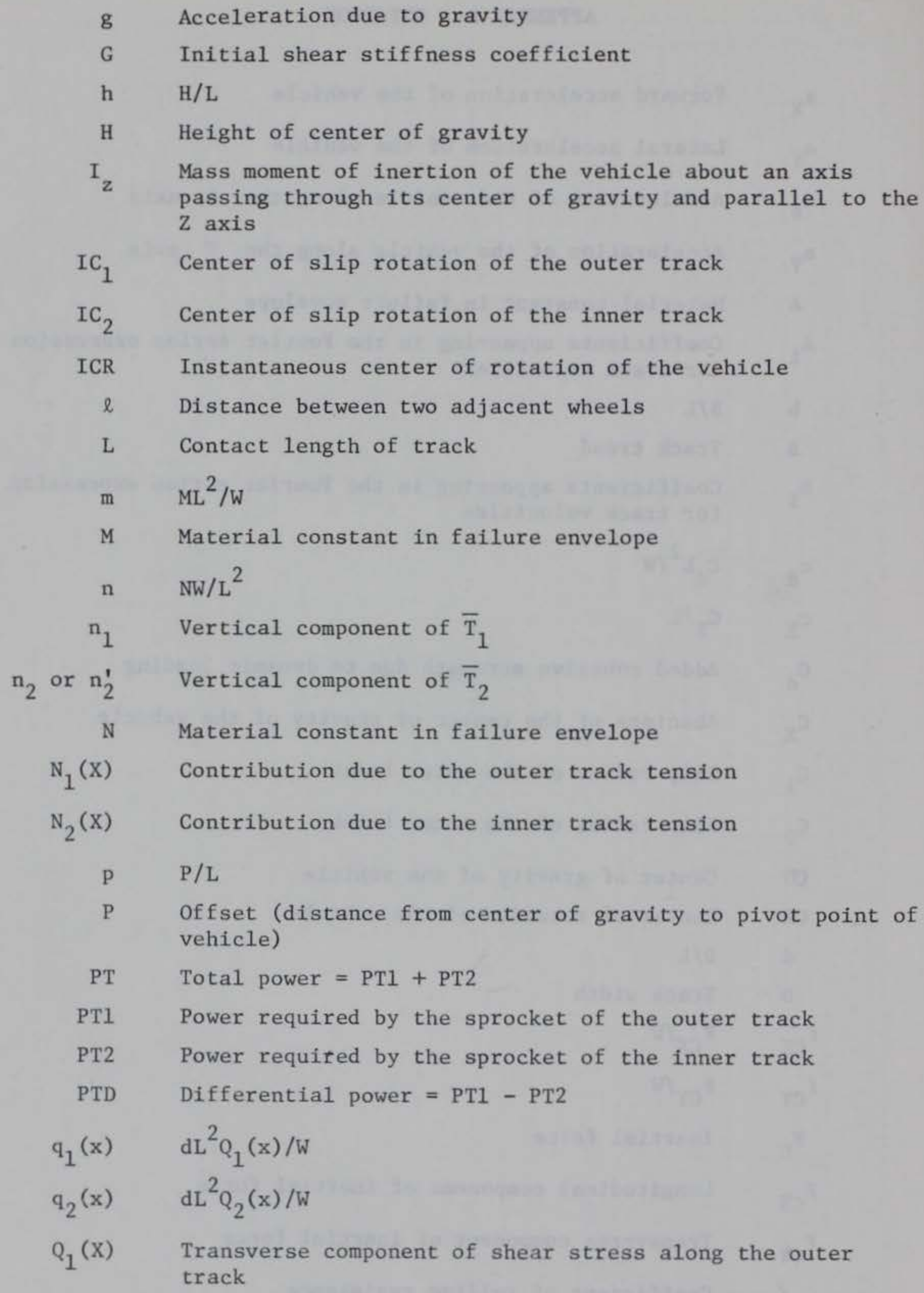




$$
\begin{aligned}
& \mathrm{Q}_{2}(\mathrm{X}) \quad \begin{array}{l}
\text { Transverse component of shear stress along the inner } \\
\text { track }
\end{array} \\
& r_{1}(x) \quad d^{2} R_{1}(x) / W \\
& r_{2}(x) \quad d^{2} R_{2}(x) / W \\
& \tilde{\mathrm{R}} \text { Ordinate of the instantaneous center of rotation of the } \\
& \text { vehicle } \\
& R_{0} \quad \text { Radius of the trajectory of the center of gravity of the } \\
& \text { vehicle } \\
& \mathrm{R}_{\mathrm{S}} \quad \text { Rolling resistance } \\
& \mathrm{R}_{1}(\mathrm{X}) \quad \text { Normal stress under the outer track } \\
& \mathrm{R}_{2}(\mathrm{X}) \quad \text { Normal stress under the inner track } \\
& \mathrm{R}_{\mathrm{I}} \quad \text { Instantaneous radius of curvature } \\
& \text { RCI WES rating cone index } \\
& \text { t Time } \\
& \mathrm{t}_{1}(\mathrm{x}) \quad \mathrm{dL}^{2} \mathrm{~T}_{1}(\mathrm{x}) / \mathrm{W} \\
& t_{2}(x) \quad d^{2} T_{2}(x) / W \\
& \overline{\mathrm{T}}_{1} \quad \text { Track tension in the inner track } \\
& \overline{\mathrm{T}}_{2} \quad \text { Track tension in the outer track } \\
& \mathrm{T}_{1}(\mathrm{X}) \quad \text { Longitudinal component of shear stress along the outer } \\
& \text { track } \\
& \mathrm{T}_{2}(\mathrm{X}) \quad \begin{array}{l}
\text { Longitudinal component of shear stress along the inner } \\
\text { track }
\end{array} \\
& \text { v Velocity of the vehicle }
\end{aligned}
$$

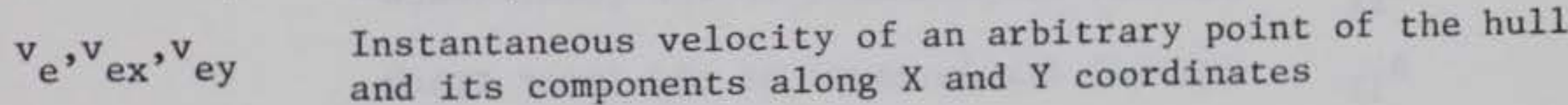

$$
\begin{aligned}
& v_{\text {s1 }} \quad \text { Total slip velocity of the outer track } \\
& v_{\text {s2 }} \quad \text { Total slip velocity of the inner track } \\
& v_{\text {sX1 }} \quad \text { Longitudinal component of slip velocity of the outer } \\
& \text { track } \\
& v_{s X 2} \quad \text { Longitudinal component of slip velocity of the inner } \\
& \text { track }
\end{aligned}
$$




\begin{tabular}{|c|c|}
\hline $\mathrm{v}_{\mathrm{sY} 1}$ & $\begin{array}{l}\text { Transverse component of slip velocity of the outer } \\
\text { track }\end{array}$ \\
\hline $\mathrm{v}_{\mathrm{sY} 2}$ & $\begin{array}{l}\text { Transverse component of slip velocity of the inner } \\
\text { track }\end{array}$ \\
\hline${ }^{v} \mathrm{X}$ & Longitudinal component of velocity of the vehicle \\
\hline $\mathrm{v}_{\mathrm{X} 1}$ & Longitudinal component of velocity of the outer track \\
\hline $\mathrm{v}_{\mathrm{X} 2}$ & Longitudinal component of velocity of the inner track \\
\hline $\mathrm{v}_{\mathrm{Y}}$ & Transverse component of velocity of the vehicle \\
\hline $\mathrm{v}_{\phi}$ & Component of velocity of the vehicle along the $\Phi$ axis \\
\hline $\mathrm{v}_{\psi}$ & Component of velocity of the vehicle along the $\psi$ axis \\
\hline $\mathrm{W}$ & Weight of the vehicle \\
\hline $\mathrm{W}_{\mathrm{N}}$ & Component of weight of the vehicle normal to the terrain \\
\hline $\mathrm{W}_{\mathrm{T}}$ & $\begin{array}{l}\text { Component of weight of the vehicle parallel to the } \\
\text { terrain }\end{array}$ \\
\hline$x$ & $\mathrm{X} / \mathrm{L}$ \\
\hline $\mathrm{X}, \mathrm{Y}, \mathrm{Z}$ & Local coordinate system \\
\hline y & $\mathrm{Y} / \mathrm{L}$ \\
\hline $\mathrm{z}$ & $\mathrm{Z} / \mathrm{L}$ \\
\hline$\alpha$ & Side-slip angle \\
\hline$B$ & $\ell / L$ \\
\hline 1 & Angle of slip direction of the outer track \\
\hline$\gamma_{2}$ & Angle of slip direction of the inner track \\
\hline$\Delta$ & Shearing deformation \\
\hline$\Delta 11$ & Initial displacement of the outer track \\
\hline$\Delta_{\text {I2 }}$ & Initial displacement of the inner track \\
\hline$\Delta_{1}$ & Shearing deformation of soil under the outer track \\
\hline$\dot{\Delta}_{1}$ & Time rate of shearing deformation \\
\hline$\Delta_{2}$ & Shearing deformation of soil under the inner track \\
\hline$\dot{\Delta}_{2}$ & Time rate of shearing deformation \\
\hline
\end{tabular}




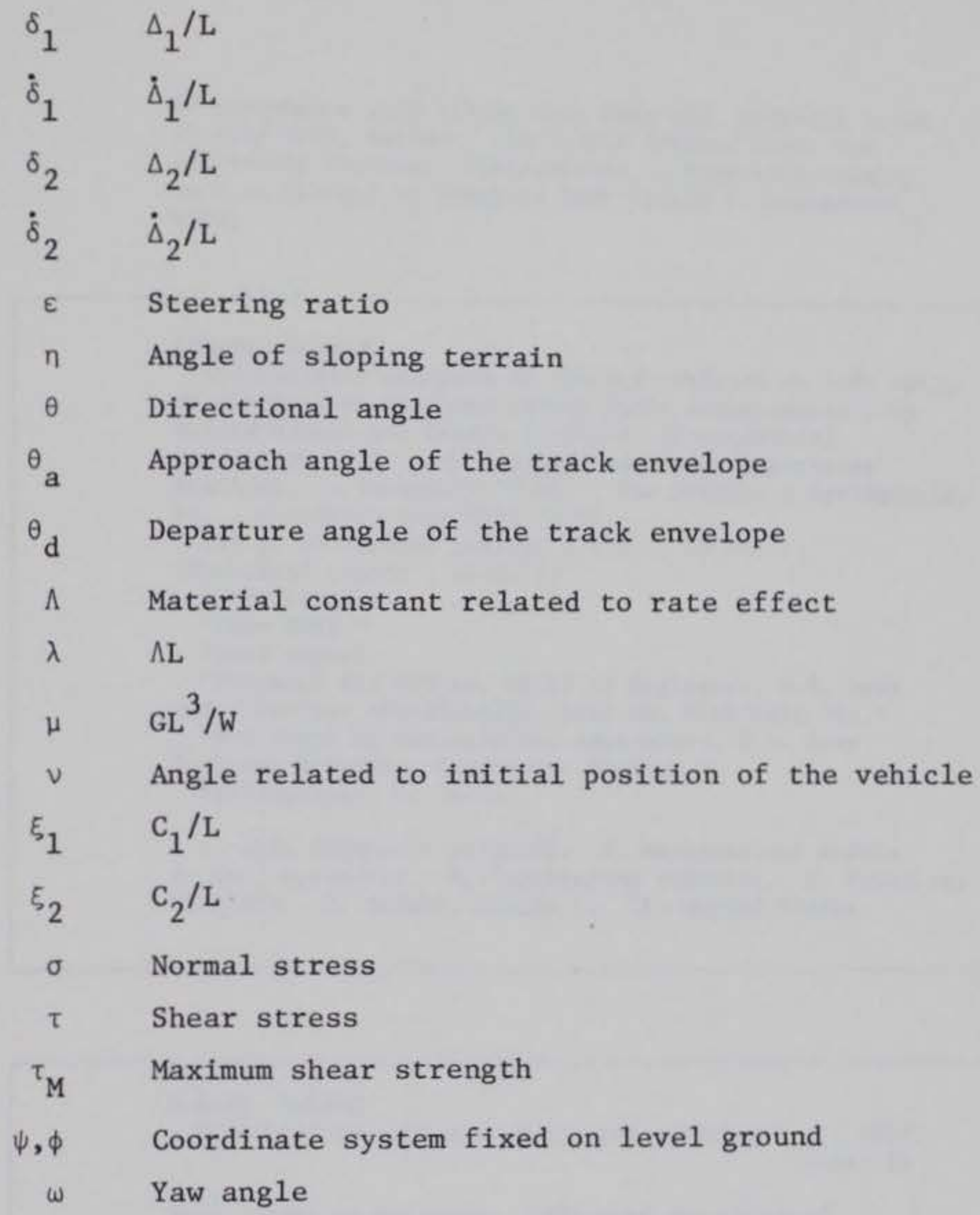


In accordance with letter from DAEN-RDC, DAEN-ASI dated 22 July 1977, Subject: Facsimile Catalog Cards for Laboratory Technical Publications, a facsimile catalog card in Library of Congress MARC format is reproduced below.

Rohani, Behzad

Steerability analysis of tracked vehicles on soft soil; theoretical predictions versus field measurements / by Behzad Rohani and George Y. Baladi (Geotechnical

Laboratory, U.S. Army Engineer Waterways Experiment Station). .- Vicksburg, Miss. : The Station ; Springfield, Va. : available from NTIS, 1982.

214 p. in various pagings ; i11. ; $27 \mathrm{~cm} .--$

(Technical report ; GL-82-4)

Cover title.

"June 1982."

Final report.

"Prepared for Office, Chief of Engineers, U.S. Army under Project 4A161102AT22, Task C0, Work Unit 001."

"Monitored by Geotechnical Laboratory, U.S. Army Engineer Waterways Experiment Station."

Bibliography: p. 20-21.

1. AGIL (Computer program), 2. Mathematical models. 3. Soil mechanics. 4. Tracklaying vehicles. 5. Vehicles, Military. I. Baladi, George Y. II. United States.

Rohani, Behzad

Steerability analysis of tracked vehicles : ... 1982.

(Card 2)

Army. Corps of Engineers. Office of the Chief of

Engineers. III. U.S. Army Engineer Waterways Experiment Station. Geotechnical Laboratory. IV. Title

V. Series: Technical report (U.S. Army Engineer Waterways Experiment Station); GL-82-4.

TA7.W34 no.GL-82-4 